\title{
Pronominal reference in sentences about persons or things investigated by temporal and spatial neuroimaging
}

Citation for published version (APA):

Hammer, A. (2005). Pronominal reference in sentences about persons or things investigated by temporal and spatial neuroimaging. [Doctoral Thesis, Maastricht University]. Datawyse / Universitaire Pers Maastricht. https://doi.org/10.26481/dis.20050630ah

Document status and date:

Published: 01/01/2005

DOI:

10.26481/dis.20050630ah

Document Version:

Publisher's PDF, also known as Version of record

\section{Please check the document version of this publication:}

- A submitted manuscript is the version of the article upon submission and before peer-review. There can be important differences between the submitted version and the official published version of record.

People interested in the research are advised to contact the author for the final version of the publication, or visit the DOI to the publisher's website.

- The final author version and the galley proof are versions of the publication after peer review.

- The final published version features the final layout of the paper including the volume, issue and page numbers.

Link to publication

\footnotetext{
General rights rights.

- You may freely distribute the URL identifying the publication in the public portal. please follow below link for the End User Agreement:

www.umlib.nl/taverne-license

Take down policy

If you believe that this document breaches copyright please contact us at:

repository@maastrichtuniversity.nl

providing details and we will investigate your claim.
}

Copyright and moral rights for the publications made accessible in the public portal are retained by the authors and/or other copyright owners and it is a condition of accessing publications that users recognise and abide by the legal requirements associated with these

- Users may download and print one copy of any publication from the public portal for the purpose of private study or research.

- You may not further distribute the material or use it for any profit-making activity or commercial gain

If the publication is distributed under the terms of Article $25 \mathrm{fa}$ of the Dutch Copyright Act, indicated by the "Taverne" license above, 


\section{Pronominal Reference in SENTENCES ABOUT PERsons OR ThINGS}

investigated by temporal and spatial neuroimaging 
Illustration: Merseburger Zaubersprüche (Cod. Hs. 136, fol.85 r), Domstiftsbibjiothek Merseburg. Reprinted with permission.

Printed by Datawyse, Maastricht

Copyright 2005 by Anke Hammer. All rights reserved. 


\section{Pronominal Reference in SENTENCES ABout PERsons or Things}

investigated by temporal and spatial neuroimaging

\section{PROEFSCHRIFT}

ter verkrijging van de graad van doctor aan de Universiteit Maastricht, op gezag van de Rector Magnificus,

Prof. mr. G.P.M.F. Mols

volgens het besluit van het College van Decanen,

in het openbaar te verdedigen

op donderdag 30 juni 2005 om 12.00 uur door

Anke Hammer

geboren op 8 oktober 1976 te Burg, Duitsland

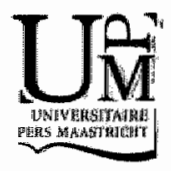




\section{Promotor:}

Prof. dr. R.W. Goebel

\section{Copromotor:}

Dr. B.M. Jansma

\section{Beoordelingscommissie:}

Prof. dr. d. van Heerden

Dr. J. van Berkum

Dr. L. Blomert

Prof. dr. P. Hagoort

Prof. dr. N.O. Schiller

\section{(Voorzitter)}

(Universiteit van Amsterdam)

(F.C. Donders Center / Katholieke Universiteit / Max Planck Institute for Psycholinguistics; Nijmegen)

(NWe research reported in this thesis was supported by a grant of the Dutch Science Foundation (NWO) and by a grant of the German Science Foundation (DFG). 
Meiner Familie 


\section{Merseburger Zaubersprüche}

\section{Zeflengerteue Abschrift}

Eiris sacun id ist sazom hera diuoder suma hapt heptidun sumaherilezidum sumaclu bodun umbicuonito utidi insprinc hapt bandun inuar uigandun. $\mathrm{H}$.

Phol ende wuodar tuorun zitholza duvuant denobilderes wolon sin unoz birenkict thubiguolen sinhthunt swnina era suister thubiguolen fria wolla ema suster thu biguollen uuodan so he urola conda sosebentronki sosebluotrenki soselidi renki ben zibena bluot zibluoda. lid zigelliden sosegelimida sin

\section{Ubersetzung (von Wolfgang Beck)}

Einst saben Idisi, saßen aluf den Kriegerscharen. Einige fesselten einen Gefangenen, einige hemmten die Heere, einge zertrennten scharfe Fesseln. Entspringe den Fesseln, entfahre den Feinden! $\mathbb{H}$.

Phol und Wodan begaben sich in den Wald. Dort wurde dem Fohlen Balderss der der FuB eingerenkt. Da besangen ihn Sinhtgunt and Sunma, ihre Schwester. Da besangen ihn Frija und Volla, ihre Schwester. Da besang ihn Wodan, so wie er es gut verstand: Wenn Knochemrenkung, wenn Blutrenkung, wenn Gelenkrenkung: Knochen zu Knochen, Blut zu Blut, Glied zu Glied! So seien sie zusammengefügt!

Im 10. Jahnhmdert schrieb ein Monch die Merseburger Zauberspriche in althochdeutscher Sprache wahrscheinlich aus dem 8. Jahthundert auf das leere Vorsatzblatt einer Messhandschrift. Diese Zeilen wurden erst 1841 von Georg. Waitz entdeckt. Er verzichtete auf eine Veröffentlichung und überließ sie dem Gebrüdern Grimn zuir Bearbeitung. Jacob Grimm walle diese dann zum Thema seines Antrittsvortrag vor der Berliner Akademie am 3. Februar 1842. Die Merseburger Zaubersprüche erhiethen ihren Namen nach dem Fundort. Sie werden als Handschrift (Hs) 136 in der Bibliothelk des Domstifts Merseburg verwahrt. 


\section{Contents}

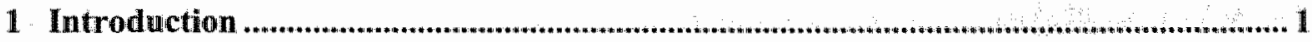

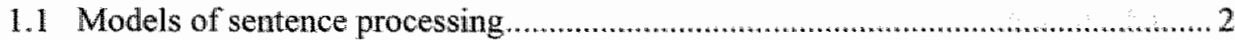

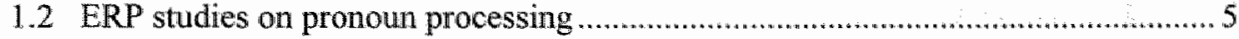

1.3 Anatomical locations of syntactic and semantic processing $\ldots \ldots \ldots \ldots \ldots \ldots \ldots \ldots \ldots \ldots \ldots . . . . . . . . \ldots$

1.4 Verbal Working memory and pronoun processing ............................................. 10

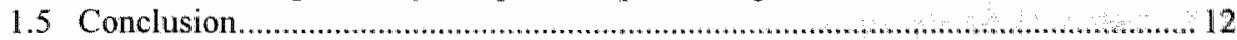

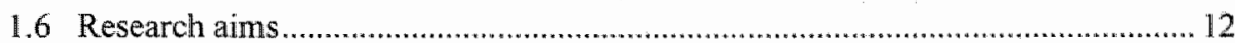

2 Promominal reference in sentences about persons or things: An electrophysiological

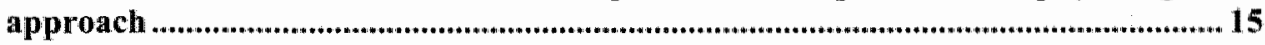

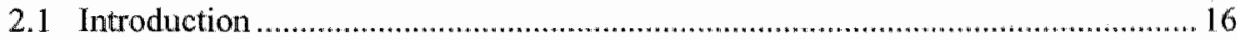

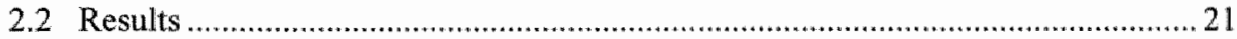

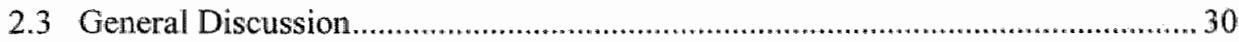

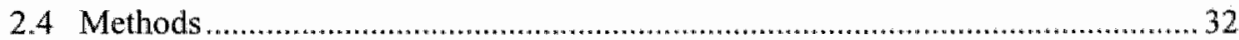

3 Pronoun resolution in sentences about persons or things investigated by Magnetoencephalography..................................................................................................... 37

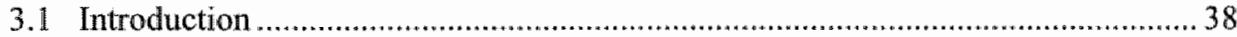

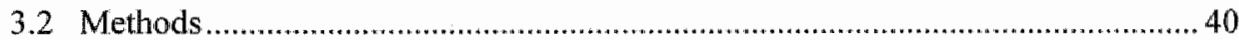

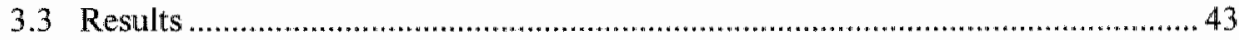

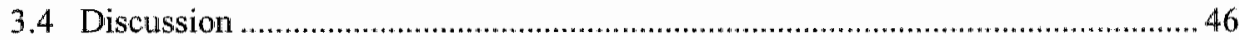

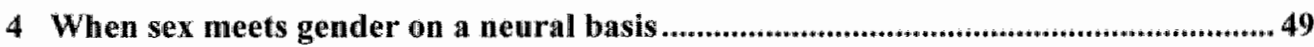

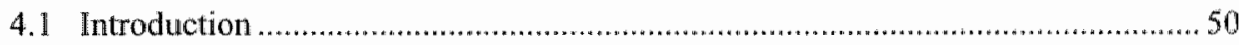

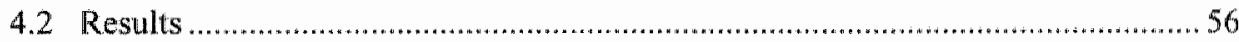

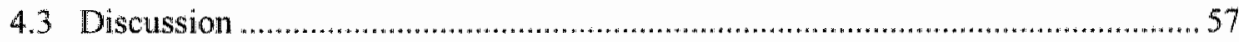

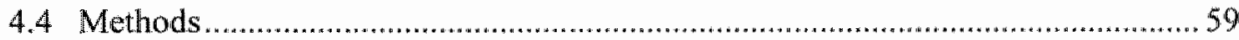

5 Interaction of semantic and syntactic information and working memory during

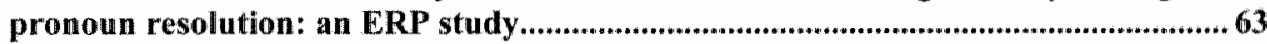

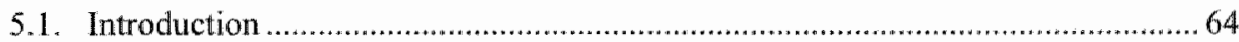

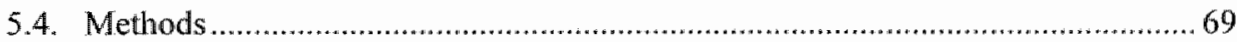

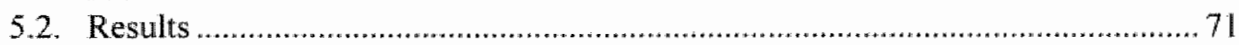

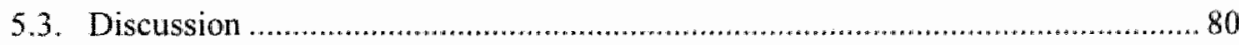


6 Interaction of semantic and syntactic gender information and working memory during pronoun resolution: an TMRI study

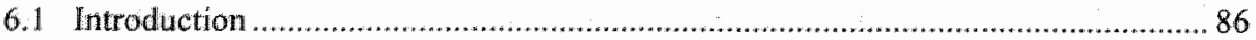

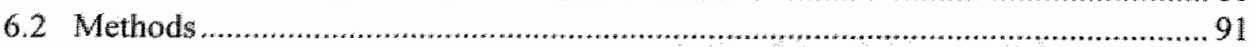

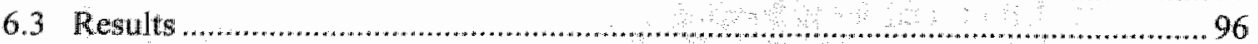

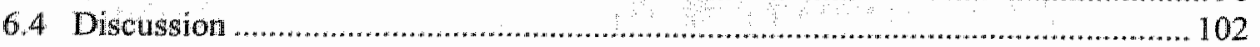

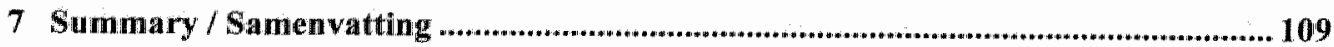

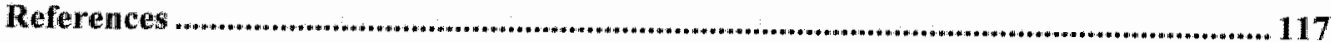

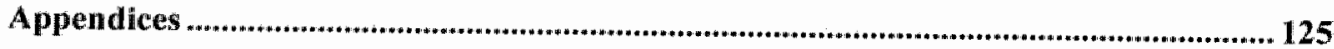

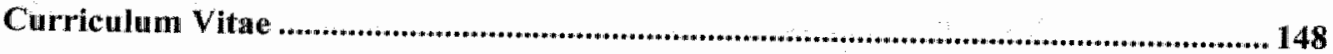


Reading is onc of our daily businesses. We read books for pleasure, newspapers for information, emails for communication and so on. Among many things, in order to understand a text, we have to integrate the meaning and the syntactic relations among words. Usually we are not aware of the details of this cognitive task because it goes so well, mostly automatic, especially when there is coherence in terms of syntactic and semantic information. However, what makes a text coherent?

Text coherence can be studied in many ways. One would be, and this is the focus of this thesis, to consider the way personal pronouns are processed. Pronouns are linguistic devices that allow a speaker to refer to an earlier mentioned person or thing. Consider the sentence: "The woman is happy because she fell in love." It is easy to tell that "she" refers back to the earlier mentioned woman. This phenomenon, where one item in a sentence refers back to an element mentioned earlier in the sentences, is also labeled as establishing co-reference, as in the case of pronouns. This process requires working memory demands on the comprehending system because earlier mentioned items have to be reactivated during a possible searching process for an appropriate antecedent. However, in order to establish one candidate as the target reference, pronouns must be matched to the antecedent conceptually (semantically) and syntactically (gender). In the German language, a woman ('die Frau') for example has a female biological gender with a corresponding feminine syntactic gender. However, a jacket ("die Jacke") has just a feminine syntactic gender with no semantic reflex.

During processing a sentence with an anaphoric expression the reader has to go through at least three different steps: (1) a conceptual/semantic match between pronoun and its antecedent, (2) a syntactic match between pronoun and antecedent in terms of number/gender and/or case marking, and (3) an activation of the discourse representation in working memory in order to make the connection between the pronoun and its antecedent, which occurred earlier in time in discourse.

\subsection{Models of sentence processing}

There are two main classes of models on sentence processing to be differentiated. They agree that different sub-processes underlie the comprehension process. However, they differ in their characterization of the processes involved and the time course of the use of certain sorts of information (Hagoort, Brown \& Osterhout, 1999; Frauenfelder \& Tyler, 1987). One class of these models are so called serial or syntax-first models (i.e. Frazier \& Fodor, 1987; Ferreira \& 
Clifton, 1988), which claim that the parser initially builds up a syntactic structure independent of semantic information. Within these models the syntactic component is explicitly restricted to the domain of syntactic information. Non-syntactic information does not influence the analysis of the sentence at that early stage. Semantic and pragmatic information are represented in a different component and are used at a later stage. Thus, these models assume that syntactic and semantic processes are supported by different components within the comprehension system, and these may be implemented by distinct brain regions at the neuronal level. The second class of models, so called interactive models, assume that syntactic and semantic information interact at any time during parsing (e.g. Marslen-Wilson \& Tyler, 1980; Trueswell \& Tanenhaus, 1994). In these models, all (available) sources immediately affect the parsing process without appealing a separate level of syntactic representation. Syntactic, lexical, and semantic/conceptual information are used interactively and continuously. For a recent debate concerning these controversial assumptions on a neural base, see Friederici (2002) versus Hagoort (2003).

\subsubsection{A psycholinguistic model of pronoun processing}

A major interest of psycholinguistic research is to find out how words, for example a pronoun and its potential antecedent are combined to form a coherent sentence. The pronoun has to be linked to a possible antecedent in terms of syntax and/or semantics - and this process is called co-reference. Garrod and Sanford (1994) presented a model with two stages for pronoun interpretation (see also Garrod \& Terras (2000) for a recent discussion about discourse role resolution) with (1) a low level automatic process associated with establisthing a link between anaphor and a previous expression (referred to as bonding), and (2) a stage, which is testing and resolving this link (referred to as integration). The bonding process could rely on a syntactic feature, i.e. the syntactic gender agreement of pronoun and antecedent. The integration process is responsible for evaluating the established link, i.e. by weighting syntactic and semantic information. Garrod and Sanford pointed out that the integration of the pronoun would come about through an interaction between the syntactic (gender) and semantic information of the pronoun and the focus state of the prior discourse representation. The term "focus" means that certain elements are more prominent in a discourse than others. If this is the case, the comprehension system makes an early commitment to the full interpretation of the pronoun. When either one of these conditions does not hold, commitment is delayed untill further information has been encountered. In other words, during a first step the comprehension system loosely links the pronoun to a potential antecedent by a fast and 
automatic process (bonding). This link is evaluated a little later in order to avoid errors in comprehension. If this evaluation process (resolution) reveals that the bonding was right in terms of semantic and syntactic information, the comprehension system confirms the establishment of co-reference between pronoun and antecedent. The evaluation process may be speeded up by the congruency of three information types: semantic, syntactic and focus information. This model is substantiated by the following reading experiment: Garrod, Freudenthal, and Boyle (1994) presented sentences containing contextually congruent or incongruent verbs while eye-movements were monitored in order to examine how early the inconsistency would be detected. Example material would be:

\footnotetext{
Dangerous Incident in the Pool

Elizabeth (1)/Allexander (2) was an inexperienced swimmer and wouldn't have gone in if the male lifeguard (3) hadn't been standing by the pool. But as soon as she/he got our of her/his depth she he started to panic and wave her/his hands about in a frenzy.

(I) Within seconds she (1) sank/*jumped into the pool.

(II) Within seconds he (2 or 3) sank/fumped into the pool.
}

The authors observed elongated first pass fixations for incongruent compared to congruent verbs. This incongruence effect was found within the first $100 \mathrm{~ms}$ on the verb. However, it only occurred when the sentences contained pronouns that referred unambiguously to the focused subject of the sentence (as in I). Garrod and colleagues did not test what would happen if the bonding process were interrupted by an explicit incongruence of pronoun and antecedent:

Garnham, Oakhill, Ehrlich, and Carreiras (1995, experiment 1) tested whether a mere syntactic cue could affect the interpretation of a pronoun. The authors compared the effects of gender cueing in Spanish and in French about persons (biological/conceptual gender) and about things (syntactic gender) by measuring reading times and comprehension. An example sentence for persons that involves biological gender cueing was 'Rosaline arrested Paul because he was stealing a car.' In this sentence type, the pronoun can be referred to one of the persons by its biological gender alone because the two persons differ in gender. In contrast, in the sentence 'Peter arrested Paul because he was stealing a car.' the pronoun integration requires more inferential processing because it cannot be solved by gender information alone. Similar sentences were also created for things, which by necessity involve syntactic gender cueing - but no biological gender cueing. Garnham et al. (1995) did observe two gendercueing effects: (1) Reading times were shorter in the cued condition compared to the non- 
eued conditions for things and persons. While this gender-cueing effect was attributed to 'superficial' syntactic encoding of pronouns in sentences referring to things, the authors viewed it as being either superficial or semantically driven in the sentences about persons. (2) Interestingly, a stronger gender-cuing effect for sentences about persons compared to things was found for question answering times after sentence endings. The authors interpreted this effect as a change in information processing over time: to establish co-reference the semantic information (biological gender cue) becomes more relevant after sentence completion than the syntactic information (syntactic gender cue). This means that besides the question about what kind of information (syntactic and/or semantic) is accessed during pronoun integration there is the question about the time-course of establishing the co-reference during discourse comprehension using different types of information.

Other psycholinguistic studies also have shown the involvement of conceptual/semantic and syntactic information to establish the link between a possible antecedent and a pronoun (Rigalleau \& Caplan, 2000). Others suggested that it is either conceptual/semantic information (Gernsbacher, 1991), or syntactic information, i.e. more surface related processing (Gordon \& Hendrick, 1998) for establishing the co-reference.

Taken together, behavioral data showed a complex pattern that is not yet consistent. One reason for the variety of findings could be that eye-movements and reaction times have a limited temporal resolution concerning on-line measuring of cognitive processes. The model of pronoun processing proposed by Garrod and Sanford (1994) belongs to the class of syntax first model. Methods with higher temporal resolution may be better suited to validate or reject the given assumptions on pronoun resolution.

\section{2 ERP studies on pronoun processing}

Event-related Potentials (ERPs) are minute voltage fluctuations of electrical activity produced by neurons in the brain that are recorded from various points on the scalp during the processing of cognitive tasks (see Münte, Urbach, Dïzel \& Kutas, 2000; Rugg \& Coles, 1995; for language see Kutas, Federmeier, Coulson, King \& Münte, 2000).

Previous ERP-studies have led to the identification of several components involved in semantic and syntactic processing. In a series of pioneering studies, Kutas and Hillyard ( 1980 , 1983) found that semantic anomalies elicit a large negative component with a peak around $400 \mathrm{~ms}$ with a right centro-parietal maximum subsequent to the onset of the anomalous word, 
the so-called N400 component. This $\mathrm{N} 400$ has been shown to be sensitive to semantic integration (Kutas \& van Petten, 1994; Kutas \& King, 1995), as well as to discourse integration (St. George, Kutas, Martinez \& Sereno, 1999; Van Berkum, Hagoort \& Brown, 1999; Frisch \& Schlesewsky, 2001). Another important finding is that the amplitude and the latency of the N400 component depend on word-class. There are two word-classes to differentiate: open elass or content words (such as nouns, verbs, adjectives or most adverbs) and closed class or function words (such as pronouns, determiners, conjunctions or prepositions). For example, Münte, Wierenga, Weyerts, Szentkuti, Matzke, and Johannes (2001, first experiment) compared these two word-classes with a word-list experiment. In this experiment, the N400 component was considerably smaller for closed-class words. This finding is in line with earlier studies (Kutas \& Hillyard, 1983; Van Petten \& Kutas, 1991; for a review see Kutas \& van Petten, 1994). With respect to the processing of syntactic information an early left anterior negativity (LAN) and a late centro-parietal positivity (P600/SPS, syntactic positive shift) have been identified. The LAN has been observed to follow syntactic violations such as phrase structure errors (Friederici, Steinhauer, Mecklinger \& Meyer, 1998; Münte, Heinze \& Mangun, 1993; Neville, Nicol, Barss, Forster \& Garret, 1991 ) and working memory demands (Münte, Schiltz \& Kutas, 1998b; Streb, Rösler \& Hennighausen, 1999). The $\mathrm{P} 600$ has been observed in response to syntactic violations (Osterhout, Holcomb \& Swinney, 1994; Osterhout \& Holcomb, 1992; Friederici, Steinhauer \& Frisch, 1999; for a review see Hagoort, Brown \& Osterhout, 1999) or, more generally, in situations requiring the reanalysis of the sentence (Osterhout et al., 1994; Friederici and Mecklinger, 1996: Münte, Heinze, Matzke, Wierenga \& Johannes, 1998a). The P600 typically has a peak-latency around $500-600 \mathrm{~ms}$ after the critical word and a duration of several hundred milliseconds (Osterhout \& Holcomb, 1992; Hagoort et al., 1999).

\subsection{Symactic co-reference}

Osterhout and Mobley (1995) focused on the processing of violations in a sentence. In a first experiment requiring the judgment of sentence acceptability two types of violations were presented.

\footnotetext{
The hungry guest helped themselves/himself to the food." (Reflexile-antecedent number violation),

'The successful woman congratulated herself/himself on the promotion.'

(Reflexive-antecedent gender violation).
} 
The authors stated that if violations in these two cases were purely syntactic, a $\mathrm{P} 600$ should be expected. In contrast, if the sentences involve semantic violation, an N400 was expected. Violations of both number and gender of the reflexive pronoun elicited a P600-like component, but no $\mathrm{N} 400$. This result was interpreted as indicating a syntactic integration of the reflexive pronoun without a semantic reflex. Furthermore, the experiment revealed exact information about the time course of establishing co-reference. Following Osterhout and Mobley the process starts at about $500 \mathrm{~ms}$ after onset of the reflexive pronoun.

\subsubsection{Semantic co-reference}

King and Kutas (1997, see also Kutas et al., 2000) examined the response to pronouns that refer to a stereotypically male or female antecedent. The subject noun in the experimental sentence was an occupational title that was more or less likely to be filled by a person of female or male gender, i.e. "The engineer redesigned the circuit because he/she had detected a flaw.' Readers appear to treat the female pronoun as mismatching because the probability of female engineers is small in reality. The ERPs elicited by such a non-stereotypical pronouns showed a large anterior negativity after about $200 \mathrm{~ms}$ and was related by the authors to working memory and/or discourse analyses. Alternatively, however, this effect could be interpreted as result of a syntactic violation. The 'engineer' was analyzed as masculine and as soon as the system processes the pronoun 'she', it discovers a syntactic mismatch. Another finding in this study was that mismatching pronouns elicited a larger N400 component. This effect could indicate an involwement of semantic and/or discourse processing of words during the integration of the pronoun into the sentence. However, please note that in a related study by Osterhout, Bersick, and McLaughlin (1997) reflexive pronouns that mismatched the gender of occupational stereotypes elicited a P600 component, indicating no N400 related semantic processes. One possible explanation for the difference in results between these two studies is that personal pronouns and reflexive pronouns are treated differently by the language system.

\subsubsection{Syntactic and semantic co-reference:}

In a recent study Schmitt, Lamers, and Münte (2002) examined the separate effects of biological and syntactic gender violation during pronoun processing. The study was conducted in German because the German language differentiates between biological gender (FEMALE/MALE) and syntactic gender (feminine, masculine, neuter), especially in the cases 
of diminutives (das Bubchendaifineuler [the little boy]). Subjects read sentences in which an antecedent (BubchenMALeneuter [the little boy] or BubMaLemasculine) was introduced. Later a personal pronoun wass presented which either agreed with both gender types, agreed with the antecedent in terms of syntactic gender only, or in terms of biological gender only, or violated both agreements. Example materials would be:

\section{Diminutive as antecedlent:}

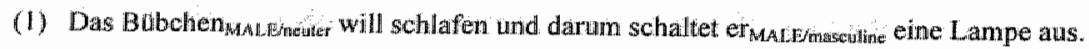
(biological gender agreement, syntaltic gender violation)

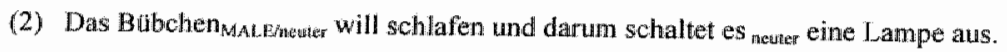
(biological gender violation, syntactic gender agreement)

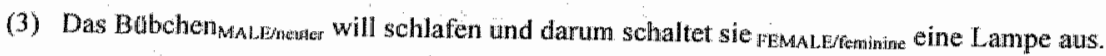
(biological gender wiolation, syntactic gender violation)

Non-Diminutive as antecedent:

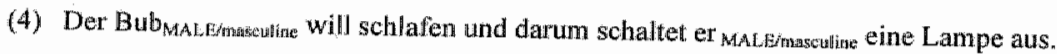
(biological gender agreement, syntactic gender agreement)

(5) Der Bub MALEinasculine will schliafen und darum schattet es (biological gender violation, syntactic gender violation)

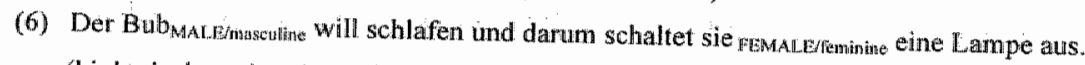
(biological gender violation syntactic gender violation)

(Translation: The (little) boy wants to sleep and therefore he/she/it switches the light off.)

Overall, the results showed salient P600 effects for pronouns, with violations being more positive than non-violations. This indicated that the establishment of reference involves syntactic reanalysis. Furthermore, the authors observed N400 effects in sentences with nondiminutives (violations being more negative than non-violations), but not with diminutives. This showed that conceptual/semantic integration is involved during non-diminutive but not during diminutive pronoun processing; at least it could not be violated.

\subsection{Anatomical locations of syntactic and semantic processing}

At present, there are no MEG and AMRI studies reported, which investigated the process of building up co-reference during pronoun resolution. Therefore, we should concentrate on anatomical locations of syntactic and semantic processing during other language processes. Traditionally "Broca's and Wernicke's area have been considered based on specific impairments of patients with focal brain damage to be the most relevant brain structures for 
language processing. This model has been modified and extended as a result of more precise neuroanatomical data and results from functional imaging studies (review: Brown, Hagbort \& Kutas, 2000; Martin \& Chao, 2001). In a PET study Indefrey, Hagoort, Herzog, Seitz, and Brown (2001) examined the cerebral blood flow (CBF) during syntactic processing in an experiment requiring the detection of grammatical errors in meaningless sentences. These sentences were compared with a control condition in which subjects had to read out unstructured strings of pseudo-words (no meaning) and function words. Indefrey and colleagues reported that an area of the left dorsolateral prefrontal cortex, adjacent to Broca's area activated by syntactic error detection, is involved in syntactic processing aspects. Whereas other prefrontal areas, i.e. right inferior frontal sulcus, and the left middle frontal gyrus, subserve general error detection processes. As an implication of their results on pseudo-word material the authors clarified the role of the left posterior prefrontal cortex in sentence-level processing, by showing that it is indeed syntax-related processing rather than a syntactically mediated semantic integration of word meanings that activates this cortical region.

A recent fMRI-study investigated the neuroanatomical correlates of semantic and syntactic information processing and possible interdependencies (Röder, Stock, Neville, Bien \& Rösler, 2002). The researchers varied syntactic processing demands (easy vs. difficult to process word order sequences) and the meaningfulness of sentences (real- vs. pseudo-word sentences). The following material was presented auditorily:

(1) Jetzt wird der Astronaut dem Forscher den Mond beschreiben. (easy redi-word)

(2) Jetzt wird den Mond dem Forscher det Astronaut beschreiben. (alficult red-wowd)

(3) Jetzt witd der Pronasaut dem Schorfer den Rond bereuschen. (easy pseadow word)

(4) Jetzt wird den Rond dem Schorfer der Pronasaut bereuschen. (difficul psendoword)

Playing the sentences of these four conditions backward created the control condition ("backward speech"). For syntax Röder and colleagues reported that syntactic processing in comparison to backward speech resulted in an activation of the left perisylvian region, including the left inferior frontal cortex, and the superior and middle temporal gyri. Semantic in contrast to pseudo-word conditions elicited a stronger activation in both the anterior and the posterior perisylvian cortex. Syntactic difficulty had its strongest effect in the left inferior frontal region. This effect was stronger for real-word than pseudo-word conditions. Thus, semantic and syntactic manipulations interacted within inferior frontal cortex. The authors 
suggested that brain areas show some processing specificity although both language aspects seen to be supported by overlapping brain systems.

Thus, there is no clear anatomic picture about the language system at present. Further research is needed to reveal a complete picture of a neural network for semantic and syntactic integration.

\subsection{Verbal Working memory and pronoun processing}

The use of anaphoric expressions taxes memory resources from a processing point of view because the system has to search for an appropriate antecedent between previously activated memory representations. Proper names are explicit, as they identify the antecedent directly. Thus, a proper name will not trigger any search processes. Pronouns on the other hand are far less explicit because the interpretation depends on e.g. the context, syntactical features, and search processes seem to be obligatory. This idea is substantiated by the fact that more explicit anaphoric expressions are resolved faster and linked with greater likelihood to the appropriate antecedents than less explicit anaphors (Dell, McKoon \& Ratcliff, 1983; Garrod et al. 1994). Studies in which ERPs were recorded during pronoun processing obtained evidence that components of the ERP, particularly the so called left anterior negativity (LAN) and the N400, reflect such processes in working memory load (Kluender \& Kutas, 1993). King and Kutas (1995) compared object and subject relative clauses. They found that object relative clauses, which impose extra load on working memory resources, evoke the LAN. This negativity followed the main verb, i.e. when the system had received all the information to resolve the syntactic structure. Streb and colleagues (1999) compared proper name anaphors and pronouns in parallel and nonparallel structure. A parallel structure means that a subject pronoun refers to a preceding subject noun, as in the sentence "Peter besucht $J$ ulia in der Klinik. Dort hat er dem Arzt eime Frage gestellt." The same holds for an object pronoun which is assigned to an object noun ('Peter besucht Julia in der Klinik. Dort hat die Schwester ihr eine Spritze gegeben.'). Resolving this structure is supposed to be easy because pronoun and noun have the same syntactic function. In contrast, a non-parallel structure, where the pronoun and the preceding antecedent have a different syntactic function, is more difficult to resolve. Therefore, this structure should impose extra load on the system. An example would be: "Peter besucht Julia im Krankenhaus. Dort hat die Schwester ihm das Zimmer gezeigt." or 'Peter besucht Julia im Krankenhaus. Dort hat sie dem Arzt den Besuch vorgestellt.' Streb and colleagues reported two ERP results: (1) Pronoun anaphors evoked a more pronounced 
negativity than proper name anaphors between 270 and $420 \mathrm{~ms}$ latency range over the fromtal cortex electrodes. Another relative negativity occurred between 510 and 600 ms over the parietal sites. (2) Anaphors in nonparallel positions compared to parallel positions were more negative over parietal sites. The authors hypothesized that these two effects are functionally related to discourse resolution including a search in working memory for the suitable antecedent.

Cooke and co-workers (2001) investigated the where of syntactic working memory with fMRI, by presenting sentences differing in grammatical structure (subject- vs. object-relative) and short-term memory demand (short vs. long antecedent gap). Material example would be:

1. [The strange man] in black who [] adored Sue was rather sinister in appearance. (subject-relative, short antecedent gap)

2. [The cowboy] with the bright gold front tooth who [] rescued Julia was adventurous. (subject relative, long antecedent gap)

3. [The flower girl] who Andy punched [] in the arm was five years old. (object-relative, short antecedent gap)

4. [The mess boy] who Janet the very popular hairdresser grabbed [] was extremely hairy. (object-relative, long antecedent gap)

The "[ ]" illustrates the appending noun and the antecedent gap. Subjects were asked to consider whether a male or a female performed the action described in the sentence. There were two main results. First, the right posterior superior temporal cortex showed an activation during processing of long distance antecedent-gap without any grammaticall influence in comparison to a pseudofont baseline. This result was interpreted as showing that this area supports passive short-term memory during sentence comprehension. Second, the left inferior frontal cortex was associated with sentences that featured both an object-relative clause and a long antecedent-gap in comparison to the baseline. This suggests that this region supports the cognitive resources required to maintain long-distance syntactic dependencies during comprehension of grammatically complex sentences. Fiebach, Schlesewsky, and Friederici (2001) found comparable results for German short and long wh-questions differing in their grammatical function (subject vs. object wh-question). 


\subsection{Conclusion}

Psycholinguistic studies (Garrod et al., 1994; Gamham et al., 1995) and ERP-studies showed the involvement of syntactic and semantic information for establishing co-reference between a pronoun and a suitable antecedent. Furthermore, electrophysiological studies showed that the semantic integration starts at about $300 \mathrm{~ms}$ (King \& Kutas, 1997; Schmitt et al., 2002) and syntactic integration at about $400 \mathrm{~ms}$ (Osterhout \& Mobley, 1995; Schmitt et al., 2002). Neuroimaging studies investigated syntactic processing with either detection of grammatical errors in meaningless sentences finding an activated area in the left dorsolateral prefrontal cortex, adjacent to Broca's area (Indefrey et al., 2001), or with varying syntactic processing demands finding an activation of the left perisylvian region, including the left inferior frontal cortex, and the superior and middle temporal gyri (Röder et al., 2002). Semantic processing elicited a stronger activation in both the anterior and the posterior perisylvian cortex.

Working memory demands were measured by varying the difficulty of the sentence structure and comparing full noun anaphors and pronouns (Streb et al., 1999). Parallel structures are supposed to be easier to resolve than nonparallel structures, and therefore demand extra processing. This extra processing (search in working memory) becomes manifested over parietal scalps as a negativity sharing features of an N400 component. Pronouns compared to full name anaphors revealed a more pronounced negativity of left frontal sites, being similar to a LAN and another N400-like effect. Anatomically the right posterior superior temporal cortex is suggested to be involved in processing of long distance compared to short distance antecedent-gap without any grammatical influence (Cooke et al., 2001). Possibly the left inferior frontal cortex supports cognitive resources required to maintain long-distance syntactic dependencies during comprehension of grammatically complex sentences, as has been suggested by Cooke et al. (2001).

\subsection{Research aims}

The present thesis addresses the issue of pronoun processing with a multi-method approach by applying three different methods with on one hand high temporal (i.e. ERP and ERF) and on the other high spatial resolution (i.e. fMRI) enabling us to zoom into two important domains of pronoun processing from a cognitive point of view, viz. the temporal (when) and the spatial (where). Furthermore, comparable materials across experiments were used to 
investigate the issues of syntactic and semantic gender information integration and information integration in combination with verbal working memory processes duning pronoun resolution. This enables us to present a coherent framework between studies for pronoun resolution investigating the when and where of neurocognitive processes in focus.

The research aims in detail:

Chapter 2 scrutinizes the interaction of conceptual/semantic (N400) and syntactic (P600) gender agreement during pronoun processing between an antecedent and it pronoun in sentences about persons or things in German using ERP.

Chapter 3 investigates the localization of neural generators of event-related field (ERF) components for conceptual/semantic and syntactic processes by means of distributed source analyses of MEG data.

Chapter 4 considers the localization of cortical areas and networks of semantic and syntactic processes with MRI, and investigates a neurall network for conceptual/semantic and syntactic integration.

Chapter 5 inspects the interaction of verbal working memory with conceptual/semantic and syntactic gender information processing during pronoun resolution using ERP.

Chapter 6 examines the localization of cortical areas of verbal working memory and possible interactions with conceptual/semantic and syntactic gender information processing during pronoun resolution using fMRI. 


\title{
Pronominal reference in sentences about persons or things:
} An electrophysiological approach

\begin{abstract}
Albstract
Geman pronouns (erMAL/masculine, siemeMaLEmentinine) that refer to a person are detemined by the biological gender: (MALE/FEMALE) and/or syntactic gender (masculine/feminine) of the person. Pronouns (er $\mathrm{r}_{\text {masculatine }}$ sie $_{\text {fentinine, }}$ ) that refer to a thing are determined by the syntactic gender of this thing (Garten (garden) nassume, Tasche (handbag)ieminine. The study aimed to investigate whether senantic integration, syntactic integration, or both are involved in establishing co-reference between pronoun and subject/antecedent in sentences. Here we focused on two event-related potential components: the SPSP 600 , related to syntactic violation and reanalysis, and the N400 component, related to semantic integration problems. In one condition a person was introduced as antecedent and later referved to by pronoun, which either agreed in biologicalsyntactic gender or not (biological/syntactic gender violation). In a second condition, a thing was introduced as santecedent and the corresponding pronoun either agreed in syntactic gender or not (syntactic gender violation). Results at critical pronouns showed a P600 effect for incongruent compated to congrtuent pronouns in both conditions witl a centro-parietal maximum. This affect was latger for the person conpared to the thing condition. We interpreted this finding as reflecting a syntactic integration process that can be influenced by conceptual/semantic and syntactic information of the antecedent type. Furthermore, at the word following the pronoun, we observed an N400 for the thing but not for the person condition. We suggest, supported by the results of a control experiment that this effect reflects contimuous integration processes tor things, whereas for persons the integration seems to be finished at pronoun position.
\end{abstract}

This chapter corresponds to:

Hammer, A., Jansna, B.M. Lamers, M., \& Munte, T.F. (2005). Pronominal reference in sentences about persons or things: An electrophysiological approach, Journal of Cognitive Neuroscience. $17(2), 227-239$. 


\subsection{Introduction}

The phenomenon of establishing co-reference with one item in a sentence, for example a pronoun referring back to an element mentioned earlier in the sentence is a central topic in attempts to understand language comprehension. The question about the underlying cognitive and neural nechanism of the co-reference, as well as the question what kind of information is accessed during comprehension of anaphoric expression have attracted the interest of linguists, psycholinguists and neuroscientists for quite some time and is still a matter of debate (Hankamer \& Sag, 1976; Carreiras, Garnham, Oakhil] \& Cain, 1996; Osterhout et al., 1997; Gordon \& Hendrick, 1998; Schmitt et al., 2002; Swaab, Camblin \& Gordon, 2004). In this study we focus on pronominal reference as exemplified by the sentence "The woman is happy because she is in love. 'or, as in the German sentence 'Die Blume ist schön, weil sie viel Sorne hatte. (The flower is beautful because it ('she') had a lot of sunlight.)' Reading these sentences the comprehender knows that she refers back to the noun. But how does he or she know this? Some psycholinguistic studies have shown the involvement of conceptual/semantic information (Gernsbacher, 1991) to establish the link, others have suggested that it involves syntactic information, i.e. more surface related processing (Gordon \& Hendrick, 1998), or both (Garnham et al., 1995).

Garrod and Sanford (1994) presented a model with two stages for pronoun interpretation (see also Garrod \& Terras, 2000) with (1) a bonding or immediate recovery stage, which is an automatic, more syntax-related process associated with establishing a link between anaphor and a previous expression, and (2) an resolution or immediate integration stage, which is testing and resolving this link. The integration is responsible for evaluating the established link, i.e. by weighting syntactic and semantic discourse information. In case of congruency, the integration proeess finishes with a so-called commitment. In case of incongruency, the integration process fails or is delayed.

We made use of event related potentials (ERPS) to further investigate pronoun processing. ERPS are minute voltage fluctuations of electrical activity produced by newrons in the brain that are recorded from various points on the scalp during the processing of cognitive tasks (Rugg \& Coles, 1995; for language see Kutas, Federmeier, Coulson, King \& Münte, 2000). Their high temporal resolution can be used to zoom into temporal aspects of establishing coreference. Specific ERP components (i.e. the N400 and the P600) can be interpreted as showing semantic and/or syntactic processing. 
Previous ERP studies on pronoun processing reported a variety of findings. For example, Osterhout and Mobley (1995) compared the ERPs to personal pronouns that either matched or mismatched the subject noun in biological gender as in "The aunt heard that she/he had won the lottery.' They found that the mismatching pronouns elicited a P600 for participants judging such sentences as unacceptable. According to the authors, this P600 effect indicated syntactic integration. For participants who found such sentences acceptable, the ERPs to the mismatching trials included a larger negativity compared to matching trials, beginning at approximately $200 \mathrm{~ms}$ after pronoun onset. This negativity was interpreted as a consequence of adding new elements to the existing representation in order to make sense of the mismatching pronoun sentence.

Osterhout and colleagues (1997) investigated reflexive pronouns that referred to (i) definitionally or (ii) stereotypically male or female antecedents. One example would be: (i) The man prepared himself/herself for the interview. (ii) The doctor prepared himself/herself for the operation. Overall, the results showed that ERPs to gender incongruent pronouns were more positive than to congruent pronouns. Moreover, violations of gender definitions were more positive than violations of gender stereotypes. The authors concluded that ERPs are sensitive to violations of gendler based on occupational stereotypes and that the observed ERP response is similar to the $\mathrm{P} 600$ elicited by syntactic anomalies (as shown by Osterhout \& Holcomb, 1992; Hagoort et al., 1993; for a review see Hagoort et al., 1999). The P600 has also been observed in situations requiring the reanalysis of the sentence (Friederici \& Mecklinger, 1996; Münte et al., 1998a).

King and Kutas (1997; see also Kutas et al., 2000) examined the response to pronouns that refer to a stereotypically male or female antecedent noun. The subject noun in the experimental sentence was an occupational title that was more or less likely to be filled by a person of female or male gender, i.e. 'The engineer redesigned the circuit because he/she had detected a flaw.' As one might expect, the paucity of female engineers in reality renders the version of this sentence with 'she' odd at first glance. Readers appear to treat the female pronoun as mismatching. One of the major findings of this study was that mismatching pronouns elicited a larger $\mathrm{N} 400$ component compared to matching pronouns. This $\mathrm{N} 400$ has been shown to be sensitive to semantic integration (Kutas \& Hillyard, 1980, 1983; Kutas \& van Petten, 1994), as well as to discourse integration (St George et al., 1999; van Berkum et al., 1999). The observed effect therefore inclicates an involvement of semantic (world knowledge of stereotypes) and/or discourse processing of words during the integration of the pronoun into the sentence. 
The results by Osterhout and colleagues $(1995$, or 1997) are on a first glance contrary to the results presented by King \& Kutas (1997). However, a direct comparison is difficult due to the difference in design and material. For example, a reflexive pronoun has to be bound in its governing category, whereas a non-reflexive pronoun has to be free. Furthermore, reflexive pronouns, once processed, can only be syntactically correct or incorrect in the given sentence context, whereas personal pronouns offer more degrees of freedom in the interpretation. For example, in case of a mismatch, the parser may leave the sentence level and search within the discourse level for an appropriate antecedent, indexed by the negativity reported by Osterhout \& Mobley (1995). In addition, the observed P600 difference in the Osterhout et al. (1997) study could alternatively be interpreted as an effect of semantic knowledge about gender stereotypes affecting the syntactic process. Then, semantic processing seems to be involved in the resolution process shown by the $\mathrm{P} 600$ variation between stereotype gender violations and clear biological gender violation conditions.

In a recent study Schmitt et al. (2002) examined the separate effect of biological and. syntactic gender violation during pronoun processing. The study was conducted in German because the German language differentiates between biological gender (FEMALE/MALE) and syntactic gender (feminine, masculine, neuter); especially in the cases of diminutives (das Bubchenmaledinewter [the little boy]). Subjects read sentences in which an antecedent

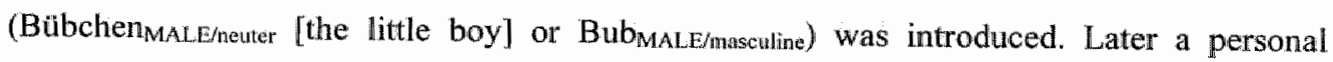
pronoun (er MALE/masculine/ $\mathrm{es}_{\text {neutler }}$ /sie FEMALE/feminine) was presented which either agreed with the antecedent in terms of both gender types, in terms of syntactic gender only, or in terms of biological gender only, or violated both agreements. Overall, the results showed salient P600 effects for pronouns, with the violations being more positive than the non-violations. This indicated that the establishment of reference involves syntactic reanalysis. Furthermore, the authors observed N400 effects in sentences with non-diminutives (violations being more negative than non-violations), but not with diminutives. This showed that conceptual/semantic integration is involved during non-diminutive but not during diminutive pronoun processing; at least it could not be violated.

Taken together, the ERP pronoun studies so far did not reveal a clear pattern with regard to the question what kind of information is used for linking a pronoun to its antecedent. The observation of $\mathrm{N} 400$ or $\mathrm{P} 600$ as indexes for semantic and syntactic processing respectively seems to be dependent on the type of pronoun and the type of antecedent. In addition, the interpretation of the P600 should be broadened. It can be a pure syntactic marker, but it can also be manipulated by semantic information (Münte et al, 1998a). Also, note that all these 
studies focused the ERP analysis on the pronoun position. It is therefore open so far if and how the co-reference process proceeds after pronoun input, a point that will be addressed especially in the second experiment.

\section{Condition Sentence}

\section{P: Person as antecedent}

C. congruent

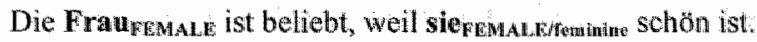

I. incongruent

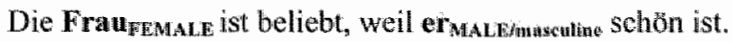

\section{T: Thing as antecedent}

C. congruent

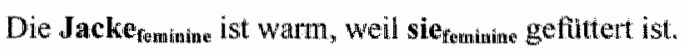

I. incongruent

Die Jacke $\mathbf{f e m i n i n e}_{\text {ist }}$ warm, weil er ${ }_{\text {masculiute }}$ gefüttert ist.

Table 2.1 Example Materials for the experiment. Translation: $P$ : The woman is popular because she/he is beautifil. T: The jacket is warm because she/he is lined. FEMALE = biological gender, masculine $=$ syntactic gender masculine, feminine = syntactic gender feminine.

The aim of the current study is to further investigate the establishment of conceptual/semantic and syntactic gender agreement during pronoun processing. Specifically it was of interest, whether or not these processes differ for persons and things as antecedents. In contrast to English, German nouns referring to things have a syntactic gender, which is independent of any semantic information. Garden, for example, is masculine (der rasculine Garten $_{\text {rnasculine }}$ ), and flower is feminine (die feminine Blume $e_{\text {fenminine }}$ ), while neither of these things are male or female. We assume to tap into unambiguous syntactic processing in this condition, reflected by a $\mathrm{P} 600$. Similar to English, a German noun referring to a person has a gender that is determined by the biological/conceptual gender. A woman, for example, has a female biological gender and a feminine syntactic gender (dieferinine Frauremale [the woman]). Because the two gender types coincide in this case, it is unclear which information is used by the linguistic system to establish co-reference between the pronoun "sie (she)" and the noun "die Frau". It could be semantic information, syntactic information or both. Based on previous ERP findings, we consider three possible types of outcomes. If only biological gender (conceptual/semantic information) plays a role for persons then we would expect an N400 ERP effect in violations of biological gender assignment (according to the results of King and Kutas, 1997). If biological and syntactic gender play a role, we would expect an N400/P600 complex for double violations (as was observed by Schmitt et al., 2002), 
reflecting a more modular and independent semantic and syntactic processing. Alternatively, one could expect a difference between the P600 amplitude in the person compared to the thing pronoun manipulation (as was observed in the stereotype vs. clear biological gender violations by Osterhout et al. (1997) indicating an interaction of semantic and syntactic processing within the $\mathrm{P} 600$ component).

German subjects read sentences in which an antecedent was either a person $(P)$ or a thing $(T)$. This antecedent was introduced in the first phrase. In the second phrase a pronoun was presented which referred back to the antecedent in the first phrase (congruent pronoun $\mathrm{C}$ ) or which was incongruent (I) with the antecedent (examples are given in Table 2.1).
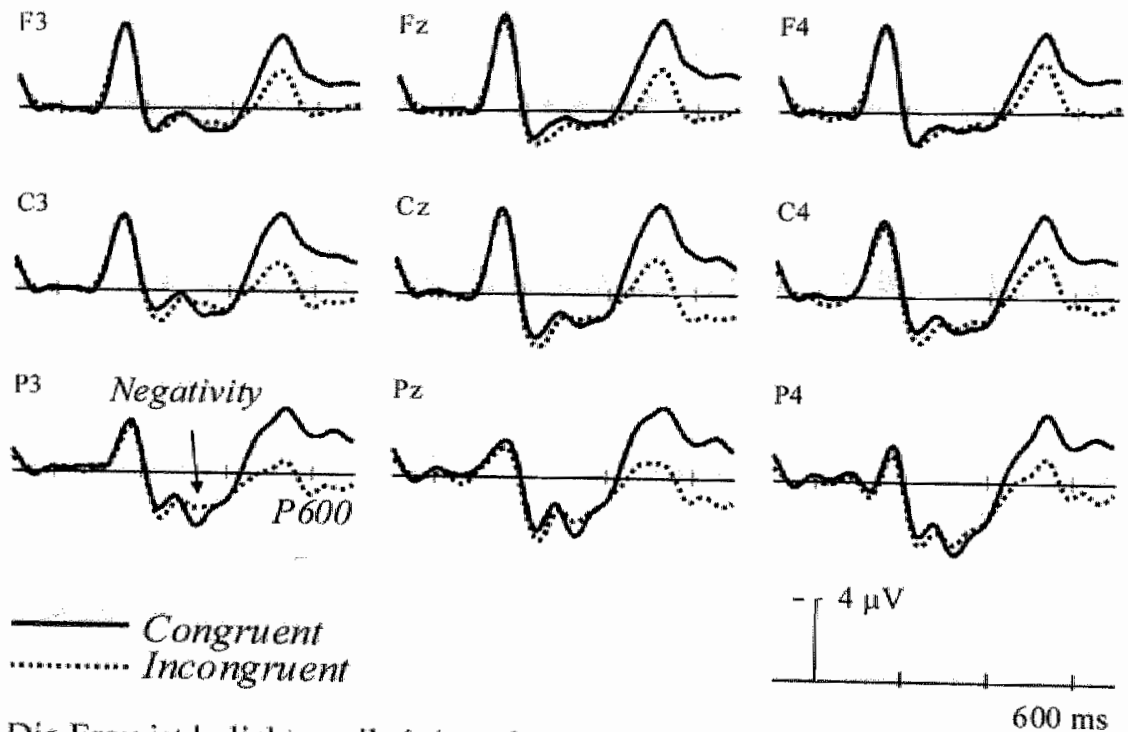

Die Frau ist beliebt, weil sie/er schön ist.

\section{Congruent}

Incongruent

The woman is popular, because she/he is beautiful.

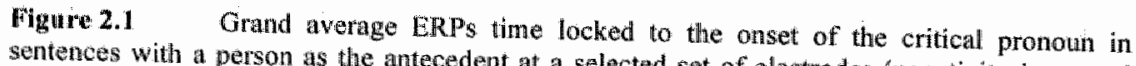
sentences with a person as the antecedent at a selected set of electrodes (negativity is up and each hash mark represents 200 ms of activity in this and in the following figures).

\footnotetext{
In case of modularity this complex has to be identical to the sum of the separate components
(Osterhout \& Nicol, 1999 ). A complex could (Osterhout \& Nicol, 1999). A complex could also show an interaction (in case the sum is not identical
to both component effects in isolation).
} 


\subsection{Results}

\section{Comprehension Task}

The correct performance in the comprehension task was $83 \%$ indicating that the subjects had indeed read and processed the sentences. Mean reaction time was $1800 \mathrm{~ms}$ (SD 350).
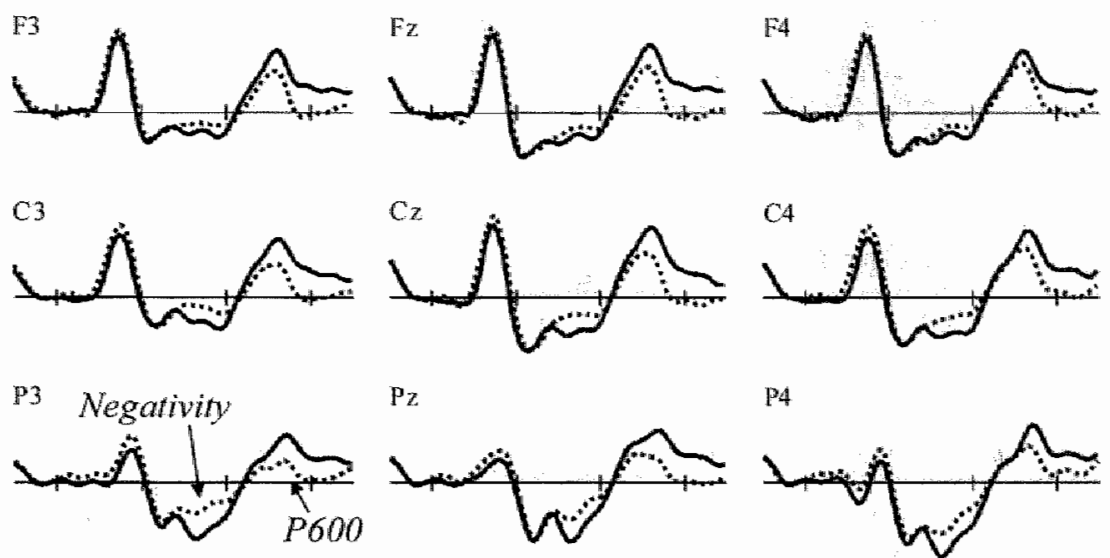

P.

P4
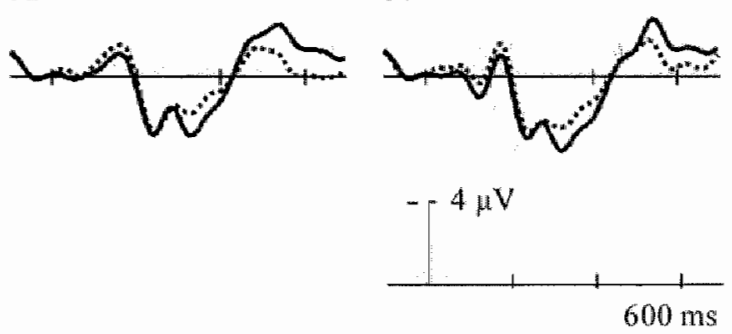

Die Jacke ist warm, weil sie/er gefuittert ist.

The jacket is warm, because it (she/he) is lined.

Figure 2.2 Grand average ERPs time locked to the onset of the critical pronoun in sentences with a thing as the antecedent at a selected set of electrodes.

\section{ERPS}

The grand average ERPs, time-locked to the onset of the critical pronoun, are shown in Figure 2.1 for Person-antecedents, and in Figure 2.2 for Thing-antecedents conditions.

The critical words elicit the N1-P2 complex that is typical for visually presented material, followed by a late negativity. In both antecedent conditions incongruent compared to congruent pronouns are characterized by a more negative waveform in the range of $300-$ $400 \mathrm{~ms}$ after pronoun onset, especially at parietal sites. From $400 \mathrm{~ms}$ after onset and extending well into the ERP to the next word, the ERP to the incongruent stimuli is more positive than that to the congruent pronouns. This P600 effect is larger for sentences with a person compared to a thing as an antecedent. 


\begin{tabular}{|c|c|c|c|c|}
\hline Source & $d r$ & $F$ & $\mathrm{P}(\mathrm{GG})$ & \\
\hline \multicolumn{5}{|l|}{ Omibus ANOVA (29 thectrodes) } \\
\hline Antecedent bype (Person ws. Thing) & 1,15 & 0.08 & .8 & \\
\hline Pronoun type (Congruent ws Incongruent) & 1,15 & 4.59 & $.048^{*}$ & \\
\hline Antecedent type x Pronoun type & $1 ; 15$ & 3.81 & .07 & \\
\hline Antecedent type B Blectrodes & 28,420 & 1.70 & .2 & \\
\hline Pronoun type $x$ Electrodes & 28,420 & 1.92 & 1 & \\
\hline Antecedent lype $\times$ Pronoun type $\times$ Electrodes & 28,420 & 1.02 & 4 & $\therefore$ \\
\hline \multicolumn{5}{|l|}{ Person: Omnibus ANOVA (29 electrodes) } \\
\hline Pronoun type (PC ws. Pl) & 1,15 & 0.92 & .35 & \\
\hline Pronoun type $x$ Electrodes & 28,240 & 2.26 & .08 & \\
\hline \multicolumn{3}{|l|}{ Thing: Omnibus ANOVA (29 ellectrodes) } & & \\
\hline Pronoun type (TC Vs. TO) & 1.15 & 7.92 & $.01^{*}$ & \\
\hline Pronoun type $x$ Electrodes & 28,240 & 1. .08 & .37 & \\
\hline
\end{tabular}

Table 2.2 Gender agreement effects: Mean ERP amplitude ANOVAs in the 300 :- 400 ms Latency Range (Negativity). Note: $\mathrm{PC}=$ congruent Person, $\mathrm{PI}=$ incongruent Person, $\mathrm{TC}=$ congruent Thing, Tl $=$ incongruent Thing; $* \mathrm{p}<0.05 ; * \mathrm{p}<0.01 . \mathrm{GG}=$ Greenhouse Geisser corrected $\mathrm{p}$-value for $\mathrm{F}$ tests with more than one degree of freedom.

\section{Negativity}

Results of the repeated measures ANOVAs on mean amplitudes for the $300-400 \mathrm{~ms}$ timewindow are reported in Table 2.2. The omnibus ANOVA revealed a main effect of Pronoun type with ERPs to incongruent conditions being more negative than congruent conditions. The interaction of Antecedent type with Pronoun type showed a trend. These effects were
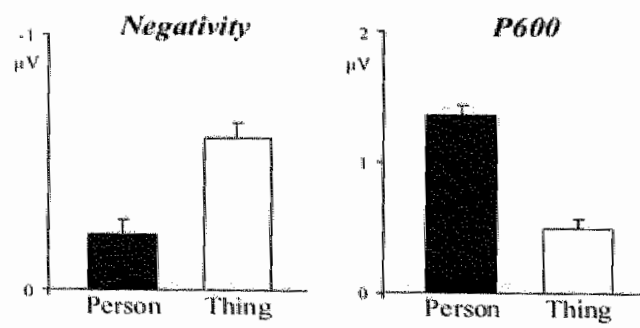

Figure 2.3 Mean amplifudes of difference waves (tincongrwent minus congruent) are demonstrated for the Negativity (time-window $300-400$ ms) at the left and the P600 (time-window $400-700 \mathrm{~ms}$ ) at the right. traced back to the following effects in separate ANOVAs for each Antecedent type. For Thing-antecedents, the ANOVA including 29 electrodes showed a significant main effect of Pronoun type. For Personantecedents, the analysis including 29 electrode sites revealed a trend for Pronoun type $x$ Electrode interaction. The Pronoun type effects (Negativity: incongruent minus congruent) are plotted in Figure 2.3, left panel, separately for the two antecedent types. 


\section{P600}

The omnibus ANOVA on mean amplitudes for the $400-700 \mathrm{~ms}$ time-window revealed no significant main effect for the Antecedent types (see Table 2.3). There was a significant main effect of Pronoun type with ERPs to incongruent pronouns being more positive than those to congruent ones. As visible in the ERP waves, and in Figure 2.3, right panel: the pronoun-type effect (incongruent - congruent) for Person-antecedent was much larger than for Thingantecedents. This difference was statistically reliable, as indicated by the significant interaction of Antecedent type $x$ Pronoun type.

\section{End of sentence effects}

Figure 2.4 shows the grand average ERPs, time-locked to the onset of the critical pronoun up to the end of the sentence for things (left panel) and persons as antecedents (right panel), respectively. In addition to the ERP-pattern described before, Figure 2.4 (left panel) shows a more negative waveform for the word after the pronoun ('gefuittert') in incongruent sentences about things as compared to congruent sentences. This negativity begins at approximately $1000 \mathrm{~ms}$ after the pronoun and lasts to $1300 \mathrm{~ms}$ (corresponding to $400-700 \mathrm{~ms}$ after word onset) with a rightparietal maximum. This pattern is not visible in sentences about persons (see Figure 2.4, right panel). There appears to be a small negativity with a more anterior distribution than the classical N400 (most obvious at electrode F4 and C4).

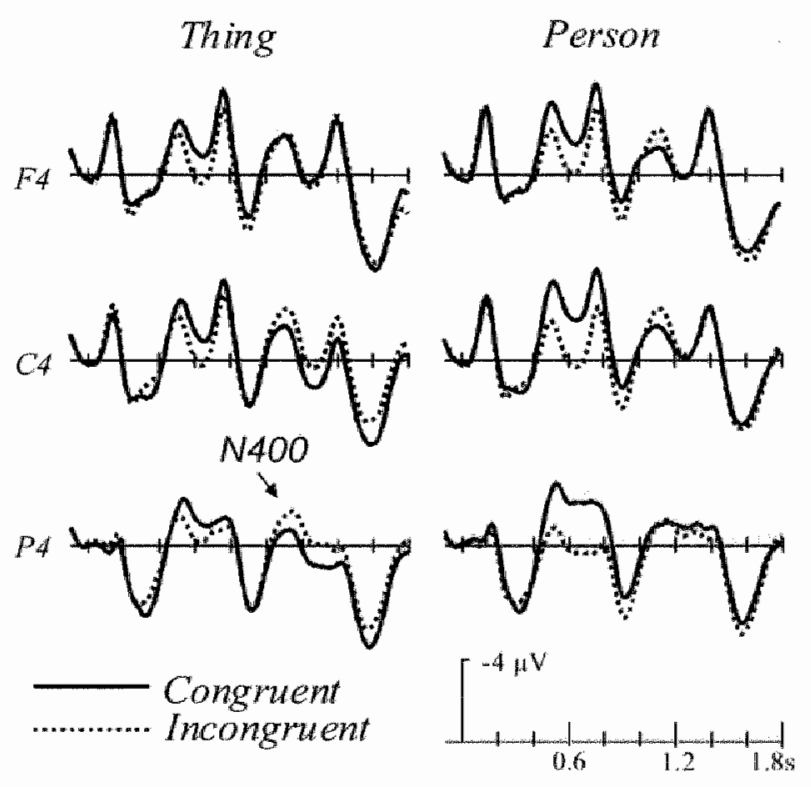

Figure 2.4 Grand average ERPs time locked to the onset of the critical pronoun lasting until the end of the sentence with a thing as the antecedent (left panel) and with a person as antecedent (right panel) at selected set of electrodes. The first word starts at $0 \mathrm{~s}$, the second word at $0.6 \mathrm{~s}$, and the sentence final word at $1.2 \mathrm{~s}$. The same scaling holds for Figure 5 . 


\begin{tabular}{|c|c|c|c|}
\hline Source & dff & $\vec{F}$ & $\mathrm{PrGG}$ \\
\hline \multicolumn{4}{|l|}{ Omnibus AMOVA (29 electruades) } \\
\hline Artecedent ype (Person vs. Thing) & 1,15 & 0.12 & 7 \\
\hline Pronoun type (Congruent vs. Incongruent) & 1,15 & 15.16 & $.001 *$ \\
\hline Antecedent type x Pronoun type & 1,15 & 9.79 & $.007^{*}$ \\
\hline Antecedent type $x$ Electrodes & 28,420 & 1.28 & .3 \\
\hline Pronoun ype x Electrodes & $28 ; 420$ & 7.08 & $.0001 *$ \\
\hline Antecedent type $x$ Pronoun type $x$ Glectrodes & 28,420 & 1.35 & .2 \\
\hline
\end{tabular}

Table 2.3 Gender agrement effects: Mean ERP amplitude ANOVAs in the $400-700 \mathrm{~ms}$ Latency Range (P600). Note: $\mathrm{PC}=$ congruent Person, $\mathrm{Pl}=$ incongruent Person, $\mathrm{TC}=$ congruent Thing, $\mathrm{Tl}=$ incongruent Thing; * $\mathrm{p}<0.05 ; * \mathrm{p}<0.01 . \mathrm{GG}=$ Greenhouse Geisser corrected $\mathrm{p}$-value for $\mathrm{F}$ tests with more than one degree of freedom.

\begin{tabular}{|c|c|c|c|}
\hline Source & df & F & $P(G G)$ \\
\hline \multicolumn{4}{|l|}{ Omnibus ANOVA (29 electrodes) } \\
\hline Antecedent type (Person vs. Thing) & 1,15 & 1.75 & 2 \\
\hline Pronoun type (Congruent vs. Incongruent) & 1,15 & 0.95 & .3 \\
\hline Antecedent type x Pronoun type & 1,15 & 3.58 & .08 \\
\hline Amtecedent type $x$ Electrodes & 28,420 & 2.10 & .08 \\
\hline Pronoun type $x$ Electrodes & 28,420 & 0.97 & .4 \\
\hline Antecedent type $x$ Pronoun type $x$ Electrodes & 28,420 & 1.10 & .4 \\
\hline \multicolumn{4}{|l|}{ Thing: Omnibus ANOVA (29 electrodes) } \\
\hline Pronoun type (TC vs. TI) & 1,15 & 6.36 & $.02 *$ \\
\hline Pronom type $x$ Electrodes & 28,420 & 0.79 & .7 \\
\hline \multicolumn{4}{|l|}{ Thing: Parietal ANOVA (P3, $\left.\mathbf{P}_{2_{4}} \mathbf{P 4}\right)$} \\
\hline Pronoun type (TC vs. Tl) & 1,15 & 12.86 & $.003^{* * *}$ \\
\hline Pronoun type $x$ Electrodes & 2,30 & 0.09 & .9 \\
\hline \multicolumn{4}{|l|}{ Person: Omuibus ANOVA (29 electrodes) } \\
\hline Pronoun type (PC vs. PI) & 1,15 & 1.03 & .3 \\
\hline Pronoun type x Electrodes & 28,420 & 1.26 & 3 \\
\hline \multicolumn{4}{|l|}{ Person: Parietal ANOVA (P3, Pa, P4) } \\
\hline Pronotin type (PC vs. PI) & 1,15 & 0.31 & .6 \\
\hline Pronoun type $\mathrm{x}$ Electrodes & 2,30 & 1.03 & .4 \\
\hline Pronoun type x Electrodes & 2,30 & & \\
\hline
\end{tabular}

Tabile 2.4 Gender agreement effects: Mean ERP amplitude ANOVAs in the $1000-1300 \mathrm{~ms}$ Latency Range after onset of the critical pronoun $(\mathrm{N} 400)$. Note: $\mathrm{PC}=$ congruent Person. $\mathrm{PI}=$ incongruent $\mathrm{Person}, \mathrm{TC}=$ congtuent Thing. $T=$ incongruent Thing; $p<0.05 ; * p<0.01, G G=$ Greenhouse Geisser corrected $p$-value
for $F$ tests with more than one degree of freedom. 
Table 2.4 revealed that our pronoun manipulation in an omnibus ANOVA crossing factors Antecedent-type, Pronoun-type, and Electrodes did not show significant effects in the specified time-window, but a trend. Separate analysis for Thing-sentences showed a significant main effect for Pronoun-type in an Omnibus ANOVA and in a Parietal ANOVA, indicating an N400 (400-700 ms) effect. Analysis for Person-sentences revealed no significant effects in this time-window, indicating that the violation did not affect the processing of the word after the pronoun in this condition. An additional ANOVA on right fronto-central sites (C4, F4) did not reveal a significant main effect for Congruency (Pc vs. Pi) for Person sentences $\mathbb{F}(1,15)=1.17, \mathrm{p}<.3$.

\section{Discussion I}

The aim of this experiment was to investigate the role of conceptual/semantic and syntactic information in the process of establishing co-reference between a pronoun and its antecedent. In German things have a clear syntactic gender. We therefore expected to tap into pure syntactic processing during the violation of the pronoun gender, which should be reflected by a P600. In contrast, the person antecedents within this experiment have an unambiguous biological gender, which coincides with syntactic gender. Thus, in the case of a person antecedent conceptual/semantic information (viz. the biological gender) and syntactic information might be used to establish co-reference. We found a more positive ERP for incongruent compared to congruent pronoun types. This P600 effect was larger for person than for thing-antecedent type sentences. If we take only the owerall $\mathrm{P} 600$ pattern into account, these findings suggest that establishing co-reference might be a syntactic process in general. Moreover, the fact that the P600 was larger for persons compared to thingantecedents shows that the syntactic process, in this case the reanalysis of the sentence, might interact with semantics if semantic knowledge is available. Besides, we found a small negativity at left parietal sites in the time window between 300 and $400 \mathrm{~ms}$ alter pronoun onset. This observed negativity, however, did not resemble a standard N400 effect, which usually is more prominent over the right hemisphere and lasts longer (Kutas \& Hillyard, 1983, Münte et al., 2001; for a review see Kutas \& van Petten, 1994). Therefore, we can only speculate here about a possible explanation. Since we found this effect within the thingcondition - where we expected pure syntactic gender information processing - this negativity could be due to a search for an actor outside the sentence in case of an incongruent pronoun, i.e. leaving sentence level and search within the discourse model (from a cognitive point of view comparable to the anterior negative effect found by Van Berkum, Brown, Hagoort \& 
Zwitserlood (2003) for discourse-referential ambiguity). Thus, it has to be considered that the pre-P600 negativity results could be interacting with the following P600. In other words, the P600 to Thing-pronouns might not really be smaller than that to Person-pronouns, rather it might be the larger negativity to Thing-pronouns that "pulls down" the subsequent P600 (i.e., this might be a component overlap issue rather than a pure $\mathrm{P} 600$ issue).

We also observed an unexpected $\mathrm{N} 400$ at the word following the pronoun. This N400 was significant for thing-antecedent types, and was absent for person-antecedent types. As a possible interpretation we suggest that this pattern reflects two types of processing: In the person case, the gender violation at pronoun position is so severe (syntactically and biologically bonding and resolution is signaling violation) that the reader/parser stops further attempts to integrate this mismatching pronoun immediately. Thus, no ellectrophysiological signs of integration attempts were found for the following word. In the thing case, the gender violation is purely syntactical and therefore the bonding process signals "violation" "but due to the lack of any semantic information, a resolution does not take place at pronoun position. The reader/parser is waiting for new incoming information to solve this incongruency. For example, the parser waits for (anticipates) a word that is related to any alternative antecedent in the discourse. But when the word matches the thing-antecedent in meaning (in our example: 'gefüttert'), the comprehension system is unable to link the pronoun to any alternative antecedent. Therefore the integration process of the incongruent pronoun finally signals violation at word position after the pronoun indexed by the N400. To test this assumption, we conducted a second experiment that manipulated the word following the pronoun and added discourse information.

\section{Experiment 2: Pronoun integration depending on context information}

We performed this follow-up experiment for two reasons. First, we wanted to replicate the observed N400 pattern at the word following the pronoun for the thing-antecedent sentence types. In addition, we wanted to test our assumption that in case of pure syntactic gender violation as in our thing sentences, the system keeps on looking for a meaning in the sentence or alternatively within the discourse. We used thing-antecedents and corresponding pronoun violations that were identical to those used in the first experiment. A context was created by adding a sentence preceding the target sentence. This sentence always introduced a person as an additional antecedent, i.e. a potential alternative to the thing-antecedent. This person could either have the same syntactic gender as the thing in the second sentence (ambiguous; A) or 
not. Therefore the person was either congruent $(\mathrm{C})$ or incongruent (I) to the pronoun $\mathrm{In}$ addition, the word following the pronoun could either refer in meaning to the thing or to the alternative discourse person, leading to 6 experimental conditions in total (Table 2.5).

\begin{tabular}{|c|c|c|}
\hline Condition & Sentence & $\therefore 9$ \\
\hline \multicolumn{3}{|c|}{ CT: Content Thing } \\
\hline A ambiguous & 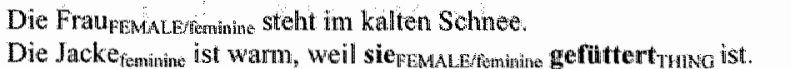 & \\
\hline$C$. congruent & 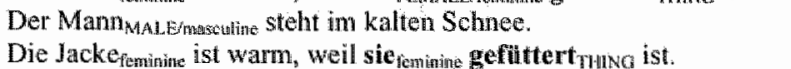 & \\
\hline 1. incongruent & 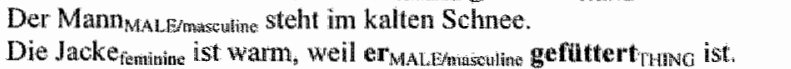 & \\
\hline CP: Content P & & \\
\hline A ambignous & 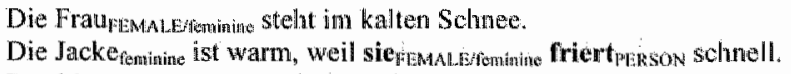 & \\
\hline C. congruent & 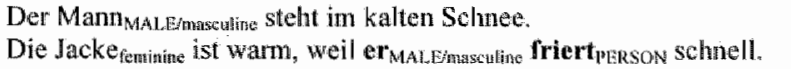 & \\
\hline 1. incongruent & 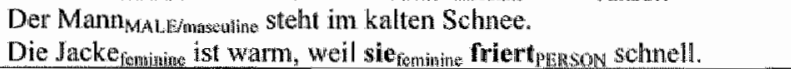 & \\
\hline
\end{tabular}

Table 2.5 Example Materials for the follow-up experiment. Translation: The man/woman stands in the cold snow. The jacket is warm because she/he is lined/freezes fast. FEMALE = biological gender fenale, MALE = biological gender male; masculine = syntactic gender masculine, feminine = syntactic gender feminine, THINGi $=$ word refers to the thing in meaning; PERSON $=$ word refers to the person in meaning.

In our example the word 'gefüttert' [lined] can only refer to the thing-antecedent 'Jacke" (context: thing; CT) within the same sentence, whereas the word 'friert" [freezes] can only refer to the person (context: person; CP) - to the alternative antecedent outside the sentence. The underlying rationale is the following. If the pronoun matches either the within- or outside-sentence antecedent (CTA and CPA), there is no violation between the word following the pronoun and antecedent, and the process of co-reference is finished at this word position (ERP baseline condition). In the case of a pronoun-thing antecedent violation (CTI), (i) the parser waits for new input related to either the within- or a controlled outside-sentence antecedent. (ii) The parser checks the match between the new word and pronoun, and (iii) between new word and within-sentence antecedent. (iv) In case of no-match, the parser looks for alternative antecedents - i.e. somewhere outside the sentence (experiment 1), or in the preceding sentence (experiment 2). Thus, the baseline conditions are those cases where the pronoun can refer back either to the thing-antecedent or the additional person-antecedent. Also, when the word following the pronoun refers back to the discourse antecedent, all words can be processed on-line without any problems. In contrast, an N400 is expected in two cases: a) if the pronoun does not match the thing-antecedent, AND the following word does not 
match the discourse-antecedent (CTI) or b) if the following word does match the thingantecedent and the protioun, but does not match the discourse-antecedent in meaning (CTC).

\section{Results Experiment 2}

The grand averages ERPs, time-locked to the pronoun up to the end of the sentence for the thing-context (left panel, $\mathrm{CT}$ ) and for the person-context (right panel, CP) are shown in Figure 2.5. Around $1000 \mathrm{~ms}$ after the pronoun (equivalent to $400 \mathrm{~ms}$ after word following the pronoun) all conditions revealed a deflection towards negativity lasting until $1300 \mathrm{~ms}$ with a right-parietal maximum. In this $1000-1300 \mathrm{~ms}$ time window this deflection was different within the thing context for pronoun types with CTI being more negative than CTC than CTA. This suggested a modulation of the N400 component. This N400 pattern is not visible when the meaning of the word following the pronoun refers to the person. However, there seems to be a trend towards a negativity with more anterior distribution for the person context. The statistical analysis for the N400 time window $(1000-1300 \mathrm{~ms})$ for the word following the pronoun is reported in Table 2.6. The ommibus repeated measures ANOVA revealed no significant main effects. A more specific parietal analysis resulted in a significant main effect for Pronoun-types indicating indeed a manipulation of the N400. Separate

$C T$
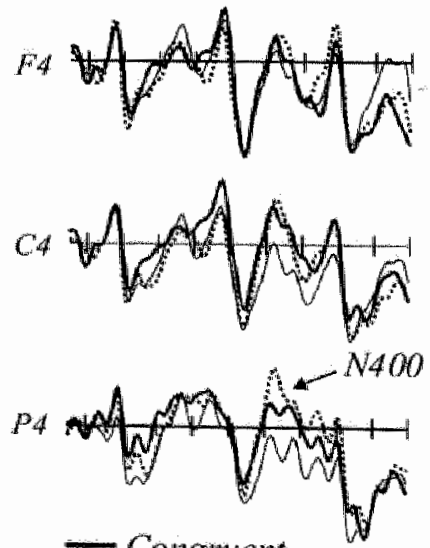

- Congwent

Ambigwous

........ Incongrwent
$C P$
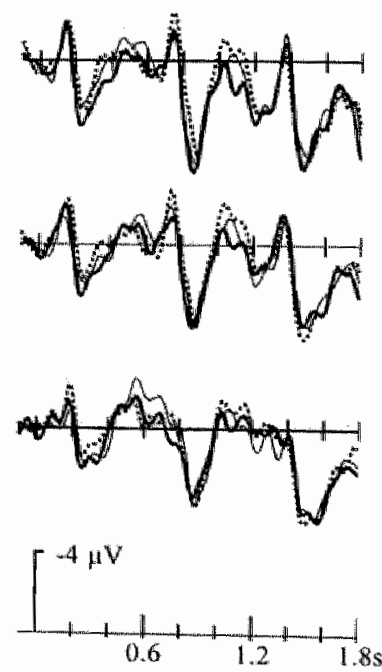

Figure 2.5 Grand average ERPs time locked to the onset of the pronoun lasting until the end of the thing-context (CT) sentence (left panel) and the person-context (CP) sentence (right panel) at a selected set of electrodes.

planned comparison of Pronoun types for the Thing-Context within the specified time-window for electrode site P4 (the site that showed most clearly the N400 in experiment 1) revealed a significant difference between Pronoun-types. Additional planned pair-wise comparisons revealed significant differences between CTA and CTI ( $t_{15}$; one-tailed $=53.51, p<.003$ ) and between CTA and CTC ( $t_{15}$; one-tailed $=$ $22.04, \mathrm{p}<.04)$. In contrast, there was no difference between Pronoun-types for the Person- 
context conditions. These results indicate that there was an N400 effect for pronoun-types within the thing-context (see Figure 2.5, left panel), whereas this manipulation did not affect the word after the pronoun within the person-context (Figure 2.5, right panel). Because of a possible anterior negativity for the Person-context we performed an additional separate frontal ANOVA the Person-context which crossed the factors Pronoun type 3 ; ambiguous vs. congruent vs. incongruent) and 'Electrode sites' ( 2 levels, C4, F4). This analysis resulted in no main effect for the Person-Context; $F(2,30)=0.55 ; \mathrm{p}<.6$.

\begin{tabular}{|c|c|c|c|}
\hline Source & df & F & $\mathrm{P}(\mathrm{GG})$ \\
\hline \multicolumn{4}{|l|}{ Omnibus ANOVA (30 electrodes) } \\
\hline Context (Person- vs. Thing-antecedent) & 1,15 & 0.05 & .8 \\
\hline Pronoun (A vs. C vs. I) & 2,30 & 2.25 & .1 \\
\hline Context $x$ Pronoun & 2,30 & 0.56 & .6 \\
\hline Context $x$ Electrodes & 29,435 & 1.00 & .4 \\
\hline Pronoun $x$ Electrodes & 58,870 & 1.51 & 2 \\
\hline Context $x$ Pronoun $x$ Electrodes & 58,870 & 0.94 & .5 \\
\hline \multicolumn{4}{|l|}{ Parietall ANOVA $\left(\mathbb{P 3}, \mathbf{P}_{Z}, \mathbb{P}_{4}\right)$} \\
\hline Context (Person- vs. Thing-antecedent) & 1,15 & 0.10 & .8 \\
\hline Pronoun (A vs. C vs. I) & 2,30 & 5.10 & $.01^{*}$ \\
\hline Context x Pronoun & 2,30 & 0.20 & .8 \\
\hline Context $\times$ Electrodes & 2,30 & 0.01 & .9 \\
\hline Pronoun $x$ Electrodes & 4,60 & 1.40 & 3 \\
\hline Context $x$ Pronoun $x$ Electrodes & 4,60 & 2.23 & .09 \\
\hline \multicolumn{4}{|l|}{ Single electrode $(\mathbb{P} 4)$} \\
\hline Context (Person- vs. Thing-antecedent) & $1, \mathbb{1} 5$ & 0.17 & .7 \\
\hline Pronoun (A vs. C ws. I) & 2,30 & 6.48 & $.006^{* * *}$ \\
\hline Context $x$ Pronoun & 2,30 & 0,93 & 4 \\
\hline \multicolumn{4}{|l|}{ Planned Comparison P4" Thing-Context } \\
\hline Pronoun (A vs. C vs. V) & 2,30 & 4. 13 & $.02^{\circ}$ \\
\hline \multicolumn{4}{|l|}{ Planned Comparison P4: Person-Context } \\
\hline Pronoun (A vs. C vs. D) & 2,30 & 0.76 & .8 \\
\hline
\end{tabular}

Table 2.6 Mean ERP amplitude ANOVAs in the 1000 - 1300 ms Latency Range after onset of the pronoun (N400 at following word) for the second experiment. Note: $A=$ ambiguous; $C$ congruent, I incongruent; $* \mathrm{p}<0.05 ; * \mathrm{p}<0.01 . \mathrm{GO}=$ Greenhouse Geisser contected pwalue for $\mathrm{F}$ tests with more than one degree of freedom.

\section{Discussion 2}

The aim of the second experiment was to replicate the observed N400 at the word following the pronoun that has a syntactic gender violation with the within sentence thing-antecedent. In addition, to test our post hoc explanation for the effect, we systematically added out-of sentence context and manipulated the word following the pronoun. As a replication of experiment 1 , we observed a more negative effect on the word following the pronoun within 
an incongrutent sentence about things, but no effects for pronouns referring to a person out-of the sentence. Jror the thing-context, the incongrient condition was more negative than the congruent condition (see Figure 2.5). This effect could be interpreted as an ongoing integration process in order to get the meaning of the pronoun correct. This revision might be characterized by a searching process for an alternative antecedent in the discourse. Therefore, the parser waits for new information, in order to solve this incongruity, i.e. expecting a relation to an alternative antecedent in the discourse. The $\mathrm{N} 400$ occurs if this process fails. It fails a) if the pronoun does not match the thing-antecedent, AND the following word does not match the discourse-antecedent in meaning (CTI), or b) if the following word does match in meaning with the thing-antecedert and the pronoun, but does not match with the discourse-antecedent in meaning (CTC). In contrast, in the person-case a revision does not take place at the word following the pronoun, indicating that the integration process for these pronouns seems to be finished right after pronoun processing due to a reliable declining of bonding/resolution of pronoun and person-antecedent.

\subsection{General Discussion}

We conducted two experiments to investigate the role of syntactic and semantic information in the establishment of pronoun resolution in sentence reading. In experiment 1, congruency was manipulated in sentences with either Persons or Things as antecedents. For incongruent sentences with person as well as thing-antecedents we found a clear P600 effect at pronoun position indicating an involvement of syntactic information and/or sentence reanalysis during pronoun processing. This P600 finding is in line with other ERP findings (Osterhout \& Mobley, 1995; Osterhout et al., 1997, Schmitt et al., 2002).

The P600 was significantly stronger in sentences with a person than a thing as an antecedent. Granted the assumption that the size of the P600 effect reflects the effort of reanalyzing and/or syntactically repairing the sentence at pronoun position, this result indicates a larger effort to reanalyze and/or repair in sentences with persons (in which both types of gender are violated) compared to things (violation of syntactic information only). The observed difference in P600 effects might not just be an indicator for syntactic violations. Rather, it might be a response to reanalysis of the sentence as well, including both syntactic and semantic aspects (for a discussion see Münte et al., 1998a). Granted the assumption that the difference in size is related to a component-overlap of the pre-P600 negativity (i.e. Which 
"pulls down" the P600 to Thing-pronoms) as indicated in Discussion 1, this result could indicate that already at the pronoun position the comprehension system is prepared to search for a possible animate discourse antecedent, i.e. another person. This possibility is confirmed by the unexpected finding in experiment 1 . We found an $\mathrm{N} 400$ effect in incongruent sentences about things at the word following the pronoun, indicating ongoing semantic integration/reanalysis. This $\mathrm{N} 400$, however, was only observed for sentences with a thing as within sentence antecedent, and not when this was a person.

In experiment 2 we replicated and investigated this finding further. We provided an additional discourse sentence with either a female or a male person as subject as an alternative antecedent preceding the thing-sentences of the first experiment. Furthermore we manipulated the word after the pronoun in such a way that it either belonged to the thingantecedent (Thing-Context) or to the new person-antecedent (Person-Context) in the preceding discourse sentence. Thus, the pronoun in the Thing-sentence could either refer back ambiguously to the thing-antecedent or to the person-antecedent or congruently/incongruently to the thing-antecedent or finally congruently/incongruently to the person-antecedent. Our ERP data revealed a clear N400 effect on the word following the pronoun for the thing-context whenever there was a violation, but not in the person-context. The N400 has been shown to be sensitive to semantic integration (Kutas \& van Petten, 1994). Thus, our N400 effect indicates that there was still an ongoing integration process on the word following the pronoun if it referred to the thing in meaning, but not if it referred to the person. Furthermore, based on the observed N400 effect size this integration can be differentiated depending on the given context. For example, the N400 was of medium size when the pronoun and the following word agreed with the within sentence antecedent, but when the following word did not fit in meaning to the discourse antecedent. Based on the type of violation in this condition, we suggest that this medium sized N400 does not reflect an integration problem within the sentence, but rather a violation of discourse processing. The N400 was large when the pronoun did not agree in gender with the within sentence antecedent and the following word did not agree in meaning with the discourse antecedent. The larger N400 indicates a sort of double violation, i.e. a failure of agreement within the sentence, and within the discourse. So far, we cannot say which of the agreement processes comes first, or has priority. It seems as if both - the within and between sentence integrations - take place as default. So, in case of no agreement the parser anticipates for a disjoint coreferential relation preferably to a person-antecedent because of the preference for pronouns to refer to persons. In order to anticipate incongruent thing-sentences the language system 
expects a word with such a semantic content that the pronoun fits referring to the discourseperson. "Gefuttert" is not such a word and therefore there is a lexical integration problem of this word. For the person context there is no N400 for the word following the pronoun. In this case the comprehersion system is not hampered in searching for an alternative antecedent after the pronoun because this word refers in meaning to the person-antecedent and a disjoint co-referential relation is established.

Taken together, the data of the two experiments show that at pronoun position violations in syntactic and in a combination of syntactic and biological gender violation are reflected in the P600. The larger P600 for person compared to thing antecedent types shows that the P600 is sensitive to the underlying semantic information of the antecedent (here, whether or not it has biological gender). The observed $\mathrm{N} 400$ on the word following the pronoun in case of thing but not person antecedent types within the same sentence indicates, for the first time that after violations of the pronoun the parser tries to integrate at later sentence positions, and it does so differently for each type of antecedent.

\subsection{Methods}

\section{Experiment I}

\section{Subjects}

Twenty-one subjects volunteered for paid participation in the experiment. Five datasets could not be used due to too many artifacts or technical problems. The final population of subjects comprised sixteen native speakers of German, age range 20 to 30 , mean age 24.6 years, 8 wonen. All subjects had normal or corrected-to-normal vision, were right-handed according to self-report and neurologically healthy.

\section{Material}

Two-hundred-forty sentences, 120 about persons and 120 about things were constructed. Each sentence had two clauses. The first clause in each sentence was the main clause, which described a state of a person or a thing. The person or the thing was the subject of the main clause, and formed the antecedent of the pronoun following later. Care was taken to guarantee equal word frequencies for persons and things using the CELEX-database (Baayen, Piepenbrock, \& Gulikers, 1995). The second clause was a subordinate clause introduced by the conjunction weil (because). This conjunction was followed by the critical 
word, a pronoun referring to the person or the thing. All sentences were then copied and the congruent pronoun was replaced by an incongruent pronoun (see Table 2.1 for examples). This resulted in a total of 480 sentences and in four conditions with 120 sentences per condition: (PC) A person as an antecedent with a congruent pronoun, (PI) a person with an incongruent pronoun and (TC) a thing as an antecedent with a congruent pronoun. (T1) a thing with an incongruent pronoun. The sentences were pseudo-randomized over four blocks, such that each condition appeared thirty times in each block, and that the sentences with the same subjects in the main clause were not in the same block.

In order to keep participants attentive, and to guarantee that they read the sentences, every 10 \pm 5 sentences one of 48 comprehension questions was presented. These questions pertained to the immediately preceding set of $10 \pm 5$ sentences and required a yes/no response. Subjects had to press one out of two buttons for "yes" or "no". This task was independent of the pronoun manipulation.

The words were presented visually on a back projection screen at a distance of $100 \mathrm{~cm}$. Critical words subtended 0.5 degrees of visual angle in height and 1.2 degrees in width.

\section{Procedure}

Participants were tested in a dimly lit sound-attenuating room. They were seated in a comfortable chair. Their instructions were to read the sentences carefully, and to answer the questions related to the sentences from time to time. They were free to blink between the sentences and while answering the questions, but were instructed to fixate on the screen, to be as relaxed as possible and to avoid all movement while reading the sentences.

A trial started with a fixation cross in the middle of the screen, presented for $2050 \mathrm{~ms}$ with a $300 \mathrm{~ms}$ inter-stimulus interval, and was followed by the word-by-word presentation of the sentence. Each word was presented for $350 \mathrm{~ms}$ with a $250 \mathrm{~ms}$ inter-stimulus interval. Sentence ending words were presented with a dot indicating the end of a sentence, followed by a blank screen for $850 \mathrm{~ms}$.

Questions were presented on the screen for $4 \mathrm{~s}$. One block lasted approximately 15 minutes. The entire experiment, including instructions, electrode application and removal took about 2.5 hours.

\section{EEG recording and analysis}

Electroencephalography (EEG) signals were registered with a digitization rate of $254 \mathrm{~Hz}$ and filtered with a band pass of DC to $50 \mathrm{~Hz}$. Twenty-nine tin electrodes were applied according 
to the $10 / 20$ system (positions: $\mathrm{Fz}, \mathrm{Cz}, \mathrm{PZ}, \mathrm{Oz}, \mathrm{Iz}, \mathrm{Fpl} / 2, \mathrm{~F} 3 / 4, \mathrm{~F} 7 / 8, \mathrm{~T} 7 / 8, \mathrm{C} 3 / 4, \mathrm{P} 3 / 4$, $09 / 10, \mathrm{P} 7 / \mathrm{P} 8, \mathrm{Fcl} / 2, \mathrm{Cp} 1 / 2, \mathrm{Po3} / 4, \mathrm{Po} 7 / 8$ ). Bio-signals were recorded with a left mastoid reference, and were offline re-referenced to the mean of the activity at the two mastoids. Horizontal eye movements were recorded from an electrode behind the right lateral orbital ridge and referenced to an electrode at the left orbital ridge. Eye blinks and vertical movements were recorded from an electrode below the right orbital limb and referenced to the left mastoid. EEG and EOG signals were amplified using a 32-channel Synamps amplifier. All electrode impedances (EEG and EOG) were kept below $5 \mathrm{kOhm}$.

From the continuous signal epochs were created of $1006 \mathrm{~ms}$ starting $100 \mathrm{~ms}$ prior to pronoun onset. These epochs were monitored for artifacts, such as eye-movements, by an automated procedure. Trials including artifacts were rejected from further analysis. By averaging the remaining artifact-free epochs per condition the Event-related potentials (ERPs) were derived. The mean number of trials contributing to the average was 107 (PC and TC conditions) and 108 (PI and TI conditions). Waveforms were quantified by mean amplitude measures to assess $\mathrm{N} 400$ (time window 300 to $400 \mathrm{~ms}$ ) and P600 (400 to $700 \mathrm{~ms}$ ) components. Note that the N400 time window diverges from the standard N400 time window (usually between 300 and $500 \mathrm{~ms}$ ) in order to avoid overlap with the P600 time window. These windows were derived from previous pronoun experiments of our group (Schmitt et al., 2002) and corroborated by visual inspection of the grand average ERPS. These measures were subjected to repeated measures analysis of variance that crossed the factors Antecedent type (2; person vs. thing), Pronoun type ( 2 ; congruent vs. incongruent) and 'Electrode sites' (29 levels). The Greenhouse-Geisser correction for inhomogeneity of covariance was applied whenever an evaluated effect had more than one degree of freedom in the numerator. Reported p-values are corrected. Additionally, we compared the mean amplitudes of conditions in the N400 time window by separate analyses for Pronoun type (PC vs. PI, and TC ws. TH). Finally ERPS were averaged for $2012 \mathrm{~ms}$ epochs with a $100-\mathrm{ms}$ pre-stimulus interval in order to analyze the processing of the end of the sentence. These measures were subjected to repeated analysis of variance following the same logic as above and an additional analysis for the parietal line (P3, Pz, P4) in a time window from 1000 to $1300 \mathrm{~ms}$ (corresponding to a $400-700 \mathrm{~ms}$ time-window for the word after the pronoun).

\section{Experiment 2}

The second experiment followed the same procedure as the former experiment with regard to the timing of stimulus display, and EEG recording (despite one additional electrode $-\mathrm{F} p \mathrm{pz}$ ). 


\section{Subjects}

Twenty subjects volunteered for paid participation in the second experiment. Four datasets were rejected due to too many artifacts. The final population of subjects consisted of sixteen. native German speaker, age range 20 to 27 , mean age 22.0 years, 11 women. All subjects had normal or corrected-to-normal vision, were right-handed according to self-report and neurologically healthy.

\section{Material}

Fifty congruent thing-sentences of the former experiment were selected. Additionally we created two types of discourse sentences with a person as a subject who had either the same gender as the thing (ambiguous pronoun resolution, CTA and CPA) or not (see Table 2.5 for example material). If context person and thing-antecedent had different gender, the pronoun 'he' could refer back to the man and the pronoun "she' to the jacket in our example. Furthermore we replaced the word following the pronoun ('gefüttert' lined) with a word ("friert' freezes) that resolves the sentence in such a way that the second clause belongs to the person in the added context-sentence. At that point of sentence processing the re-reference of the pronoun is clear: either being congruent (CTC) or incongruent (CTI) to the thing ( Jacke and gefüttert' jacket and lined) or either congruent (CPC) or incongruent $\mathrm{CPI}$ ) to the person ("Mann/Frau and friert" man/woman and freezes). Thus, the material resulted finally in six different conditions. The material was distributed across two lists remaining 25 sentences per condition in one list, counterbalancing the thing-antecedent and the word following the pronoun. On a given list each thing-antecedent appeared three times with one person-context and two thing-context words following the pronoun or vice versa. Sentences on a list were pseudo-randomized over two blocks in such a way that the same thing-antecedents were kept apart as far as possible. We measured eight subjects per list. They were pooled again for later analyses.

\section{Analyses}

The time window for the mean amplinde analyses of the N400 was 1000-1300 ms after pronoun onset, as in experiment 1 . The mean amplitude values were subjected to repeated measures analysis of variance that crossed the factors Context (2; Person- vs. ThingReference), Pronoun type (3; ambiguous vs. congruent vs. incongruent) and 'Electrode sites' (30 levels). Additionally, we compared the mean amplitudes of conditions in the N400 time 
window by separate analysis for the parietal line (P3, PZ, P4), and by means of planned comparisons between conditions for the single electrode P4. P4 was singled out because of the observed maximum of the effect in experiment 1 , and because of the expectation of a right centro-parietal hemispheric maximum for the $N 400$ amplitude. Reported p-values are corrected via Greenhouse-Geisser correction for inhomogeneity of covariance. 


\title{
Pronoun resolution in sentences about persons or things investigated by Magnetoencephalography
}

\begin{abstract}
The previous event-related potential (ERP) study revealed a clear pattern of pronoun resolution for person and thing antecedents in German. For incongruent sentences with person as well as thing-antecedents, we found a P600 effect at pronoun position indicaling an involvement of syntactic information and/or sentence reanalysis during pronoun processing. The P600 was significantlly larger in sentences with a person than a thing as antecedent. This observed difference in $\mathrm{P} 600$ effects was interpreted as a possible response to reanalysis of the sentence, including both syntactic and semantic aspects. We used magnetoencephalographic recordings to gain deeper insights into the spatial domain of this cognitive process. Statistical analysis did not reveal reliable differences for the event-related fields (ERF) between incongruent and congruent pronouns for both antecedent types. This result was discussed as demonstrating different outcomes for ERP and ERF data due to different characteristics of the two methods, i.e. measuring tangential vs. radial sources or widely distributed sources for higher cognitive processes.
\end{abstract}




\subsection{Introduction}

In order to gain further insights into the temporal and spatial domain of pronoun processing, magnetoencephalography (MEG) signals were acquired simultaneously with the EEG signals described in the first experiment. As mentioned in more detail before, in German a Person carries two types of gender information; namely biological/conceptual and corresponding syntactic gender information. In contrast, things are only characterized by (arbitrary) syntactic gender information. This characteristic of the German language enables the experimenter to systematically manipulate conceptual/biological and syntactic gender information during the process of pronoun processing. A pronoun, which refers back to a person may be rereferenced by conceptual and/or syntactic information, i.e. as exemplified by the sentence

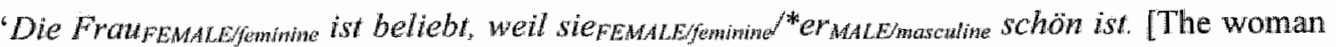
is popular because she/*he is beautiful.]". The "*" marks the incongruent pronoun. On the other thand, a pronoun, which refers back to a thing can solely be re-referenced by syntactic

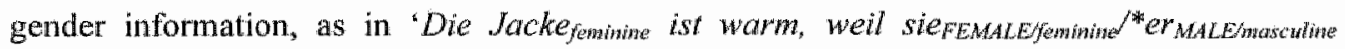
gefuittert ist. [The jacket is warm because (it) she/*he is lined.]' It was of interest whether semantic integration, syntactic integration, or both support the process of co-reference between a pronoun and a subject within sentences about either a person or a thing as an antecedent. For incongruent sentences with person as well as thing-antecedents, we found at clear P600 effect at pronoun position indicating an involvement of syntactic information and/or sentence reanalysis during pronoun processing. The P600 was significantly larger in sentences with a person than a thing as antecedent. This observed difference in P600 effects was interpreted as a possible response to reanalysis of the sentence, including both syntactic and semantic aspects.

For the MEG approach the same basic question arises: Do the processes of establishing coreference between a pronoun and an antecedent differ for person or thing sentences? If so, are they detectable with MEG? Additionally to the ERP-experiment, an event-related fields (ERF) experiment may reveal better spatial information about the investigated processes.

Every current generates magnetic fields according to the right hand rule of physics. The very same principle is applied in the nervous system. Thus, MEG measures the intracellular currents of neurons in the brain giving direct information about the brain's activity, spontaneously or to a given event (Hämäläinen, Hari, Ilmoniemi, Knuntila \& Lounasmaa, 1993; Papanicolaou, 1998). Here, as for ERPs, a large synchronously activated population of 
neurons is needed to generate a measurable signal. Time locking the magnetic signall to an event and averaging several sequences reveals the ERF. MEG provides a better spatial resolution than ERPs because the magnetic permeability of biological tissues is practically the same as empty space. The temporal resolution is comparable to ERP.

Previous MEG : studies on sentence processing focused mainly on the magnetoencephalographic counterparts to the left anterior negativity (LAN) related to early syntactic parsing processing (Friederici, Wang Herrmann, Maess \& Oertel, 2001) or to the N400 related to semantic integration problems (Simos, Basile \& Papanicolaou, 1997; Halgren, Dhond, Christensen, van Petten, Marinkovic, Lewine \& Dale, 2002). So far, there are no published MEG studies related to the later ERP component, the P600 indexing syntactic re-analyses processes.

Friederici and colleagues (2001) investigated the localization of neural generators involved in early syntactic parsing processes in German using MEG by measuring the brain's response to the detection of a phrase structure violation realized as a category error (The fish was caught vs. The fish was * in caught.). Dipole source localization was performed using a realistically shaped standard volume conductor model with PMRI constraints. The results show that the early syntactic parsing processes are supported by activation within the temporal regions, possibly the planum polare, as well as by fronto-lateral regions. These regions are activated bilaterally with dominance in the left hemisphere, which was indicated by dipole strength. The contribution of the left temporal regions to the early syntactic processes seems to be larger than that of the left fronto-lateral regions. The authors concluded that the early syntaxrelated ERP component with its left anterior distribution is not due to a left frontal generator alone, but it seems to originate from both a frontal and a temporall generator.

With respect to pure semantic processing, Simos and colleagues (1997) aimed to localize the sources of the $\mathrm{N} 400$ response elicited in a sentence-reading paradigm. Half of the sentences ended with a semantically appropriate word (e.g. 'He dried his hands with a towel.'), while the other half had inappropriate endings (e.g. "He dried his hands with a hammer."). Evoked. magnetic fields that were recorded over the left hemisphere showed clear magnetic field extrema during the time course of the $\mathrm{N} 400$. The authors reported dipole regions in temporal lobe structures, in the vicinity of the hippocampus and the parahippocampal gyrus (in two subjects) and in posterior temporal regions (in the vicinity of the middle temporal gyrus; in five subjects). The authors stated that these findings are consistent with the view that posterior association cortices in the left hemisphere are involved in word recognition and semantic comprehension during reading. These findings are also supported by a more recent whole 
head MEG study (Halgren et al, 2002). Halgren and colleagues (2002) also investigated sentence endings with semantically congruent or incongruent words. Sentence-terminal incongruent compared to congruent words evoked a large magnetic field over the left hemisphere; peaking at approximately $450 \mathrm{~ms}$ after stimulus onset. Source modeling at this latency with conventional equivalent current dipoles (ECD) placed the $N 400(\mathrm{~m})$ generator in or near the left superior temporal sulcus as has been found by Simos and colleagues (1997). A distributed solution constrained to the cortical surface suggested a sequence of differential activation. Using this method the authors found more activation for incongruent compared to congruent words beginning in planum temporale at approximately $250 \mathrm{~ms}$, spreading to anterior temporal sites (Wernicke's area) at approximately $270 \mathrm{~ms}$, and than by $\sim 300 \mathrm{~ms}$ to left prefrontal areas (Broca"s area). One conclusion from these results was that brain activity that is generated in multiple extended locations may be more realistically modeled using anatomically constrained distributed sources, as opposed to standard ECD.

Based on the reported findings, higher current densities are expected over temporal as well as frontal areas for incongruent pronouns compared to congruent pronouns within the personcondition between $250-400 \mathrm{~ms}$, and possibly later magnetic maxima between $400-600 \mathrm{~ms}$ at similar regions. On the other hand, pure syntactic information processing measured via thingsentences, should reveal increased current densities at temporal and frontal regions predominantly at the left hemisphere between $400-600 \mathrm{~ms}$.

\subsection{Methods}

\section{Subjects and material}

Twenty-one subjects volunteered for paid participation in the experiment. Nine datasets could not be used due to too many artifacts, i.e. moving, eye blinks etc. or technical problems. The final population of subjects comprised twelve native speakers of German, age range 20 to 30 , mean age 23.8 years, 5 women. All subjects had normal or corrected-to-normal vision, were right-handed according to self-report and neurologically healthy.

For material and procedure description, the reader is referred to Chapter 2, Experiment 1 ( $\mathrm{pp}$. $32-33)$. 


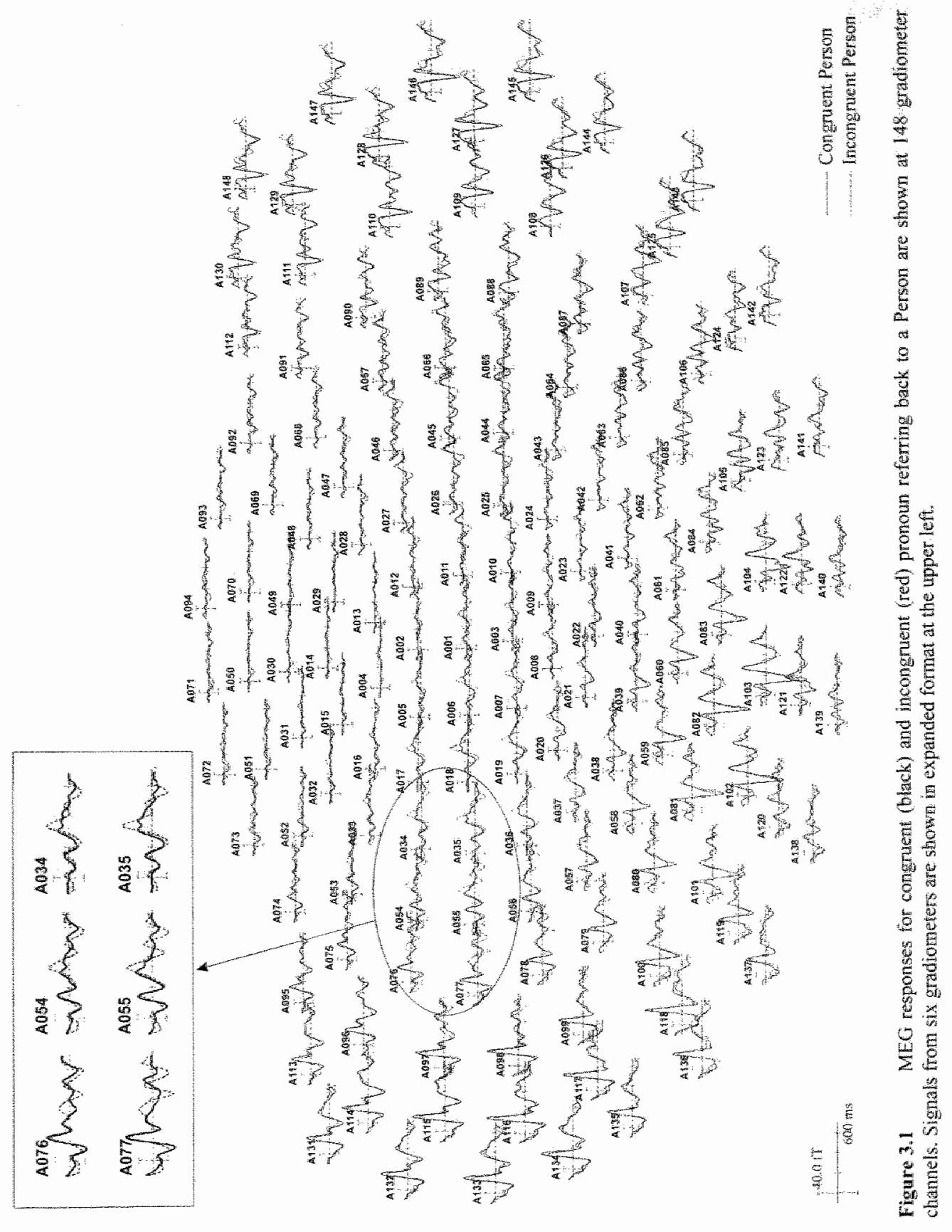




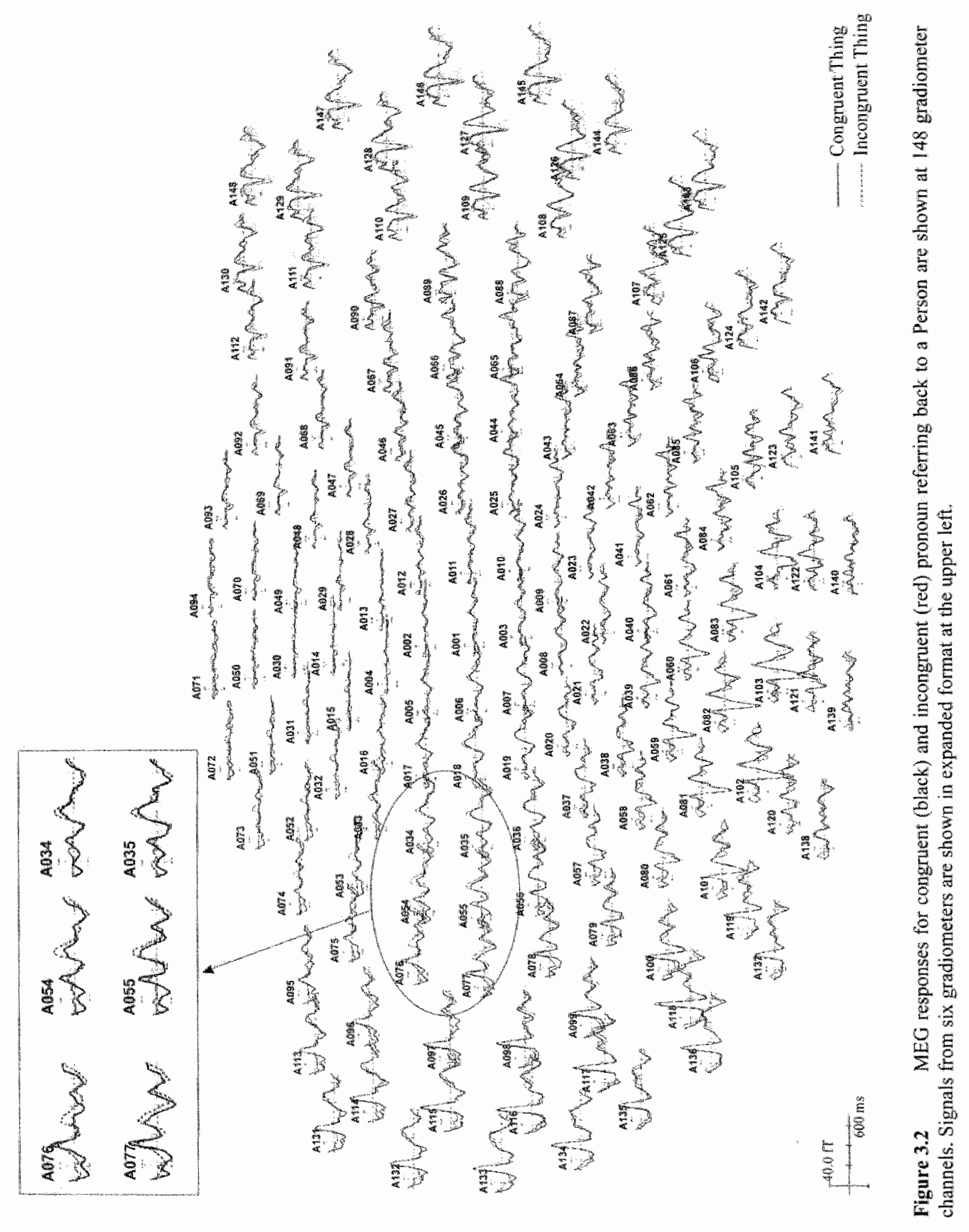




\section{Recording and analysis}

The MEG signals were recorded using a BTi Magnes 2500 whole-head MEG magnetometer system with 148 sensors (Biomagnetic Technologies, Inc.). The MEG signals were filtered with a bandpass of DC $50 \mathrm{~Hz}$ and digitized with a sampling rate of $254 \mathrm{~Hz}$. Additionally, thirty-two surface points on the subject's head were digitized to match the MEG head coordinate system with individual T1 weighted magnetic resonance images using
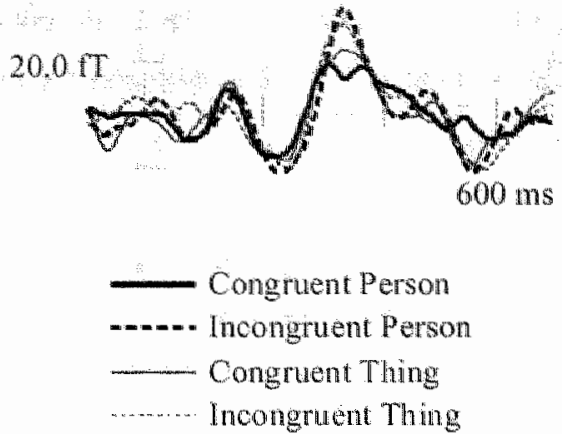

Figure 3.3 MEG responses for all conditions (black: Person; grey: Thing; and solid line: congruent pronoun; dashed line: incongruent pronoun) at gradiometer channel A035 (left temporal position).

BrainVoyager 2000. Spatio-temporal source analysis was performed using the BESA2000 software (MEGIS Software GmbH). The center of the spherical conductor model was set to 5 mm superior and $5 \mathrm{~mm}$ anterior to the posterior commissure based on each subject's MRI. Artifact rejection was performed offline by an automated procedure. Trials including artifacts, such as eye-movements, were rejected from further analysis. By averaging the remaining artifact-free epochs per condition, the event-related fields (ERF) for each condition were derived. The mean number of trials contributing to the average was 83 (person congruent and person incongruent conditions), 86 (thing congruent) and 82 (thing incongruent condition). Then, we calculated difference waves (incongruent minus congruent) as a basis for sources analysis. For source estimation of these difference waves, we then used the mitimum norm estimation (MNE, Hämäläinen et al.s 1993; Dale \& Sereno, 1993) method as a linear estimation technique that does not make explicit assumptions about the number of active sources and their approximate locations and thus objectively models the current sources underlying the magnetic response. The MNE thas been proposed by Tichonow \& Arsenin (1977), and has been further elaborated by Dale \& Sereno (1993). This approach originates from statistical estimation theory. Compared to the traditional ECDs, this method achieves a better solution to the inverse problem of localizing cortical activity in the brain from surface

\section{Person}

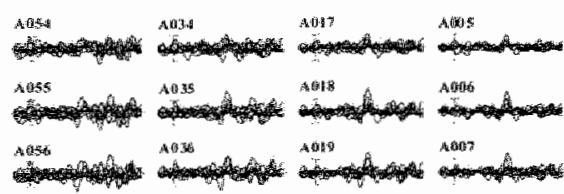

Thing

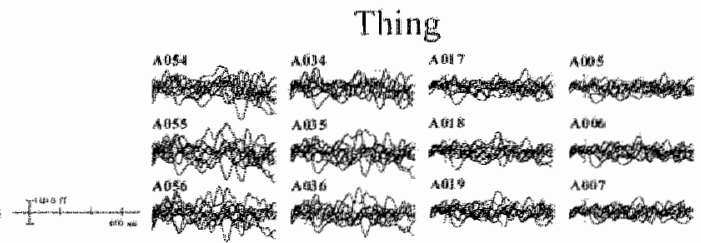

Figure 3.4 Difference waveforms (incongruent numus congruent) are shown for critical pronouns for Person sentences (left panel) and for Thing sentences (right panel) superimposing all subjects. 
recordings by revealing a unique constellation of current elements modeling the recorded magnetic field distribution with the smallest amount of overall current. To find a good estimation on neural generator locations, MNE assumes a priori, among other assumptions that the dipole strength and the noise strength have a certain statistical distribution across the sensors. For example, it seems plausible to assume that the strength of dipoles and the strength of noise co-vary between neighbored sensors (source covariance). The calculated estimation is then compared with the observed data, such that in a linear case, one aims to find the linear operator which minimizes the expected difference between the estimated and the correct solution.

\subsection{Results}

\section{Fields}

Figures 3.1 and 3.2 show the MEG signals to critical pronouns. All conditions reveal an early negative deflection at about $100 \mathrm{~ms}$ followed by a positive deflection peaking at about
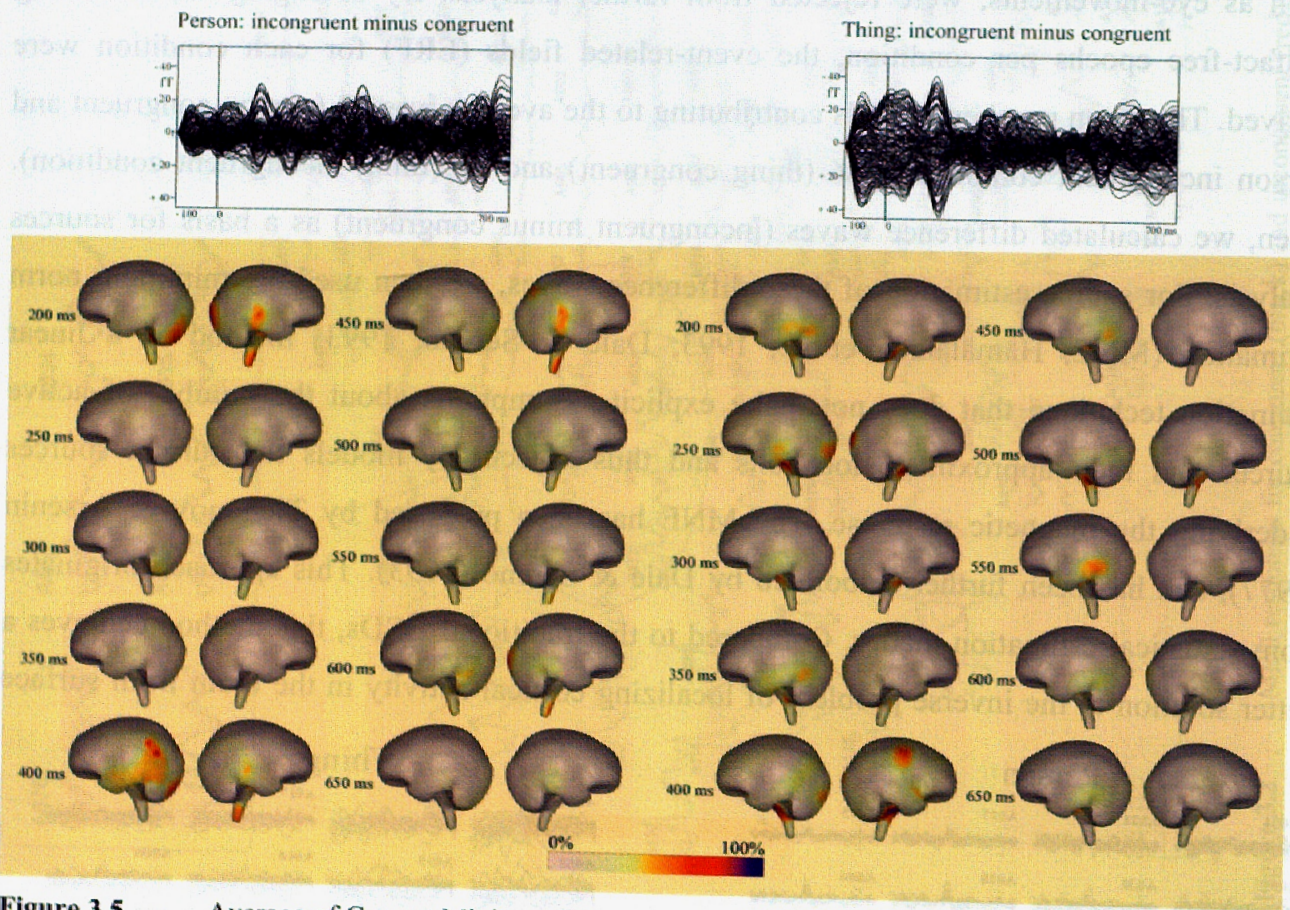

Figure 3.5

Average of Current Minimum Norm Estimates (MNE) for the difference waves of pronouns (incongruent minus congruent) referring back to persons (left panel) or things (right panel) based on a spherical head model cortical activity from 200 to $650 \mathrm{~ms}$ in steps of $50 \mathrm{~ms}$ are shown. On top, one sees the
respective butterfly plot (all channels superimposed) for the difference waves of the ERFs. 
$150 \mathrm{~ms}$. The waves than are identical for all conditions in the time window $150-300 \mathrm{~ms}$ after word onset. Between 300 and 400 ms incongruent and congruent conditions show a difference with incongruent ones being more positive than congruent ones. These holds for both sentence types, especially at sensor sites A076/77, A054/55, A034/35. An overlay of the signal from all condition, displayed in Figure 3.3 at A035 indicates that the overall violation effect is larger for person compared to thing conditions. It also shows that the two congruent conditions differ from each other.

Waveforms were quantified by mean amplitudes in the time-windows from 300 to $400 \mathrm{~ms}$ for the $\mathrm{N} 400 \mathrm{~m}$ and from $400-600 \mathrm{~ms}$ for the electromagnetic counterpart of the P600 (P600m) for a selected set of magnetometers based on visual inspection, namely A005, A006, A007, $\mathrm{A} 017, \mathrm{~A} 018, \mathrm{~A} 019, \mathrm{~A} 020, \mathrm{~A} 034, \mathrm{~A} 035, \mathrm{~A} 036, \mathrm{~A} 054, \mathrm{~A} 055, \mathrm{~A} 056, \mathrm{~A} 076$, and A077. These were subjected to repeated measures analysis of variance that included the Factors Antecedent (Person vs. Thing), Congruency (congruent vs. incongruent) and Magnetometers (15 channels). For the N400m time-window no main effect for Antecedent $(F(1,11)=0.31, p>$ $.55)$, and Congruency $(F(1,11)=0.22, p>.6)$, and no interaction between Antecedent and Congruency $(F(1,11)=0.02, p>.8)$ was found. Also, within the $400-600 \mathrm{~ms}$ time-window no significant effects were found (Antecedent: $F(1,11)=0.31, p>.5$; Congruency: $F(1,11)=.22$, $p>.6$; Antecedent $x$ Congruency: $F(1,11)=0.02, p>.8)$.

Figure 3.4 shows an overlay of all subject's difference waves of person and thing conditions (incongruent minus congruent pronouns) indicating a large variance between subjects. This large variance explains the insignificant results of statistical analysis even though there seemed to be effects visible in Figure 3.1 and 3.2.

\section{Minimum Norm Estimates}

Figure 3.5 shows the calculated Minimum Norm Estimates (MNE) for the difference waves (incongruent minus congruent) of person- (left panel) and thing-antecedents (right panel) from 200 to $650 \mathrm{~ms}$ in $50 \mathrm{~ms}$ steps. The operator solutions, which minimized the difference between estimated and observed solution are plotted in \% match. At an early stage (200 ms), the person conditions show an increase in activity for incongruent compared to congruent pronouns at right parietal sites (Figure 3.5, left panel). Later on (400 ms) there is a left-sided increase of temporo-parietal activation. After that time point, the process of co-reference seems to be finished according to MEG-measurements. For the thing sentences (Figure 3.5, right panel) there is an increase in MNE for incongruent compared to congruent pronouns at about $400 \mathrm{~ms}$ at right parietal areas. Additionally, there seems to be activation within the 
cerebellum and the spinal cond. This aetivation seems to be unreliable out of a structural point of view.

\subsection{Discussion}

The current study was set up to find the electromagnetic counterparts to the ERP effects observed in the first experiment. There, a P600 was found for incongruent compared to congruent pronouns within person- and thing-sentences. This effect was larger for person pronouns compared to thing pronouns. The $\mathrm{P} 600$ is known to be an index for syintactic processing problem, re-analysis, or error detection. Furthermore, in chapter one it was proposed that the $\mathrm{P} 600$ may be influenced by semantic information as well.

The literature concerning $\mathrm{MEG}$ experiments investigating sentence-processing reveals that the $\mathrm{N} 400(\mathrm{~m})$ generator detected via standard ECD localization was found in or near left superior temporal sulcus (Simos et al., 1997; Halgren et al., 2002). Halgren and colleagues additionally investigated a distributed solution and found a more complex network for semantic integration problems, namely beginning in planum temporale at approximately $250 \mathrm{~ms}$, spreading to anterior temporal sites (Wernicke's area) at approximately $270 \mathrm{~ms}$, and than by $\sim 300 \mathrm{~ms}$ to left prefrontal areas (Broca's area). In contrast, investigations concerning the wellknown P600 ERP-component are not published to the author"s best knowledge.

For the present MEG experiment, no reliable differences between incongruent and congruent pronouns were found, neither in person nor in thing sentences in any of the tested timewindows. Nevertheless, we would like to go into the solutions of the MNE as a descriptive approach, taking them as a tendency (ignoring brainstem and cerebellum activation due to regional implausibility). For incongruent relative to congruent pronouns referring back to a person (encountering biological and syntactic gender information; Figure 3.5, left panel) we found that the processing of semantic in combination with syntactic gender violation started early at right temporal sites ( $200 \mathrm{~ms}$ ). This right hemispheric generater is contrary to earlier results lound for early syntactic generators (Friederici et a1., 2001) and semantic integration problems (Halgren et al., 2002), who detected predominantly left hemispheric generators or for one female subject a right asymmetry (Friederici et al., 2001). Due to the weak statistical reliability for the recent study, we should not go into details to this right hemispheric asymmetry for early activation. However, at a later point $(400 \mathrm{~ms})$ we observed a left temporo-parietal activation, followed by a right hemispheric parietal activation. The 
activation of this region is in accordance with results of previous studies of semantic integration problems as revealed by i.e. Halgren and colleagues (2002) or an imaging study using the same experimental material (chapter 4 this volume). In contrast, incongment compared to congruent thing pronouns revealed a different activation pattern (encountering solely syntactic gender information, Figure 3.5 , tight panel): At about $400 \mathrm{~ms}$ a difference activation for thing-conditions (incongruent minus congruent) was found at right parietal sites (Figure 3.5). In comparison to the person-case, processing of thing-pronouns (viz. violation of solely syntactic gender information) starts later on and is located at different areas, namely right parietal sites.

Solely looking at the ERF-results, one could conclude that the experimental pronoun manipulation did not reliably effect syntactic or semantic processing. However, the ERP results reported in the second chapter showed that the given pronoun manipulation is a reliable method to investigate syntactic and semantic gender information processing during pronoun resolution. But then, why is the $\mathrm{MEG}$ method not sensitive to the given manipulation? This may be related to physical characteristics underlying MEG measurements. For example, Eulitz and colleagues stated that the same cognitive process might be differentially imaged by MEG and EEG (Eulitz, Eulitz \& Elbert, 1997). EEG measurements result from the extracellular volume currents triggered mainly by postsynaptic potentials. In contrast, MEG is thought to arise from the intracellular branch of this process, i.e. from currents that flow from the dendrites to the axon terminals. Thereby, MEG is mainly sensitive to currents flowing tangentially (Willamson \& Kaufman, 1990) to the surface of the scalp and to a lesser degree to radial sources (Lütkenhöner, Menninghaus, Steinstrater, Wienbruch, Gißler \& Elbert, 1995). Consequently, EEG and MEG are affected differentially by avernging due to a stronger reduction of "biological noise" for tangential sources than for radial ones. "Biological noise" in this case means that if sources vary across trials and appear in different cerebral regions from trial to trial, their impact on event-related brain responses will be suppressed by averaging. However, this "biological noise" is generally more strongly reduced for tangential sources than for radial ones. Sources in most of the sensory projection areas are primarily tangentially oriented. Consequently, such sources, which are typically activated early in the information processing, have a better signal-to-noise ratio for MEG measurements than for EEG measurement. In cases where the "tangential" noise is strong, and sources are mainly radial, EEG might prove to be the more valuable source of information.

When higher processing stages are the interest of investigation, such as language processing as reported here, the corresponding sources may be widely distributed, and currents flowing 
simultaneously in opposing walls of a sulcus may partially cancel each other out. The remaining equivalent current dipole may have a stronger radial than tangential orientation (Lutzenberger, Elben \& Rockstroh, 1987) and thus will appear with a relatively greater weight in EEG than MEG responses as we could see in the present data set. 


\begin{abstract}
We used event-related functional magnetic resonance imaging (erMRI) to investigate the neural basis of biological and syntactic gender integration during pronoun processing in German. German allows separating both processes experinentally. Overall, syntactic processing actiwted areas adjacent lo Broca" $\$$ area (BA44), whereas processing of the biological sex involved the supramarginal gyrus (BA39) in addition. A comparison with event-related potentials elicited with identical material stggests that the information is integrated 400-700 ms after target onset, visible in both cases as a $\mathrm{P} 600$ with different effect sizes. The results illuminate tamporal and spatial integration of syntactic and semantic processing during the establishment of co-reference between at pronoun and its referring noun.
\end{abstract}




\subsection{Introduction}

Reading emails, newspapers, letters of friends or books is one of our daily businesses. Usually, we are not aware of the details of this cognitive task because it goes so well, mostly automatic. Among other things, in order to understand the message, we have to integrate the meaning and the syntactic relations among single words. For example, in "The woman is happy because she is in love" the pronoun "she" refers back in meaning and syntax to the referent "woman". Thus, pronouns are sophisticated language devices that allow a speaker to refer to an earlier mentioned person or thing. In order to establish this reference, pronouns have to match the conceptual meaning (biological gender), if available, and the syntax of the noun (syntactic gender). In the German language," 'the woman' ('die Frau') for example has a female biological gender with a corresponding feminine syntactic gender. However, 'the jacket' ('die Jacke') has just an (arbitrary) feminine syntactic gender with no semantic gender reflex. Due to this richness of gender properties in German nouns, German pronouns are wellsuited langulage elements to investigate the contribution of semantic and syntactic processing because one can disentangle biological and syntactic gender information expetimentally. We used this dissociation to investigate the involvement of semantic and syntactic information processing to the establishment of co-reference.

How are pronouns integrated into a sentence? Garrod and Sanford (1994) presented a model with two stages for pronoun interpretation (see also Garrod \& Terras, 2000) with (1) a bonding or immediate recovery stage, which is an automatic, more syntax-related process associated with establishing a link between pronoun and a previous expression, and (2) a resolution or immediate integration stage, which involves testing and resolving this link. The integration is responsible for evaluating the established link, i.e. by weighting syntactic and semantic discourse information. In case of congruency, the untegration process finishes with a so-called commitment. In case of incongruency, the integration process fails or is delayed.

So far, pronoun processing has been investigated with reaction time and event-related potentials (ERP). In a preceding ERP-study (Hammer et al., 2005) we performed the identical experiment as in the present $\mathrm{AMRI}$ study with different subjects (for example material see Table 4.1). The conditions revealed a classical P600 pattern, i.e. the ERP signal were more positive during the processing of gender for incongruent compared to congruent pronouns. This P600 effect was larger for person- compared to thing-conditions. The results were interpreted as reflecting the integration of the pronoun into sentence context. We suggested 
that the larger P600 in the person-condition reflects integration of both biological and syntactic gender information, whereas the smaller $P 600$ in the thing-condition reflects syntactic gender information proper.

Because there are no earlier imaging studies on pronoun processing, we would like to review some work engaging syntactic and semantic processing during language comprehension. Indefrey and colleagues (2001) investigated the detection of syntactical errors in meaningless sentences consisting of pseudo words, as in "der Donk der die Feumern lont *telchen tas Grumel. (The [singular] donk who lomes the feumers *telch [plural] the grumel.)" Their data revealed that in syntactic error detection an area of the left dorsolateral prefrontal cortex is specifically involved in syntactic processing aspects of language.

Semantic processing was investigated by Kiehl, Laurens, and Liddle (2002). Subjects read sentences that ended with either a congruent or an incongruent word with previous sentences context. This paradigm is known to elicit an N400 component in event related potential (ERP) research. Example material would be: "The dog caught the bal] in his MOUTH." (congruent). "They called the police to stop the SOUP." (incongruent). The subjects' task was a "makes sense or no-sense" discrimination task related to the last word written in upper case letters. An increase of the Blood oxygen level-dependent (BOLD) response was found in bilateral inferior frontal and inferio-medial temporal cortex, left lateral frontal cortex, left posterior fusiform gyrus, bilateral motor cortex, and supplementary motor area. These findings were discussed as these areas relate to the localization of the cerebral sites underlying semantic processing. In a recent study Hagoort, Hald, Bastiaansen, and Petersson (2004) investigated semantic integration and general world knowledge. Subjects read sentences like: "Dutch trains are yellow/white/sour and very crowded." (Critical words are in italic; true, violation of world-knowledge, semantic violation.) Hagoort and colleagues found that the inferior prefrontal cortex is involved in the integration of both, meaning (semantic) and world knowledge.

Other researchers investigated the processing of syntactic and semantic processing within the same experiment. For example, in a study performed by Newman, Pancheva, Ozawa, Neville, and Ullman (2001) subjects read well-formed sentences randomly intermixed with sentences which either contained violations of syntactic structure ("Yesterday I cut Max's apple with caution." vs. "Yesterday l. cut Max's with apple caution.") or were semantically implausible ("Yesterday I sailled Max's boat to china." vs. "Yesterday I sailed Max's hotel to china."). The authors reported that syntactic violations elicited significantly greater activation than semantic violations primarily in superior frontal cortex. Semantically incongruent sentences 
elicited greater activation than syntactic violations in the left hippocampal and parahippocampal gyri, the bilateral angular gyri, the right middle temporal gyrus, and the left inferior frontal sulcus. These results were interpreted to demonstrate that syntactic and semantic processing result in non-identical patterns of activation, including greater frontal engagement during syntactic processing and larger increases in temporal and temporo-parietal regions during semantic analyses. Röder, Stock, Neville, Bien, and Rösler (2002) used fMRI to investigate the neuroanatomical correlates of semantic and syntactic functions and possible interdependencies between the related brain systems. In a completely crossed design they varied syntactic processing demands (easy vs. difficult to process word order sequences) and the meaningfulness of sentences (real- vs. pseudo-word sentences). Semantic in contrast to pseudo-word sentences elicited a stronger activation in inferior frontal gyrus, and superior and middle temporal gyrus. Syntactic difficulty had its strongest effect within the left inferior frontal region and this effect was more pronounced for semantic than non-semantic speech. The authors interpreted their findings that semantic and syntactic language functions are mediated by partly specialized brain systems but that there nevertheless is a substantial functional overlap of the involved brain structures, especially in left inferior frontal regions. Friederici, Rüschemeyer, Hahne and Fiebach (2003) investigated brain areas supporting the processing of sentence-level semantic and syntactic information. Hemodynamic responses were recorded while subjects listened to correct (i.e. 'Das Hemd wurde gebügelt.' The shirt was ironed.), syntactically incorrect (i.e. 'Die Bluse wurde am* gebügelt.' The blouse was on ironed.), and semantically incorrect sentences (i.e. 'Das Gewitter* wurde gebügelt.' The thunderstorm was ironed.). The authors reported that both anomalous conditions recruited larger portions of the superior temporal region than correct sentences. Processing of syntactic violations specifically involved the anterior portion of the left superior temporal gyrus, the left posterior frontal operculum (adjacent to Broca's area) and the putamen in the left basal ganglia, whereas processing of semantic violations relied primarily on the mid-portion of the superior temporal region bilaterally and the insular cortex bilaterally. Comparing the two incorrect conditions the authors found higher levels of activation for the syntactic compared to semantic condition in the left basal ganglia and for the semantic compared to the syntactic condition in the bilateral mid-portions of the superior temporal gyri. These data indicate that both semantic and syntactic processes are supported by a temporo-frontal network with distinct areas specialized for semantic and syntactic processes. 

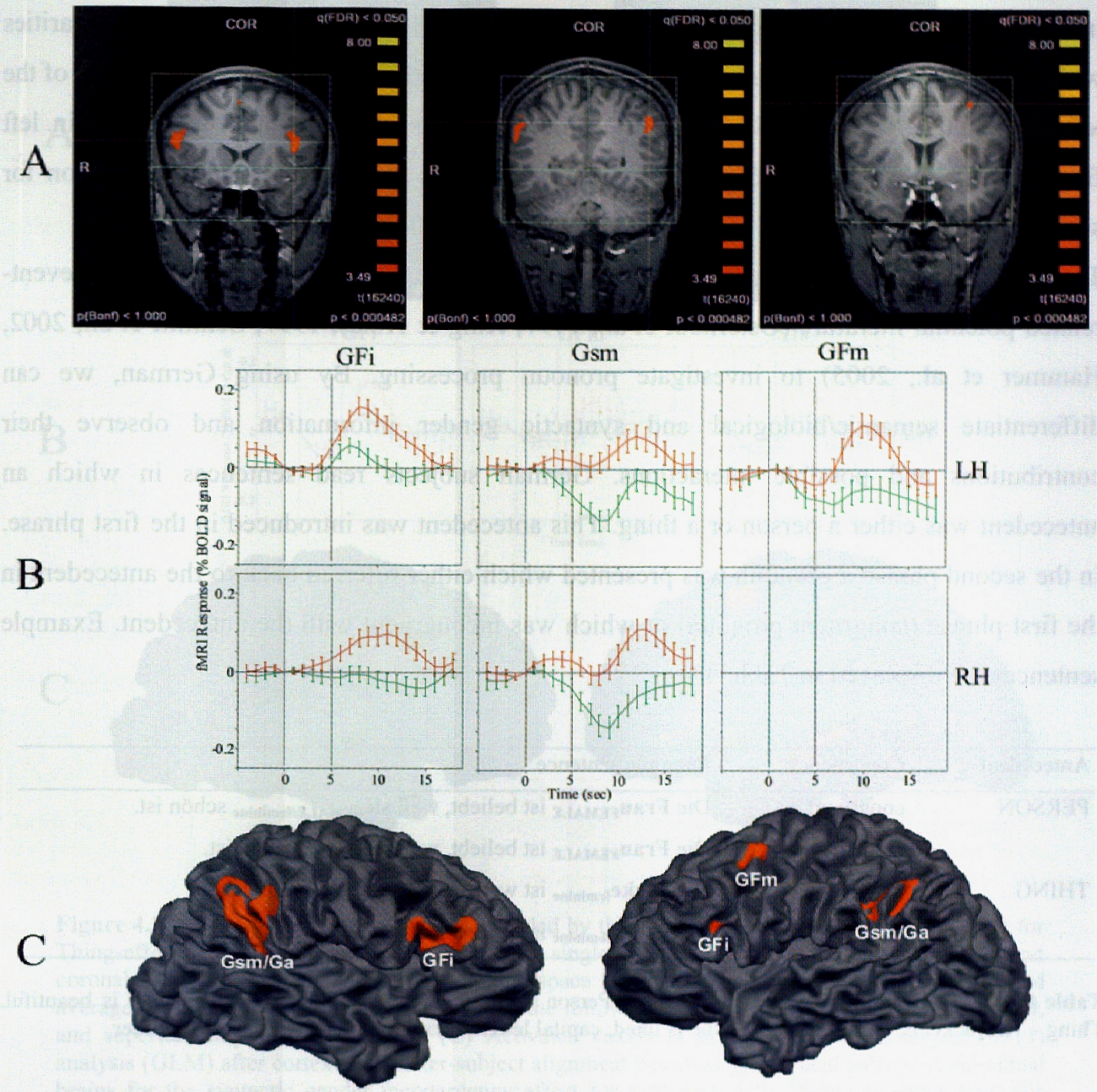

Figure 4.1 (A) Activated voxels as revealed by the across subject $(\mathrm{n}=11)$ analysis (GLM) for Personeffect. Contrast: incongruent minus congruent sentences in this and the following figure. The activations are overlaid onto a single subject's brain (coronal view at Y 3, 17 and -49 ). For detailed Talairach space information see Table 4.2. (B) The middle part in each panel shows the event-related average time course of the BOLD response for the following regions of interest: left $(\mathrm{LH})$ and right $(\mathrm{RH})$ inferior frontal gyrus (GFi), left and right supramarginal/angular gyrus (Gism/Ga), and medial frontal gyrus (GFm). (C) Activated voxels as revealed by across subject $(\mathrm{n}=11)$ analysis (GLM) after cortex-based inter-subject alignment based on gyral/sulcal pattern of individual brains for the biological and syntactic gender incongruency effect for Personsentences. For beta-weights see Figure 4.3, Panel A. 
Taken together, the reported studies reveal an unclear pattern of syntactic and semantic information processing during language comprehension. But still, there are some similarities within these studies. For syntactic processing nearly all studies reported an involvement of the left prefrontal cortex. However, semantic processing was reported to be found within left frontal areas, parietal and temporal regions. Two studies reported a bilateral activation for semantic processing (Kiehl et al., 2002; Newman et al., 2001).

The present erfMRI study uses a violation paradigm, which is well established in the eventrelated potential literature (Osterhout et al., 1997; King \& Kutas, 1997; Schmitt et al., 2002, Hammer et al., 2005) to investigate pronoun processing. By using German, we can differentiate semantic/biological and syntactic gender information and observe their contributions and possible interactions. German subjects read sentences in which an antecedent was either a person or a thing. This antecedent was introduced in the first phrase. In the second phrase a pronoun was presented which either referred back to the antecedent in the first phrase (congruent pronoun) or which was incongruent with the antecedent. Example sentences are displayed in Table 4.1.

\begin{tabular}{|c|c|c|}
\hline Antecedient & Congruency & Example sentence \\
\hline \multirow[t]{2}{*}{ PERSON } & congruent & Die Frau Female ist beliebt, weil sie FEMALE/reminine schön ist. \\
\hline & incongruent & Die Fraufemale ist beliebt, weil $\mathrm{er}_{\text {masculime }}$ schön ist. \\
\hline \multirow[t]{2}{*}{ THING } & congruent & Die Jacke $_{\text {feminine }}$ ist warm, weil sie feminine $_{\text {gefüttert ist. }}$ \\
\hline & incongruent & Die Jacke feminine $_{\text {ist warm, weil }}$ er $_{\text {masculine gefüttert ist. }}$ \\
\hline
\end{tabular}

Table 4.1 Example material. Translation: Person - The woman is popular because she/he is beautiful. Thing - The jacket is warm because she/he is lined; capitall letter = sex, small letter = syntactic gender.

Based on findings reported in the literature reviewed above, we expected an increase of BOLD response in inferior frontal, parietal and temporal regions for incongruent relative to congruent person-sentences (biological and syntactic gender), whereas for incongruent relative to congruent thing-sentences (solely syntactic gender information) we assumed to find activation in inferior frontal regions mainly in the left hemisphere. 


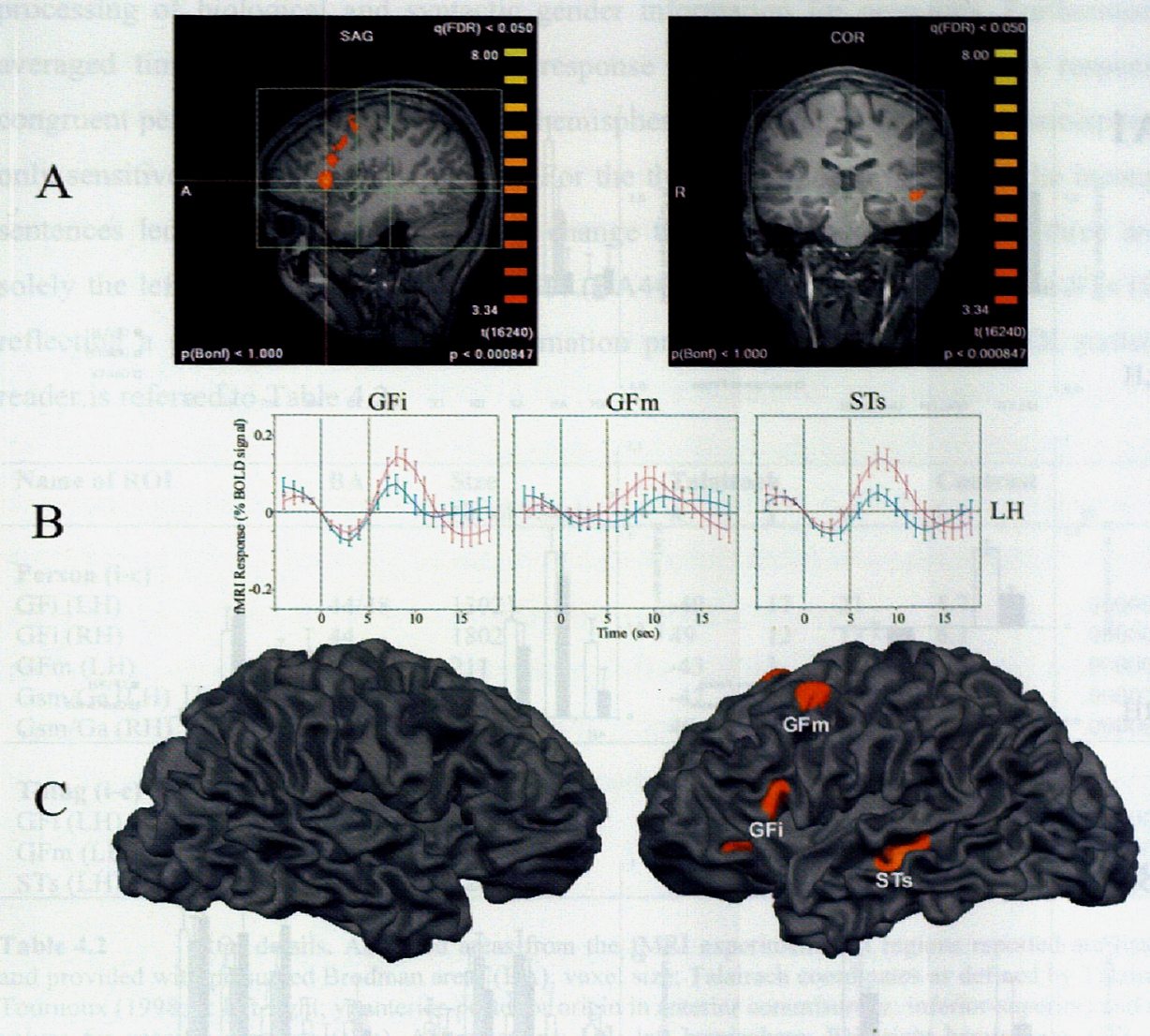

Figure 4.2 (A) Activated voxels as revealed by the across subject (n=11) analysis (GLM) for Thing-effect. The activations are overlaid onto a single subject's brain (sagittal view at $X-40$, and coronal view at $\mathrm{Y}-25$ ). For detailed Talairach space information see Tabie 4.2. (B) Event-related average time course of the BOLD response for the following regions of interest: GFi, LH, GFm LH, and superior temporal sulcus (STs). (C) Activated voxels as revealed by across subject $(\mathrm{n}=11)$ analysis (GLM) after cortex-based inter-subject alignment based on gyral/sulcal pattern of individual brains for the syntactic gender incongruency effect for sentences with Things as antecedent. For beta-weights see Figure 4.3, Panel B. 

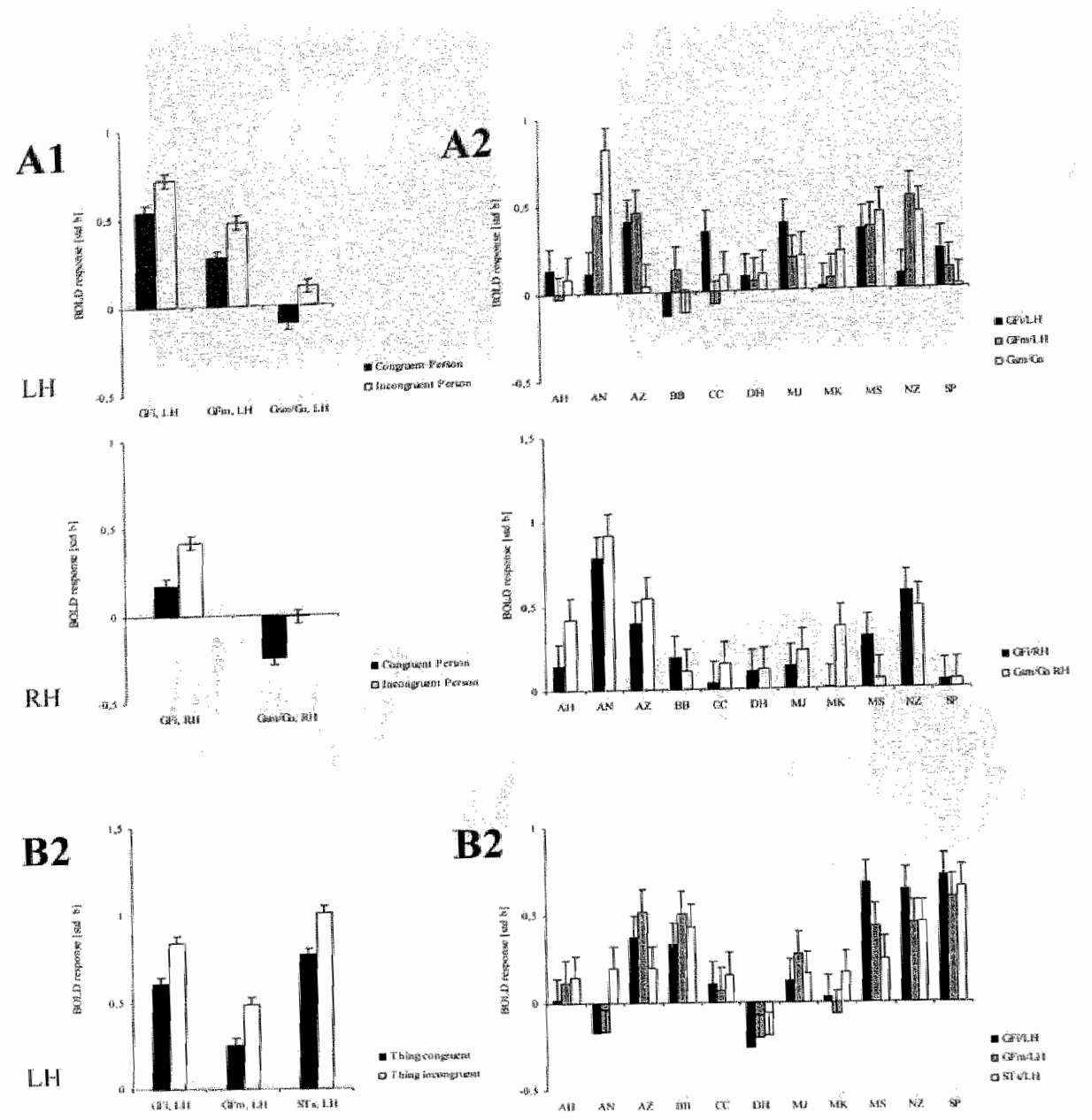

figure 4.3 Responges to congritent and incongruent Person (A) or Thing (B) sentences in regions of interest as reflected in the linear-regression standardized weights (B estimates) averaged ancross subjocts (A.) for person condition, and B) for thing condition). Black bars represent congrvent sentences and white bars represent incongnent sentences. Additionally, the difference (incongnemit minus congruenty of b weights for each individual subject is shown yan pand $A 2$ and B2. Eror bin's indicate the standiud entors of the average b weights.

\subsection{Results}

For the person-condition (Figure 4.1) the incongruent sentences elicited a larger BOLD response change than congruent sentences in two areas on both hemispheres, namely the inferior frontal gyrus (GFi, BA44/48) and the supramarginal/angular gyrus (Gsm/Ga, $\mathrm{BA} 39 / 40$ ) and portions of the lef medial frontal gyrus (GFm, BA6) reflecting a complex 
processing of biological and syntactic gender information for pronouns. Furthermore, the averaged time courses of the BOLD response showed that there was no response for congruent person-sentences on the right hemisphere, indicating that the right hemisphere was only sensitive to incongruent sentences. For the thing-condition (Figure 4.2) the incongruent sentences led to a larger BOLD signal change than congruent sentences in three areas in solely the left hemisphere, namely the GFi (BA44/48), the GFm (BA6) and the STs (BA21) reflecting a pure syntactic gender information processing. For details of ROL statistic the reader is referred to Table 4.2 .

\begin{tabular}{|c|c|c|c|c|c|c|c|}
\hline \multirow[t]{2}{*}{ Name of ROI } & \multirow[t]{2}{*}{ BA } & \multirow{2}{*}{$\begin{array}{l}\text { Size } \\
\text { Nr. of voxel }\end{array}$} & \multicolumn{3}{|c|}{ Talairach } & \multicolumn{2}{|c|}{ Contrast } \\
\hline & & & $\mathrm{x}$ & $y$ & $z$ & $t$ & $\mathrm{p}$ \\
\hline \multicolumn{8}{|l|}{ Person (i-c) } \\
\hline GFi (LH) & $44 / 48$ & 1302 & -40 & 17 & 21 & 5.7 & .0000001 \\
\hline GFi (RH) & 44 & 1802 & 49 & 12 & 17 & 6.3 & .000001 \\
\hline GFm (LH) & 6 & 211 & -43 & 3 & 53 & 4.7 & .000003 \\
\hline Gsm/Ga (LH) & $39 / 40$ & 647 & -47 & -49 & 38 & 4.3 & .00002 \\
\hline Gsm/Ga (RH) & 39 & 3232 & 49 & -58 & 29 & 5.7 & .000001 \\
\hline \multicolumn{8}{|l|}{ Thing (i-c) } \\
\hline GFi (LH) & $44 / 48$ & 4552 & -40 & 22 & 7 & 6.4 & .000001 \\
\hline GFm (LH) & 6 & 536 & -36 & 3 & 52 & 5.3 & .000001 \\
\hline STs (LH) & 21 & 1245 & -47 & -25 & -2 & 6.6 & .000001 \\
\hline
\end{tabular}

Table 4.2 ROI details. Activated areas from the fMRI experiment. All regions reported are listed here and provided with presumed Brodman areas (BA); woxel size; Talainach coordinates as defined by Talairach and Tournoux (1998; $x$ : left-right; $y$ : anterior-posterior origin in anterior commisure; $z$ inferior-superior) and statistic values for specific contrasts ( $t, p)$. Abbreviations: LH: left hemisphere; $R H$ : right hemisphere; $\mathrm{GF}$, inferior frontal gyrus; GFm: medial frontal gyrus; Gsm: supramarginal gyrus; Ga: angular gyrus; STs; superior temporal sulcus.

\subsection{Discussion}

The aim of the study was to disentangle syntactic and biological/semantic gender information processing during pronoun processing as exemplified by the sentences "Die Frau ist beliebt, weil sie/er schön ist." (semantic/syntactic gender information and its violation, incongruent pronoun is in italic font) and "Die Jacke ist warm, weil sie/er gefüttert ist." (syntactic gender information and its violation). Sentences with pronouns referring back to a person revealled an increase of BOLD response for incongruent compared to congruent pronouns in bilateral areas within the inferior frontal gyrus (GFi), and the supramarginal/angular gyrus (Gsm/Ga), and the left medial frontal gyrus (GFm). In contrast, sentences with pronouns referring back to things showed a larger BOLD response for incongruent compared to congruent pronouns in the left GFi/GFm and left superior temporal sulcus (STs). These findings are generally 
consistent with earlier findings for semantic (i.e. Kiehl et al., 2002; Newman et al., 2001), and syntactic processing (i.e. Indefrey et al, 2001; Newman et al, 2001; Röder et al., 2002).

Furthermore, Róder and colleagues reported an interaction of syntactic and semantic mampulation within the left inferior frontal gyrus. The authors suggested that this finding supports the idea that brain areas show some processing specificity, although both language aspects seem to be supported by overlapping brain systems. Our clata support this result as we found an activation of the left GFi for both, Person- and Thing-effect.

It might be fruitful to consider the results from our earlier

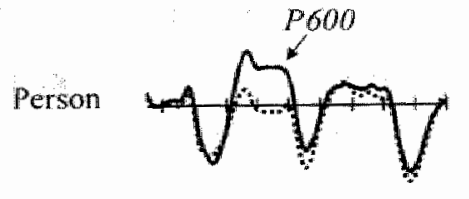
ERP-study (Hammer et al, 2005) with the identical experimental material because ERPs can give insights into the temporal domain of cognitive processes. In general, both violation conditions revealed a $\mathrm{P} 600$ effect within the time-range of 400-700 ms after pronoun onset, with incongruent pronouns being more positive than congruent pronouns suggesting syntactic gender information processing for building up co-reference between pronoun and antecedent. However, this effect was double-sized for the person compared to Thing cases, indicating that the P600 might be a response to reanalysis of the sentence including both syntactic and semantic information processing. Therefore we can conclude that semantic and syntactic integration take place in parallel, i.e. at the same time.

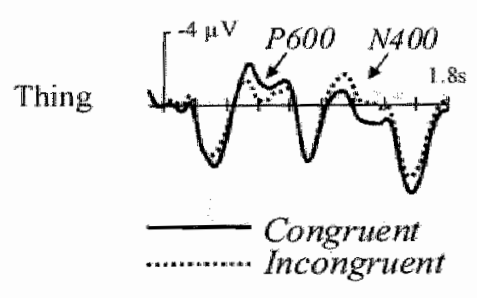

Figure 4.4 Grand average ERPs time locked to the onset of the critical pronoum at electrode $\mathrm{P} 4$ (negativity is up and each hash mark represents $200 \mathrm{~ms}$ of activity, 16

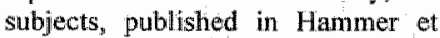
al. 2005). The upper panel shows the ERPS to pronouns in sentences with a person as an antecedent and the lower panel displays the ERPs to pronouns in sentences with a thing as an antecedent. Congruent. pronouns are represented by a solid line and incongruent pronouns by a dashed line.

Together, the ERP and AMRI data show a clear pattern of pronoun processing: in the personcase the language system appears to use both biological and syntactic gender information reflected by an activation of GFi and Gsm/Ga on both hemispheres and the left GFm, seen as a larger BOLD signal change in those areas for incongruent compared to congruent gender sentence types (see Figure 4.1), and in a preceding ERP study using identical material in a large P600 (see Figure 4.4, upper panel). In contrast, for the pure syntactic thing-case, the processing of syntactic violation is reflected by activation of the left GFi, GFm and STs (see Figure 4.2), and as shown in the ERP signal as a comparably small P600 (see Figure 4.4, lower panel). 
How can this observed difference in pronoun processing for person vs thing sentence types be explained? As mentioned in the introduction, Garrod and Sanford (1994, see also Garrod and Terras, 2000) presented a cognitive model with two stages for pronoun interpretation with (1) a low level automatic "bonding" process, and (2) a "resolution" stage. Reading a German sentence with a pronoun the comprehender has to go through (1) syntactic natch in terms of syntactic gender marking and possibly (2) a semantic match in terms of biological gender marking between pronoun and antecedent. We think that our ERP and the erPMRI data present neurophysiological evidence to support this theoretical framework. Pronouns referring to persons can be matched to the antecedent by biological and syntactic information requesting more processing capacity indicated by the large $\mathrm{P} 600$ and the activation of two cortical areas, the bilateral GFi and Gsm/Ga. In contrast, pronouns referring back to things are matched by pure syntactic gender information resulting in a smaller P600 indicating less processing activity and an activation of the left GFi and left STs.

\subsection{Methods}

\section{Subjects}

For the present study we collected $\mathrm{AMRI}$ images from eleven subjects (mean age $28 \pm 5$ years; 4 male, 7 female) while they read the above-described sentences presented in a rapid eventrelated design method (Dale \& Buckner, 1997; Buckner, Bandettini, O'Craven, Savoy, Petersen, Raichle \& Rosen, 1996). All subjects gave their written consent, and were paid for participation.

\section{Material}

One-hundred-twenty sentences, 60 about persons and 60 about things were constructed. Each sentence had two clauses. The first clause in each sentence was the main clause, which described a state of a person or a thing. The person or the thing was the subject of the main clause, and formed the antecedent of the pronoun following later. Care was taken to guarantee equal word frequencies for persons and things using the CELEX-database (Baayen et al., 1995). The second clause was a subordinate clause introduced by the conjunction weil (because). This conjunction was followed by the critical word, a pronoun referring to the person or the thing. All sentences were then copied and the congruent pronoun was replaced 
by a pronoun that was incongnient in terms of biological and syntactic gender in the person condition and by syntactic gender in the thing condition (see Table 1 for examples).

A trial started. with a fxation cross in the middle of the sereen, presented a duration that varied one to five times the Time to Repeat (TR $=1920 \mathrm{~ms}$ ) of image acquisition, and was followed by the word-by-word presentation of the sentence. Each word was presented for 350 $\mathrm{ms}$ with a $250 \mathrm{~ms}$ inter-stimulus interval. Sentence ending words were presented with a period indicating the end of a sentence.

\section{Functional measirement}

We measured 24 transversal slices at 3 Tesla (Magnetom Trio, Siemens) using an echoplanar-imaging sequence. The pulse-sequence parameters were as follows: plane resolution, $3,5 \times 3,5 \times 3,5 \mathrm{~mm}$; gap, 1,2; field of view (FOV), $224 \mathrm{~mm}$, acquisition matrix $64 \times 64$; time to repeat (TR), $1920 \mathrm{~ms}$; time to echo (TE), $35 \mathrm{~ms}$; flip angle (FA), $75^{\circ}$. One functional run consisted of 375 volumes. In order to avoid a $\mathrm{T} 1$ saturation effect we did not present any material during the first 10 volumes and excluded the first four volumes from further analyses.

\section{Anatomical measurements}

Each subject underwent a high resolution T1-weighted anatomical scan at 3 Tesla (Magnetom Trio, Siemens) using a three-dimensional T1-fast-low-angle shot sequence lasting $9 \mathrm{~min}$ and

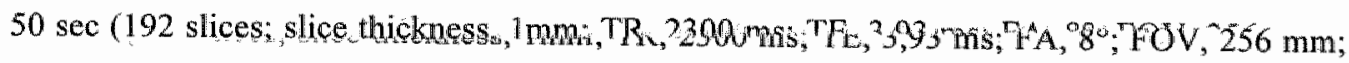
matrix. 256×256).

\section{Arralysis}

Data analysis included preprocessing (3D motion correction, spatial smoothing and temporal filtering $)$ coregistration, Talairach transformation of scans, volume and surface rendering, and cortex reconstruction using BRAINVOYAGER 2000 and BRAINVOYAGER QX software. For multiple regression analysis of the functional data, a general linear model (GLM) with predictors for each experimentall condition was computed. Using contrast ( $t$ ) maps, significant differences between conditions were assessed. Multiple comparisons were controlled by the False Discovery Rate (FDR) approach at a level of $q=0.05$ (Genovese, Lazar \& Nichols, 2002). Reported activations are based on fixed effects group statistics.

In order to increase the sensitivity of statistical analysis, an improved alignment of the brains was achieved by a cortical inter-subject alignment procedure. A polygon mesh of each 
cortical hemisphere was obtained by segmenting and tessellating the white/grey matter boundary (Dale \& Sereno, 1993; Fischl, Sereno \& Dale, 1999, Kriegeskorte \& Goebel, 2001 ). Then, cortical time-courses were created by sampling the functional time-series at positions corresponding to the nodes of the cortical mesh. Polygon meshes (and corresponding cortical time-courses) were morphed into alignment using spherical representations of the cortical sheet (Fischl, Sereno, Tootell \& Dale, 1999). For thresholding the equivalent t-values to the FDR analysis were chosen ( $\mathrm{T}>3.5)$. 


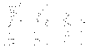

से 


\title{
Interaction of semantic and syntactic information and working memory during pronoun resolution: an ERP study
}

\begin{abstract}
Personal pronouns are used to refer back to an earlier mentioned person or thing. In German, this co-reference can be based on syntactic and/or semantic gender information. The aim of this study was to investigate the interaction of demands on verbal working memory (VWM) and syntactic and semantic information during the process of building up co-reference between a pronoun and its antecedent. Healthy young subjects were subjected to a word by word reading paradigm in conjunction with the reconding of event-related potentials (ERP). We experimentally disentangled syntactic and biological/conceptual gender information by using German sentences either about a person (biological and syntactic gender assigmment) or about a thing (syntactic gender assignment) followed by a pronoun congruent or incongruent in terms of gender. Additionally we manipulated VWM by increasing the distance across noun phrases between pronoun and antecedent resulting in a short distance (SD) and a long distance (LD) VW/M condition. In general this VWM manipulation affected the ERP N400/P600 patterns of our sentence types indicating that VWM interacts with semantic and synthotic processing. For sentences with persons as antecedent in $\mathrm{SD}$ conditions we found an N400 effect for incongruent compared to congruent pronouns, indicating involvement of semantic integration. In contrast, for LD conditions we abserved a $\mathrm{P} 600$ but no N400 effect indicating the involvement of syntactic reanalysis without independent semantic integration. Since this effect was larger for LD person compared to SD thing conditions, we interpreted this finding as reflecting increased processing difficulty due to temporal distance and double gender wiotation (biological/syntactic). For sentences with things as antecedent, within $\mathrm{SD}$ conditions we found a $\mathrm{P} 600 \mathrm{effect}$ indicating an involvement of syntactic reanalysis, whereas $L D$ conditions revealed no effect indicating no different processing of incongruent and congruent pronouns in that case. In addition; we found an N400 on the word following the pronoun for $\mathrm{SD}$ and $\mathrm{LD}$ thing sentences, indicating an ongoing search process for $i . \mathrm{b}^{\mathrm{s}}$ an actor outside sentence context independent of distance manipulation. In general, our diferent N400/P600 patterns on pronoun position suggest that a parser switches preference between semantic and syntactic information access during pronoun processing depending on VWM and information awailability.
\end{abstract}




\subsection{Introduction}

Personal pronouns are linguistic devices which allow us to refer back to an earlier mentioned person or thing in the discourse and thus creating coherence in written or spoken language. Considering the sentence "The woman is smiling because she is happy." it is easy to tell that she belongs to the woman in this sentence. Several cognitive models outline the details of this process (Garrod \& Sanford, 1994; Graesser, Millis \& Zwaan, 1997; Gordon \& Hendrick, 1998): Garrod and Sanford (1994) presented a model with two stages for pronoun interpretation (see also Garrod \& Terras, 2000) with (1) a bonding or immediate recovery stage, which is an automatic, more syntax-related process associated with establishing a link between anaphor and a previous expression, and (2) a resolution or immediate integration stage, which involves testing and resolving this link. The integration stage is responsible for evaluating the established link, i.e. by weighting syntactic and semantic discourse information. In case of congruency, the integration process finishes with a so-called commitment. In case of incongruency, the integration process fails or is delayed.

But how does the reader's brain link a pronoun to the antecedent? Event-related potentials (ERP) are a suitable method to investigate cognitive processes especially in the temporal domain. ERP research of the last decades has shown that semantic integration problems elicit a negative deflection with a peak around $400 \mathrm{~ms}$ and a right central-parietal negative maximum for incongruent compared to semantically congruent words, the so-called N400 component (Kutas \& Hillyard, 1980,1983 ). Problems with syntactic integration become manifest in a positive shift that starts at about 400 to $500 \mathrm{~ms}$ after word onset, the so-called P600/SPS effect (Osterhout \& Holcomb, 1992; Hagoort et al., 1993). In addition, it has been shown that syntactic processing problems can give rise to an earlier left anterior negativity (LAN) (e.g. Kluender \& Kutas, 1993; Friederici, Hahne \& Mecklinger, 1996). These frontal negative ERP effects in general are observed under conditions of increased working memory load in sentence (Kluender \& Kutas, 1993; Carpenter, Miyake \& Just, 1995; Minte et al., 1998b; Streb et al., 1999; Fiebach et al., 2001) as well as discourse processing (Graesser et al., 1997; van Berkum et al., 2003). Negativities with a similar distribution have also been observed for syntactic violations (Friederici et al., 1996; Münte et al. 1993).

ERP' studies on pronoun processing have shown that a disagreenent in syntactic gender information between pronoun and antecedent reveals a P600/SPS compared to congruent gender information indicating an involvement of syntactic gender information processing 
(Osterhout \& Mobley, 1995; Schmit et al, 2002, Hammer et al, 2005). On the other hand, a disagreement in biological/conceptual gender reveals an N400 (King \& Kutas, 1997), and a disagreement in biological and syntactic gender reveals either an N400/P600 complex. (Schmitt et al, 2002) or a P600 which is twice the size of a syntactic gender violation condition (Hammer et al, 2005).

In order to be able to link an incoming pronoun to a potential antecedent, possible antecedents are supposed to be re-activated (Nicol \& Swinney, 1989; MacDonald \& MacWhimney, 1990). There is some ERP evidence that working memory is involved in the process of pronoun resolution. For example Streb and colleagues (1999) investigated the effects of pronoun and proper name anaphors in both parallel (easy) and non-parallel (difficult) syntactic discourse structures. A parallel structure means that pronoun and noun have the same syntactic function; and the establishment of the co-reference is relatively easy (such as in: "Peter visits Julia in the hospital. There he asked a question to the physician."). In contrast, in a non-parallel structure pronoun and antecedent have a different syntactic function, which is usually more difficult to interpret (such as in: "Peter visits Julia in the hospital. There she asked a question to the physician."). Streb and colleagues assumed that the nonparallel conditions involve more working memory load due to the higher degree of syntactic processing difficulty. They reported two ERP results: (1) Pronoun anaphors evoked a more pronounced negativity than proper name anaphors between 270 and $420 \mathrm{~ms}$ latency range over the frontal cortex electrodes. Another relative negativity occurred between 510 and $600 \mathrm{~ms}$ over the parietal sites. (2) Anaphors in nonparallel positions compared to parallel positions were more negative over parietal sites. The authors suggested that these two effects are functionally related to discourse resolution including a possible search in working memory for the suitable antecedent.

The aim of the present study was to investigate whether semantic integration, syntactic integration, or both interact with verbal working memory during establishing co-reference between a pronoun and the corresponding antecedent in sentences. In one condition a person was introduced as an antecedent and later referred to by a pronoun, which either agreed in biological/syntactic gender or not (biological/syntactic gender violation). In a second condition, a thing was introduced as an antecedent and the corresponding pronoun either agreed in syntactic gender with the antecedent or not (syntactic gender violation). To manipulate verbal working memory in pronoun processing the distance between antecedent and pronoun was either short (short distance $=\mathrm{SD}$ condition) or long (long distance $=\mathrm{LD}$ condition), see material in Table 5.1. This manipulation is in line with standard settings for 
VWM manipulations (Daneman \& Carpenter, 1980; King \& Kutas, 1997). Increasing the distance between the pronoun and the antecedent demands more VWM capacity because possible antecedents have to be re-activated for further processing (Nicol \& Swinney, 1989; van Berkum et al., 2003). While subjects read these eight different types of sentences their event-related potentials (ERP) were recorded. Following the logic of earlier ERP-research, we propose the following hypothesis: according to a strictly modular view, syntactic and semantic as well as working memory could work independently of each other. Following this, we expect an N400/P600 complex for double violations in the person sentence types, a P600 for syntactic violation in the thing sentence types, and we expect to find a LAN component for incongruent compared to congruent pronouns that is larger within the long distance conditions. Alternatively, according to a more interactive view, working memory could indirectly influence semantic and syntactic processing. In this case, we would expect a difference of the semantic and syntactic effect size of the N400 and/or P600 in the SD compared to the LD conditions:

\section{Condition Sentence}

SD P: Person as antecedent in short distance

C. congruent Der Huptlingmalifnas ist kriegerisch, weill er Malkwas gewinnen will.

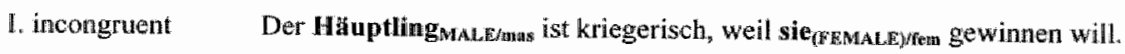

SD T: Thing as antecedent in short distance

C. congruent Der Apfel mus ist süB, weil er (MALE)/mas reif ist.

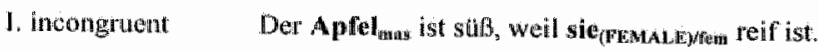

LD P: Person as antecedent in long distance

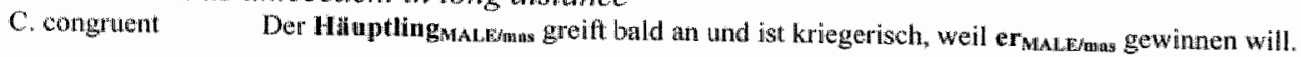

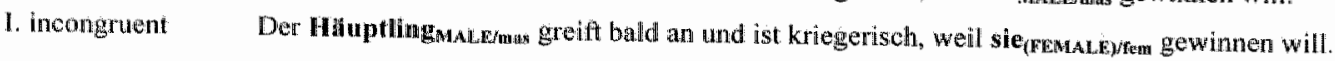

LD T: Thing as antecedent in long distance

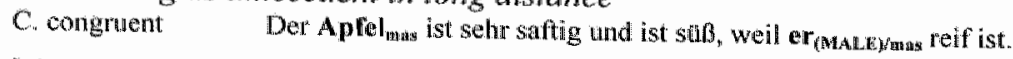

1. incongruent Der Apfel ${ }_{\text {anus }}$ ist sehr saftig und ist süß, weil sie [FEMALE)/fen $_{\text {reif }}$ ist. Tuble 5.I
wants to win. SD T: The apple is sweet because he/she (it) is ripe. LD P. The chief attacks Soon and is martial
because. he/she wants to wing FEMALEMALE - biological genden, mas = apple is very juicy and is sweet because he/she (it) is ripe. FEMALE/MALE = biological gender, mas = syntactic gender masculine, fem = syntactic gender feminine. 


\section{Short Distance}
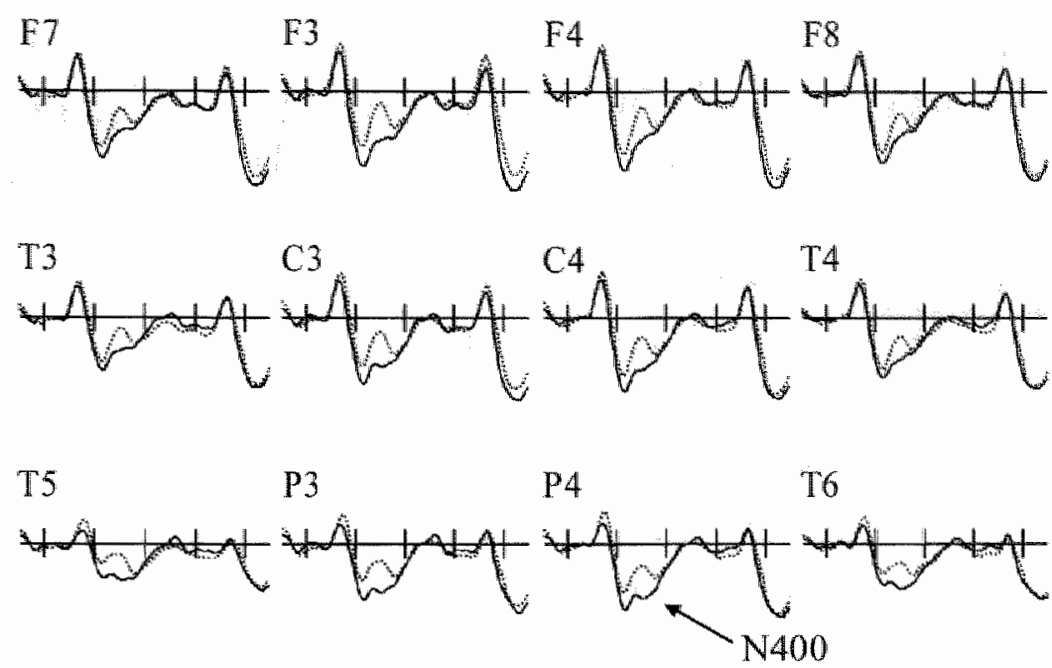

\section{Long Distance}
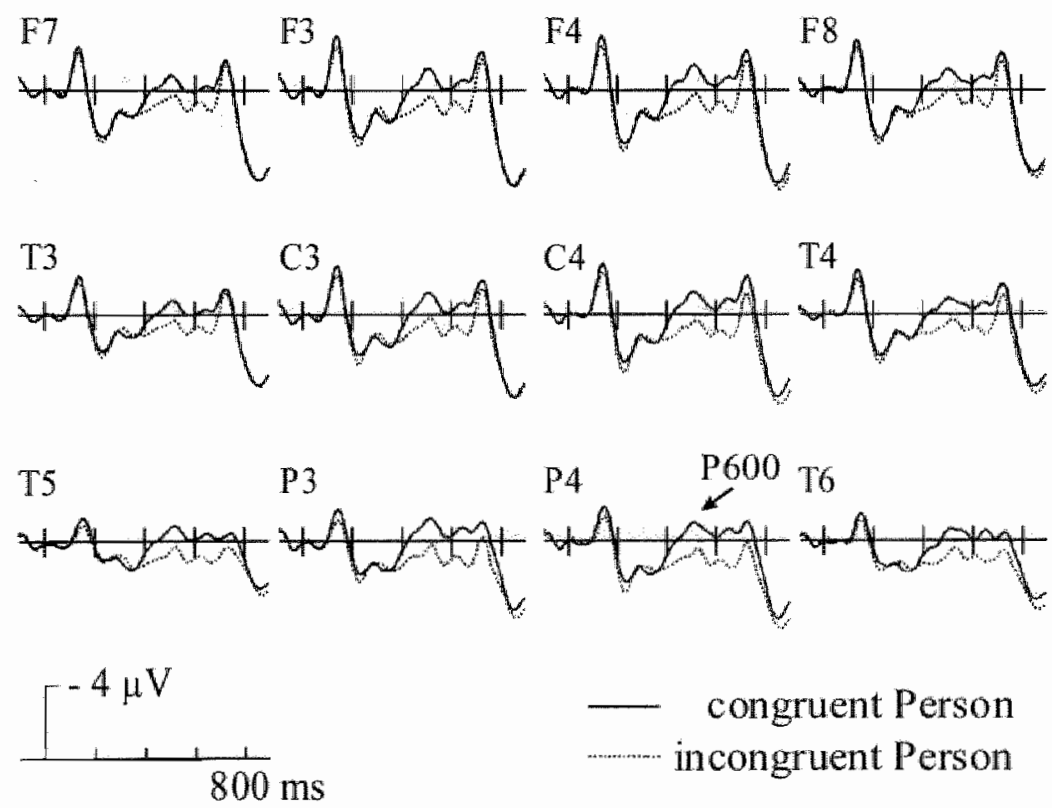

Figure 5.1 Grand average ERPs time locked to the onset of the Person-pronouns in SD sentences (upper panel) and LD sentences (lower panel) at a selected set of electrodes (negativity is up and each hash nark represents $200 \mathrm{~ms}$ of activity in this and in the following figures). 
Short Distance
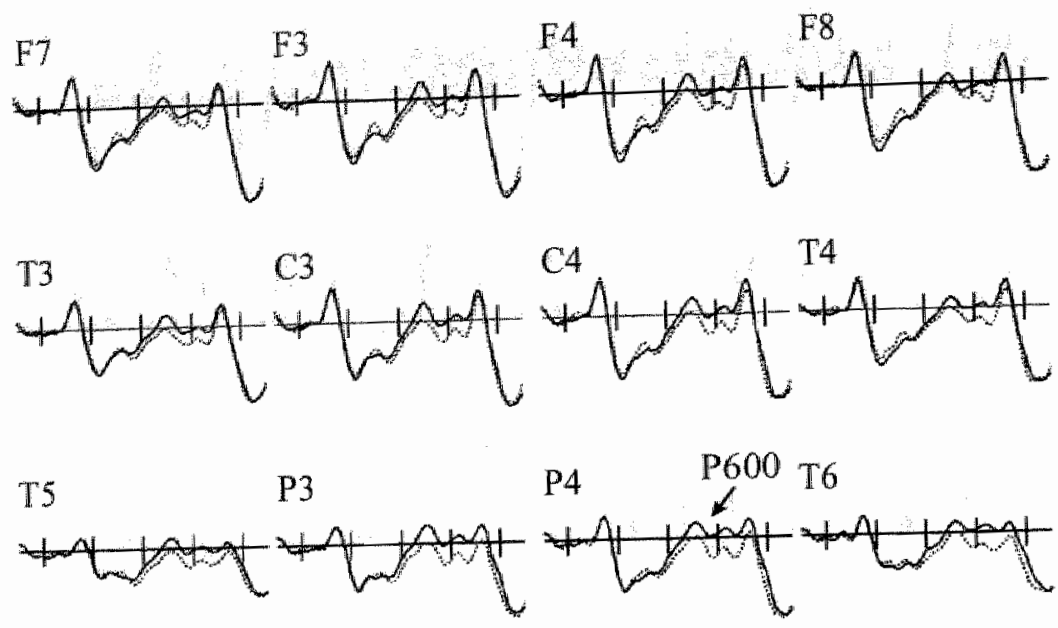

\section{Long Distance}
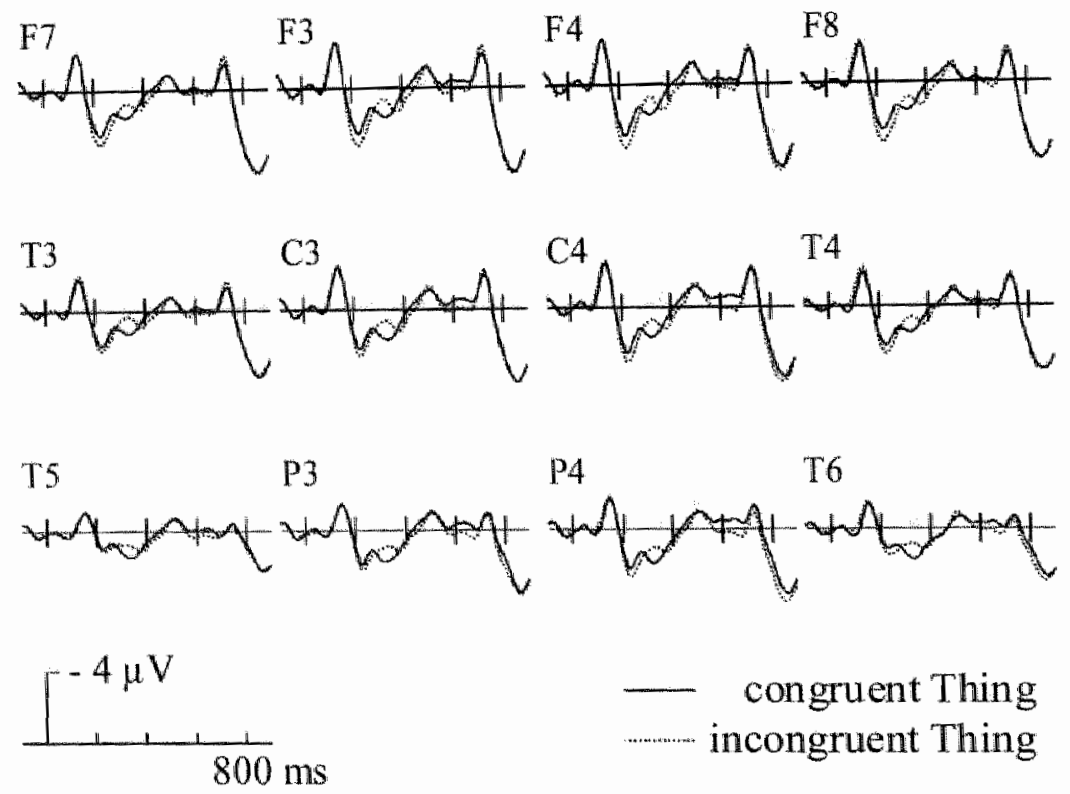

Figure 5.2 Grand average ERPS time locked to the onset of the Thing-pronouns WM Sentences (upper panel) and LD sentences (lower paneil) at a selected set of electrodes. 


\subsection{Methods}

\section{Parricipants}

Twenty German native speakers from the student population of the Maastricht University volunteered to take part in the experiment with written consent and were paid for their participation. All had normal or corrected-to-normal vision, were right-handed and neurologically healthy. Four datasets were not included in later analysis because of too many artifacts or technical problems, leaving data of sixteen participants (12 women, mean age $22 \pm$ 2.2 years).

\section{Material and design}

For half of the material we used sentences from an earlier study (Hammer et al., 2005): Twohundred-forty sentences, 120 about persons and 120 about things, had two clauses each. The first main clause described a state of a person or a thing. The person or the thing was the subject of the main clause, and formed the antecedent of the pronoun following later. Care was taken to guarantee equal word frequencies for persons and things using the CELEXdatabase (Baayen et al., 1995). The second clause was a subordinate clause introduced by the conjunction weil (because). This conjunction was followed by the critical word, a pronoun referring to the person or the thing. All sentences were then copied and the congruent pronoun was replaced by an incongruent pronoun. These sentences comprised the SD conditions of the current experiment. To manipulate verbal working memory (VWM) in pronoun processing the distance between antecedent and pronoun was increased by adding four words after the antecedent in the same sentences resulting in a total of 480 different sentences (see Table 5.1). Altogether this resulted in a $2 \times 2 \times 2$ design with VWM (SD/LD), antecedent type (person/thing $\mathrm{P} / \mathrm{T}$ ), and congruency (congruent/incongruent $\mathrm{C} / \mathrm{I}$ ) as factors. To minimize repetition the sentences were distributed across two different lists, counterbalancing antecedent type, congruency, and VWM. The sentences of each list were then pseudorandomized over four blocks, in such a way that repetitions of antecedents were kept as far apart as possible. We measured eight subjects per list, which were pooled again for analysis. To ensure reading and sentence processing content related question were presented after 10 t 5 sentences. Subjects were asked to answer the questions with "yes' or "no" by pushing one of two mouse buttons. This task was independent of the pronoun manipulation in order to avoid interference of the explicit task performance with the implicit electrophysiological measures. Only subjects with at least $80 \%$ correct answers in the comprehension task were accepted. 
The words were presented white on black background in a 16-point font size (Arial) at a viewing distance of about $100 \mathrm{~cm}$ (subtending approximately 0.5 by 1.2 degrees of visual angle for critical words).

\section{Procedure}

Subjects were tested in a dimly lit sound-attenuating room while sitting in a comfortable chair. Subjects were asked to read the sentences concentrated and carefully because they were supposed to answer questions concerning the content of the sentences. They should fixate on the screen and avoid possible movements during the word-by-word presentation of the sentences. Between sentences and during the question subjects were free to blink and move their eyes.

A trial started with a fixation cross in the middle of the screen (duration $2050 \mathrm{~ms}$ ) which was followed by the word-by-word presentation of the sentence. Each word was presented for 350 $\mathrm{ms}$ with a $250 \mathrm{~ms}$ inter-stimulus interval. Sentence ending words were presented with a dot indicating the end of a sentence, followed by a blank screen for $850 \mathrm{~ms}$.

Questions were presented on the screen for $4 \mathrm{~s}$. One block lasted approximately 20 minutes. The entire experiment, including instructions, electrode application, and removal took about 2.5 hours.

\section{EEG recording and analysis}

Electroencephalography (EEG) signals were registered with a digitization rate of $250 \mathrm{~Hz}$ and filtered with a band pass of $0.01-50 \mathrm{~Hz}$. Twenty-nine tin electrodes mounted in an elastic cap were positioned according to the $10 / 20$ system $(\mathrm{Fz}, \mathrm{Fcz}, \mathrm{Alz}, \mathrm{Cz}, \mathrm{Cpz}, \mathrm{Pz}, \mathrm{Oz}, \mathrm{F} 7 / 8, \mathrm{~F} 3 / 4$, $\mathrm{C} 3 / 4, \mathrm{~T} 3 / 4, \mathrm{~T} 5 / 6, \mathrm{P} 3 / 4, \mathrm{O} / 12, \mathrm{Fpl} / 2, \mathrm{Fc} 3 / 4, \mathrm{Tp} 7 / 8, \mathrm{Cp} 3 / 4$, Bio-signals were recorded with $a$ loft mastoid reference, and were offline re-referenced to the mean of the activity at the two mastoids. Blinks and vertical eye-movements were recorded with electrodes placed at the suband supraorbital ridge of the left eye. Lateral eye-movements were monitored by a bipolar montage using two electrodes placed on the right and left external canthus. Eye movements were recorded in order to allow for later offline rejection. All electrode impedances (EEG and EOG) were kept below $5 \mathrm{k} \Omega$.

From the continuous signal epochs were created of $1024 \mathrm{~ms}$ starting $100 \mathrm{~ms}$ prior to pronoun onset. These epochs were monitored for artifacts, such as eye-movements, by an automated procedure. Trials including artifacts were rejected from further analysis (10\% rejection on average, no difference between conditions). By averaging the remaining artifact-free epochs 
per condition the Event-related potentials (ERPs) were derived. Waveforms were quantified by mean amplitude measures (relative to a $100 \mathrm{~ms}$ pre-stimulus baseline) to assess LAN/N400 (time window 200 to $400 \mathrm{~ms}$ ) and P600 (400 to $700 \mathrm{~ms}$ ) components. These windows were derived from previous pronoun experiments of our group and corroborated by wisual inspection of the grand average ERPs. These measures were subjected to repeated measures analysis of variance that crossed the factors VWM (2; short vs. long, SL), Antecedent type (2; person vs. thing, PT), Congruency (2; congruent vs. incongruent, CI) and 'Electrode sites' (29 levels). Additionally, we compared the mean amplitudes of conditions in both time-windows by separate analyses for Congruency (SPC vs. SPI, LPC vs. LPI, and STC vs. STI, LTC vs. LTI) and VWM (SPC vs. LPC, SPI vs. LPI, and STC vs. LTC, STI vs. LTI) in order to trace back possible effects. Finally, ERPs were averaged for $2048 \mathrm{~ms}$ epochs with a 100-ms prestimulus interval in order to analyze the processing of the word following the pronoun. These measures were subjected to repeated analysis of variance following the same logic as above and an additional analysis for the frontal $(\mathrm{F} 3, \mathrm{Fz}, \mathrm{F} 4)$, central $(\mathrm{C} 3, \mathrm{Cz}, \mathrm{C} 4)$ and parietal line $(\mathrm{P} 3, \mathrm{Pz}, \mathbb{P} 4)$ in a time window from 1000 to $1300 \mathrm{~ms}$ (corresponding to a $400-700 \mathrm{~ms}$ timewindow for the word after the pronoun). The Greenhouse-Geisser correction for inhomogeneity of covariance was applied whenever an evaluated effect had more than one degree of freedom in the numerator.

\subsection{Results}

The grand average ERPs time-locked to the onset of the pronouns are shown in Figure 5.1 and 5.2 (comparing congruent and incongruent pronouns). Figure 5.1 shows the ERPs of pronouns referring to the person-antecedent within the SD conditions (upper panel) and within the $L D$ conditions (lower panel). The ERPs of pronouns referring back to the thing antecedent can be seen in Figure 5.2 within SD condition (upper panel) and within LD condition (lower panel). All eight conditions elicit a N1-P2 complex, which is typical for visually presented material. In the SD-person condition, incongruent compared to congruent pronouns are characterized by a widespread negativity between 200 and $400 \mathrm{~ms}$ after pronoun onset. Past $400 \mathrm{~ms}$ the waveforms to incongruent and congruent pronouns are highly similar in the SD-person condition. 


\begin{tabular}{|c|c|c|c|}
\hline Source & dif & $F$ & $P(G O)$ \\
\hline \multicolumn{4}{|l|}{ OmilWuS ANOVA (29 electrodes) } \\
\hline VWM (chort wis. long) & 1,15 & 5.58 & .03 \\
\hline VWM X delectrode & 28,420 & 291 & .03 \\
\hline WWM Congrucncy $x$ Electrode & 28,420 & 2.42 & .04 \\
\hline \multicolumn{4}{|l|}{ Separate ANOVAs for Congruency (29 electrodes): } \\
\hline SD P: Congruengy (congruent ws incongruent) & 1.15 & 6.40 & .02 \\
\hline Congruency $\times \mathrm{E}$ & 28,420 & 3.96 & .002 \\
\hline SD $\mathbf{T}$ : Congruency (congruent ws incongruent) & 1,15 & 0.09 & \\
\hline Congruency $\times \mathrm{E}$ & 28,420 & 0.88 & \\
\hline LD P: Congruency (congruent ws. incongruent) & 1,15 & 0.20 & \\
\hline Congruency $\times \mathrm{E}$ & 28,420 & 0.49 & \\
\hline LD T: Congrutercy (congruent vs. incongruent) & 1,15 & 0.02 & \\
\hline Congruency $x \mathrm{El}$ & 28,420 & 0.87 & \\
\hline \multicolumn{4}{|c|}{ Sepstrate ANOVAs for Working Memory (29 electrodes): } \\
\hline CP: VWM (short ws. long) & 1,15 & 9.52 & .008 \\
\hline Mem x Eil & 28,420 & 3.25 & .004 \\
\hline IP: VWM (short vs. long) & 1,15 & 0.01 & \\
\hline VWM $\times \mathrm{EI}$ & 28,420 & 1.46 & \\
\hline Cr: VWM (short vs. long) & 1,15 & 2,06 & \\
\hline $\mathrm{VWM} \times \mathrm{EI}$ & 28,420 & 1.93 & .08 \\
\hline IT": VWM (short vs. long) & 1,15 & 2.28 & \\
\hline $\mathrm{VWM} \times \mathrm{GI}$ & 28,420 & 0.56 & \\
\hline
\end{tabular}

Table 5.2 Mean ERP amplitude ANOVAs in the 200-400 ms Latency Range. WWM, verbal working

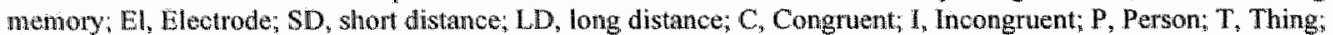
GG, Greenhouse Gelsser conected $\mathrm{p}$-value for $\mathrm{F}$ tests with mone than one degree of freedom. Table displays only ANOVA tests which are most relevant. All main effects that are not reported are not significant (NS). The same holds for the following table.

In contrast, the SD-thing condition does not show a difference in the $200-400$ ms time window. Rather, from $400 \mathrm{~ms}$ after pronoun onset and extending well into the ERP of the next word, the ERP to incongruent stimuli is more positive than that to congruent SD-thing 
pronouns. The LD conditions show a different ERP pattern. In both antecedent conditions in LD, the ERP waveforms are similar in the $200-400 \mathrm{~ms}$ time window. For person pronouns in LD, there is difference in the $400-700 \mathrm{~ms}$ range with incongruent pronouns being more positive than congruent pronouns whereas this effect is absent for $L D$ thing pronouns.

Figure 5.3 and Figure 5.4 compare ERPs to pronouns for short and long distance sentences. For pronouns referring back to persons (Figure 5.3, upper panel) there is a difference in congruent pronouns with the LD condition being more negative than the SD condition between 200 and $700 \mathrm{~ms}$. In contrast, incongruent person-pronouns (lower panel) show a difference in the $\mathrm{P} 600$ range $(400-700 \mathrm{~ms})$ with $\mathrm{LD}$ condition being more positive than SD. For thing-antecedents (Figure 5.4) a negativity for LD pronouns compared to SD pronouns is found between 200 and $450 \mathrm{~ms}$ for congruent (upper panel) and between 200 and $700 \mathrm{~ms}$ for incongruent pronouns (lower panel). The latter effect was seen mostly at parietal sites.

Statistical analysis is reported separately for the N400 and P600 latency range. Onnibus and separate ANOVAs are reported in Table 5.2 for the N400 range and in Table 5.3 for the P600 range.

\section{Early negativity: $200-400$ ms}

Our pronoun manipulation showed a main effect for Working memory in an omnibus ANOVA (see Table 5.2) plus interactions of Working memory with Electrode, as well as with Congruency $x$ Electrode. As can be seen in Figure 5.5 the interaction (Working memory $x$ Congruency $x$ Electrode) is mainly due to the fact that congruent and incongruent conditions differ in SD but not in LD memory sentences. Further analysis showed that this holds for the person sentences, and not for the thing sentences, as was confirmed by separate analyses (Table 5.2, Congruency) revealing a significant main effect for congruency for SD person sentences with incongruent pronouns being more negative than congruent pronouns. In contrast, separate analyses for SD thing sentences did not reveal a main effect for congruency, nor for person and thing LD conditions.

Separate ANOVAs comparing SD and LD sentences are reported in Table 5.2 as well (verbal working memory). Here, statistical analysis revealed a main effect for congruent personpronouns with LD conditions being more negative than SD conditions. This effect was absent in all other conditions. 


\section{Congruent Person}
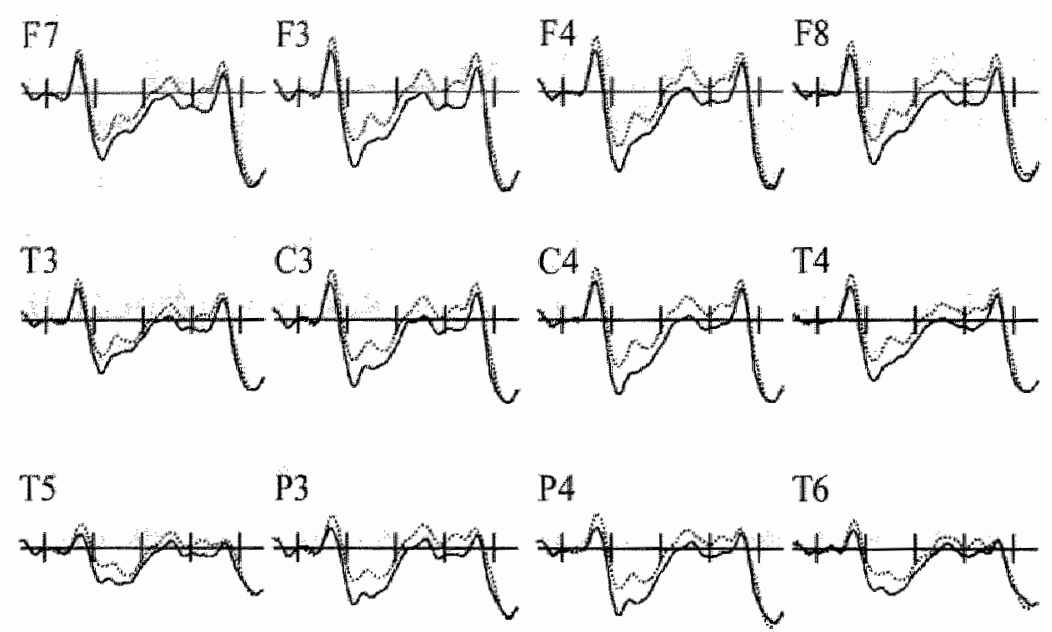

\section{Incongruent Person}

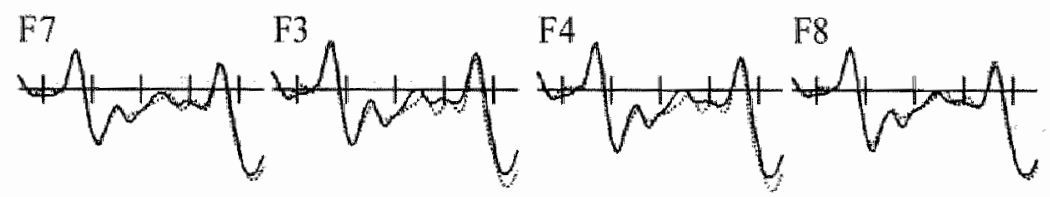

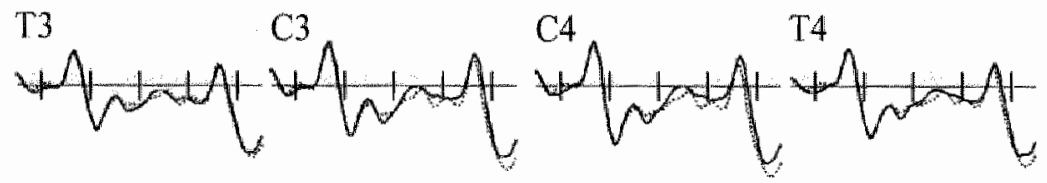
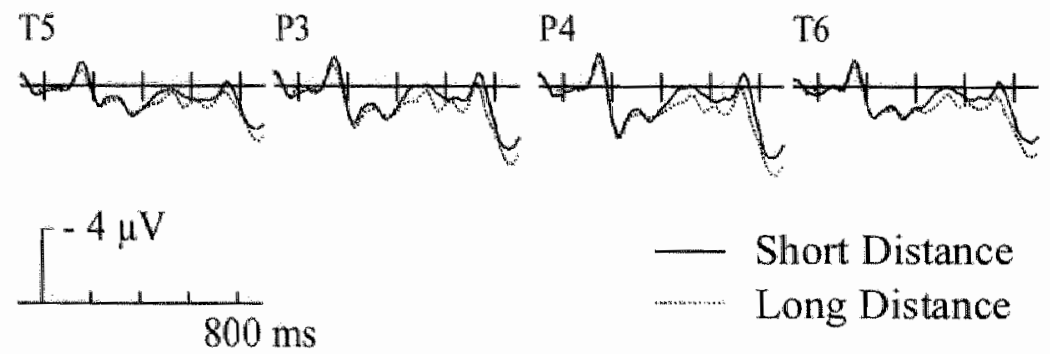

Figure 5.3 Grand average ERPs time locked to the onset of Person-pronouns for congruent SD and LD sientences (apper panel) and incongruent SD and LD sentences (lower panel) at a selected set of electrodes. 


\section{Congruent Thing}
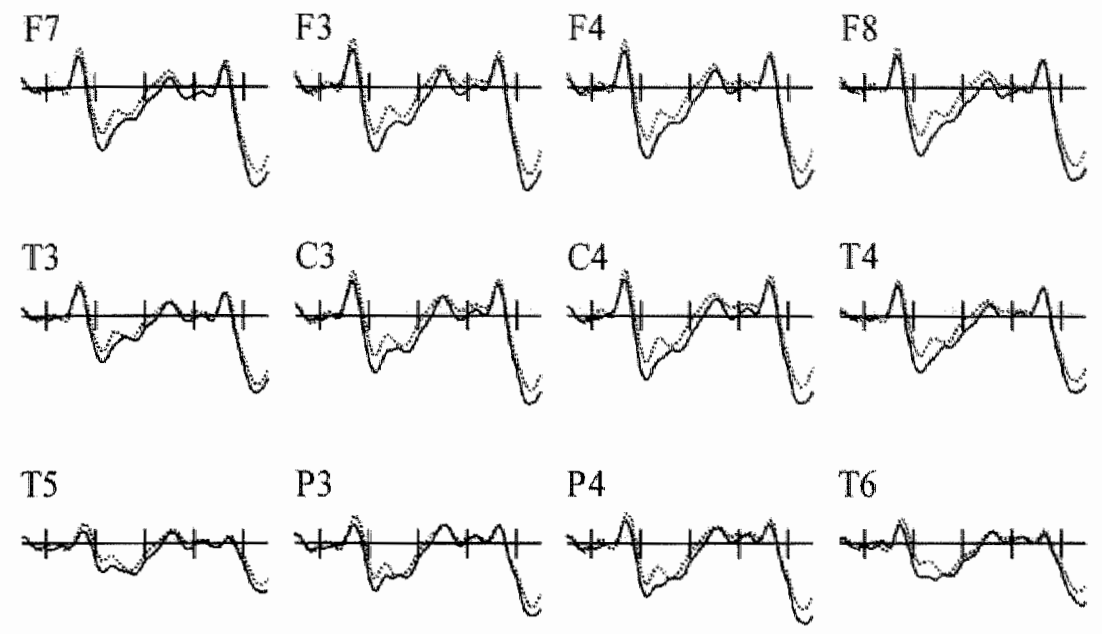

\section{Incongruent Thing}
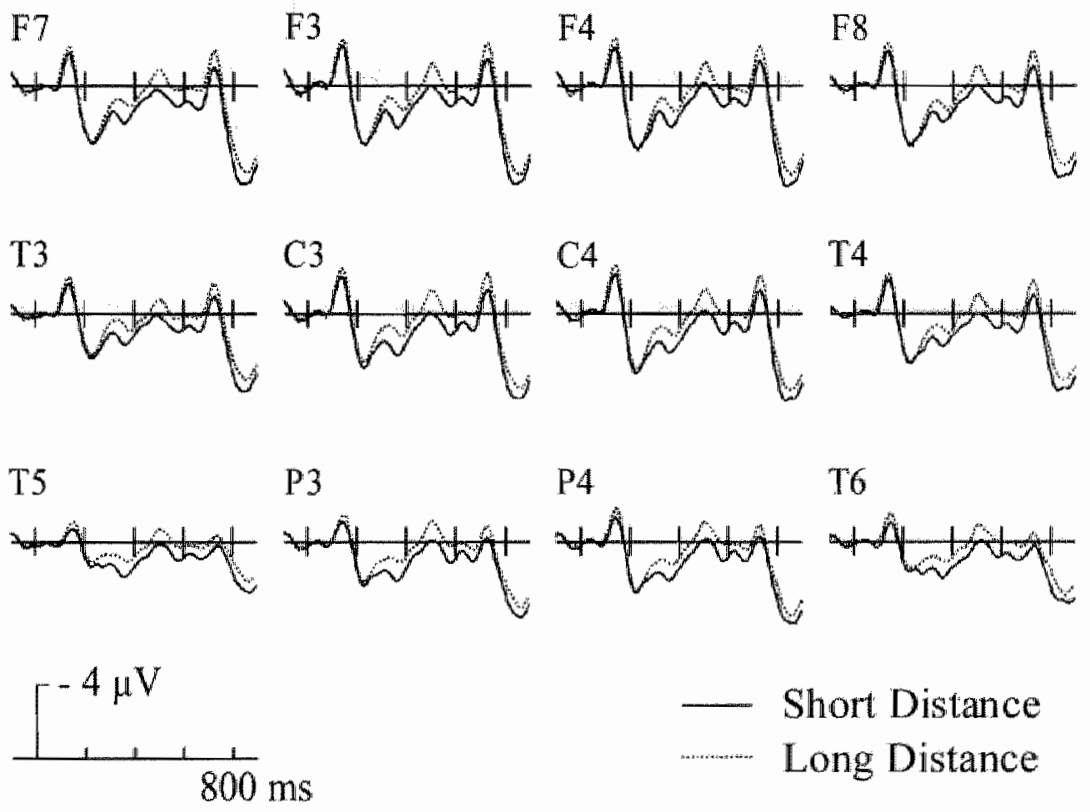

Figure 5.4 Grand average ERPs time locked to the onset of Thing-pronouns for congruent SD and LD sentences (upper panel) and incongruent SD and LD sentences (lower panel) at a selected set of electrodes. 

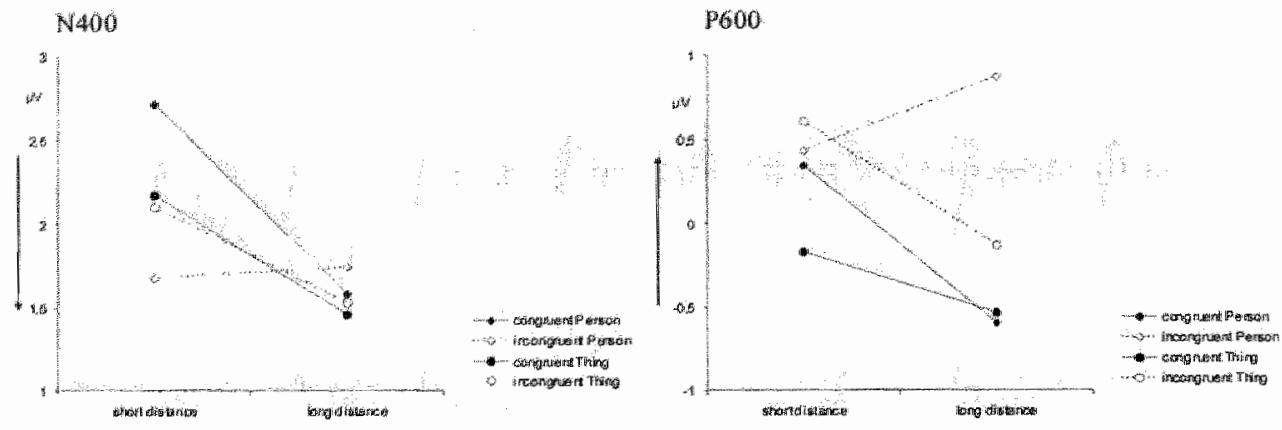

Figure 5.5 Mean amplitudes of the ERP waves for congruent and incongruent Person antecedents and for congruent and incongruent Thing antecedents during SD and LD pronoun resolution are demonstrated for the $\mathrm{N} 400$ (timewwindow 200-400 ms, left panel) and for the $\mathrm{P} 600$ (time-window $400-700$, right panel).

\section{P600: $400-700 \mathrm{~ms}$}

The omnibus ANOVA with 29 electrodes (Table 5.3) revealed a significant main effect for Congruency (congruent vs. incongruent), indicating a modulation of the P600 for our pronoun manipulation. Furthermore, we found a triple interaction Memory $x$ Antecedent $x$ Congruency, as well as an interaction between Memory and electrodes. As can be seen in Figure 5.5, this interaction is due to the fact that in the SD condition things but not persons are sensitive to a congruency manipulation. In contrast, in LD the opposite scenario is the case: persons but not things are sensitive to congmency. These effects were traced back by separate analyses (see Table 5.3, lower part) which revealed the following results: a main effect for congrtuency to thing pronouns in the SD conditions (incongruent more positive than congruent pronouns) and a main effect for congruency to person pronouns in the $L D$ conditions (incongruent being more positive than congruent pronouns).

With respect to working memory effects statistical analysis revealed a main effect for congruent person-pronouns with LD conditions being more negative than SD conditions (see Figure 5.3). This seems to be a continuation of the negative effect reported above. As in the early time window, separate analyses for the other three conditions did not reveal any significant differences between LD and SD type sentences. 


\begin{tabular}{|c|c|c|c|}
\hline Source & $\overline{\mathrm{df}}$ & F & $\mathrm{P}(\mathrm{GO})$ \\
\hline \multicolumn{4}{|l|}{ Omnibus ANOVA (29 electrodes) } \\
\hline Congruency (congruent vs. incongruent) & 1,15 & 8.07 & .01 \\
\hline VWM $x$ Antecedent $x$ Congruency & 1,15 & 6.02 & .03 \\
\hline VWM $\times \mathrm{El}$ & 28,420 & 4.34 & .004 \\
\hline \multicolumn{4}{|c|}{ Separate ANOVAs for Congruency (29 electrodes): } \\
\hline SD P: Congruency (congruent ws. incongruent) & 1,15 & 0.09 & \\
\hline Congruency $x \mathrm{El}$ & 28,420 & 1.06 & \\
\hline SD T: Congruency (congruent vs. incongruent) & 1,15 & 4.79 & .04 \\
\hline Congruency $\times \mathrm{EI}$ & 28,420 & 1.77 & \\
\hline LD P: Congruency (congruent vs. incongruent) & 1,15 & 11.57 & .004 \\
\hline Congruency $\times \mathrm{E}$ & 28,420 & 3.38 & .047 \\
\hline LD T: Congruency (congruent vs. incongruent) & 1,15 & 0.52 & \\
\hline Congruency $\mathrm{x} \mathrm{El}$ & 28,420 & 0.65 & \\
\hline \multicolumn{4}{|c|}{ Separate ANOVAs for Working Memory (29 electrodes): } \\
\hline CP: VWM (short vs. long) & 1,15 & 6.67 & .02 \\
\hline$V W M \times E l$ & 28,420 & 3.71 & .005 \\
\hline IP: VWM (short ws. long) & 1,15 & 1.36 & \\
\hline VWM $\times \mathrm{EI}$ & 28,420 & 3.07 & .009 \\
\hline CT: VWM (short vs. long) & 1,15 & 0.61 & \\
\hline VWM El & 28,420 & 1.46 & \\
\hline IT: VWM(short vs. long) & 1,15 & 3.03 & \\
\hline VWM $\times E l$ & 28,420 & 1.01 & \\
\hline
\end{tabular}

Table 5.3 Mean. ERP amplitude ANOVAs in the $400-700 \mathrm{~ms}$ Latency Range (P600). Mote: VWM,

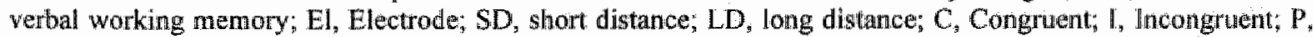
Person; $T$, Thing; GG, Greenhouse Geisser corrected $p$-value for $F$ tests with more than one degree of freedom. 


\begin{tabular}{|c|c|c|c|}
\hline Source & $\mathrm{df}$ & F & $\mathrm{P}(\mathrm{GO})$ \\
\hline \multicolumn{4}{|l|}{ Omuibus ANOVA (29 ellectrodes) } \\
\hline Congrisency (congruent vs incongruent) & 1,15 & 4.89 & .04 \\
\hline Congruency $x$ Electrode & 28,420 & 3.58 & .006 \\
\hline VWM (short ws, long) $x$ Electrode & 28,420 & 4.49 & .008 \\
\hline \multicolumn{4}{|c|}{ Separate ANOVAs for Congruency (29 electrodes): } \\
\hline SD P: Congruency (congruent vs. incongruent) & 1,15 & 0.28 & \\
\hline Congruency $x \mathrm{E}$ & 28,420 & 1.80 & \\
\hline SD $T$ : Congruency (congnient $w s$, incongruent) & 1,15 & 3.61 & .08 \\
\hline Congruency $\times \mathrm{E}$ & 28,420 & 2.71 & .04 \\
\hline LD P: Congruency (congruent vs. incongruent) & 1,15 & 0.17 & \\
\hline Congruency $\mathrm{x} \mathrm{El}$ & 28,420 & 0.88 & \\
\hline LD T: Congruency (congruent vs. incongruent) & 1,15 & 2.68 & \\
\hline Congruency $\times \mathrm{EI}$ & 28,420 & 1.43 & \\
\hline
\end{tabular}

Frontal ( $\mathrm{F} 3, \mathrm{~F} z, \mathrm{~F} 4)$ ANOVAs for Congruency (congruent $v s$. incongruent)

$\begin{array}{llll}\operatorname{SDP} & 1,15 & 0.44 & \\ \operatorname{SDT} & 1,15 & 4.65 & .047 \\ \operatorname{LDP} & 1,15 & 0.60 & \\ \operatorname{LDT} & 1,15 & 4.35 & .05\end{array}$

Central (C3, $\mathrm{Cz}, \mathrm{C4})$ ANOVAs for Congruency (congruent vs. incongruent)

$\begin{array}{lll}\mathrm{SDP} & 1,15 & 0.71 \\ \mathrm{SDT} & 1,15 & 4.77 \\ \mathrm{LDP} & 1,15 & 0.43 \\ \mathrm{LDT} & 1,15 & 2.71\end{array}$

Frontal (P3, $\left.\mathbb{2} \%, P_{4}\right)$ ANOVAs for Congruency (congruent vs. incongruent)

$\begin{array}{lll}\operatorname{SDP} & 1,15 & 0.45 \\ \operatorname{SDT} & 1,15 & 2.00 \\ \mathrm{LDP} & 1,15 & 0.00 \\ \mathrm{LDT} & 1,15 & \mathbb{1} .84\end{array}$

Tuble 5.4 Mean ERP amplitude ANOVAs in the $1000-1300$ ms Latency Range (late N400). Note: VWM, verbal working menory; El, Electrode; SD, short distance, LD, long distance; $\mathrm{P}$, Person; $T$, Thing; GG, Greenhouse Geisser corrected p-value for F tests with more than one degree of freedom. 
$\mathrm{A}$

Short Distance

Person

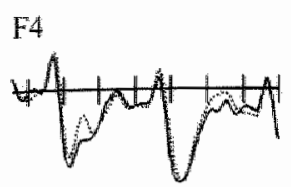

प4

P4

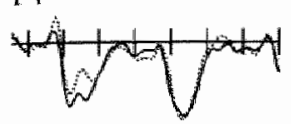

$\mathbf{P} 4$

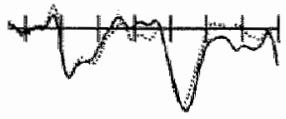

Thing

F4
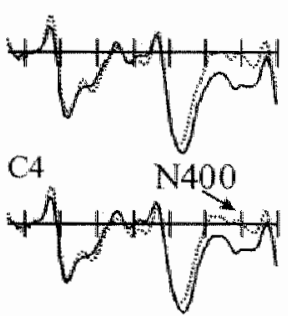

B

Congruent

Incongruent
Long Distance

Person

Thing
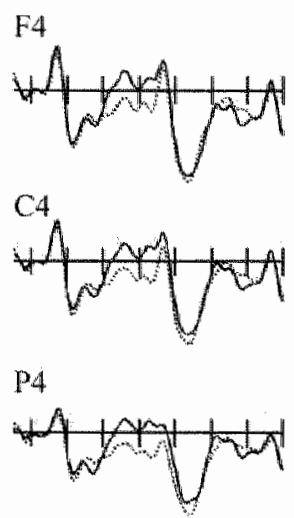

$\mathrm{F} 4$
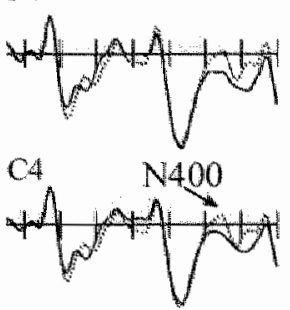

P4
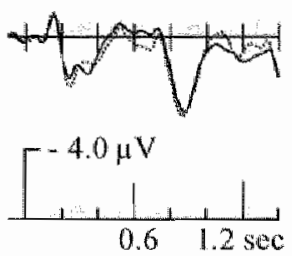

Figure 5.6 Grand average ERPs time locked to the onset of Person-pronouns (left) and Thing Pronouns (right) for SD conditions (Panel A) and for LD conditions (Panel B) at a selected ser of electrodes.

\section{Negativity at follow-up word: $1000-1300 \mathrm{~ms}$}

Figure 5.6 shows the grand average ERPs, time-locked to the onset of the pronoun up to the end of the following word for SD person and thing conditions (panel A) and LD person and thing conditions (panel B). In addition to the ERP-patterns described before Figure 5.6 shows a more negative waveform for the word following the pronoun ( reif") in incongruent sentences about things compared to congruent sentences in SD-conditions. This negativity begins approximately at $1000 \mathrm{~ms}$ and lasts up to $1300 \mathrm{~ms}$ (corresponding to $400-700 \mathrm{~ms}$ after word onset) with an anterior maximum. LD thing-conditions reveal a similar pattern with incongruent sentences being more negative than congruent sentences, but with a reduced effect size. However, these patterns are not visible for person sentences in SD and LD conditions.

Table 5.4 revealed that our pronoun manipulation in an omnibus ANOVA crossing factors Working memory, Antecedent, Congruency and Electrodes showed a significant main effect for Congruency and an interaction for Congruency $x$ Electrode and VWM $x$ Electrode in the 1000-1300 ms time window. Separate analysis splitting Person and Thing antecedents in SD and $\mathrm{LD}$ conditions revealed a main effect for congruency for $\mathrm{SD}$ thing-condition, indicating an $\mathrm{N} 400$ effect. Neither $\mathrm{SD}$ and $\mathrm{LD}$ person nor $\mathrm{LD}$ thing conditions reached significance. 
Further topographical analysis showed that SD thing conditions reached significance at frontal and central sites. Moreover, $L D$ thing conditions showed a trend towards a frontal negativity within the specified time-window.

\subsection{Discussion}

The aim of this experiment was to investigate the interaction of verbal working memory with semantic and/or syntactic information within the process of establishing co-reference between a pronoun and its antecedent. Previously, ERP effects suggestive of the involvement of syntactic information (P600; Osterhout \& Mobley, 1995; Schmitt et al., 2002), semantic information (N400; King \& Kutas, 1995) or both (N400/P600 complex; Schmitt et al., 2002) have been reported. Interactions of verbal working memory and semantic and/or syntactic information processing may be observed via differences of the N400 (semantic) and P600 (syntax) components between conditions with less demand on VWM (short distance) and more demand on VWM (long distance).

We applied a design that allowed us to study pure syntactic gender disagreement in comparison to combined semantic/syntactic violations (biological gender disagreement) using person or thing as antecedents. Additionally the present design allowed assessing the influence of different working memory demands on pronoun resolution. Such demands have been shown to give rise to a LAN component (Kluender \& Kutas, 1993; Streb et al. 1999; van Berkum et al., 2003). While we did observe an enhanced negativity for $L D$ relative to SD conditions with an onset latency of about $200 \mathrm{~ms}$ in three out of four conditions (congruent/incongruent thing, congruent person), these effects did not resemble a LAN component as they were rather widespread (Figures 5.3 and 5.4). At present, we are unable to determine a direct relationship of the negativities found between LD and SD conditions and the LAN reported in previous studies. We can only interpret the outcome based on our design assumptions. The only difference in the comparison is the distance between antecedent and pronoun. We therefore suggest that the observed negativity reflects the more difficult establishment of co-reference in a LD compared to a SD condition. The findings show that the system processes this difference within $200 \mathrm{~ms}$ after pronoun onset. We can only speculate on the meaning of this, but we think that this difference reflects a relatively easy conceptual reactivation of the antecedent in the SD compared to more difficult one in the LD condition. 
The comparison of the ERPs to incongruent and congruent pronowus for the different conditions (Figures 5.1 and 5.2) allowed us to assess the influence of working menory demands on the N400 and P600 components ellicited by the gender violations. Efrects of working memory were found for both components, thus showing that VWM interacts with semantic and syntactic processing during the establishment of pronounantecedent coreference.

Semantic integration and verbal working memory: As indicated in Figure 5.5 (left panel), we found an N400 effect for the SD Person condition only. ${ }^{2}$ This finding can be interpreted such that VWM interacts with semantic gender information processing in sentences with person antecedents. In contrast, pronouns with thing sentence types did not show any N400 effect. This came as no surprise, as only syntactic gender processing was manipulated and therefore no semantic integration problems were involved for the violation conditions.

Why is there no N400 in the person LD condition? Figure 5.5 suggests that in LD condition the establishment of co-reference involving biological/semantic gender was so diffeult that a ceiling effect was reached for the N400 effect: Semantic integration is not an all-or-none process (e.g. van Petten \& Kutas, 1991). For example, a large N400 can be observed for sentence initial content words, in spite of the fact that these are perfectly sensible in that position. We propose that the LD manipulation raised semantic integration difficulty in the congruent person condition such that congruent and incongruent person conditions were indistinguishable for the $\mathrm{N} 400$ process. This idea is also supported by the observed negativity in the comparison of congruent $\mathrm{LD} / \mathrm{SD}$ conditions (see previous page). In order to be able to link an incoming pronoun to a potential antecedent, possible antecedents are supposed to be re-activated (Nicol \& Swinney, 1989; MacDonald \& MacWhinney, 1990). We argue that this reactivation is probably more difficult for antecedents which are further away from the pronoun compared to close antecedents.

Syntactic integration and verbal working menory: As expected, we found a more positive ERP for incongruent compared to congruent pronouns for thing sentences in SD within the

\footnotetext{
Why did we observe different outcomes for SD person sentences compared to the person sentences reported in Chapter 2 ? There, we reported a large P600 whereas here the very same naterial elicited an N400. One possible explanation would be that the comprehension systend did react sensitive to the change in experimental set up. Adding more complex sentences (LD) to the experiment changed the assessment of difficulty for these sentence. Treating them as easier to solve compared to LD leads into a general difference in assessment for the sanne material ewoking different processing as compared to singled presentation of these sentences.
} 
$400-700 \mathrm{~ms}$ time range. Furthermore, we found a P600 for person sentences in LD with incongruent pronouris being more positive than congruent. This is shown in Figure 5.5 (right panel). Additionally, there seems to be a P600 for thing sentences within the LD conditions, but this effect failed to reach significance. For the interpretation of this pattern of results we need to take into account current knowledge about the $\mathrm{P} 600$ effect. To reiterate the findings in the literature, an increase in P600 amplitude has been found in response to several kinds of syntactic violations such as subject-verb agreement violations (Hagoon et al. 1993; Osterhout \& Mobley, 1995), verb inflection errors (Friederici et al. 1993; Gunter, Stowe \& Mulder, 1997), case inflection errors (Münte et al., 1998a), and phrase structure violations (Friederici et al., 1999; Hahne \& Friederici, 1999; Neville et al., 1991). In addition, P600 effects have been found for the less preferred structure in so-called 'garden path' sentences (e.g. The woman persuaded to answer the door...; Osterhout \& Holcomb, 1992). Finally, Kaan, Harris, Gibson and Holcomb (2000) found P600 effects to grammatically correct, non-garden path sentences, which were hard to integrate. These results have led researchers to assume that the P600 amplitude is related to "syntactic processing difficulty" and that this phenomenon might be related to syntactic reanalysis (Osterhout et al., 1994; Rösler, Pechman, Streb, Röder \& Hennighausen, 1998), syntactic integration difficulty (Kaan et al., 2000), or the inability to assign the preferred structure to the words encountered (i.e. Friederici et al., 1999). Such syntax-specific views are challenged by findings of a P600-like effect for rare semantic violations within long stories (Munte et al., 1998a), semantic reversal anomalies (The cat that fled from the motse ran across the room. Kolk, Chwilla \& van Herten, 2003), or semantic selectional restriction violations (For breakfast the eggs would only eat ...; Kuperberg, Holcomb, Sitnikova, Greve, Dale \& Caplan, 2003).

Hagoort (2003) has recently provided an account of the processes underlying the P600 component drawing heavily from the 'Unification model', a computational model formulated by Vosse and Kempen (2000). The key concept of this model is that each word in the lexicon is associated with a structural frame that specifies the possible structural environment of this particular word. In the process of sentence comprehension, the syntactic frames are unified into a structural representation of the whole sentence by the formation of 'unification links'. In Hagoort's view, the amplitude of the P 600 varies as a function of the difficulty of forming unification links. Factors determining the difficulty of this formation process include the presence of alternative unification options, syntactic complexity, as well as semantic influences. For example, expectancies about what is (im)possible in the real world, could hamper the formation of unification links, and thus produce a P600 effect, which would be 
consistent with the various results indicating a P600 for semantic volations (Kolk et al., 2003; Kuperberg et all, 2003; Münte et al., 1998a).

Thus, sentence processing may underlie a hierarchical structure, and this should hold for building up co-reference between a pronoun and an antecedent as well. In case of a person antecedent within easy sentences the comprehension system relies on semantic gender information as reported above (N400 for incongruent compared to congruent SD person pronouns). In contrast, if only syntactic gender information is available as for pronouns referring back to thing antecedents in SD sentences, a P600 was observed indicating syntactic re-analysis. In case of more complex sentences, i.e. increased distance between pronoun and antecedent (LD conditions), the parser brings into play a different processing architecture. For LD person pronouns a direct index of semantic integration problems is absent (no N400) indicating a weaker reflex of independent semantic processing. Instead, the parser rather seems to rely on syntactic gender information indexed by a P600 component. Additionally, this $\mathrm{P} 600$ was larger for LD person pronouns compared to LD thing pronouns indicating more processing demands for building an unification link (Hagoort, 2003) based on increased distance and the violation of two types of gender information (biological and syntactic). In contrast, we did not observe a P600 for incongruent LD thing pronouns. Here, we speculate that the thing is not considered as the belonging antecedent to the pronoun due to the increased distance, at least not at pronoun position. Therefore, it might be fruitful to consider the observed effects on the word following the pronoun to take account of possible ongoing searching processes (Hammer et al., 2005). We found a late N400-like effect on the word following the pronoun within incongruent sentences about things compared to congruent. This effect was seen in SD and LD conditions (Figure 5.6). This effect was most salient at anterior sites. In contrast, no such pattern was found for SD or LD person sentences. This N400 has been shown to be sensitive to semantic integration (Kutas \& Hillyard, 1980, 1983; Kutas \& van Petten, 1994), as well as to discourse integration (St. George et al., 1999; Van Berkum et al., 1999). The observed effect therefore indicates an involvement of semantic (world knowledge of stereotypes) and/or discourse processing of words during the integration of the pronoun into the sentence. The anterior distribution of our $\mathrm{N} 400$-like effect could be related to the anterior negative effect for discourse-referential ambiguity reported by Van Berkum and colleagues (2003). This effect is consistent with the earlier finding of our group for the SD thing sentences (Hammer et al, 2005), which even holds for LD thing-conditions. Thus, the effect within thing sentences could be interpreted as an ongoing integration process in order to get the meaning of the pronoun right. This revision might be characterized by a searching 
process for an alternative antecedent within the discourse. Therefore, the parser waits for new information in order to solve the incongruity, i.e. expecting a relation to an alternative antecedent in the discourse. The N400 occurs, if this process fails. In contrast, in the personcase, no N400 was found. Therefore, we can conclude that a revision does not take place at the word following the pronoun indicating that the integration process for these pronouns seems to be finished right after pronoun processing due to a reliable declining of bonding/resolution of pronoun and person-antecedent. This seems to be independent of the distance between pronoun and antecedent.

\section{Conclusions}

Thus, these data show that verbal working memory (VWM) and semantic and syntactic violation do interact. In a relatively less demanding VWM condition, the parser seems to have the preference to use semantic information to establish co-reference if this information is available, as in our person sentence types (biollogical gender information). It can distinguish between congruent and incongruent gender types (shown as a N400 and a lack of P600 in our $\mathrm{SD}$ person condition). If semantic gender information is not available (as for things in German), the syntactic parsing takes over because of its unambiguous syntactic information for German things (shown as the P600 in our SD thing condition). If there are more demands on VWM as in the LD conditions, the parser seems to rely on syntactic information rather than on semantic one (shown as a P600 and lack of N400 in the LD person condition). However, a high demand on VWM also interacts with pure syntactic parsing in such a way that a syntactic gender violation cannot be detected anymore (lack of P600 in our LD thing condition) at pronoun position. Furthermore, we found an anterior N400-like component on the word following the pronoun solely in Thing conditions, indicating an ongoing integration process for these conditions, which is independent of distance. In contrast, for Personsentences the integration process is finished right after pronoun position, indexed by similar ERP waves on the word following the pronoun for incongruent and congruent sentences:

In general, these data suggest that sentence parsing is not only semantic or syntactic but rather a highly tlexible process. Our different N400/P600 patterns suggest that a parser switches preference between semantic and syntactic information access during pronoun processing depending on demands on VWM resources and information availability. 


\title{
Interaction of semantic and syntactic gender information and working memory during pronoun resolution: an fMRI study
}

\begin{abstract}
Personal pronouns are used to refer back to an earlier mentioned person or thing. In German, this re-reference an be based on syntactic and/or semantic gender information. The aim of this functional Magnetic Resonance imaging study was to localize syntactic and semantic information and possible interaction with verbal working memory (VWM) during the process of building up co-reference between a pronoun and its antecedent using the same material as in the study described in chapter five. We experimentally disentangled syntactic and biological/conceptual gender information by using German sentences either about a person (biological and syntactic gender assignment) or about a thing (syntactic gender assignment) followed by a congruent or incongruent pronoun in terms of gender. Additionally we manipulated VWM by increasing the distance across noun phrases between pronoun and antecedent resulting in a short distance (SD) and a long distance (LD) VWM condition. As a main result we found that left inferior frontal regions are the core region for building wa $\mathrm{co}^{-}$ reference between pronoun and antecedent with a slight local differentiation dependent on antecedent type. In addition, we suggest that left superior frontal regions may be ascribed as a general monitoring processor for clear gender violations within comparably easy sentences (SD) as we found deactivation for incongruent compared to congruent pronouns within these regions but not for LD sentences. Furthermore, possible search processes for alternative antecedents within LD thing sentences were reflected by activation of temporal and lemporo-parietal regions. Furthermore, we demonstrated sensitivity to the distance manipulation depending on availability of gender information within congruent sentences, which was reflected in a temporal activation for LD compared to SD Person sentences, whereas for the thing case no temporal activation was found. For incongruent sentences we found a difference for antecedent types within temporal regions, with an anterior temporal activation for Person antecedents and a more posterior temporal activation for Thing antecedents. We suggested that these areas get active due to a search for an appropriate antecedent depending on information availability in case of violations, but also, in case of longer temporal distance (LD) between antecedents and pronouns.
\end{abstract}




\subsection{Introduction}

The present study focuses on patterns of brain activations associated with syntactic and semantic gender information processing and possible interactions with verbal working memory (VWM) during pronoun resolution. Reading a sentence like 'The woman is happy because she fell in love." it is easy to tell that "she' refers back to the woman. In order to be able to integrate the pronoun 'she'. into the sentence, it has to be re-referenced to the antecedent (i.e. the woman). Thus, the antecedent is supposed to be re-activated (Nicol \& Swinney, 1989; MacDonald \& MacWhinney, 1990) possibly by using VWM resources. Building up co-reference between pronoun and antecedent may be based on syntactic and/or biological/semantic gender information of the two. The woman in our example is characterized by female biological gender information. In German, this biological gender information is accompanied by feminine syntactic gender information (die feminine

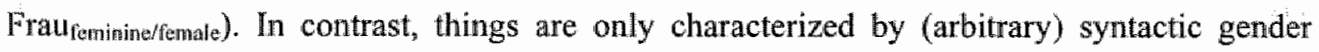
information without any semantic reflex ( $\mathrm{die}_{\text {feminine }}$ Tasche $_{\text {ferninine }}$ ). These characteristics of the German language enable us to systematically manipulate the process of syntactic and semantic gender information integration during pronoun processing. Now consider the sentence 'The woman was happy and kept on smiling because she fell in love.' Here, the antecedent is further away from the pronoun. Therefore, the process of building up coreference between the pronoun and belonging antecedent taxes more VWM resources because it either has to be kept available in VWM for a Jonger time or it has to be re-activated at a later time point compared to the shorter sentence. In addition to the processing of gender information, we focus on possible interaction of these with verbal working memory by varying the distance between pronoun and antecedent.

In chapter four, the reader finds an overview on recent investigations concerning imaging studies examining syntactic and semantic information processing (pp. 51-54), which will be summarized in the following. Syntactic processing was found to be located in an area of the left dorsolateral prefrontal cortex (Indefrey et al, 2001). Semantic processing revealed a more complex network including the bilateral inferior frontal and inferio-medial temporal cortex, left lateral frontal cortex, left posterior fusiform gyrus, bilateral motor cortex, and supplementary motor area (Kiehl et al.,2002). A more recent study revealed that the inferior prefrontal cortex is involved in integration of meaning (semantic) and world knowledge (Hagoort et al., 2004). Newman and colleagues (2001) investigated syntactic and semantic 
processing within one study and reported that syntactic and semantic processing result in nonidentical patterns of activation, including greater frontal engagement during syntactic processing and larger increases in temporal and temporo-parietal regions during semantic analyses. In a completely crossed design, Röder and colleagues (2002) varied syntactic processing demands (easy vs. difficult to process word order sequences) and the meaningfulness of sentences (real- vs. pseudo-word sentences). Semantic in contrast to pseudo-word sentences elicited a stronger activation in inferior frontal gyrus, and superior and middle temporal gyrus. Syntactic difficulty had its strongest effect within the left inferior frontal region and this effect was more pronounced for semantic than non-semantic speech. The authors interpreted their findings that semantic and syntactic language functions are mediated by partly specialized brain systems but that there is a substantial functional overlap of the involved brain structures, especially in left inferior frontal regions. Friederici and colleagues (2003) investigated brain areas supporting the processing of sentence-level semantic and syntactic information, finding that both processes are supported by a temporofrontal network with distinct areas specialized for semantic (mid-portion of the superior temporal region bilaterally and the insular cortex bilaterally) and syntactic processes (anterior portion of the left superior temporal gyrus, the left posterior frontal operculum (adjacent to Broca's area) and the putamen in the left basal ganglia).

Imaging studies on Working Memory (WM): Cooke and colleagues (2001) investigated the where of syntactic working memory with AMRI by presenting sentences differing in grammatical structure (subject- ws. object-relative) and their short-term memory demands (short vs. long antecedent-gap linkages). Subjects were asked to consider whether a male or a female person performed an action. There were two main results. First, the right posterior superior temporal cortex showed activation during processing of long distance antecedent-gap without any grammatical influence in comparison to a pseudofont baseline. "The authors took this finding as this area supports passive short-term memory during sentence comprehension. Second, the left inferior frontal cortex was associated with sentences that featured both an object-relative clause and a long antecedent-gap in comparison to the baseline. This suggests that this region supports the cognitive resources required to maintain long-distance syntactic dependencies during comprehension of grammatically complex sentences. Fiebach, Schlesewsky, and Friederici (2001) found comparable results for German short and long whquestions differing in their grammatical function (subject vs. object wh-question). 
Martin and Chao (2001) presented a review on semantic memory. They concluded based on i.e. Damasio"s work (1996) that the temporal lobe object representation system is organized hierarchically, with increasing convergence and integration of information (from generic to unique) occurring along its posterior to anterior axis. Furthermore, they stated that evidence has accumulated suggesting that an anterior and inferior prefrontal region (roughly equivalent to $\mathrm{BA} 47$ and the inferior aspect of $\mathrm{BA} 45$ ) may be involved selectively in semantic processing. Specifically, as suggested by Gabrieli, Poldrack, and Desmond (1998) and Wagner (1992), this region may serve as a "semantic working memory system" responsible for retrieving, maintaining and manipulating semantic representation stored elsewhere.

In a more recent study, Thierry, Ibarrola, Démonet, and Cardebat (2003) investigated the involvement of the inferior prefrontal cortex in VWM (which is the subsystem for working memory devoted to verbal information (Baddely, 1998)). Subjects listened to pairs of French words. Half of the presented words corresponded to artifacts, the other half to natural things. Each semantic group featured an equal number of feminine and masculine nouns. Subjects' task was to monitor the pairs for a category. Within a pair, both words pertained to a specific category (feminine, masculine, natural, artificial). First, the authors could vary VWM demands by manipulating the number of verbal items (one or two), which needed to be categorized. Either it was possible to make a decision without processing the second word (rellease condition) or the second word had to be held in VWM in order to respond (hold condition). Second, two different language tasks were used, a gender decision (syntax categorization into feminine and masculine) and a semantic decision (categorization into artificial and natural). Therefore, the authors claimed to test whether VWM would interact with the linguistic process in focus. The authors reported the following main results: (1) Reaction times were faster in release than hold conditions with gender decision being faster than semantic decisions. (2) Activation patterns were remarkably similar across tasks and conditions, revealing a wide bilateral network, i.e. including the superior temporal gyrus, inferior prefrontal gyrus, thalami, and lingual gyri. The authors concluded that the performed cognitive operations (in particular access to meaning of the words) were too similar to allow different networks to be involved. (3) A significant hemodynamic peak delay between hold and release condition was identified in the inferior prefrontal regions but not in superior temporal regions, and this effect did not interact with the language task. This finding indicated that superior frontal regions showed no sensitivity to a condition change, whereas the major factor influencing the time course of the inferior prefrontal cortex hemodynamic response was verbal working memory rather than the linguistic task in question. 
To summarize the above studies, we look for common findings between them. Syntactic processing was dominantly located in prefrontal regions (Indefrey et al., 2001; Newman et al., 2001; Röder et al., 2002 and Friederici et al., 2003). In addition, Friederici and colleagues (2003) reported an activation of the anterior portion of the left superior temporal gyrus and the putamen for symtactic processing. However, semantic processing was dominantly located within inferior frontal regions (Kiehl et al, 2002; Newman et al, 2001; Roder et al., 2002; Friederici et al., 2003 [insular cortex]; and Hagoort et al., 2004) and superior and/or middle temporal regions (Kiehl et al, 2002; Newman et al.; 2001; Röder et al., 2002; Friederici et all., 2003). With respect to syntactic WM (i.e. maintaing long distance syntactic dependencies) the involvement of the left inferior frontal cortex was reported (Cooke et al.; 2001, Fiebach et al., 2001), as well as for a 'semantic WM system' (Gabrieli et al., 1998; for a review see Martin and Chao, 2001). However, temporal lobe was titled as an object representation system (Damasio, 1996). This was supported by the work of Thierry and colleagues (2003), who reported similar activation patterns for semantic and syntactic processing, including the superior temporal gyrus, inferior prefrontal gyrus as well. In addition, they reported that a higher demand on WM delays the time course of the inferior prefrontal cortex hemodynamic response, whereas the superior temporal gyrus was insensitive to this effect.

The aim of the present fMRI study was to localize corresponding neuronal areas to semantic integration and syntactic integration and whether they would interact with verbal working memory during establishing co-reference between a pronown and the corresponding antecedent in sentences. We used a passive reading task in a standard violation paradigm to ayoid effects of explicit cognitive decision tasks. Our paradigm allows us to investigate syntactic and semantic information processing based on the gender type of the antecedent. In one condition a person was introduced as an antecedent and later referred to by a pronoun, which either agreed in biological/syntactic gender or not biological/syntactic gender violation). In a second condition, a thing was introduced as an antecedent and the corresponding pronoun either agreed in syntactic gender with the antecedent or not (syntactic gender violation). In addition, we manipulated verbal working memory (WM) in pronoun processing for both linguistic conditions by increasing the distance between antecedent and pronoun. Thus, the distance was either short (short distance $=\mathrm{SD}$ condition) or long (long distance $=$ LD condition, see Table 6.1 for example material $)$. 


\section{Conditum Sentence}
SD P: Person as ontecedert

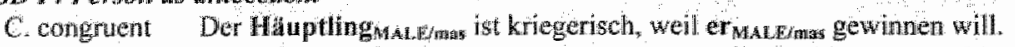

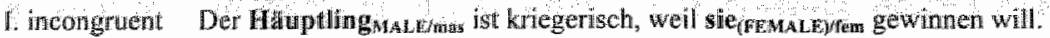

SD T: Thing as wontectert

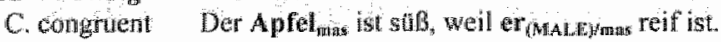

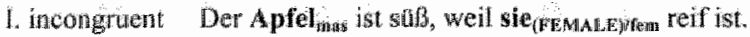

\section{LD P: Person as antecedent}

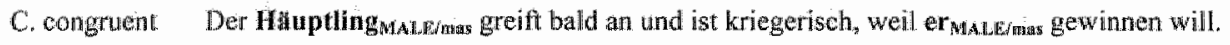

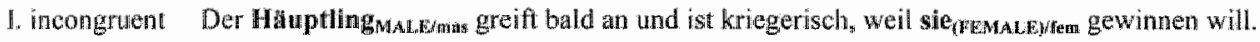

\section{T" Thing as amtecedent}

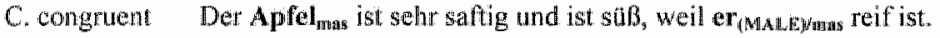

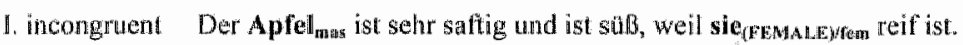

Table 6.1 Example Materials for the experiment Translation: SD P: The chief is martal because he/she wants to win. SD T: The apple is sweet because he/she (it) is ripe. LD P. The chief attacks soon and is martial becase he/she wants to win. $L D$ T: The apple is very jutcy and is sweet because he/she (it) is ripe. FEMALEMALE = biological gender, mas = syntactio gender masculine, fem = syntactic gender feninine.

In the rest of this chapter, a contrast comparing incongruent to congruent sentences will be labeled as Congruency contrast (incongruent minus congruent sentences), and a contrast comparing LD to SD sentences will be labeled as VWM contrast (LD mimus SD sentences). Following the logic of earlier research as reported above, we propose the subsequent hypothesis: For the comparatively simple SD sentences, we expect an increase of Blood oxygen level-dependent (BOLD) response within the left inferior frontal (IF) regions for Congruency, specifically in BA47/45. In contrast, we expect the more complex $1 \mathrm{D}$ sentences to lead to an increase of BOLD response in a more complex activation pattern for Congruency, viz lef inferior frontal and temporal regions (i.e. the superior temporal gyrus) because these sentences tax more verbal working memory resources. Splitting up these comparisons into the two different antecedent types, we aimed to take further insights into syntactic and semantic/syntactic gender information processing during pronoun resolution. The direct comparison of LD and SD conditions should reveal regions specialized for VWM. Co-reference is supposed to be more difficult for LD compared to SD sentences and may therefore lead to an increase of BOLD response in frontal regions for the VWM contrast. In addition, we expect the involvement of temporal regions (Thierry et al. 2003; Martin and Chao, 2001). More specifically, following Martin and Chao (2001), there should be a 
difference for Person compared to Thing conditions. For the VWM contrast for congruent and/or incongruent Person-sentence, we supposed to find an enlarged BOLD response in anterior regions of the temporal lobe, whereas VWM contrast for congruent and/or incongruent Thing-sentence should lead to an increase in BOLD response in more posterior regions.

\subsection{Methods}

\section{Subjects}

Six German native speakers (three females, mean age $24+/-3.4$ ) volunteered to take part in the experiment with written consent and were paid for their participation. All had normal vision, were right-handed and neurological healthy.

\section{Measurements}

Functional measurement: BOLD dependent functional magnetic resonance images were obtained using a 3 Tesla Siemens Magnetom Trio Vision system (Siemens, Erlangen), with echo planar imaging capabilities and a radiofrequency (RF) head (8-)coil (gradient echo EPI, $\mathrm{TR}=2 \mathrm{~s}, \mathrm{TE}=30 \mathrm{~ms}, \mathrm{FOV}=220 \times 220 \mathrm{~mm}^{2}$, flip angle $=80^{\circ}$, matrix size $=64 \times 64$, inplane resolution $3.4375 \times 3.4375 \mathrm{~mm}^{2}$, slice thickness $=3.5 \mathrm{~mm}$, interslice gap $0.35,30$ slices oriented parallel to the AC-PC-line, specified with a midsaggital scout image). One functional run consisted of 550 volumes. In order to avoid a T1 saturation effect we did not present any material during the first 7 volumes and excluded the first four volumes from further analyses. Anatomical measurement: A high-resolution anatomical image was acquired (MP-RAGE, Tweighted, gradient-echo pulse sequence, $\mathrm{TR}=1800 \mathrm{~ms}, \mathrm{TE}=3.44 \mathrm{~ms}$, flip angle $=7^{\circ}, \mathrm{FOV}=$ $256 \mathrm{~mm}$, matrix size $=256 \times 256,192$ sagittal slices, in-plane resolution $1 \times 1 \mathrm{~mm}^{2}$, slice thickness $=1 \mathrm{~mm}$ ).

\section{Material and design}

Eight types of sentences were presented to the subjects as summarized in Table 6.1. We used 64 sentences per condition of the material used in the experiment described in chapter five. Each sentence had two clauses. The first clause in each sentence was the main clause, which described a state of a person or a thing. The person or the thing was the subject of the main clause, and formed the antecedent of the pronoun following later. Care was taken to guarantee equal word frequencies for persons and things using the CELEX-database (Baayen et al., 
1995). The second clause was a subordinate clause introduced by the conjunction well (because. This conjunction was followed by the critical word, a pronoun referring to the person or the thing. All sentences were then copied and the congruent pronoun was replaced by an incongruent pronoun. The just described sentences were the Short Distance (SD) conditions in this experiment. To manipulate VWM in pronoun processing the distance between antecedent and pronoun was increased by adding four words after the antecedent in the given sentences resulting in 512 different sentences in total. Altogether this resulted in a 2 $\times 2 \times 2$ design with VWM (Short Distance/Long Distance SD/LD), antecedent type (person/thing $\mathrm{P} / \mathrm{T}$ ), and congruency (congruent/incongruent $\mathrm{C} / 1$ ) as factors. To minimize repetition, the sertences were distributed across two different lists, counterbalancing antecedent type, congruency, and VWM. Sentences on a list were then pseudo-randomized over four blocks (eight sentences per condition in one run), in such a way that repetitions of antecedents were kept apart as far as possible. We measured three subjects per list. They were pooled again later for analysis.

\section{Procedure}

Subjects were asked to read the sentences concentrated and carefully, as if they were supposed to answer questions concerning the content of the sentences. They should fixate on the screen and avoid possible movements during scanning. Before subjects entered the scanner"s room they performed a training sequence, in which they read seven sentences similar to the experimental ones. Words were white and presented on black background.

The beginning of a trial was the first word of a sentence, which was time-triggered to the sixth man trigger of the scanner following the preceding sentence-trial, thus always starting with a volume scan. In between sentences, an asterisk was presented as fixation point. Thus, the experiment was a slow event-related design, allowing the BOLD response to settle down to baseline in between trials (i.e. sentences in this case). We employed a word-by-word presentation in order to avoid eye-movements associated with free-field sentence reading and to monitor sentence-processing time more carefully. Each word was presented for $350 \mathrm{~ms}$ with a $250 \mathrm{~ms}$ inter-stimulus interval. A word with a period was the sentences ending word. Each scanning session started with a scout imaging for position information. Right after that, two functional scans (550 volumes, 64 sentences, eight sentences per condition) were performed followed by the structural scan allowing subjects to rest. After this break, the remaining two functional runs were performed. 


\section{Image analysis}

Image analysis was performed using BrainVoyager $\mathrm{QX}$ software. For analysis all images were corrected for motion artifacts, corrected for slice-scan time order, temporally smoothed based on a linear trend removal procedure using an lingh pass filter of 3 cycles per time-point, co-registered with the subjects corresponding anatomical (T/-weighted) images, normalized into standard coordinate system (Talairach and Tournoux, 1988), and spatially smoothed using a $4 \mathrm{~mm}$ full-width-at-half-maximum Gaussian kernel. Furthermore, surface rendering, and cortex reconstruction were performed.

For multiple regression analysis of the functional data, a general linear model (GLM) with predictors for (1) general conditions (SD congruent; SD incongruent; $L D$ congruent and $\mathrm{LD}$ incongruent including person and thing antecedents) and for (2) each experimental condition (separating person and thing antecedents) was computed. Using contrast (t) maps, significant differences between conditions were assessed. Multiple comparisons were controlled by the False Discovery Rate (FDR) approach at a level of $\mathrm{q}=0.05$ (Genovese et al., 2002), operating simultaneously on all voxelwise test statistics to determine which tests should be considered statistically significant, and controlling the expected proportion of the rejected hypotheses that are falsely rejected. Reported activations are based on a fixed effects group statistics.

In order to increase the sensitivity of statistical analysis, an improved alignment of the brains was achieved by inter-subject alignment procedure. A polygon mesh of each cortical hemisphere was obtained by segmenting and tessellating the white/grey matter boundary (Dale \& Sereno, 1993; Fischl et al., 1999; Kriegeskorte \& Goebel, 2001). Then, cortical timecourses were created by sampling the functional time-series at positions cotresponding to the nodes of the cortical mesh. Polygon meshes (and corresponding cortical time-courses) were morphed into alignment using spherical representations of the cortical sheet (Fischl, Sereno, Tootell \& Dale, 1999). This procedure provides accurate matching of morphologically homologous cortical locations among subjects while minimizing metric distortion. Again, for multiple regression analysis of the functional data, a general linear model (GLM) approach with predictors for each experimental condition was computed. Using contrast (t) maps, significant differences between conditions were assessed. For thresholding, the equivalent tvalues to the FDR analysis were chosen. We display our averaged functional activation on the hemispheres of one subject, the target brain for the surface-based morphing procedure. This allowed viewing functional activity in relation to sulcal and gyral folds. 


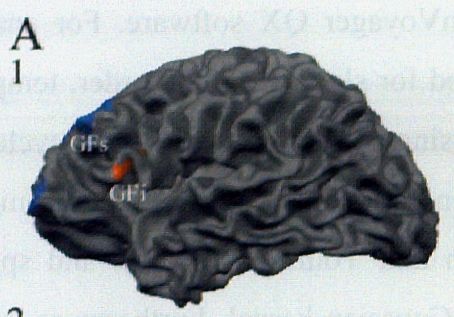

2
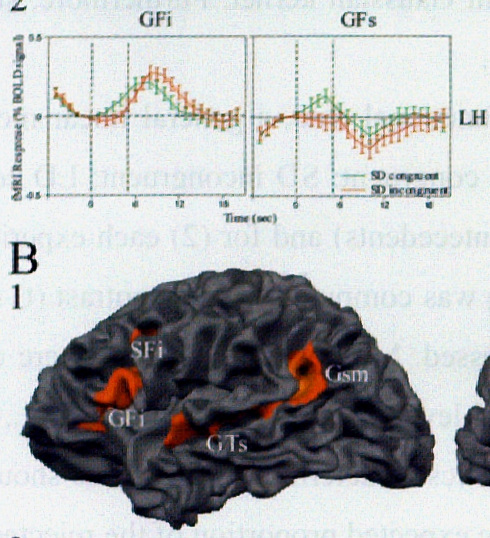

2

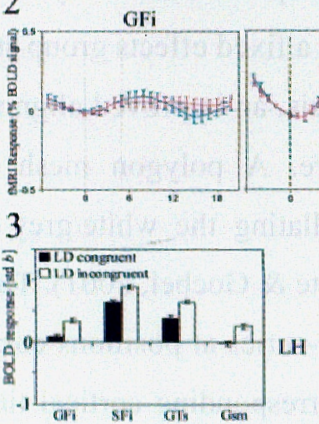

SFi

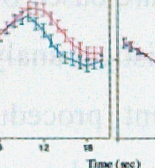

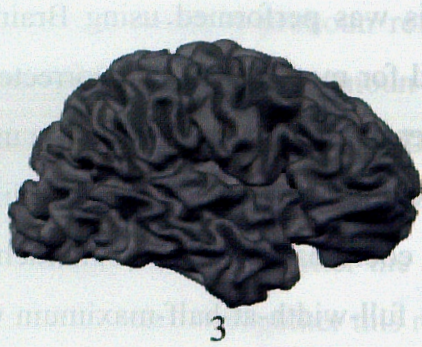

3

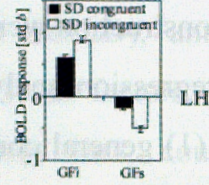

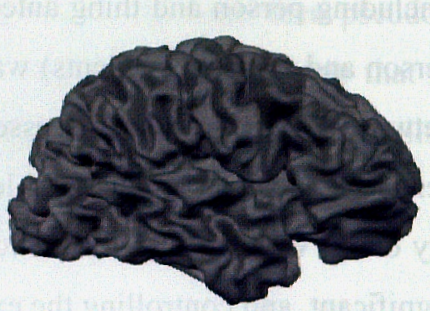

GTs

Gsm

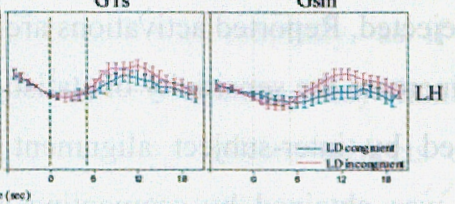


Interaction of gender information and working memory (fMRI)

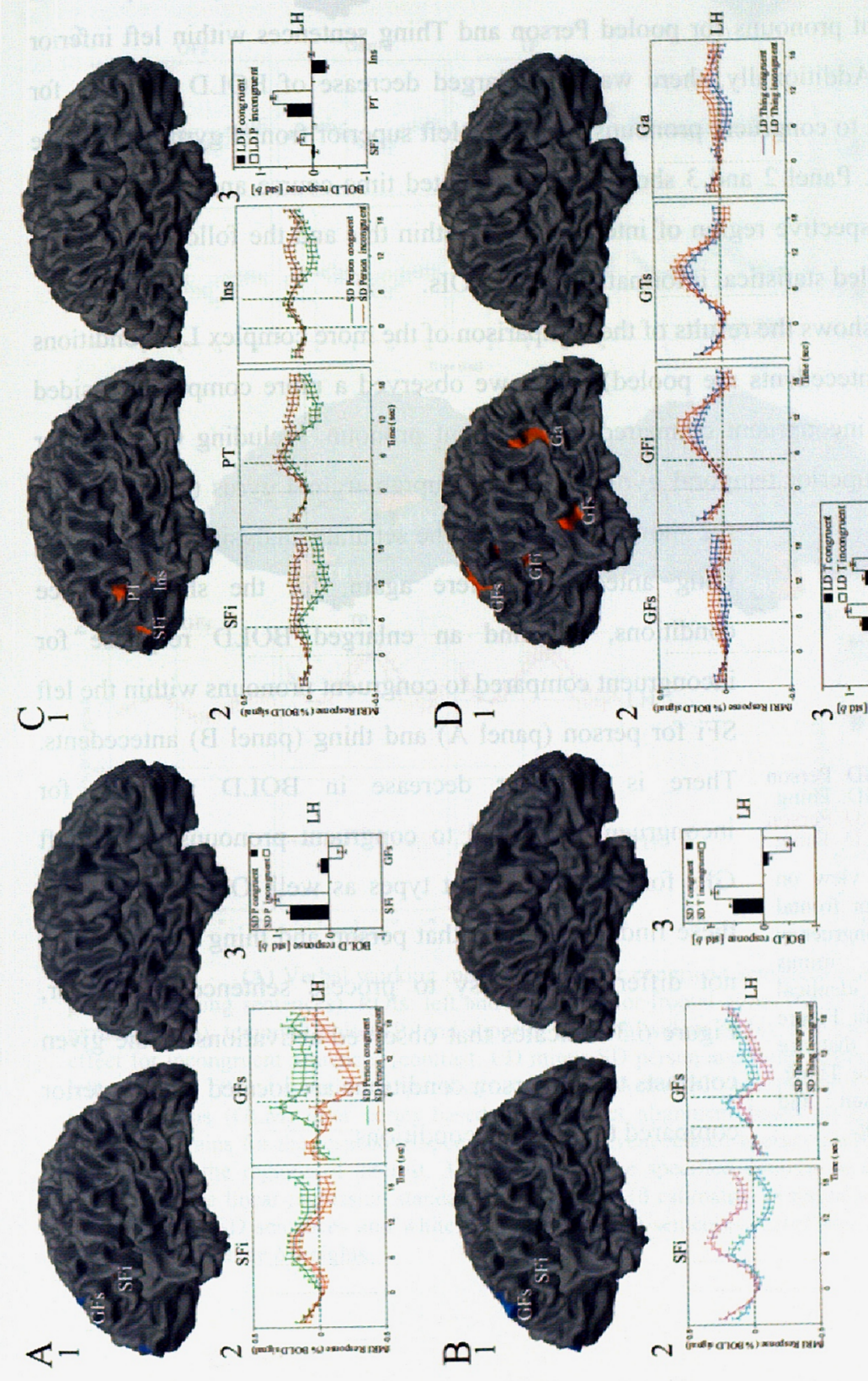

물 을

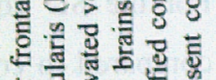

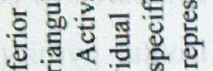

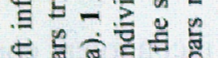

음

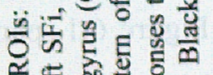

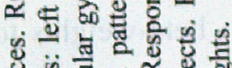

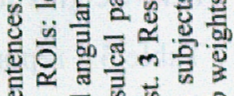

फ मे है है है

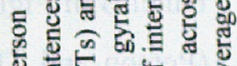

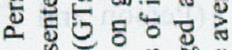

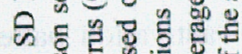

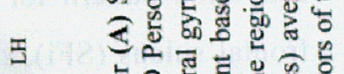

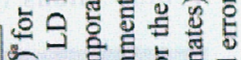

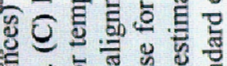

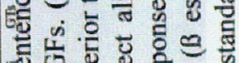

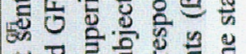

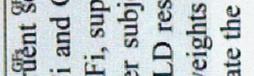

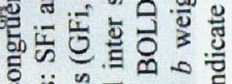

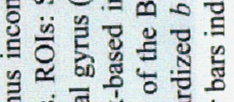

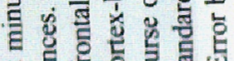
= 온 85 氙

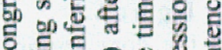

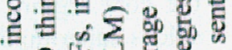

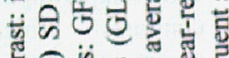

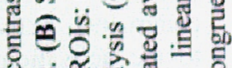
8ำ 중

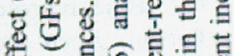

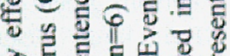

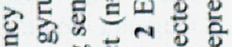

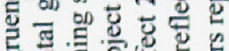

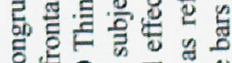

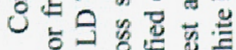
包它焉 है तै के है क

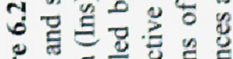

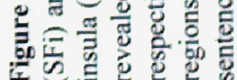




\subsection{Results}

\section{Congruency}

For the SD conditions, we found an enlarged BOLD response for incongruent pronouns compared to congruent pronouns for pooled Person and Thing sentences within left inferior frontal gyrus (GFi). Additionally, there was an enlarged decrease of BOLD response for incongruent compared to congruent pronouns within the left superior frontal gyrus (GFs) (see Figure 6.1, panel A1). Panel 2 and 3 show the event-related time-course and corresponding beta-weights to the respective region of interest (ROI) within this and the following figures. Table 6.2 reports detailed statistical information to the ROIs.

Panel B of Figure 6.1 shows the results of the comparison of the more complex LD-conditions (Person and Things antecedents are pooled). Here, we observed a more complex left-sided activation pattern for incongruent compared to congruent pronoun, including GFi, inferior frontal sulcus (SFi), superior temporal gyrus (GTs) and supramarginal gyrus (Gsm). Figure

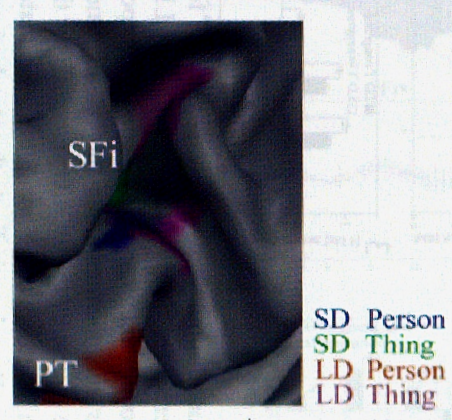

Figure 6.3 Enlarged view on the core region, the inferior frontal sulcus superimposing all congruency contrasts (incongruent minus congruent). Activations are identical to the activations shown in Figure 6.2. Blue represents short distance Person, green short distance Thing, red long distance Person and magenta long distance Thing.
6.2 shows the results to the separate analysis for person and thing antecedents. Here again, for the short distance conditions, we find an enlarged BOLD response for incongruent compared to congruent pronouns within the left SFi for person (panel A) and thing (panel B) antecedents. There is a larger decrease in BOLD response for incongruent compared to congruent pronouns within left GFs for both antecedent types as well. On a first glance, these findings indicate that person and thing conditions do not differ within easy to process sentences. However, Figure 6.3 indicates that observed activations of the given contrasts to SD person conditions are located more anterior compared to SD thing conditions. 


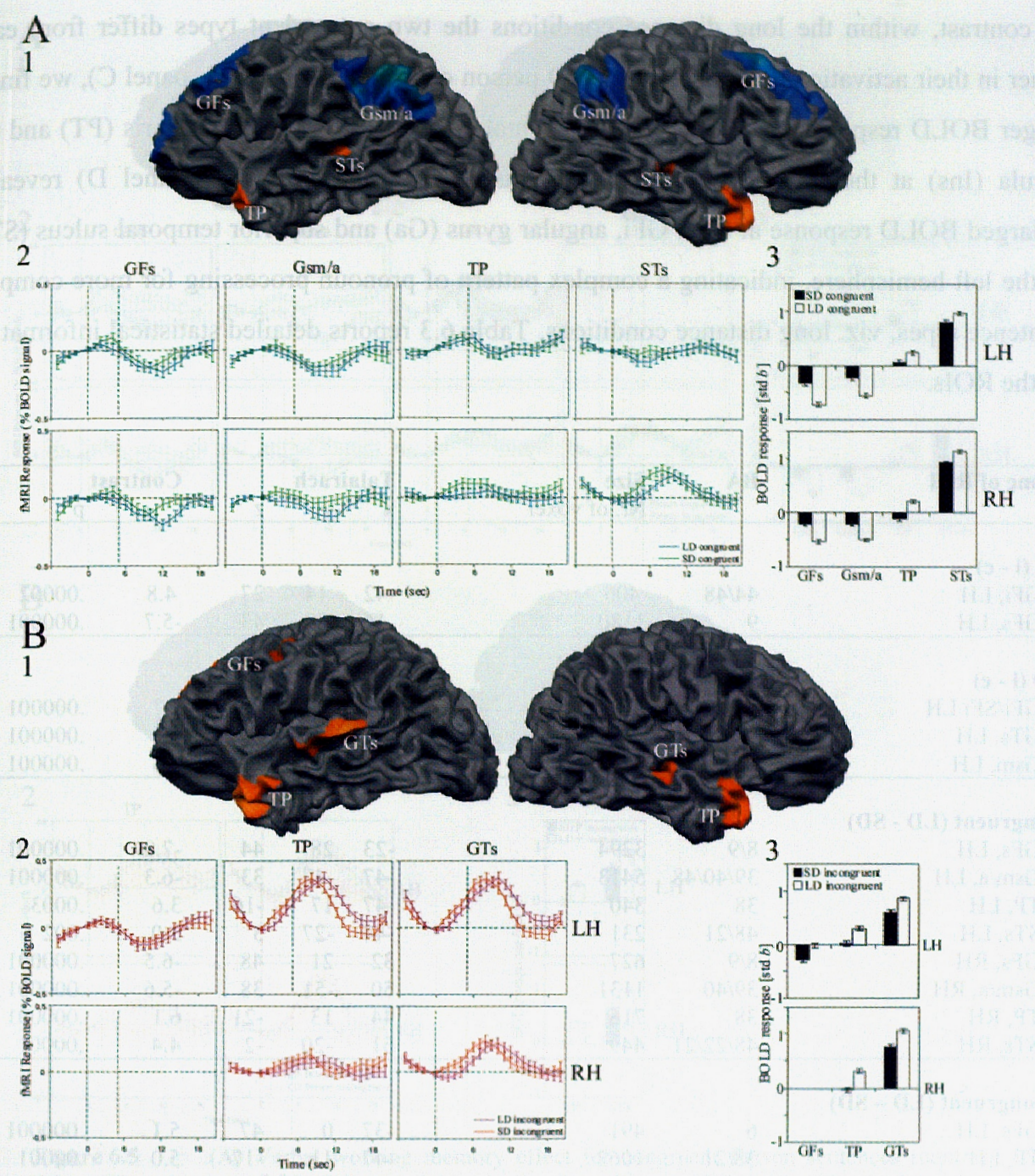

Figure 6.4 (A) Verbal working memory effect for congruent sentences (contrast: LD minus SD persion and thing sentences). ROIs: left and right superior frontal gyrus (GFs), supramarginal/angular gyrus (Gsm/a), temporal pole (TP) and superior temporal sulcus (STs). (B) Verbal working memory effect for incongruent sentences (contrast: LD minus SD person and thing sentences). ROls: left GFs, left and right TP and superior temporal gyrus (GTs). 1 Activated voxels as revealed by across subject $(n=6)$ analysis (GLM) after cortex-based inter subject alignment based on gyral/sulcal pattern of individual brains for the respective specified effect 2 Event-related average time course of the BOLD response for the regions of interest. 3 Responses to the specified contrast in regions of interest as reffected in the linear-regression standardized $b$ weights ( $B$ estimates) averaged across subjects. Black bars represent SD sentences and white bars represent LD sentences. Error bars indicate the standard errors of the average $b$ weights. 
In contrast, within the long distance conditions the two antecedent types differ from each other in their activation patterns. For the LD person condition (Figure 6.2, panel C), we find a larger BOLD response within the inferior frontal sulcus (SFi), pars triangularis (PT) and the insula (Ins) at the left hemisphere. In contrast, LD thing conditions (panel D) revealed enlarged BOLD response at GFs, GFi, angular gyrus (Ga) and superior temporal sulcus (STs) at the left hemisphere, indicating a complex pattern of pronoun processing for more complex sentence types, viz. long distance conditions. Table 6.3 reports detailed statistical information to the ROIs.

\begin{tabular}{|c|c|c|c|c|c|c|c|}
\hline \multirow[t]{2}{*}{ Name of ROI } & \multirow[t]{2}{*}{ BA } & \multirow{2}{*}{$\begin{array}{l}\text { Size } \\
\text { Nr. of voxel }\end{array}$} & \multicolumn{3}{|c|}{ Talairach } & \multicolumn{2}{|c|}{ Contrast } \\
\hline & & & $\mathrm{x}$ & $\mathrm{y}$ & 2 & $t$ & $\mathrm{p}$ \\
\hline \multicolumn{8}{|l|}{$\operatorname{SD}(i-c)$} \\
\hline 1. GFi, LH & $44 / 48$ & 406 & -42 & 14 & 27 & 4.8 & .00002 \\
\hline 2. GFs, $\mathrm{LH}$ & 9 & 1180 & -16 & 38 & 43 & -5.7 & .000001 \\
\hline \multicolumn{8}{|l|}{$L D(i-c)$} \\
\hline 1. GFi/SFi LH & $44 / 48$ & 2250 & -41 & 19 & 19 & 5.7 & .000001 \\
\hline 2. GTs, LH & $22 / 48$ & 1008 & -52 & -15 & 2 & 5.8 & .000001 \\
\hline 3. Gsm, LH & $21 / 22 / 41$ & 1576 & -46 & -47 & 20 & 6.3 & .000001 \\
\hline \multicolumn{8}{|c|}{ Congruent (LD - SD) } \\
\hline 1. GFs, LH & $8 / 9$ & 3294 & -23 & 28 & 44 & -7.8 & .000001 \\
\hline 2. Gsm/a, LH & $39 / 40 / 48$ & 5413 & -47 & -47 & 33 & -6.3 & .000001 \\
\hline 3. TP, LH & 38 & 340 & -47 & 17 & -16 & 3.6 & .0003 \\
\hline 4. STs, LH & $48 / 21$ & 231 & -44 & -27 & 3 & 3.0 & .002 \\
\hline 5. GFs, RH & $8 / 9$ & 627 & 32 & 21 & 48 & -6.5 & .000001 \\
\hline 6. Gsm/a, RH & $39 / 40$ & 1131 & 50 & -51 & 38 & -5.6 & .000001 \\
\hline 7. TP, RH & 38 & 716 & 44 & 13 & -21 & 6.1 & .000001 \\
\hline 8. STs, RH & $48 / 22 / 21$ & 449 & 51 & -20 & -2 & 4.4 & .00001 \\
\hline \multicolumn{8}{|c|}{ incongruent (LD - SD) } \\
\hline 1. GFs, LH & 6 & 491 & -37 & 0 & 47 & 5.1 & .000001 \\
\hline 2. TP, LH & $38 / 21$ & 1062 & -47 & 11 & -17 & 5.0 & .00001 \\
\hline 3. GTs, LH & $22 / 21$ & 1455 & -56 & -26 & 6 & 5.0 & .00001 \\
\hline 4. TP, RH & 38 & 1151 & 46 & 12 & -19 & 6.6 & .000001 \\
\hline 5. GTs, RH & $48 / 22 / 21$ & 746 & 49 & -18 & -4 & 5.7 & .000001 \\
\hline
\end{tabular}

Table 6.2 ROI details for main effects. Activated areas from the fMIRI experiment. All regions reported are listed here and provided with presumed Brodman areas (BA); voxel size; Talairach coordinates as defined by Talairach and Tournoux (1998; $\mathrm{x}$; left-right; $\mathrm{y}$ : anterior-posterior origin in anterior commisure; $\mathrm{z}$ : inferiorsuperior) and statistic values for specific contrasts (t, p). Abbreviations: LH: left hemisphere; RH: right hemisphere; GFi, inferior frontal gyrus; GFs: superior frontal sulcus; SFi: inferior frontal sulcus; GTs: superior temporal gyrus; Gsm: supramarginal gyrus; Ga: angular gyrus; TP: temporal pole; STs; superior temporal sulcus. 

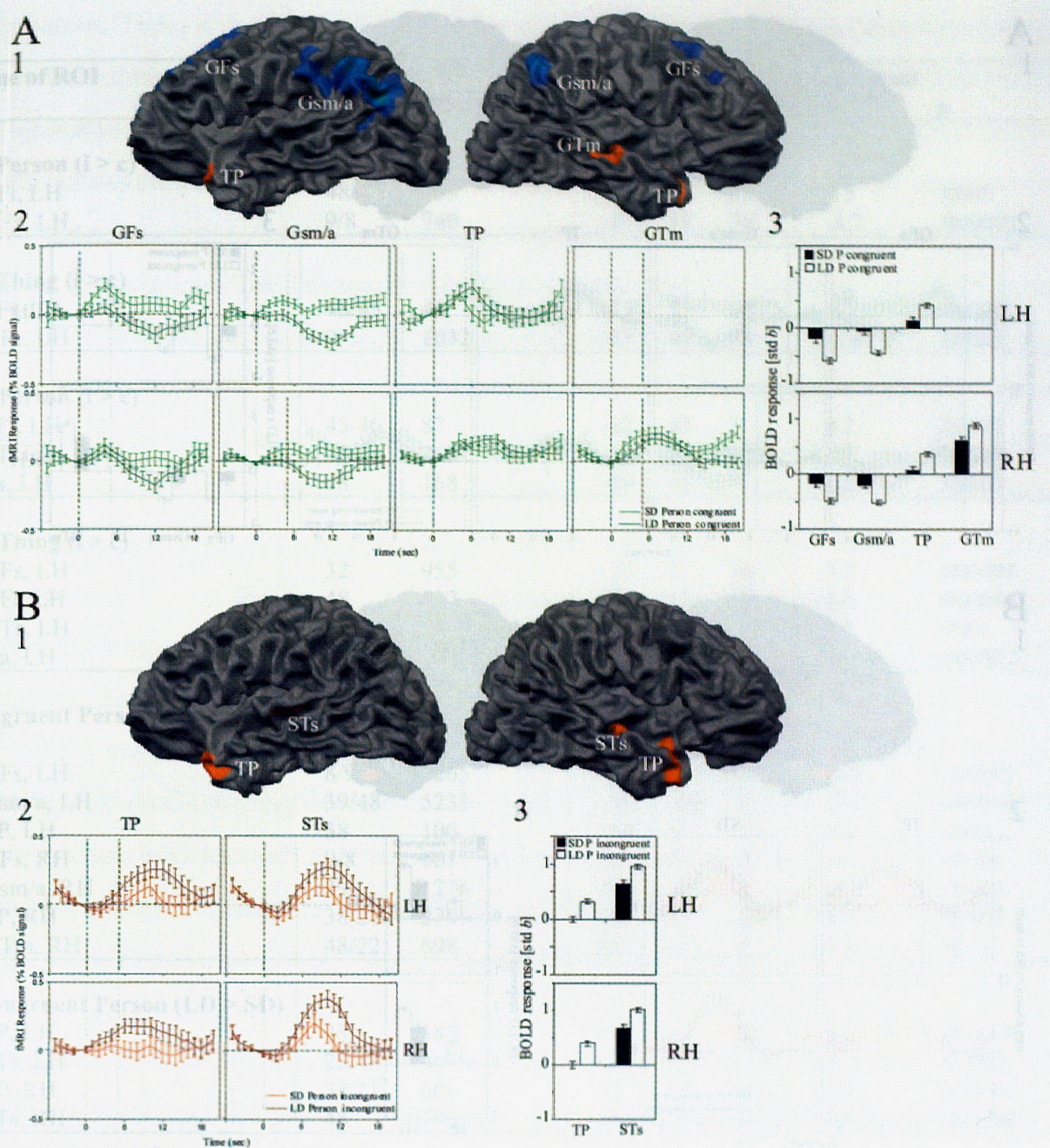

Figure 6.5 (A) Verbal working memory effect for congruent Person sentences (contrast: LD minus SD sentences). ROIs: left and right superior frontal gyrus (GFs), supramarginal/angular gyrus (Gsm/a), temporal pole (TP) and right medial temporal gyrus (GTm). (B) Verbal working memory effect for incongruent Person sentences (contrast: LD minus sentences). ROls: left and right TP and superior temporal sulcus (STs). 1 Activated voxels as revealed by across subject $(n=6)$ analysis (GLM) after cortex-based inter subject alignment based on gyral/sulcal pattern of individual brains for the respective specified effect 2 Event-related average time course of the BOLD response for the regions of interest. 3 Responses to the specified contrast in regions of interest as reflected in the linear-regression standardized $b$ weights ( $\beta$ estimates) averaged across subjects. Black bars represent SD sentences and white bars represent LD sentences. Error bars indicate the standard errors of the average $b$ weights. 

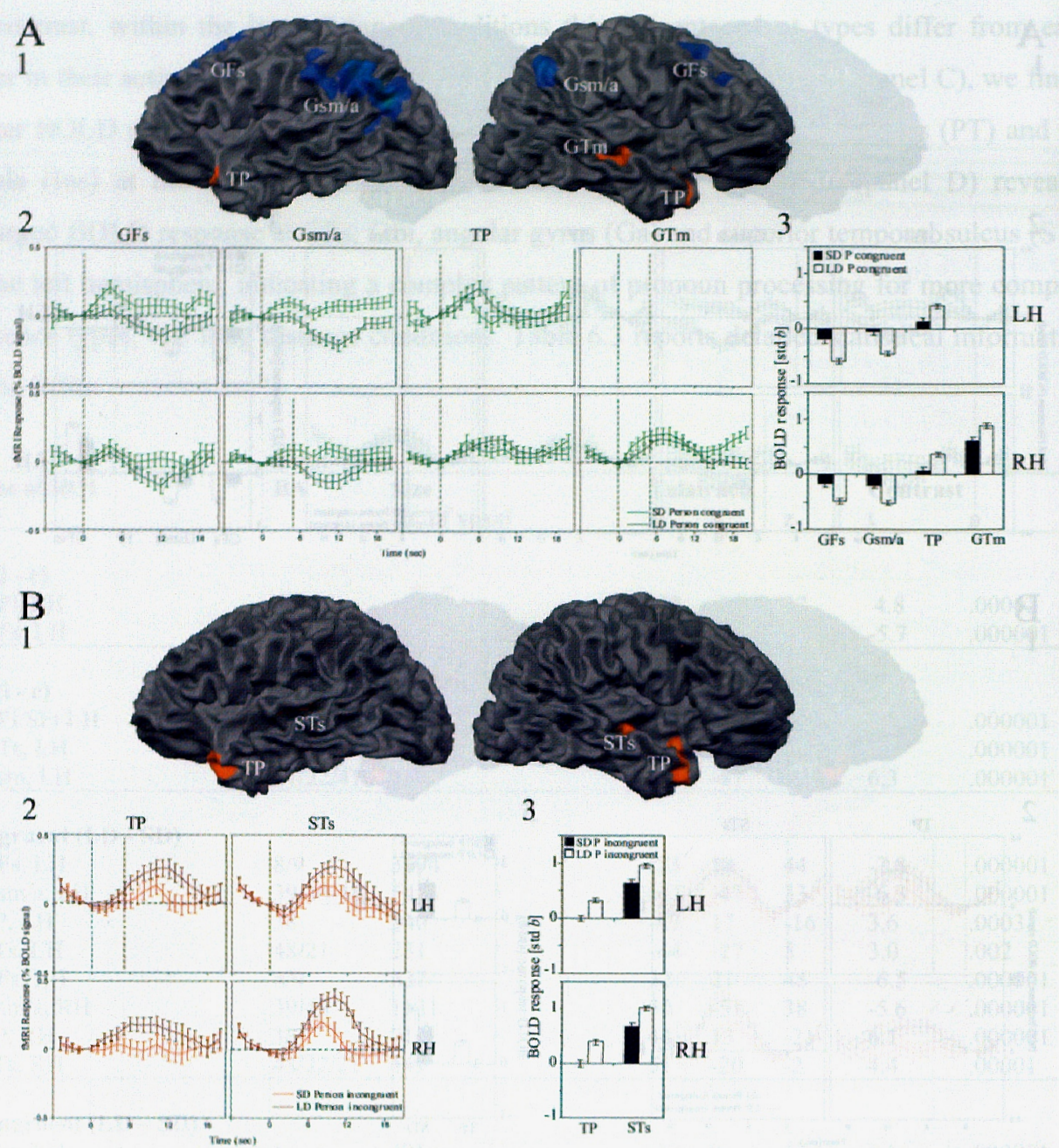

Figure 6.6 (A) Verbal working memory effect for congruent Thing sentences (contrast: LD minus SD sentences). ROIs: left and right superior frontal gyrus (GFs), left angular gyrus (Ga) and right temporal pole (TP). (B) Verbal working memory effect for incongruent Thing sentences (contrast: LD minus sentences). ROIs: left GFs and superior temporal gyrus (GTs) and right TP and medial temporal sulcus (STm). 1 Activated voxels as revealed by across subject $(n=6)$ analysis (GLM) after cortex-based inter subject alignment based on gyral/sulcal pattern of individual brains for the respective specified effect 2 Event-related average time course of the BOLD response for the regions of interest. 3 Responses to the specified contrast in regions of interest as reflected in the linear-regression standardized $b$ weights ( $\beta$ estimates) averaged across subjects. Black bars represent SD sentences and white bars represent LD sentences. Error bars indicate the standard errors of the average $b$ weights. 


\begin{tabular}{|c|c|c|c|c|c|c|c|}
\hline \multirow[t]{2}{*}{ Name of ROI } & \multirow[t]{2}{*}{ BA } & \multirow{2}{*}{$\begin{array}{l}\text { Size } \\
\text { Nr. of voxel }\end{array}$} & \multicolumn{2}{|c|}{ Talairach } & \multirow{2}{*}{\multicolumn{2}{|c|}{$\begin{array}{ll} & \text { Contrast } \\
\mathrm{z} & \mathrm{t}\end{array}$}} & \multirow{2}{*}{$p$} \\
\hline & & & $\mathrm{x}$ & y & & & \\
\hline \multicolumn{8}{|c|}{ SD Person $(i>c)$} \\
\hline 1. SFi, LH & $48 / 44$ & 109 & -43 & 15 & 25 & 3.5 & .0005 \\
\hline 2. GFs, LH & $9 / 8$ & 740 & -17 & 38 & 50 & -4.7 & .000003 \\
\hline \multicolumn{8}{|c|}{ SD Thing $(i>c)$} \\
\hline 1. SFi, LH & $48 / 45$ & 46 & -47 & 23 & 20 & 4.3 & .000001 \\
\hline 2. GFs, LH & 9 & 1032 & -27 & 40 & 38 & -5.0 & .00002 \\
\hline \multicolumn{8}{|c|}{ LD Person $(i>c)$} \\
\hline 1. SFi, LH & $45 / 46$ & 87 & -42 & 43 & 5 & 4.2 & .00003 \\
\hline 2. PT, LH & $45 / 48$ & 263 & -44 & 22 & 12 & 4.1 & .00004 \\
\hline 3.Ins, LH & 48 & 168 & -29 & 16 & -1 & 4.2 & .00003 \\
\hline \multicolumn{8}{|c|}{ LD Thing $(i>c)$} \\
\hline 1. GFs, LH & 32 & 955 & -10 & 27 & 50 & 5.2 & .000001 \\
\hline 2. GFi, LH & 48 & 537 & -41 & 12 & 30 & 4.6 & .000004 \\
\hline 3. GTs, LH & 48 & 226 & -48 & -12 & -1 & 3.9 & .0001 \\
\hline 4. $\mathrm{Ga}, \mathrm{LH}$ & $41 / 21$ & 923 & -45 & -46 & 20 & 4.7 & .000002 \\
\hline
\end{tabular}

Congruent Person (LD > SD)

\begin{tabular}{llllllll} 
1. GFs, LH & $8 / 9$ & 1265 & -22 & 24 & 46 & -5.3 & .000001 \\
2. Gsm/a, LH & $39 / 48$ & 5233 & -45 & -50 & 32 & -5.3 & .000001 \\
3. TP, LH & 38 & 100 & -48 & 18 & -15 & 3.6 & .0003 \\
4. GFs, RH & $9 / 8$ & 661 & 36 & 21 & 45 & -4.3 & .00001 \\
5. Gsm/a, RH & 39 & 1276 & 49 & -52 & 35 & -4.2 & .00002 \\
6. TP, RH & $38 / 21$ & 274 & 44 & 11 & -20 & 4.0 & .00007 \\
7. GTm, RH & $48 / 22$ & 698 & 49 & -17 & -5 & 3.8 & .0001 \\
\hline
\end{tabular}

\begin{tabular}{llllllll} 
Incongruent Person (LD > SD) & & & & & & \\
1. TP, LH & 38 & 380 & -44 & 15 & -21 & 4.5 & .000008 \\
2. STs, LH & $22 / 48$ & 367 & -54 & -25 & 5 & 4.2 & .00002 \\
3. TP, RH & $38 / 21$ & 666 & 47 & 11 & -19 & 5.5 & .000001 \\
5. STs, RH & 48 & 798 & 49 & -17 & -4 & 4.8 & .000002 \\
\hline & & & & & & & \\
Congruent Thing (LD > SD) & & & & & & & \\
1. GFs, LH & $9 / 8$ & 1763 & -23 & 30 & 43 & -5.7 & .0000001 \\
2. Ga, LH & $39 / 40$ & 890 & -49 & -49 & 36 & -3.9 & .00009 \\
3. GFs, RH & $8 / 9$ & 201 & 33 & 23 & 48 & -4.5 & .000006 \\
4. TP, RH & 38 & 241 & 42 & 12 & -25 & 4.9 & .000001 \\
\hline & & & & & & & \\
Incongruent Thing (LD > SD) & & & & & & & \\
1. GTs, LH & 22 & 204 & -60 & -33 & 10 & 4.4 & .00002 \\
2. GFs, LH & 9 & 1015 & -8 & 44 & 42 & 4.2 & .00002 \\
3. TP, RH & 38 & 498 & 43 & 14 & -20 & 5.5 & .000001 \\
4. STm, RH & $21 / 20$ & 176 & 49 & -5 & -20 & 5.0 & .000001 \\
\hline
\end{tabular}

Table 6.3 ROI details for Person and Thing. Activated areas from the fMRI experimemt. All regions reported are listed here and provided with presumed Brodman areas (BA); voxel size; Talairach coordinates as defined by Talairach and Tournoux $(1998 ; \mathrm{x}$ : left-right; $\mathrm{y}$ : anterior-posterior origin in anterior commisure; $\mathrm{z}$ : inferior-superior) and statistic values for specific contrasts (t, p). Abbreviations: LH: left hemisphere; RH: right hemisphere; SFi: inferior frontal sulcus; GFs: superior frontal sulcus; PT: pars triangularis; Ins: insula; GFi, inferior frontal gyrus; GTs: superior temporal gyrus; Ga: angular gyrus; Gsm: supramarginal gyrus; TP: temporal pole; GTm: medial temporal gyrus; STs: superior temporal sulcus; STm: medial temporal sulcus. 


\section{Verbal Working Mewory}

Comparing the congruent comparative complex sentences (LD) to the congruent relative easy sentences (SD) (Figure 6.4, panel A), we found a bilateral activation pattern including four different areas. Within left and right temporal pole (TP) and superior temporal sulcus (STs), congruent $L D$ sentences revealed an enlarged increase in BOLD response compared to congruent SD sentences. In contrast, with the GFs and supramarginal/angular gyrus (Gsm/a) we found the opposite direction of the effect; congruent LD sentences showed an enlarged decrease of BOLD response compared to congruent SD sentences.

Interestingly, comparing incongruent LD to SD conditions (Panel B) we find a different activation pattern, including the left GFs and bilaterally TP and GTs. In addition, there seemed to be a delay of hemodynamic response for incongruent LD sentences compared to incongruent SD sentences in left temporal regions (panel B2).

Now, separating person and thing antecedents for the VWM contrasts, we found the following results for Person (Figure 6.5) and for Thing antecedents (Figure 6.6). Comparing congruent LD to SD conditions of Person antecedents (panel A), we found an enlarged increase of BOLD response for bilateral TP and the right medial temporal gyrus (GTm). Here as well, there was an enlarged decrease of the hemodynamic function in bilateral GFs and Gsm/a for congruent LD compared to SD sentences. In contrast, incongruent Person (panel B) antecedents revealed an increase of BOLD response for LD compared to SD conditions within bilateral temporal regions, viz. TP and ST'S.

Comparing congruent LD to SD sentences about thing antecedents (Figure 6.6, panel A), we found an enlarged decrease of hemodynamic response at bilateral GF's and left Ga, and a larger increase of hemodynamic response at right TP. In contrast, incongruent thing sentences (panel BI) revealed an increase of BOLD response at left GFs and GTs, and right STm and TP for $\mathrm{LD}$ compared to SD sentences. For this contrast no difference in the event-related BOLD response was observed (panel B2).

\subsection{Discussion}

The aim of this study was to investigate the involvement of semantic/biological and syntactic gender information processing and possible interaction with verbal working memory during the process of building up co-reference between a pronoun and person- or thing antecedents in German. Persons are characterized by semantic/biological and accompanying syntactic gender 
information. Thus, a pronoun referring back to a person may be re-referenced by biological and/or syntactic gender information. In contrast, in German things are only characterized by an (arbitrary) syntactic gender information and so building up co-reference between pronoun and thing may solely be based on syntactic gender information. To manipulate verbal working memory, we increased the distance between antecedent and pronoun. First, we discuss the findings to Short- and Long Distance conditions for congruent and incongruent pronouns and then specify these in more detail by means of Person- and Thing antecedents (Congruency effects). Later on, we focus on verbal working memory by comparing congruent LD with SD sentences and incongruent LD with SD sentences and specify these as well in more detail by means of Person- and Thing antecedents.

\section{Congruency}

For incongruent compared to congruent sentences within easy to solve SD sentences we found an enlarged BOLD signal change within the left GFi, indicating that the left GFi is the core region for syntactic and semantic gender information processing during the process of building up co-reference between a pronoun and belonging antecedent. The activation of GFi is in accordance with earlier studies which report the involvement of that region for syntactic and semantic information processing (Newman et al., 2001, Röder et al., 2002, Friederici, 2003). Surprisingly, we found significantly less activation for incongruent compared to congruent conditions in left superior frontal regions (blue colored areas in Figure 6.1). Looking into the time course of the BOLD signal change, we see an enlarged decrease of BOLD signal change for incongruent compared to congruent SD sentences in these regions. What does a negative BOLD response mean? Stefanovic, Warnking and Pike (2004) described the negative BOLD response as a marker of netronal deactivation, defined as the hemodynamic down regulation accompanying neuronal inhibition. According to this, our enlarged decrease of BOLD response of incongruent compared to congruent SD sentences suggest a neuronal inhibition within superior frontal areas due to the clear and easy to detect violation of the pronoun's gender information. Noticing that the pronoun cannot be rereferenced to the close antecedent, the comprehension system suppresses further integration attempts indicating a general monitoring rolle of the left GFs. These frontal areas suggest involvement of short term memory in this process. In contrast, the more difficult to process LD condition revealed a different activation pattern for incongruent compared to congruent sentences. Here, we found a left sided activation of inferior frontal regions including GFi and $\mathrm{SFi}$, superior temporal regions (SFi) and the supramarginal gyrus. This complex activation 
pattern suggest that building up co-reference over a longer distance is a different process compared to the SD scenario. In contrast to the SD condition, the LD congruency contrast lacks in prefrontal activation and shows instead a salient left temporal lobe activation. This might reflect an access to long-term memory (temporal regions) instead of a search/access to VWM (more frontally).

Splitting the SD and LD congruency effects into Person and Thing antecedents, we achieved comparable results for the SD sentences for both antecedent types, confirming the key role of GFi in building up co-reference during the process of pronoun resolution based on syntactic and semantic gender information. However, within $L D$ sentences the two antecedent types differed from each other in their activation patterns. As we solely found an enlarged BOLD signal change in left inferior frontal regions ( $\mathrm{SFi}_{4} \mathrm{PT}$ and Ins) for incongruent compared to congruent person sentences we concluded that biological gender accompanied with syntactic gender information processing in complex sentences is processed via or within these regions. In contrast, for $\mathrm{LD}$ thing sentences (pure syntactic gender information processing in more difficult sentences) we observed a complex activation pattern on the left hemisphere for incongruent compared to congruent thing sentences including superior and inferior frontal regions (GFs and GFi) and the angular gyrus. It seens that $L D$ thing sentences are the most difficult to process sentences within this experiment. The comprehension system can only use syntactic gender information to create coherence between pronoun and antecedent. Possibly, the antecedent has been processed such a long time ago that there seems to be no attempt to integrate the incongruent pronoun into the sentence. Instead, there seems to be a search for an alternative antecedent (i.e. a person as potential actor outside the sentence context) indexed by activation of brain regions usually referenced to semantic information processing, viz. the Gsm. This process of searching for an antecedent outside the thing-sentence context is supported by previous event-related potentials data from our own group. The thing-sentence condition elicited a negative effect, the $\mathrm{N} 400$ with incongruent being more negative than congruent, on the word following the pronoun, as reported by Hammer and colleagues (2005, chapter 2 this volume). For the LD person case, we did not find an indication to this external semantic search in temporal long-term memory related cortical regions, as sollely frontal regions were activated. Here, it seems that two types of gender information - biological and syntactic - create a strong coherence between pronoun and antecedent resulting in a successful refusal of the incongruent pronoun. Figure 6.3 showed the engagement of the key region, viz. GFi, for all congmency contrasts. Even though there is a certain amount of overlapping regions, it seems that person antecedents are located more anterionly compared to 
thing antecedents. This finding is in accordance with results by Roder and colleagues (2002), Who reported an interaction of syntactic and semantic processing within left inferior frontal regions. But it also suggests further specifications of the GFi for the processing of different types of antecedents or actors in a sentence.

\section{Verbal Working Memory}

A direct measure of VWM was realized by comparing $L D$ to SD sentences. This was done for congruent and incongruent sentences separately - first pooling Person and Thing antecedents and afterwards separating both antecedent types.

For the pooled approach we found enlarged BOLD signal change for congruent LD sentences compared to SD sentences within bilateral temporal pole (TP) and superior temporal sulcus (STs) and additionally an unexpected decreased BOLD signal change for congruent LD to SD sentences in GFs and Gism/a on both hemispheres. The activation of temporal regions for congruent LD compared to SD sentences indicates that the access to antecedents requires more processing capacity within temporal regions in cases of higher distance between pronoun and antecedent, where an "object representation system" is supposed to be stored (Damasio, 1996; Thierry et al., 2003). In contrast, the decreased BOLD signal change within bilateral GFs and Gsm/a for LD compared to SD sentences containing congruent pronouns remains speculative. Granted the assumption that a decreased BOLD response reflects a neuronal inhibition, these regions were deactivated for congruent $L D$ compared to SD sentences, eventually by processes that are sensitive to detect distances between words, i.e. integrate words into sentence and discourse context over time. On the other hand, as expected, we do find increased activation for congruent $\mathrm{LD}$ sentences compared to $\mathrm{SD}$ sentences in temporal regions indicating access to the temporal semantic working memory system (Martin \& Chao, 2001) in order to re-activate the distant antecedent. Separating congruent sentences into Person and Things antecedents, we found that the described results solely apply to Person sentences. In contrast, for congruent Thing sentences, we found a different activation pattern, with a larger increase of BOLD signal change at right TP and an enlarged decrease at bilateral GFs and left Ga for LD compared to SD sentences. This finding indicates that for pronoun resolution within congruent sentences the neural comprehension system is sensitive to the antecedent in combination with VWM manipulation, reflected in a temporal activation for LD compared to SD Person sentences (biological and syntactic gender information), whereas for the thing case (solely syntactic gender information) no temporal activation was found. 
Now, comparing incongruent $\mathrm{LD}$ to SD sentences we found left-sided activation of the GFs, and a bilateral activation for TP and GTs. In more detail, we found a delayed event-related time-course for $\mathrm{LD}$ compared to $\mathrm{SD}$ sentences within left temporal regions. In general, the localization of the activation pattem is congruent wh the findings by Thierry and colleagues (2003). Turning to incongruent comparisons, Person sentences revealed an increase of BOLD signal change for LD compared to SD conditions within bilateral anterior temporal regions (TP and STs), whereas Thing sentences revealed an increase of BOLD response at left posterior regions (GFs and GTs) and right anterior temporal regions (STm and TP) for LD compared to SD sentences. These findings indicate that temporal regions are sensitive to our experimental manipulation with respect to the type of antecedent of the pronoun, resulting in an anterior temporal activation for Person antecedents and left-sided more posterior temporal activation for Thing antecedents. This is in congruency with the semantic working memory network that was described by Martin and Chao (2001). The authors stated that posterior temporal regions seem to be associated with more general representations, whereas anterior temporal regions are related to more specific representations, i.e. famous faces. We suggest that these areas get active due to a search for an appropriate antecedent in case of violations, but also in case of longer temporal distance between antecedent and pronoun (as in our LD conditions).

\section{Conclusions}

The presented results revealed that building up co-reference between pronoun and antecedent within sentences depends on the distance between pronoun and antecedent in addition to the availability of gender information of the antecedent (syntactic or biological/syntactic). If an incoming pronoun cannot be integrated into a sentence due to an incongruent syntactic or biological type of gender information, left inferior frontal regions become more activated compared to congruent pronouns independent of the distance between pronoun and antecedent. Zooming into that core region showed that sentences about a person are processed more anteriorly compared to sentences about things indicating that this region differentiates between person and thing antecedents during pronoun processing. In addition, if the pronoun is close to the person or thing antecedents as in SD sentences, left superior frontal regions seemed to inhibit further integration attempts, indicated by a decrease of the hemodynamic response function. Thus, a general monitoring processor is in these superior frontal areas, which inhibits further integration attempts in case of close and clear gender violations for both types of antecedents. With an increased distance between antecedent and pronoun (LD 
sentences), this inhibition process was absent. Instead, we observed an additional activation within temporal and temporo-parietal regions for $L D$ thing sentences containing an incongruent compared to a congruent pronoun. The comprehension system can only use syntactic gender information to link the pronoun to the antecedent. Thus, there seems to be a search for an alternative antecedent (i.e. a person as potential actor outside the sentence context) indexed by an activation of brain regions usually referenced to semantic information processing, viz. the Gsm. Furthermore, the neural comprehension system is sensitive to the distance between pronoun and antecedent depending on availability of gender information within congruent sentences, which was reflected in a temporal activation for LD compared to SD Person sentences (biological and syntactic gender information). In contrast, for the thing case (solely syntactic gender information) no temporal activation was found. For incongruent sentences we found a difference for antecedent types within temporal regions, with am anterior temporal activation for Person antecedents and left-sided more posterior temporal activation for Thing antecedents. We suggest that these areas get active due to a search for an appropriate antecedent depending on information availability in case of violations, but also, in case of longer temporal distance between antecedents and pronouns as in our LD case. 
7

Summary / Samenvatting 


\section{Summary}

A multi-method approach was used to explore the cognitive processes of pronoun resolution within sentences in six different experiments. German pronouns (erMALE/misculise: siepremalefiteminine) that refer to a person are determined by the biological gender (MALE/FEMALE) and/or syntactic gender (masculine/feminine) of the person. Pronouns ( $\mathrm{er}_{\text {inatsculine, }}$ sie $_{\text {fenmininis. }}$ ) that refer to a thing are determined by its arbitrary syntactic gender (Garten (garden) masculine, Tasche (hand-bag) feminune). We presented sentences in word-by-word reading paradigms with either a person or a thing as antecedent, which were followed by a congruent or incongruent pronoun. Hereby, it was of interest to investigate whether semantic and/or syntactic integration are involved in establishing coreference between a pronoun and an antecedent by means of gender information violation. In addition, we increased the distance between pronoun and antecedent to elucidate whether syntactic and/or semantic pronoun resolution would interact with verbal working memory (VWM). By using different methods, we were able to investigate the time-course (when) of investigated processes (event rellated potentials, ERP) and the localization (where) of respective neuronal networks (functional magnetic resonance imaging, fMRI).

The review of literature given in Chapter 1 shows present knowledge about the involvement of syntactic and semantic information processing for establishing co-reference between a pronoun and a suitable antecedent and the influence of extra processing due to increased difficulty in sentences. Altogether, the review lead to three important ERP components, namely the N400 (semantic integration problems), the P600 (syntactic processing difficulty and reanalysis) and the left anterior negativity (LAN, working memory). Neuroimaging studies detected a complex neuronal network for language comprehension including i.e. inferior frontal regions, superior and middle temporal regions, and regions adjacent to the supramarginal/angular gyrus.

In Chapter 2 we present ERP findings which show that violations are reflected in the P600 in pure syntactic gender violation (things) and in combination of syntactic and biological gender violation (persons) at pronoun position. This effect was larger for the person compared to the thing condition suggesting that the P600 is sensitive to the availlability of semantic gender information of the antecedent (here, whether or not it has biological gender). Furthernore, the observed N400 for the word following the pronoun in case of thing but not 
person antecedent types indicated that this effect reflects continuous integration processes for things, whereas for persons the integration seems to be finished at pronoun position.

Surprisingly, contrary to the clear results of the first experiment, simultaneously recorded magnetoencephalographic data failed to reveal reliable differences between incongruent and congruent pronouns referring back to person or thing antecedents as reported in Chapter 3 . This result is discussed as demonstrating different outcomes for ERP and evert related fields (ERF) data due to different characteristics of the two methods, i.e. measuring tangential vs. radial sources or widely distributed sources for higher cognitive processes. When higher processing stages, such as language processing are the interest of investigation, the corresponding sources may be widely distributed and currents flowing simultaneously in opposing walls of a sulcus may partially cancel each other out. In that case, ERP might prove to be the more valuable source of information.

To localize the underlying neuronal networks of biological and syntactic gender integration during pronoun processing, we performed an event-related AMRI study, described in Chapter 4. Overall, syntactic processing activated left hemispheric areas adjacent to Broca's area and superior temporal sulcus (STS), whereas processing of the biological gender information, in addition, involved a bilateral network including inferior frontal gyrus (GFi) and the supramarginal gyrus (Gsm). A comparison with ERPs elicited with identical material (experiment $\mathbb{1}$ in chapter 2) suggested that the information is integrated $400-700 \mathrm{~ms}$ after target onset, visible in both cases as a P600 with different effect sizes. The results illuminate temporal and spatial integration of syntactic and semantic processing during the establishment of co-reference between a pronoun and its referring noun. Pronouns referring back to persons can be matched to the antecedent by biological and syntactic information, requesting more processing capacity indicated by the large P600 and the activation of a bilateral cortical network, including GFi and Gsm/Ga. In contrast, pronouns referring back to things are solely matched by pure syntactic gender information reflected in a smaller P600 indicating less processing activity and an activation of the left GFi and STs.

Integrating a pronoun into a sentence context requires VWM resources because the earlier mentioned antecedent has to be re-activated to link the pronoun to the antecedent. In the experiment described in Chapter 5 we manipulated this process by increasing the distance (long distance conditions, LD) across person and thing sentences and compared these to the 
sentences of the former experiments (short distance conditions, SD) within an ERP experiment. In general, this VWM manipulation effected the N400/P600 patterns of our sentence types, indicating that VWM interacts with semantic and syntactic processing. Furthermore, our data suggest that sentence parsing does not only depend on semantic or syntactic information but it is rather a highly flexible process. The respective N400/P600 patterns suggested that the comprehension system switches preference between semantic and syntactic information access during pronoun processing depending on demands on VWM resources and information availability. In addition, we found an $\mathrm{N} 400$ on the word following the pronoun for SD and $L D$ thing sentences, indicating an ongoing integration process after pronoun position, independent of distance manipulation.

The aim of the AMRI study reported un Chapter 6 was to localize processing of syntactic and semantic gender information and possible interaction with VWM during the process of building up co-reference between a pronoun and its antecedent, using the material as described in chapter five. As a main result, we found that left inferior frontal regions are the core regions for building up co-reference between pronoun and antecedent, with a slight local differentiation dependent on antecedent type. In addition, we suggest that left superior frontal regions may be described as a general monitoring processor for clear gender violations within relatively easy SD sentences, as we found deactivation for incongruent compared to congnuent pronouns within these regions, but not for LD sentences. Instead, we observed additional activation within temporal and temporo-parietal regions if the comprehension system can only rely on syntactic gender information to link the pronoun to a distant antecedent (LD thing). The search for an altemative antecedent was indexed by activation of brain regions usually referenced to semantic information processing, viz. the Gsm. Furthermore, we demonstrated sensitivity to the distance manipulation, depending on availability of gender information within congruent sentences, which was reflected in a temporal activation for LD compared to SD person sentences, whereas for the thing case no temporal activation was found. For incongruent sentences we found an anterior temporal activation for Person antecedents and a more posterior temporal activation for thing antecedents. We suggest that these areas get active due to a search for an appropriate antecedent, depending on information availability in case of violations, but also in case of longer temporal distance (LD) between antecedents and pronouns. 


\section{Samenvatting}

In zes verschillende experimenten werd een multi-methodebenadering gebruikt om de cognitieve verwerkingsprocessen van pronomen bimnen zinnen te onderzoeken. Duitse pronomen die naar een persoon verwijzen (erMAN/mannelijk, sievRouw/wrouwelijk) worden bepaald door het biologische geslacht (MAN/VROUW) en/of het syntactische geslacht van de persoon (mannelijk/vrouwelijk). Pronomen die naar een ding verwijzen ( $\mathrm{er}_{\text {mauntelijk, }}$ sie $e_{\text {wouwwelikk }}$ ) worden bepaald door hun willekeurig syntactische geslacht (Garten (tuin) mannelijk, Tasche (handtas) vrouwelijk). In woord-voor-woord lees paradigma's boden wij zinnen aan met een persoon of een ding als antecedent die door een congruent of incongruent pronoom werden gevolgd. Hierbij was het van belang te onderzoeken of de semantische en/of syntactische integratie betrokken is bij het maken van een coreferentie tussen een pronoom en zijn antecedent door middel van een schending van de geslachtsinformatie: Bovendien verhoogden wij de afstand tussen het pronoom en het antecedent om te onderzoeken of de syntactische en/of semantische verwerking van pronomen met het verbaal werkgeheugen (verbal working memory, VWM) in wisselwerking zou staan. Door verschillende methodes te gebruiken, konden wij het tijdsverloop (wanneer) van onderzochte processen (event related potentials, ERP) en de lokalisatie (waar) van respectieve neuronaal netwerk (functioneel magnetic resonance imaging, $\mathrm{AMRI}$ ) onderzoeken.

Het literatuur overzicht dat in Hoofdstuk 1 wordt gegeven toont de huidige kemnis over de betrokkenheid van syntactische en semantische informatieverwerking bij het vestigen wan een coreferentie tussen een pronoom en een geschikt antecedent. Tevens werd genoend dat de invloed van extra verwerking toe te schrijven is aan de verhoogde moeilijkheid in zinnen. Alles bij elkaar leidt het overzicht tot drie belangrijke ERP componenten, namelijk de N400 (semantische integratieproblemen), de P600 (her-analyse en moeilijkheidsgraad van syntactische verwerking) en de 'left anterior negativity' (LAN, het werkgeheugen). Neuroimaging onderzoeken ontdekten een complex neuronalal netwerk voor taalbegrip met inbegrip van o.a. inferieure frontale gebieden, superieure en mediale temporale gebieden, en gebieden naast de supramarginale/angulaire gyrus.

In Hoofdstuk 2 tonen wij ERP bevindingen, welke aantoonden dat de schendingen bij zuivere syntactische geslachtsschending (dingen) en bij de combinatie van syntactische en biologische geslachtsschending (personen) op de positie van het pronoom in een P600 worden 
weerspiegeld. Dit effect was groter woor de persoon-conditie dan voor de ding-conditie, wat suggereent dat de P600 gevoelig is voor de beschikbaarheid van semantische geslachtsinformatie van het antecedent (hier, het al dan niet hebben van een biologisch geslacht). Verder wees de wargenomen $\mathrm{N} 400$ voor het woord direct na het pronoom, in het geval van ding maar niet persoon antecedent types, erop dat dit effect continue integratieprocessen voor ding-condities weerspiegelt, terwijl voor persoon-condities de integratie bij de positie van het pronoom lijkt te zijn beěindigd.

In Hoofdstuk 3 wordt gerapporteerd dat, in tegenstelling tot de duidelijke resultaten van het eerste experiment, gelijktijdig geregistreerde MEG (magnetoencephalographic) gegevens, verrassend genoeg geen betrouwbare verschillen lieten zien tussen congruente en incongruente pronomen die naar persoon- of ding-antecedenten terugverwijzen. Dit resultaat wordt verklaard door de verschillende resultaten voor ERP en event related fields (ERF) gegevens, toe te schrijven aan de verschillende kenmerken van de twee methodes (o.a. het meten van divergerende versus radiale bronnen of de ruimtelijke verspreiding van de bronnen voor hogere coginitieve processen). Wanneer de hogere verwerkingsstadia de kern van het onderzoek zijn, zoals bij taalverwerking het geval is, dan kunnen de relevante bronnen ruimtelijk verspreid zijn en kunnen stromen elkaar gedeeltelijk compenseren wanneer die gelijktijdig optreden op tegenover liggende kanten van een sulcus. In dat geval zou kunnen blijken dat ERP de waardevollere bron van informatie is.

Om de onderliggende neuronale netwerken van biologische en syntactische geslachtsintegratie tijdens pronomen verwerking te lokaliseren, voerden wij een event related IMRI experiment uit, dat in Hoofdstuk 4 wordt beschreven. In het algemeen activeert syntactische verwerking gebieden in de linker hemisfeer die naast het gebied van Broca liggen en de superieure temporale sulcus (STs), terwill de verwerking van de biologische geslachtsinformatie tevens een bilateraal netwerk met inbegrip van inferieure frontale gynus (GFi) en supramargihale gyrus (Gsm) omvatte. Een vergelijking met ERPs waarbij identiek materiaal werd gebruikt (experiment 1 in hoofdstuk 2), toonde aan dat de informatie 400-700 $\mathrm{ms}$ na aanvang van de stimulus geïntegreerd wordt, in beide gevallen duidelijk waarneembaar als een P600 met verschillende effect grootte. De resultaten belichten temporele en ruimtelijke integratie van syntactische en semantische verwerking tijdens de totstandbrenging van coreferentic tussen een pronoom en zijn verwijzend zelfstandig naamwoord. Voor pronomen die terugverwijzen naar personen kan het passende antecedent door middel van biologische en 
syntactische informatie gevonden worden. Waarbij meer verwerkingscapaciteit nodig is, wat zichtbaar is in een grote P600 en de activering van een bilateraal corticalal netwerk, met inbegrip van GFi en Gsm/GiA. Het passende antecedent voor pronomen die naar dingen terugverwijzen kan daarentegen alleen door de zuivere syntactische geslachtsinformatie gevonden worden. Dit is zichtbar in een kleine P600 en wijst op minder verwerkingsactiviteit en een activering van linker GFi en STs.

Het integreren van een pronoom in een zinscontext vereist het inzetten van het VWM, omdat het eerder vermelde antecedent moet worden gereactiveerd on het pronoom met het antecedent te verbinden. In het experiment beschreven in Hoofdstuk 5 manipuleerden wij dit proces door de afstand bij persoon-zinnen en ding-zimnen te verhogen (long distance conditions, LD) en we vergeleken deze met de zimnen van de voorgaande experimenten (short distance conditions, SD) bimnen één ERP experiment. In het algemeen beïnvloedde deze manipulatie van het VWM, de N400/P600 patronen van onze zinstypes waaruit blijkt dat het VWM betrokken is bij semantische en syntactische verwerking. Tevens wijzen onze gegevens er op dat de zinsontleding niet alleen van semantische of syntactische informatie afhangt, maar dat het eerder een hoogst flexibel proces is. De eerder vermelde N400/P600 patronen wijzen er op dat het begripssysteem tijdens de verwerking van pronomen, schakelt tussen voorkeur voor semantische en syntactische informatie, afhankelijk van belasting van het VWM en beschikbaarheid van informatie. Bovendien vonden wij een N400 op het woord na het pronoom yoor SD en LD ding-zinnen, wat wijst op een integratieproces dat doorgaat na de positie van het pronoom, onafhankelijk van de afstandsmanipulatie.

Het doel van de fMRI studie die in Hoofdstuk 6 wordt vermeld, was het lokaliseren van de verwerking van syntactische en semantische geslachtsinformatie en de mogelijke interactie met het VWM tijdens het proces dat coreferentie tussen een pronoom en zijn antecedent opbouwt. Bij dat onderzoek werd gebruik gemaakt van het materiaal dat in hoofdstuk vijf wordt beschreven. Als hoofdresultaat vonden wij dat de linker inferieure frontale gebieden het kerngebied zijn voor het opbouwen van een coreferentie tussen pronoom en antecedent met een lichte lokale differentiatie afhankelijk van antecedent type. Bovendien stellen wij dat de linker superieure frontale gebieden beschouwd kunnen worden als een algemene controlerende verwerkingseenheid voor duidelijke geslachtsschendingen binnen relatief gemakkelijke SD zimnen, aangezien wij bimen deze gebieden een deactivering voor incongruente pronomen vonden in vergelijking met congruente pronomen, maar dit njet 
vonden voor de LD zinnen. In plaats daarvan zagen wij extra activering binnen temporale en temporoparietale gebieden, als het begripssysteem zich slechts op syntactische geslachtsinformatie kan baseren om het pronoom met een ver antecedent (thet LD ding) te verbinden. Het zoeken naar alternatieve antecedenten werd geïndiceerd door een activering van hersenengebieden die gewoonlijk aan semantische informatieverwerking van verwijzingea worden toegeschreven, namelijk Gsm. Bovendien toonden wij aan dat de gevoeligheid van de afstandsmanipulatie afhankelijk is van de beschikbaarheid van geslachtsinformatie bimnen overeenstemmende zinnen, wat in een activering van temporele gebieden voor $L D$ in vergelijking met de zinnen van de persoon wan SD werd weerspiegeld, terwijl in het geval wan een ding geen activering van temporele gebieden werd gevonden. Voor incongruente zimnen vonden wij voorafgaand aan de activering van temporele gebieden, voor de antecedenten van de persoon en een meer latere activering van temporele gebieden voor de antecedenten van het ding. Wij stellen dat deze gebieden geactiveerd worden vanwege het zoeken naar het passende antecedent, afhankelijk van informatiebeschikbaarheid in het geval van schendingen, maar ook in het geval van een langere temporele afstand (LD) tussen antecedenten en pronomen. 


\section{References}

Baayen, R.H., Piepenbrock, R., \& Gulikers, L. (1995). The CELEX lexical database (CDROM). Philadelphia: Linguistic Data Consortium, University of Pennsylvania.

Baddeley, A. (1998). Recent development in working memory. Current Opinion in Neurobiology. 8, 234-238.

Brown, C.M., Hagoort, P., \& Kutas, M. (2000). Postlexical integration processes in language comprehension: Evidence from brain imaging research. In: Gazzaniga, M. S. (Ed.) The New Cognitive Neuroscience. MIT Press, Cambridge, pp. 881-895.

Buckner, R.L., Bandettini, P.A., O'Craven, K.M., Savoy, R.L., Petersen, S.E., Raichle, M.E., \& Rosen, B.R. (1996). Detection of cortical activation during averaged single trials of a cognitive task using functional magnetic resonance imaging. Proceedings of the National Acadamy of Science, 93, 14878-1.4883.

Carpenter, P.A., Miyake, A., \& Just, M.A. (1995). Language comprehension: sentence and discourse processing. Annu. Rev Psychol. 46, 91-120.

Carreiras, M., Garnham, A., Oakhill, J., \& Cain, K (1996). The use of stereotypical gender information in constructing a mental model: evidence from English and Spanish. Quarterly Journal of Experimental Psychology Section A-Human Experimental Psychology, 34, 41-62.

Cooke, A., Zurif, E. B., DeVita, C., Alsop, D., Koenig, P.., Detre, J., Gee, J., Pinango, M., Balogh, J., \& Grossman, M. (2001). Neural basis for sentence comprehension: Grammatical and short-term memory components. Human Brain Mapping, 15, 80-91.

Dale, A.M., \& Buckner, R.L. (1997). Selective averaging of rapidly presented indiwidual trials using fMRI. Human Brain Mapping. 5, 329-340.

Dale, A.M., \& Sereno, M.I. (1993). Improved Localization of Cortical Activity by combining EEG and MEG with cortical Surface Reconstruction: A linear approach. Journal of Cognitive Neuroscience, 5, 162-176.

Damasio, H., Gabrowski, T.J., Tranle, D., Hichwa, R.D., \& Damasio, A.R. (1996). A neural basis for lexical retrieval. Nature, 380, 499-505.

Daneman, M., \& Carpenter, P.A. (1980). Individual differences in working memory and reading. Journal of Verbal Learning and Verbal Behaviour. 19,450-466. 
Dell, G.S., McKoon, G, \& Rateliff, R. (1983). The activation of antecedent information during the processing of anaphoric reference in reading. Joumal of verbal Learning Behavior, 22, 121-132.

Eulitz, C., Eulitz, H, \& Elbert, T. (1997). Differential outcomes from magneto- and electroencephalography for the anallysis of human cognition. Neuroscience Letter, 227, 185-188.

Ferreira, F., \& Clifton, C.Jr. (1986). The independence of syntactic processing. Joumal of Memory and Language, $25,348-368$.

Fiebach, C.J., Schlesewsky, M., \& Friederici, A.D. (2001). Syntactic working memory and the establishment of filler-gap dependencies: insights from ERPs and MMRI. Journal of Psycholinguistic Research, 30, 321-338.

Fischl, B., Sereno, M. I., Tootell, R.B., \& Dale, A.M. (1999). High-resolution intersubject averaging and a coordinate system for the cortical surface. Human Brain Mapping. 8, 272-284.

Fischl, B., Sereno, M.I., \& Dale, A.M. (1999). Cortical surface-based analysis: II. Inflation, flattening, and a surface-based coordinate system. Neuroimage. 9, 195-207.

Firauenfelder, U.H., \& Tyler, L.K. (1987). The process of spoken word recognition: An introduction. Cognition, 25, 1-20.

Frazier, L., \& Fodor, J.A. (1987). The sausage machine: A new two-stage parsing model. Cognition, 6, 291-325.

Friederici, A.D. (2002). Towards a neural basis of auditory sentence processing. Trends Cognitive Science, $6,78-84$.

Friederici, A.D., \& Mecklinger, A. (1996). Syntactic parsing as revealed by brain responses:

First pass and second pass parsing process. Journal of Psycholinguistic Research, 25, $157-176$.

Friederici, A.D., Hahne, A., \& Mecklinger, A. (1996). Temporal structure of syntactic parsing: early and late event-related brain potential effects. Joumal of Experimental Psychology: Learning, Memory, and Cognition, 22, 1219-1248.

Friederici, A.D., Pfeifer, E., \& Hahne, A. (1993). Event-related brain potentials during natural speech processing: effects of semantic, morphological and syntactic violations. Brain Res Cogn Brain Res. 1, 183-192.

Friederici, A.D., Ruschemeyer, S. A., Hahne, A., \& Fiebach, C.J. (2003). The role of left inferior frontal and superior temporal cortex in sentence comprehension: localizing syntactic and semantic processes. Cerebral Cortex, 13, 170-177. 
Friederici, A.D., Steimhauer, K., \& Frisch, S. (1999). Lexical integration: sequential effects of syntactic and semantic information. Memory, \& Cognition, 27, 438-453.

Friederici, A.D., Steinhauer, K., Mecklinger, A., \& Meyer, M. (1998). Working memory constraints on syntactic ambiguity resolution as revealed by electrical brain responses. Biological Psychology, 47, 193-221.

Friederici, A.D., Wang, Y., Hermann, C.S., Maess, B., \& Oertel, U. (2000). Localization of early syntactic processes in frontal and temporal cortical areas: a magnetoencephalographic study. Human Brain Mapping, 11, 1-11.

Frisch, S., \& Schlesewsky, M. (2001). The N400 reflects problems of thematic hierarchizing. Neuroreport, 12,3391-3394.

Gabrieli, J.D.E., Poldrack, R.A., \& Desmond, J.E. (1998). The role of left prefrontal cortex in language and memory. Proc. Nat. Acad. Sci. USA. 95, 906-913.

Garnham, A. Oakhill, F., Ehrlich, M.F., \& Carreiras, M. (1995). Representations and processes in the interpretation of pronouns: new evidence from Spanish and French. Journal of Memory, \& Language, 34, 41-62.

Garrod, S.C., \& Sanford, A.J. (1994). Resolving sentences in a discourse context. In: Gernsbacher, M. A. (Ed.), Handbook of Psycholinguistics. Academic Press, New York, pp. 675-698.

Garrod, S.C., \& Terras, M. (2000). The contribution of lexical and situational knowledge to resolving discourse roles: bonding and resolution. Journal of Memory, \& Language. 42, 526-544.

Garrod, S.C., Freudenthal, D., \& Boyle, E. (1994). The role of different types of anaphor in the on-line resolution of sentences in a discourse. Journal of Memory, \& Language, 33 , 39-68.

Genovese, C.R., Lazar, N.A., \& Nichols, T. (2002). Thresholding of statistical maps in functional Neuroimaging using Fallse Discovery Rate. Neuroimage, 15, 870 - 878.

Gernsbacher, M.A. (1991). Comprehending conceptual anaphors. Language and Cognitive Processes, 6, 81-105.

Gordon, P.C., \& Hendrick, R. (1998). The representation and processing of coreference in discourse. Cognitive Science, 22, 389-424.

Graesser, A.C., Millis, K.K., \& Zwaan, R.A. (1997). Discourse comprehension. Annual Reviews in Psychology, 48, 163-189.

Gunter, T.C., Stowe, L.A., \& Mulder, G. (1997). When syntax meets semantics. Psychophysiology. 34, 660-676. 
Hagoort, P. (2003). How the brain solves the binding problem for language: a neurocomputational model of syntactic processing. Neuroimage, $20,18-29$.

Hagoont, P., Brown, C.M. \& Groothusen, J. (1993). The syntactic positive shift (SPS) as an ERP measure of syntactic processing. Language and Cognitive Processes, 8, $439-483$.

Hagoort, P., Brown, C.M.* \& Osterhout, L. (1999). The neuroeognition of syntactic processing. In: Brown, C., \& Hagoort, P. (Eds.), The neurocognition of language, University Press, Oxford, pp. 273-316.

Hagoort, P., Hald, L., Bastiaansen, M., \& Petersson, K.M. (2004). Integration of Word Meaning and World Knowledge in Language Comprehension. Science 304, 438-441.

Hahne, A.* \& Eriederici, A. D. (1999). Electrophysiological evidence for two steps in syntactic analysis. Early automatic and late controlled processes. Journal of Cognitive Neuroscience, 11, 194-205.

Halgren, E., Dhond, R.P., Christensen, N., Van Petten, C., Marinkovic, K., Lewine, J.D., \& Dale, A.M. (2002). N400-like magnetoencephalography responses modulated by semantic context, word frequency, and lexical class in sentences. Neuroimage, 17, 11011116.

Hämăläinen, M., Hari, R., Ilmoniemi, R.J., Knuutila, J., \& Loumasmaa, O.V. (1993). Magnetoencephalography - theory, instrumentation and applications to non-invasive studies of the working human brain. Rev Mod Phys, 65, 413-497.

Hammer, A., Jansma, B.M., Lamers, M., \& Mïnte, T.F. (2005). Pronominal Reference in Sentences about Person or Things: An electrophysiological approach. Journal of Cognitive Neuroscience. 17, 227-239.

Hankamer, J., \& Sag, I.A. (1976). Deep and surface anaphora. Linguistic Inquiry, 7, 391-428.

lndefrey, P., Hagoort, P., Herzog, H., Seitz, R. J., \& Brown, C.M. (2001). Syntactic processing in left prefrontal cortex is independent of lexical meaning. Neuroimage, 14, 546-555.

Kaan, E., Harris, A., Gibson, E., \& Holcomb, P. (2000). The P600 as an index of syntactic integration difficulty. Language and Cognitive Processes. 15, 159-201.

Kiehl, K. A., Laurens, K. R., \& Liddle, P.F. (2002). Reading anomalous sentences: an eventrelated $\mathrm{fMRI}$ study of semantic processing. Neuroimage, 17, 842-850.

King, J.W., \& Kutas, M. (1995). Who did what and when? Using word- and clause-level ERPs to monitor working memory usage in reading. Journal of Cognitive Neuroscience, $7,376-395$. 
King, S.W., \& Kutas, M. (1997). Is she an engineer? Brain potentials and anaphora Poster presentation at the Fourth Annual Meeting of the Cognitive Neuroscience Society (Boston).

Kluender, R., \& Kutas, M. (1993). Bridging the gap: Evidence from ERPs on the processing of unbounded dependencies. Journal Cognitive Neuroscience, 5, 196-214.

Kolk, H.H.J., Chwilla, D.J., van Herten, M., \& Oor, P.J.W. (2003). Structure and limited capacity in verbal working memory: A study with event-related potentials. Brain and Language, $85,1-36$.

Kriegeskorte, N., \& Goebel, R. (2001). An efficient algorithm for topologically correct segmentation of the cortical sheet in anatomical mr volumes. Neuroimage, 14, 329-346.

Kuperberg, G.R., Holcomb, P.J., Sitnikova, T., Greve, D., Dale, A.M., \& Caplan, D. (2003). Distinct patterns of neural modulation during the processing of conceptual and syntactic anomalies. Journal of Cognitive Neuroscience, 15, 272-293.

Kutas, M., \& Hillyard, S.A. (1980). Reading between the lines"." Event-related brain potentials during natural sentence processing. Brain, \& Language, 11, 354-373.

Kutas, M., \& Hillyard, S.A. (1983). Event-related brain potentials to grammatical errors and semantic anomalies. Memory, \& Cognition, 11, 539-550.

Kutas, M., \& King, J.W. (1995). The potentials for basic sentence processing: differentiating integrative processes. In: Inui, T., \& McClelland, J. L. (Eds.), Information Integration in Perception and Communication, Attention and Performance, Vol. 16, MIT Press, Cambridge, MA, 1996, pp. $501-546$.

Kutas, M., \& Schmitt, B.M. (2003) Language in Microvolts. $\mathbb{1 n}$ : Banich, M.T., \& Mack, M. A. (Eds.), Mind, Brain, and Language: Multidisciplinary Perspectives. Erlbaum Assoc. Inc., Lawrence, pp. 171-209.

Kutas, M., \& van Petten, C.K. (1994). Psycholinguistics electrified: event-related brain potential investigations. In: Gernsbacher, M. A. (Ed.), Handbook of Psycholinguistics. Academic Press, San Diego, pp. 83-143.

Kutas, M., Federmeier, K.D., Coulson, S., King, J.W., \& Münte, T.F. (2000). Language. In: Cacioppo, J. T., Tassinary, G., \& Berntson, G. G. (Eds.), Handbook of Psychophysiology, 2nd Edition, Cambridge University Press, pp. 576-601.

Lütkenhöner, B., Menninghaus, E., Steinsträter, O,, Wienbruch, C., Gißler, M., \& EIber, T. (1995). Neuromagnetic source analysis using magnetic resonance images for the construction of source and volume conductor model. Brain Topography, 7, 291-299. 
Lutzenberger, W., Elbert, T., \& Roekstroh, B. (1987). A brief tutorial on the implications of volume conduction fort he interpretation of the EEG, Journal of Psychophysiology, 1 , 81-89.

MacDonald, M.C., \& MacWhmey, B. (1990). Measuring inhibition and facilitation from pronouns, Journal of Memory, \& Language, 29, 469-492.

Marslen-Wilson, W.D., \& Tyler, L.K. (1980). The temporal structure of spoken language understanding. Cognition, 8, 1-71.

Martin, A., \& Chao, L.L. (2001). Semantic memory and the brain: structure and processes. Current Opinion in Neurobiology, 11, 194-201.

Münte, T.F., Heinze, H. J., Matzke, M. Wieringa, B.M., \& Johannes, S. (1998a). Brain potentials and syntactic violations revisited: no evidence for specificity of the syntactic positive shift. Neuropsychologia, 36, 217-226.

Münte, T.F., Heinze, H.-J., \& Mangun, G.R. (1993). Dissociation of brain activity related to syntactic and semantic aspects of language. Journal of Cognitive Neuroscience, 5, 335344.

Münte, T.F., Schiltz, K., \& Kutas, M. (1998b). When temporal terms belie conceptual order. Nature, 395, 71-73.

Münte, T.F., Urbach, T.P., Düel, E., \& Kutas, M. (2000). Event-related brain potentials in. the study of human cognition and neuropsychology. In: Boller, F., Grafman, J., \& Rizzolatti (Eds.), Handbook of Neuropsychology, Vol. 1, Elsevier, Amsterdam.

Münte, T.F., Wieringa, B.M., Weyerts, H., Szentkuti, A., Matzke, M., \& Johannes, S. (2001). Diflerences in brain potentials to open and closed class words: class and frequency effects. Neuropsychologia, 39, 91-102.

Neville, H., Nicol., J. L., Barss, A., Forster, K. I., \& Garret, M. F. (1991). Syntactically based sentence processing classes: Evidence from event-related brain potentials. Journal of Cognitive Neuroscience, $3,151-165$.

Newman, A.J., Pancheva, R., Ozawa, K., Neville, H. I., \& Ullman, M.T. (2001). An eventrelated PMRI study of syntactic and semantic violations. Journal of Psycholinguist Research, 30, 339-364.

Nicol, J., \& Swimney, D. (1989). The role of structure in coreference assignment during sentence comprehension. Journal of Psycholinguist Research, 18, 5-19.

Osterhout, L., \& Holcomb, P.J. (1992). Event-related brain potentials elicited by syntactic anomaly. Journal of Memory, \& Language, 34, 785-806. 
Osterhout, L., \& Mobley, L.A. (1995). Event-related brain potentials elicited by failure to agree. Journal of Memory, \& Language, 34, 739-773.

Osterhout, L., \& Nicol, J. (1999). On the Distinctiveness, Independence, and Time Course of the Brain Responses to Syntactic and Semantic Anomalies. Language and Cognitive Processes, 14, 283-317.

Osterhout, L., Bersick, M., \& McLaughlin, J. (1997). Brain potentials reflect violations of gender stereotypes. Memory, \& Cognition, 25, 273-285.

Osterhout, L., Holcomb, P.J., \& Swinney, D.A. (1994). Brain potentials elicited by gardenpath sentences: evidence of the application of verb information during parsing. Journal of Experimental Psychology: Learning, Memory, and Cognition, 20, 786-803.

Papanicolaou, A.C. (1998). Fundamental of functional brain imaging. A guide to the methods and their applications to psychology and behavioral neurosciences. Swets, \& Zeitlinger B.V., Lisse. The Netherlands.

Rigalleau, F., \& Caplan, D. (2000). Effects of gender marking in pronominal coindexation. The Quarterly Journal of Experimental Psychology, 53, 23-52.

Röder, B., Stock, O., Neville, H., Bien, S., \& Rösler, F. (2002). Brain activation modulated by the comprehension of normal and pseudo-words of different processing demands: $A$ functional Magnetic Resonance Imaging study. Neuroimage, 15, 1003-1014.

Rösler, F., Pechman, T., Streb, J., Röder, B., \& Hennighausen, E. (1998). Parsing of sentences in a language with varying word order: word-by-word variations of processing demands are revealed by event-related brain potentials. Journal of Memory and Language, 38 , 150-176.

Rugg, M.D., \& Coles, M.G.H. (1995). The ERP and cognitive psychology: conceptual issues. In: Rugg, M. D., \& Coles, M. G. H. (Eds.), Electrophysiology of Mind: Event-related Brain Potentials and Cognition. Oxford University Press, New York, pp. 27-39.

Schmitt, B.M., Lamers, M., \& Münte, T.F. (2002). Electrophysiological estimates of biological and syntactic gender violation during pronoun processing. Cognitive Brain Research, 14, 333-346.

Simos, P.G., Basile, L.F., \& Papanicolaou, A.C. (1997). Source localization of the N400 response in a sentence-reading paradigm using evoked magnetic fields and magnetic resonance imaging. Brain Research, 762, 29-39.

St. George, M., Kutas, M., Martinez, A., \& Sereno, M.l. (1999). Semantic integration in reading: engagement of the right hemisphere during discourse processing. Brain, 122 , 1317-1325. 
Stefanovic, B., Warnking, J.M., \& Pike, G.B. (2004) Hemodynamic and metabolic responses to neuronal inhibition. Neuroimage, 22, 771-778.

Streb, J., Rösler, F., Hennighausen, E. (1999): Event-related responses to pronoun and proper anaphors in parallel and nomparallel discourse structures. Brain and Language, $70,273-286$.

Swab, T.Y., Camblin, C.C., \& Gordon, P.C. (2004). Electrophysiological evidence for reversed lexical repetition effects in language processing. Journal of Cognitive Neuroscience, $16,1-12$.

Talairach, J., \& Tournoux, P. (1988). Co-planar stereotaxíc atlas of the human brain, Ist ed. Thieme Medical Publishing Company, New York.

Thierry, G., Ibarrola, D., Demonet, J.F.\& Cardebat; D. (2003). Demand on verbal working memory delays haemodynamic response in the inferior prefrontal cortex. Hum Brain Mapping, 19, 37-46.

Tichonov, A.N., \& Arsenin, V.Y. (1977). Solution of Ill-posed Problems. Washington D.C. Winston (English translation by F.John).

Trueswell, J.C., \& Tanenhaus, M.K. (1994). Toward a lexicalist framework for constraintbased syntactic ambiguity resolution. In C. Clifton, Rayner, K., \& Frazier, L. (Eds.), Perspectives on Sentence Processing. Hillsdale, NJ: Lawrence Erlbaum Associates.

Van Berkum, J.J., Brown, C.M., Hagoort, P., \& Zwitserlood, P. (2003). Event-related brain potentials reflect discourse-referential ambiguity in spoken language comprehension. Psychophysiology, 40, 235-248.

Van Berkum, J.J., Hagoort, P., \& Brown, C.M. (1999). Semantic integration in sentences and discourse: Evidence from the N400. Journal of Cognitive Neuroscience, 11, 657-671.

Van Petten, C., \& Kutas, M. (1991). Influences of semantic and syntactic context on openand closed-class words. Memory, \& Cognition, 19, 95-112.

Vosse, T., \& Kempen, G.A.M. (2000). Syntactic structure assembly in human parsing: a computational model based on competitive inhibition and lexicalist grammar. Cognition. $75,105-1.43$.

Wagner, A.D. (1999). Working memory contributions to human learning and remembering. Neuron, 22, 19-22.

Williamson, J.S., \& Kaufinan, L. (1990). Theory of neuromagnetic fields. In F. Grandori, M. Hoke, \& G.L. Romani (Eds.). Auditory Evoked Magnetic Fields and Electrical Potential, Vol. 6, Advances in Audiology, Karger, Basel, 1990, pp. 1-39. 


\section{Appendix I}

Materials for Chapter 2 (experiment 1), Chapter 3, and 4

\section{A: Person as antecedent:}

1. Die Frau ist beliebt, weil sie/er hübsch ist.

2. Die Nonne ist fromm, weil sie/er glatubig ist.

3. Die Tante ist akkurat, weil sie/er Ordnung mag.

4. Die Oma liest gern, weil sie/er Geschichten mag.

5. Die Prinzessin war munter, weil sie/er Schlaf bekam.

6. Die Tochter ist krank, weil sieler angesteckt wurde.

7. Die Nichte ist allein, weil sie/er oft umzog.

8. Die Schülerin hat geschwänzt, weil sie/er faul war.

9. Die Dame ist traurig, weil sie/er einsan ist.

10. Die Hexe ist aufgeregt, weil sie/er Besuch hat.

11. Die Witwe nuss weinen, weil sie/er traurig ist.

12. Die Beamtin ist gestresst, weil sie/er Kinder hat.

13. Die Studentin ist nervös, weil sie/er Prïfungen hat.

14. Die Baronin ist glucklich, weil sie/er bald heiratet.

15. Die Ballerina schreibt Autogramme, weil sie/er beruhmt ist.

16. Die Freundin backt Kuchen, weil siejer Geburtstag hat.

17. Die Sekretärin ist verärgert, weil sie/er Überstunden macht.

18. Die Königin ist beliebt, weil sie/er gut ist.

19. Die Arztin ist klug, weil sie/er gelernt hat.

20. Die Braut ist zittrig, weil sie/er aufgeregt ist.

21. Die Chefin ist verzweifelt, weil sie/er betrogen wurde.

22. Die Diva ist stur, weil sie/er hochnäsig ist.

23. Die Dozentin ist streng, weil sieler viel fordert.

24. Die Enkelin ist niedlich, weil sie/er klein ist.

25. Die Spielerin ist arm, weil sie/er viel verliert.

26. Die Patientin ist besorgt, weil sie/er krank ist.

27. Die Săngerin ist fröhlich, weil sie/kr viel singt.

28. Die Schneiderin ist froh, weil sie/er Stoff kaufte.

29. Die Schwester ist witend, weil sie/er geärgert wird.

30. Die Stewardess ist arbeitslos, weil sie/er llugangst bekam.

31. Die Zigeunerin ist beweglich, weil sie/er viel tanzt.

32. Die Zeugin ist tungstlich, weil sie/er bedroht wird.

33. Die Erzieherin ist müde, weil sie/er viel arbeitet.

34. Die Gattin ist sparsam, weil sie/er arm ist.

35. Die Jounalistin ist verreist, weil siejer Entspannung sucht.

36. Die Professorin ist anwesend, weil sie/er Besprechungen fhat.

37. Die Rentnerin ist wach, weil sieler Kaffee trank.

38. Die Sklavin ist hungrig, weil sieter wenig isst.

39. Die Schwedin ist musikalisch, weil sie/er Klavier spielt.

40. Die Konkurrentin ist schroff, weil sieter benachteiligt wurde,

41. Die Pflegerin ist geduldig, weil sie/er warten kann.

42. Die Forscherin ist wissbegierig, weil sief er interessiert ist.

43. Die Kundin ist modisch, weil sie/er gefallen möchte.

44. Die Lehrerin unterrichtet gut, weil sie/ers spontan ist.

45. Die Kandidatin ist betrubt, weil sie/ar schwanger wurde.

46. Die Kameradin war böse, weil sieler zuhause blieb.

47. Die Jungfer wird ignoriert, weill siejer klein isi. 
48. Die inist spontan, weil steror verre

49. Die Kauffraw ist werychuld, weil siefer unaufmerksam war.

50. Die kelneritist misicher, well gieler herte beginnt.

51. Die Kochin ist werschwitz, weil sider wiel arbeitet.

52. Die Greisin ist weise, weil sie/er alt ist.

53. Die Heldin erlebt Aberteuer, weil sie/er mutig ist.

54. Dix Magd hat frei, weil sie/er Urlaub hat.

55. Die Plondine wolle schwimmen, weil siefer Wasser mag.

56. Die Kaiserin ist ideenreich, well sie/er kreativ ist.

57. Die Arbeiterin ist eingestellt, weil sie/er zuverlássig ist.

58. Die Polin ist werlobt, well siefer verliebt ist.

59. Die Gouveriante kauft Kleider, weil side fer eitel ist.

60. Die Gottin wird verehrt, well sieter großzügig ist.

61. Dè Englünder ist errồtet, weil er/sie schüchtern ist.

62. Der Abiturient ist schnell, weil er/sie lwufen kann.

63. Der Polizist wirkt autoritat, weil er/sie Uniformen tuägt.

64. Der Arzt ist diberarbeitet, weil er/sie Patienten betreut.

65. Der Herr ist reich, weil erfsie geizig ist.

66. Dert Schauspieler ist erfolgreich, weill er/sie ehrgeizig ist.

67. Der Schlosser ist schmutzig, weil er/sir viel arbeitet.

68. Det Vater ist ungeduldig, well er/sie Ruhe braucht.

69. Der Ingenieur ist unerfahren, weil er/sie jung ist.

70. Det Faluer ist gefahrich, weil er/sre getrunken hat.

71. Der Sanger ist bekannt, weil er/sie fantastisch singt.

72. Der Patient hustet viel, weil er/sie zuviel raucht.

73. Der Dichter ist poetisch, weil er/sie Gedichte schreibt.

74. Der Pianist ist einsam, weil er/sie viel teist.

75. Der Spieler ist enttascht, weil er/sie verloren hat.

76. Der Bengel ist langsam, weil er/sie verletzt ist.

77. Der Kơnig ist mächtig, weil er/sie reich ist.

78. Der Bruder ist sportlich, weil er/sie viel trainient.

79. Der Soldat ist kuâftig, weil er/she Fitness betreibt.

80. Der Anwalt schlant schlecht, weil er/sie beuruhigt ist.

81. Der Pirat ist betrunken, weil er/sie heute feiert.

82. Der Rentner ist drawBen, weil er/sie gern wandert.

83. Der Richter ist werreist, weil er/ste Termine hat.

84. Der Ritter ist verletat, weil er/sie gekdmpft hat.

85. Dor Profesisor ist beschaftigt, weil er/she Arbeit hat.

86. Der Fischer ist beruthigt, weil ew/sie angeln kann.

87. Der Geselle ist unachtsam, weil er/sie mude ist.

88. Der Chef war spat, weil er/sie verschlafen hat.

89. Der Baron ist dick, weil er/sie viel isst.

90. Der Clown ist bunt, weil en/she warkleidet ist.

91. Der Diener ist anerkannt, weil ar/sie fleibig ist.

92. Der Chirurg ist enst, weil er/sio gerade operient.

93. Der Laborant ist shohchtern, weil ev/sie frend ist.

94. Der Freund ast neugierig, weil er/sie Gerilichte hörte.

95. Der Hafting ist phffig, weil erisie fluchten konnte.

96. Der Held ist stolz, weil er/sie gefeiert wird.

97. Der Gatte ist schlafrig, weil er/sie nachts wacht.

98. Der Mann ist geschickt, weil ar/sie viel übt.

99. Den Knecht ist dionn, weil erisie kaum isst.

100. Det Narr ist wertuckt, weil er/sit Unsinn treibt.

101. Den Hauptling ist neidisch, weil en/sie keine frau bekonmt.

102. Der Neffe schleckt Eis, well er/sie Subes mag.

103. Der Jager ist ordentlich, well er/sie stuchen hasst.

104. Del Opa ist zerstreut, weil er/sie alt ist.

105. Det Onkel kautt Sül3es, weil er/sie Geschenke brancht.

106. Der Spion ist gespannt, weil er/sie Auftrage hat. 
107. Der Schüler ist fleißig, weil er/sie Klausuren schreibt.

108. Der Kapitän kennt Länder, weil er/sie viel segelt.

109. Der Koch ist dickköpfig, weil er/sie egoistisch ist.

110. Der Pfarrer trägt schwarz, weil er/sie Gottesdienst halt.

111. Der Prinz ist eitel, weil er/sye schön ist.

112. Der Kommissar ist effizient, weil er/sie arbeiten muss.

113. Der Monteur ist tatkräftig, weil er/sie bald verreist.

114. Der Zauberer ist witzig, weil er/sie Humor hat.

115. Der General ist interessiert, weil er/sie Projekte hat.

116. Der Hirte ist zuverlässig, weil er/sie Schafe hätet.

117. Der Kamerad ist bescheiden, weil er/sie Freunde hat.

118. Der Journalist ist grofizügig, weil er/sie Fotos hat.

119. Der Kaiser ist schlau, weil er/sie Plane hat.

120. Der Greis ist verschnupft, weil er/sie erkältet ist.

\section{B: Thing as antecedent:}

1. Die Tur ist offen, weil sieler kaputt ist.

2. Die Glocke ist laut, weil sie/er groß ist.

3. Die Vase ist kaputt, weil sie/er gefallen ist.

4. Die Bluse ist schön, weil sie/er gelb ist.

5. Die Aufgabe ist schwer, weill sie/er unlösbar ist.

6. Die Flasche ist ausgelaufen, weil sie/er undicht war.

7. Die Blume ist verblüht, weil sie/er vertrocknet ist.

8. Die Hose ist schmutzig, weil sie/er sclulammig ist.

9. Die Tasche ist praktisch, weil sie/er iubersichtlich ist.

10. Die Maschine ist laut, weil sie/er wackelig steht.

11. Die Schüssel wird weggeworfen, weil sie/er Risse hat.

12. Die Frisur ist furchtbar, weil sie/er misslungen ist.

13. Die Kneipe ist voll, weil sie/er beliebt ist.

14. Die Jacke ist warm, weil sie/er gefütteit ist.

15. Die Uhr ist genau, weil sie/er gestellt wurde.

16. Die Suppe ist scharf, weil sieler gewarzt wurde.

17. Die Ampel ist aus, weil sie/er defekt ist.

18. Die Heizung ist heiß, weil sie/er an ist.

19. Die Fabrik ist zerstört, weil sie/er unmodern ist.

20. Die Idee ist genial, weil sie/er einfach ist.

21. Die Socke riecht schlecht, weil sie/er ungewaschen ist.

22. Die Stange ist schwer, weil sieler bleien ist.

23. Die Nase trieft stark, weil sie/er verschnupft ist.

24. Die Galerie ist interessant, weil sieler Werke zeigt.

25. Die Salbe ist lindernd, weil sie/er Schmerzen stillt.

26. Die Limonade ist kalt, weil sie/er gekilhlt wurde.

27. Die Truhe ist braun, weil sie/er holzern ist.

28. Die Wunde ist sclumerzend, weil sie/er tief ist.

29. Die Stufe ist schwierig, weil sie/er hoch ist.

30. Die Schere ist scharf, weill sie/er geschliffen wurde.

31. Die Sonne blendet stark, weil sie/er grell ist.

32. Die Schule ist geschlossen, weil sie/er renoviert wird

33. Die Perle ist wertwoll, weil sider selten ist.

34. Die Decke ist unerreichbar, weil sie/er hoch ist

35. Die Scheibe ist klar, weil sie/er gepulzt wurde.

36. Die Butter ist zerlaufen, weil sie/er erwarmt wurde.

37. Die Banane ist matschig, weil sie/er iberreif ist.

38. Die Tablette ist ungesund, weil sie/er giftig ist.

39. Die Frucht ist gesund, weil sie/er Vitamine enthalt.

40. Die Schiene wird gewechselt, weil sie/er porös ist.

41. Die Gabel hat Gewicht, weil sie/er metallen ist.

42. Die Couch ist bequem, weil sie/er gepolstert ist.

43. Die Tasse ist leer, weil sie/er ausgetrunken wurde. 


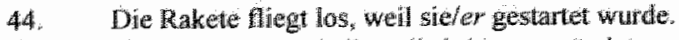

45. Die Kerze brant hell, weil sie/er angezundet wurde.

46. Die Oitare ist harmonisch, weil sie/er gestimmt wurde.

47. Die Kette ist teuer, weil sie/er antik ist:

48. Die Halle ist riesig, weil siefer vergoffert whurde.

49. Die Schachtel hist deformier, well sie/er zendrtekt wute.

50. Die Karte ist gezinkt, well sie/er markiert whirde.

51. Die Tufiel ist beschrieben, weil sie/er genutzt wurde:

52. Die Brille ist durchichitig, weil sieler glasen ust.

53. Dia Kunke ist ha, weil side/ lackert wurde.

54. Die Straße ist glat; weil sie/er vereist ist.

55. Die Kirche ist kalt, weil sle/er ungeheizt ist.

56. Die Sohle ist 18chrig, weil sie/er durchgelaufen ist.

57. Die Krone farkelt hell, weil sie/er golden ist.

58. Die Binde ist rôllich, weil sie/er blutig ist:

59. Die "Tinte ist verschmient, weil sieler fieucht war.

60. Die Krawate ist schick, weil sie/er modera ist.

61. Der Tunnel ist dunkel, weil er/sie lang ist.

62. Der Wagel ist rostig, weil er/suie nass war.

63. Der Fernseher ist gut, weil er/sie neu ist.

64. Der Zahn ist hăsslich, weil er/sre gelb ist.

65. Der Tee ist schledh, well er/sie biltter ist.

66. Der Schuh ist kaput, weil er/sie alt ist.

67. Der Fuller "ist pink, weil er/sie auffallen soll.

68. Der Zug ist spatt, weil er/sie angehalten wurde.

69. Der Roman ist vergriffen, weil ed/sie aufregend ist.

70. Der Mund ist rot, weil er/sie durchblutet ist.

71. Der Kaffee ist stark, weil er/sie aufwecken soll.

72. Der Zettel ist verschwunden, weil en/sie winzig war.

73. Der Garten ist verwildert, weil er/sie unbewohnt ist.

74. Der Topf ist unbranchbar, weil er/sie lochrig hat.

75. Der Rucksack ist schwer, weil er/sie gepackt ist.

76. Der Anzug ist unbeguem, weil er/sie steif ist.

77. Der Braten ist schmackhaft, weil er/sie gar ist.

78. Der Apfel ist suB, weil er/sie reif ist.

79. Der film ist spannend, weil er/sie neu ist.

80. Der Wein schneckt gut, weil er/sie trocken ist.

81. Der Herd ist hein, weil er/sie angestell ist.

82. Der Zaun ist hoch, weil ertsie schoizen soll.

83. Der Gesang ist melodisch, weil er/she harmonisch ist.

8. Der. Salt ist schmackhaft, weil er/sie frisch ist.

85. Der Sessel ist schwer, weil ar/sie grof ist.

86. Der Tag ist mild, weil er/sie sonnigg ist.

87. Der Wecker ist gestellt, weil er/ste klingeln soll.

88. Der Bach ist geschitzt, weil er/she rein ist.

89. Der Hals ist geschwollon, weil er/sie entzindet ist.

90. Der Pickel ist lastig, weil er/sie schmenzhaft ist.

91. Der Boden ist rutschig, wail er/sie gebohnert wurde.

92. Der Samen keimt schon, weil er/sie gesetzt wurde.

93. Der Wald ist kahl, weil er/sie gerodet wurde.

94. Der Schornstein wird abgerissen, weil er/sie störend ist.

95. Der Reifen ist luftdicht, weil er/sie geflickt wurde.

96. Der Arm ist träge, weil er/sie belastet wurde.

97. Der Wagen kostet viel, weil er/sie neu ist.

98. Der Himmel ist unheimlich, weil en/sie dunkel ist.

99. Der Bailon schwebt oben, weil er/sie leicht ist.

100. Der Whiskey ist angenehm, weil er/sie innen wärmt.

101. Der Rasen ist grinn, weil er/sie beregnet wurde.

102. Der Kort ist biegsam, weill er/sie elastisch ist. 
103. Der Käse ist flüssig, weil ar/sie erwarmt wurde.

104. Dar Tank ist voll, weil er/sie gefullt wirde.

105. Der Scheinwerfer leuchtet wieder, weil ev/sie repariert wurde.

106. Det Schirm ist werkafuflich, weil er/ste genthtn wurde.

107. Der Mantel ist blau, weil arisie gefarbt wurde.

108. Der Mond ist unsichtbar, well extsie verdeckt ist.

109. Der Ring ist unauffindbar, weil er/sie verschwunden ist.

110. Der Computer ist defekt, weil er/sie werwirt ist.

111. Der Zopf ist kurz, weil en/sie abgeschnitten wurde.

112. Der Berg ist anstrengend, weil er/sie hoch ist.

113. Der Gürtell ist eng, weil er/sie fiest ist:

114. Der Balkon ist verboten, weil er/sie brichig ist.

115. Der Wasserhahn tropft, weil er/sie undicht ist.

116. Der Faden ist zerrissen, weill er/sie iberspannt wurde.

117. Der Kiosk ist unbesetzt, weil er/sie geschlossen ist.

118. Der Pudding ist lecker, weil er/sie schokoladig ist.

119. Der Kuchen ist kalorienreich, weil er/sie sahnig ist.

120. Der Teller ist zerbrochen, weil er/sie rumter fiel. 


\section{Appendivit II}

Material for Chapter 2(Experiment 2)

1.

2. Die Blondine hat bald einen Auftritt Die Bluse ist schon, weil sie seiden ist.

b. Die Blondine hat bald einen Auftrit: Die Bluse ist schon, weil sie eltel ist.

c. Der Clown hat bald einen Awftritt. Die Bluse ist schon, weil er seiden ist.

d. Der Clown hat bald einen Auftritt. Die Buse ist schoon, well er eitel ist.

e. Der Clown hat bald einen Auftrit. Die Bluse ist schon, weil sie seiden ist.

f. Der Clown hat bald einen Auftutit. Die Bluse ist schon, well sie eitel ist.

2.

a. Die Zugeunerin ist in einer Backstube. Die Butter ist zerkaffen, weil sie erwarnt wurde.

b. Die Zigeunerin ist in einer Backstube Die Butter ist zerlaufen, weil sie gebacken hat.

c. Der Genglle ist in einer Backstube. Die Butter ist zerlaufen, well er erwarmt wurde.

d. Der Geselle ist in einer Backstube. Die Butter ist zerlaufen, weil er gebacken hat:

e. Der Gesalle ist in einer Backstube. Die Butter ist zerlaufen, weil sie erwarmt wherde.

f. Der Geselle ist in eilner Backstube. Die Butter ist zerlaufen, weil sie gebacken hat.

3.

a. Die Touristin ist auf einer Reise. Die Flasche ist ausgellaufen, weil sie undicht war.

b. Die Touristin ist auf einer Reise. Die Flasche ist ausgelaufen, weil sie unvorsichtig war.

c. Der Onkel ist auf einer Reise. Die lallasche ist ausgelauren, weil er undicht war.

d. Der Onkel ist auf einer Reise. Die flasche ist ausgelaufen, weil er unvorsichtig war:

e. Der Onkel ist auf elner Reise. Die Flasche ist ausgelaufen, weil sie undicht war.

f. Der Onkel ist auf einer Reise. Die Flasche ist ausgelaufen, weil sie unvorsichtig war.

4.

a. Die Ballerina ist in einem Salon. Die Frisur ist furchtbar, weill sie missiungen vst.

b. Die Ballerina ist in einem Salon. Die firisur ist furchtbar, weil sie gewackelt hat.

c. Der Anwalt ist in einem Salon. Die Frisur ist furchtbar, weil er misslungen ist.

d. Der Anwalt ist in einen Salon. Die Frisur ist furchtbar, weil er gewackelt that.

e. Der Anwalt ist in einem Salon. Die Frisur ist furchtbar, weil sie misslungen ist.

f. Der Anwalt ist in einem Salon. Die Fritsur ist furchtbar, weil sie gewackelt hat.

5.

a. Die Gitmetistin ist in eimen Konzert. Die Gutare ist harmonisch, weil sie gestimmt wurde.

b. Die Gitarristin ist in anem Konzert. Die Gitarre ist harmonisch, weil sie musikalisch ist.

c. Ben Gitarrist ist in einem Konzert. Die Gitare ist harmonisch, weil er gestimmt wurde.

d. Der Gitartist ist un eimen Konzert. Die Gitare ist harmonisch, weil er musikalisch ist.

e. Der Gitarrist ist in einem Konzert. Die Gitare ist harmonisch. weil sie gestimmt wurde.

1. Dar Gitartist ist in einem Konzen. Dia Gitarre ist hamonisch, weil sie musikalisch ist.

6.

a. Dia Nonne ist in einen Kirchturm. Die Glocke ist laut, weil sie metallen ist.

b. Die Norne ist in einem Kirchturn. Die Glocke ist laut, weil sie kraftig zieht.

c. Det Pfarter ist in einem Kirchturm. Die Glocke ist laut, weil er metallen ist.

d. Der Pfarrer ist in einem Kirchtum. Die Glocke ist taut, weil er kraftig zieht.

e. Der Pfarrer ist in einem Kirchturm. Die Glocke ist laut, weil sie metallen ist.

f. Dor Pfarrer ist in einem Kirchtum. Die Glocke ist laut, weil ste kraftig zieht.

7 .

a. Die Schwester spivit im Wald. Die Hose ist schnutzig, weil sie schlanmig ist.

b. Die Schwester spielt in Wald. Die Hose ist sehmutzig, weil sie gestürt ist

c. Der Bruder spielt im Wald. Die Hose ist schmutzig, weil er schlammig ist.

d. Der Bruder spielt in Wald. Die Hose ist schmutzig, weil er gesturzt ist.

e. Der Butuder spielt in Wald. Die Hose ist schmutzig, weil sie schlammig ist.

f. Dor Bruder spielt im Wald. Die Hose ist schmatzig, weil sie gestürzt ist. 


\section{8.}

a. Die Frau steht im kalten Schnee. Die Jacke "st warn, weil sio gethtert ist.

b. Die Frat stelit im kalten Schee. Die Jacke ist warm, weil sile fritert schnell.

c. Der Mann steht im kalten Schnee. Die Jacke ist warm, weil er gefuntert ist.

d. Der Mann steht im kalten Sclunee. Die Jacke ist warm, weil er fiert schnell.

e. Der Mann steht im kalten Schnee. Die Jacke ist warm, weil ste gefuttert ist.

f. Der Mann steht im kalten Schnee: Die Jacke ist warm, well sie friert schell.

\section{9.}

a. Die Tochter spielt in einem. Salon. Die Karte ist gezinkt, weil sie markiart ist.

b. Die Tochter spielt in einem Salon. Die Karte ist gezinkt, weit sie haufig schummelt.

c. Der Sohn spielt in einem Salon. Die Karte ist gezinkt, well er markiert ist.

d. Der Sohn spielt in einem Salon. Die Karte ist gazinkt, weil er hafug schummelt.

e. Der Sohn spielt in einem Salon. Die Karte ist gezinkt, weil sie markiert ist.

f. Der Sohn spielt in einem Salon. Die Karte ist gezinkt, weil sie bâfig schummelt.

10.

a. Die Hexe sitzt im dunklen Keller. Die Kerze brent thell, weil sie angezundet wurde.

b. Die Hexe sitzt im dunklen Keller. Die Kerze brennt hell, weil sie sehen möthte.

c. Der Zauberer sitzt im dunklen $\mathbb{K}$ eller. Die Kerze brennt hell, weil er angezundet wurde.

d. Der Zauberer sitzt im dunkten Keller. Die Kerze brennt hell, weil er sehen móchte.

e. Der Zauberer sitzt im dunklen Keller. Die Kerze brennt hell, weil sie angezündet wurde.

f. Der Zauberer sitzt im dunklen Keller. Die Kerze brennt hell, weil sie sehen mochte.

1.1 .

a. Die Diva steht in einem Sclmuckladen. Die Kette ist tener, weil sie anth ist.

b. Díe Diva steht in einem Schmuckladen. Die Kette ist teuer, weil sie reich ist.

c. Der Direktor steht in einen Schmuckladen. Die Kette ist teter, weil er antik ist.

d. Der Direktor steht in einem Schnuckladen. Die Kette ist teuer, well er reich ist.

e. Der Direktor steht in einem Schmuckladen. Die Kette ist teuter, weil sie antik ist

f. Der Direktor steht in einem Schmucklladen. Die Kette ist teuer, weil sie reich ist.

12

a. Die Magd ist in einer Fabrilk. Die Maschine ist laut, weil sie kaputt ist.

b. Die Magd ist in einer Fabrik. Die Maschine ist laut, weil sie ahuungslos ist.

c. Der Pate ist in einer Fabrik. Die Maschine ist laut, weil er kaputt ist.

d. Der Pate ist in einer Fabrik. Die Maschine ist laut, weil er thnungslos ist.

e. Der Pate ist in einer Fabrik. Die Maschine ist laut, weil sie kaputt ist.

1. Der Pate ist in einer Fabrik. Die Maschine ist laut, weil sie ahnungslos ist.

13.

a. Die Plegerin ist in einen Krankenhaus. Die Nase trieft, weil sie verstopht ist,

b. Die Pflegerin ist in einem Krankenhaus. Die Nase trieft, weil sie erkittet ist.

c. Der Patient ist in einem Krankenhaus. Die Nase trieft, weil er verstopft ist.

d. Der Patient ist in einem Krankerhaus. Die Nase trieft, weil er erkthet ist.

e. Der Patient ist in einem Krankenhaus. Die Nase trieft, weil sie verstopft ist.

f. Der Patient ist in einem Krankenhaus. Die Nase trieft, weil sie erkaltet ist.

14.

a. Die Braut ist in einem Laden. Die Schachtel ist deformiert, weill sie zerdrtickt wurde.

b. Die Braut ist in einem Laden. Die Schachtel ist deformiert, weil sie draufgetreten ist.

c. Der Gatte ist in einem Laden. Die Schachtel ist deformien, weil er zerdrückt wurde.

d. Der Gatte ist in einem Laden. Die Schachtel ist deformiert, weil er draufgetreten ist.

e. Der Gatte ist in einem Laden. Die Schachtel ist deformiert, weil sie zerdruckt wurde

f. Der Gatte ist in einem Laden. Die Schachtel ist deformiett, weil sie draufgetreten ist. 
15.

a. Die Kundirigt in encm Schuhladen. Die Socke riech schlecht, wet sile wngewasehen ist.

b. Die Kundin ist in einem Schuhladen. Die Socke riech schlech, weil sie schwitzt sehr.

c. Der Punker ist in emem Schuhladen. Die Socke riecht schlecht, weil er ungewaschten ist.

d. Der Punker ist in winem Schuhladen. Die Socke rilecht schlecht, wail er schwitzt sehr.

e. Der Punker ist in einen Schuhladen Die Socke niecht schilecht, weil sie ungewaschen tst.

f. Dar Puker ist in eunemi Sehulhuden. Die Socke recht schlecht weill sie schwitzt sehr.

16.

a. Die Nichte hat winen Schuh an. Die Sohle ist 100hrig, weil sie durchgelaufen ist;

b. Die Wichte hat einen Schub an. Die Sohle ist Jöehrig, weil sie arm ist.

c. Der Neffe hat einen Schuh an. Die Sohle ist lochrig, weil er durchgelanfen ist.

d. Der Neffe hat einen Schuh an. Die Sohle ist lochrig, weil er arm ist.

e. Der Nefie hat einen Schuh an. Die Solile ist lochrig, weill sie durchgelaufen "st.

f. Dur Meffe hat einen Schuhn. Die Sohle ist Jochrig, weil sie arm ist

17.

a. Die Firstin ist in einem Atelier, Die Skulptur ist anmutig, weil sie filigran ist.

b. Die furstin istist in einem Atelier. Die Skulptur ist annutig, weil sie talentiert ist.

c. Der Kunstler ist in einem Atelier. Die Skulptur ist anmutig, well er filigran ist.

d. Der Kunistler ist in einem Atelier. Die Skulptur ist anmutig; weil er talentiert ist.

e. Der Kunstler ist in einem Atelier. Die Skulptur ist anmutig, weil sie filigran ist.

f. Der Kanstler ist in einem Avelier. Die Skuptur ist anmutig, weill sie talentiert ist.

18.

a. Die Rentnerin lauft selten in Winter. Die Strasse ist glatt, weil sie eisig ist.

b. Die Rentnerin Iauft selten im Winter. Die Strasse ist glat, weil sie gerutscht ist.

c. Der Rentner läuft selten in Winter. Die Strasse ist glatt, weil er eisig ist.

d. Der Rentner lafuft selten im Winter. Die Strasse ist glatt, weil er gerutscht ist.

e. Der Rentner lauft selten um Winter. Die Strasse ist glatt, weil sie eisig ist:

f. Der Rentner läuft selten im Winter. Die Strasse ilst glatt, weil sie gerutscht ist.

19.

a. Die Witwe isst in einem Restaurant. Die Suppe ist scharf, weil sie gewürt ist.

b. Die Witwe isst in einem Restaurant. Die Suppe ist scharf, weil sie bevorzugt Wüze.

c. Der Minister isst in einem Restaurant. Die Suppe ist scharf, weil el gewhzt ist.

d. Der Minster isst in einem Rusaurant. Die Suppe isit schari, weil er bevorzugt Wurze.

e. Der Minister isst in einem Kestaurant. Die Suppe ist scharf, weil sie gew irzt ist.

f. Der Minister isst in einem Restaurant. Die Suppe ist schart, weil sie bevorzugt Wurze.

20.

3. Dia Lehretin ist in ener Schule. Div Tafel ist beschrieben, weil sie genutzt wurde.

b. Die Lehrerin ist in einar Schule. Die Tafel ist beschrieben, weil sile erklart hat.

c. Der Lehrer ist in einer Schule. Die Tafel ist beschrieben, weil er gentzt wurde.

d. Der Lehrer ist in einer Schule. Die Tafel ist beschrieben, weil er erkllart hat.

e. Der Lehrer ist in einer Schule. Die Tafel ist beschrieben, weil sie gentut wurde.

1. Der Lehrer ist in einer Schule. Die Tafel ist beschrieben, weil sie enklärt hat.

21.

a. Die Kaltnerin ist in einer Kithe. Die Tasse ist leer, weil sie abgewaschen ist.

b. Die Kellnerin ist in einer Kinche. Die Tasse ist leer, weil sie getrunken hat.

c. Der Vater ist in einer Kuche. Die Tasse ist leer, weil er abgewaschen ist.

d. Der Vater ist in einer Kuche. Die Tasse ist leer, weil er getrunken hat.

a. Der Vater ist in einer Kuche. Die Tasse ist leer, weil sie abgewaschen ist.

f. Der Vater ist in einer Kithe. Die Tasse ist leer, weil sie getrunken hat. 
22.

a. Die Tante ist in einer "Tischlerei. Die Truhe ist braun, well sie holvern ist.

b. Die Tante ist in einer Tischlereli. Die Truhe ist braun, weill sie Geschmaek hat.

c. Der Kommissar ist in einer Tuschlerei. Die Truhe ist brawn, weil sie holzern ist.

d. Der Kommissar ist in einer Tischlerei. Die Truhe ist bratn, well er Geschmack hat.

e. Der Kommissar ist in einer Tischlerei. Die Truhe ist braun, weil sie holzern ist.

f. Der Kommissar ist in einer Tischlerei. Die Truhe ist braum, weil sie Geschmack hat.

23.

a. Die Sekretärin ist in einer Wohmung. Die Tür ist offen, weil sie kaputt ist.

b. Die Sekretărin ust in einer Wohnung. Die Tür ist offen, weil sie verreisen möchte.

c. Der General ist in einer Wolmung. Die Tür ist offen, weil er kaputt ist.

d. Der General ist in einer Wohnung. Die Thar ist offen, weil er verreisen mobchte.

e. Der General ist in einer Wohnung. Die Tur ist offen, well sie kaputt ist.

f. Der General ist in einer Wohnung. Die Thir ist offen, weil sie verreisen mochte.

24.

a. Die Königin ist in großer Eile. Die Uhr ist genau, weil sie gestellt wurde.

b. Die Königin ist in großer Eile. Die Uhr ist genwu, weil sie punktlich ist.

c. Der Journalist ist in großer Eile. Die Uhr ist genau, weil er gestellt wurde.

d. Der Journalist ist in großer Eile. Die Uhr ist genau, weil er pïnktlich ist.

e. Der Journalist ist in großer Eile. Die Uly ist genau, weil sie gestellt wurde.

f. Der Journalist ist in großer Eile. Die Uhr ist genau, weil sie pinktich ist.

25.

a. Die Sklavin ist in einem Blumengeschäft. Die Vase ist kaputt, weil sie zersprungen ist.

b. Die Sklavin ist in einem Blumengeschaft. Die Vase ist kaputt, weil sie tollpatschig ist.

c. Der Schüler ist in einem Blumengescliaft. Die Vase ist kaputt, weil er zersprungen ist.

d. Der Schüler ist in einem Blumengeschaft: Die Vase ist kaputt, weil sie tollpatschig ist.

e. Der Schiler ist in einem Blumengeschäft. Die Vase ist kaputt, weil er zersprungen ist.

f. Der Schüler ist in einem Blumengesehäft. Die Vase ist kaputt; weil sie tollpatschig ist.

26.

a. Der Abiturient ist in einer Garderobe. Der Anzug ist unbequem, weil er kratzig ist.

b. Der Abiturient ist in einer Garderobe. Der Amzug ist unbequem, weil er nervös ist.

c. Die Dozentin ist in einer Garderobe. Der Anzug ist unbequem, weil sie kratzig ist.

d. Die Dozentin ist in einer Garderobe. Der Anzug ist unbequem, weil sie nervös ist.

e. Die Dozentin ist in einer Garderobe. Der Anzug ist urbequem, weil er kratzig ist.

f. Die Dozentin ist in einer Garderobe. Der Anzug ist unbequem, weil er nervos ist.

27 .

a. Der Jongleur ist in einem Wettkampf. Der Am ist tratge, weil er sclumerzend ist.

b. Der Jongleur ist in einem Wettkampf. Der Arm ist trage, weil er unbesonnen trainiert.

c. Die Sportlerin ist in einem Wettkampt. Der Arm ist trage, weil sie schmerzend ist.

d. Die Sportlerin ist in einem Wettkanmf. Der Arm ist trage, weil sie unbesonnen trainiert.

e. Dije Sportlerin ist in einem Wettkampf. Der Arm ist trage, weil er schmerzend ist.

f. Die Sportlerin ist in einem Wettkampf. Der Arm ist traige, weil er unbesonnen trainiert.

28.

a. Der Fischer ist in einem Verein. Der Bach wird geschutzt, weil er rein ist.

b. Der Fischer ist in einem Verein. Der Bach wird geschutzt, weil er umweltbewusst ist.

c. Die Verkäuferin ist in einem Verein. Der Bach wird geschltzt, weil sie rein ist.

d. Die Verkäuferin ist in einem Verein. Der Bach wird geschutzt, weil sie umweltbewusst ist.

e. Die Verkäuferin ist in einem Verein. Der Bach wird geschutzt, weil er tein ist.

f. Die Verkauferin ist in einem Verein. Der Bach wird geschützt, weil er umwel tbewusst ist. 
29

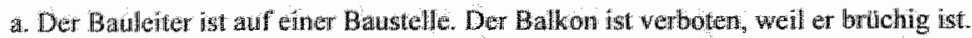

b. Der Bateiter ist auf einet Baustelle. Der Bulkon ist verboten, weil er verantworlich ist.

c. Die Archtektin ist auf einer Baustelle Der Balkon ist verboten, well sie brüchig ist.

1. Die Architektin ist auf einer Bustelle Det Balkon st verboten, weil sie verantwortlich ist.

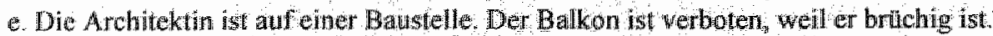

1. Dia Architekth ist auf ene baustelle. Der Bakkon ist verboten, well er verantwortich ist.

30.

a. Der Metger ist in ener Fleischerei. Der Braten ist schmackhaft, weil er gar ist.

b. Des Metzger ist in einer Feischered. Det Braten ist schwnackhaft, weil er talentiert kocht.

c. Die Jungfer ist in einer Feischerei. Der Braten ist schmackhaf, weil sie gar ist.

d. Die Jungter ist in emer Fleseherei. Der Braten ist selimackhaft, weil sie talentient kocht.

c. Die Jungfer ist in einuer Fleischerei. Der Braten ist schmackhaft, well er gar ist.

f. Die Jungfer ist in einer Fleischerei. Der Braten ist schmackhaft, weil er calentien kocht.

31.

a. Der Knecht năht eine Bluse. Der Faden ist zerrissen; weil er porös war.

b. Der Knecht natht eine Bluse. Der Faden ist zertissen, weil er wittend zog.

c. Die Dame naht eine Bluse. Der Faden ist zerrissen, weil sie porös war.

4. Die Dame nath eine Bluse. Der Faden ist zerrissen, weil sie witend zog.

e. Dhe Dame nath eine Bluse. Der Faden ist zerrissen, weil er porös.

f. Die Dame năllt eine Bluse. Der fladen ist zerrissen, weil er kräftig zog.

32.

a. Def Opa ist in einem Heim. Der Garten ist verwildert, weil er ungepflegt ist.

b. Der Opa ist in einem Heim. Der Garten ist verwildert, weil er krank ist.

c. Die Oma ist in einem Heim. Der Garten ist verwildert, weil sie ungepllegt ist.

d. Die Oma ist in enem Heim. Der Garten ist verwildert, weil sie krank ist.

e. Die Ona ist in einem Heim. Der Garten ist verwitdert, weil er ungepflegi ist.

f. Die Oma ist in einem. Hein. Der Garten ist verwildert, weil er krank ist.

3..

a. Der Sanger bleibt heute im Bett. Der Hals ist geschwollen, weil er entzündet ist.

b. Der Sünger bleibt heute im Bett. Der Hals ist geschwollen, weil er krank ist.

c. Die Athletin bleibt heute im Bett. Der Hals ist geschwollen, weil sie entzindet ist.

d. Die Athletin bleibt heute im Bett. Der Hals ist geschwollen, weil sie krank ist.

c. Die Athletin blatbt heute im Bett. Der Mals ist geschwollen, weil er entzindet ist.

C. Die Athletin bleibt heute im Bett. Der Hals ist geschwollen, weil er krank ist.

34.

a. Der Juger ist schon sehr hungrig. Der Herd ist heiß, weil er angeschaltet ist.

b. Der Jager ist schon sehr humgrig, Der Herd ist heiB, weil er kochen inbchte.

c. Dic Polin ist schon setur hungrig. Der Herd ist heib, weil sie angeschaltet ist.

d. Dic Polin ist schon sehr hungrig. Der Herd ist heil, weil er kochen möchte.

4. Die Polin ist sathon sehr hungrig. Der Herd ist heif, weil sie angeschaltet ist.

f. Die Polin ist schon seht hungrig. Der Herd ist heiß, weil ar kochen mochte.

35.

a. Det Professor steht sehr frull aut. Der Kaffee ist stark, weil er anfwecken soll.

b. Der Professor steht sehr frih auf. Der Kaffee ist stark, weil ar aufwachen muss.

c. Die Lelurerin steln selu fruh aut Der Kaffee ist stark, weil sie autwecken soll.

d. Die Lehrerin stetht sehr fouh auf. Der Kaffee ist stark, weil sie aufwachen muss.

c. Die Lehrerin stelt selu fruh auf. Dor Kalfee ist stark, weil er aufwecken solih.

f. Die Lehrerin steht sehr frîh auf. Der Kaffee istl stark, weil er aufwachen muss. 
36 .

a. Der Moderator ist auf der Kellertreppe. Der Keller ist wheimlich, well er dunkel ist.

b. Der Moderator ist auf der Kellettreppe. Der Keller ist unheimlich, meil er angstlich ist.

a. Die Reportierin ist auf der Kellertreppe. Der Keller ist unleimlich, weil sie dunkel ist:

d. Die Reporterin ist auf der Kellertreppe. Der Keller ist unheinhlich, weil sle angstlich ist.

e. Die Reporterin ist auf der Kellertreppe. Der Reller ist umeimlich, weil er dunkel ist.

f. Die Reporterin ust auf der Kellertreppe. Der Keller ist unheimlich, weil er angstlich ist.

37.

a. Der Naffe ist in einem Cafe. Der Kuchen ist kalorienneich, weil er salunig ist.

b. Der Neffe ist in einem Cafe. Der Kuchen ist kalonienreich, weil er zunemmen muss.

c. Die Nichte ist in einem Café. Der Kuchen ist kalorienreich, weil sie salhng ist.

d. Die Nichte ist in einem Cafe. Der Kuchen ist kalorienreich, weil sic zunehmen muss.

e. Die Nichte ist in einem Cale. Der Kuchen ist kalorienreich, weil er sahnig ist.

$f$. Die Nichte ist in einem Café. Der Kuchen ist kalorienreich, weil er zunehmen muss.

38.

a. Der Nart hat bald einen. Auftritt. Der Mund ist rot; weil er durchblutet ust.

b. Der Narr hat bald einer Auftritt. Der Mund ist rot, weil er erkältet ist.

c. Die Kandidatin hat bald einen Auftritt. Der Mund ist rot, weil sie direhblutet ist.

d. Die Kandidatin hat bald einen Auftritt. Der Mund ist rot, weil sie erkathet ist.

e. Die Kandidatin hat bald einen Auftritt. Der Mund ist rot, weil er durchblutet ist.

f. Die Kandidatin hat bald einen Auftritt. Der Mund ist rot, weil er erkiltet ist.

39.

a. Der Koch ist in einem Restaurant. Der Pudding ist lecker, weil er schokoladigg ist.

b. Der Koch ist in einem Restaurant. Der Pudding ist lecker, weil er liebevolli kocht.

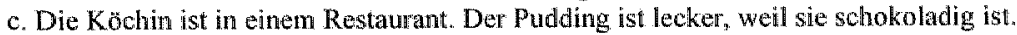

d. Die Kochin ist in einem Restaurant. Der Pudding ist lecker, weil sie liebewoll kocht.

e. Die Köchin ist in einem Restaurant. Der Pudding ist lecker, weil er schokoladig ist.

f. Die Köchin ist in einem Restaurant. Der Pudding ist lecker, weil er liebevoll kocht.

40.

a. Der Hirte ist in einem Garten. Der Rasen ist grün, weil er beregnet wurde.

b. Der Hirte ist in einem Garten. Der Rasen ist grin, weill er fanatisch dungt.

c. Die Forscherin ist in einem Garten. Der Rasen ist grîn, weil sie benegnet wurdle.

d. Die Forscherin ist in einem Garten. Der Rasen ist grön, weil sie fanatisch düngt.

e. Die Forscherin ist in entem Ganten. Der Rasen ist grum, wail er beregnet wutde.

f. Die Forscherin ist in einem Garten. Der Rasen ist grün, weil er fanatisel duagt.

41.

a. Der Gätner ist in einem Beet. Der Samen keint schon, weil er gesetht wutde.

b. Der Gäarther ist in einem Beet. Der Samen keimt schon, weil er sorgsam wassert.

c. Die Sklavin ist in einem Beet. Der Samen keimt schon, weill sie gesetzt wurde.

d. Die Sklavin ist in einem Beet. Der. Samen keimt schon, weil sie sorgsam wassent.

e. Die Sklavin ist in einem Beet. Der Samen kemt schon, weil er gesetzr wurde.

f. Die Sklavin ist in ënem Beet. Der Samen keimt schon, weil en sorgsam wässertt.

42.

a. Der Sänger hat heute einen Umzug. Der Sessel ist schwet, weil er groß ist.

b. Der Sänger hat heute einen Umaug. Der Sessel ist schwer, weill er schwach ist.

c. Die Arztin hat heute einen Umzug. Der Sessell ist schwer, weil sie groß ist.

d. Die Arztin hat heute einen Unzug. Der Sessel ist schwer, weil sie sehwach ist.

e. Die Ärztin hat heute einen Umzug. Der Sessel ist schwer, weil er grob ist.

f. Die Arztin hat heute einen Umzug. Der Sessel ist schwer, weil en schwach ist. 
43.

4. Der Ingenieut ist an ener Tankstelle. Der Tanil ist voll, well er gefull wurde.

b. Der Ingenieur ist an winer Tankstelle. Der Tank ist woll, weil er getankt hat

c. Die Kundin ist an eneir Tankstelle. Der Tank ist voll, weil sie getullt wurde.

d. Die Kundin ist an einet Tankstelle. Der Tank ist woll, well sie getankt hat:

¿. Die Kundin istan einer Tankstelle. Der Tank ist voll, well er gefullit wurde:

f. Die Kundin is an einer Tankstelle, Der Tank ist woll, well er getankat hat:

44.

a. Der Richter ist in einuen Schutuprogham, Der Zaun ist hoch, well er elektrisch ist.

b. Der Richter ist in einem Schutzprogramm. Der Zaun ist hoch, weil er bedroht wird.

c. Die Zeugin int in einem Schutzprogramm, Der Zaun ist hoch, weil sie elektrisch ist.

d. Die Zetugin ist in einem Schutyprogramm. Der Zaun ist hoch, weil sie bedroht wird.

(3) Die Zeugin ist in einem Schurzprogramm. Der Zaun ist hocti, well er elektrisch ist.

f. Die Zeugin ist in einen Schutzprogramm. Der Zaun ist hoch, weill er bedroht wird.

45

a. Der Geselle sucht in einer Werkstatt. Der Zettel ist verschwunden, weil er winzig ist.

b. Der Geselle sucht in einer Werkstatt. Der Zettel ist verschwunden, weil er unordentlich ist.

c. Die Schneiderin sucht in einer Werkstatt: Der Zetrel ist verschwunden, weil er winzig ist.

1. Die Schneiderin sucht in einer Werkstatt. Der Zettel ist verschwunden, weil er unordentlich ist.

e. Die Schneiderin sucht in einer Werkstatt. Der Zettel ist verschwunden, weil er winzig ist.

f. Die Sehneiderin sucht in einer Werkstatt. Der Zettel ist verschwunden, weil er unordentlich ist.

46.

a. Der Siedler ist in einer Kuche. Der Reis ist weich, well er verkocht wurde.

b. Der Stedler ist in einer Kuche. Der Reis ist weich, weil er abgelenkt wurde.

c. Die Chinesin ist in einer Kuche. Der Reis ist weich, weil sie verkocht wurde.

d. Die Chinesin ist in einer Kache. Der Reis ist weich, weil sie abgelenkt wurde.

¿. Die Chinesin ist in einer Kuche. Der Reis ust weich, weil er verkocht wurde.

fi. Die Chinesin ist in einer Kuche. Der Reis ist weich, weil er abgelenkt wurdle.

47 .

4. Der Graf sitzt in einem Cafe. Den Tee ist schlecht, weil er bitter ist.

b. Der Graf sitzt in einem Caft. Der Tee ist schlecht, weil er pingelig ist.

c. Die Baronin sitzt in einem Cafe. Der Tee ist schllecht, weil sie bitter ist.

d. Die Baronin sitzt in einem Cafe, Den Tee ist schlecht, weil sie pingelig ist.

e. Die Baronin sitzt in einem Caft. Der Tee ist schlecht, weil er bitter ist.

1. Die Baronin sittet in einem Cafe. Der Tee ist schlecht, weil er pingelig ist.

48

a. Der Chef ist in einem Hotel. Der Teller ist zerbrochen, weil er zerscheppert ist.

b. Der Chef ist in anem Hotel. Der Teller ist zerbrochen, weil er gestolpert ist.

c. Die Ballerth ist einen Hotel. Der Teller ist zerbrochen, well sie zerscheppent ist.

d. Die Ballerina ist in einem Hotel. Der Teller ist zerbrochen, weil sie gestolpert ist.

e. Die Ballering ist cinem Hotel. Der Tellet ist zerbrochen, well er zerscheppert ist.

f. Die Ballerina ist in elinem Hotel. Der Teller ist zerbrochen, weil er gestolpert ist.

49

Wer Schlosser geht in ein Bett. Der Wecker ist gestellt, weil er klingeh soll.

b. Der Selalosser geht in ein Bett. Der Wecker ist gestellt, weil er autstehen muss.

c. Die Stewardess geht in ein Bett. Der Wecker ist gestellt, weil sie klingeln soll.

d. Die Stewardess gelut in en Bett. Der Wecker ist gestellt, weil sie aufstehen muss.

a. Die Stewardess geht in ein Bett. Der Wecker ist gestellt; weil er khingeln soll.

t. Die Stewardess geht in ein Bett. Der Wecker ist gestellt, weil er aufstehen muss. 
50 .

a. Der Better ist in einer Praxis. Der Zahn ist hasshich, well er gelb, ist.

b. Der Bettler ist in einer Praxis. Der Zahn ist hassilloh, Hoil et putzen vernedet.

c. Die Forscherin ist in eimer Praxis. Der Zahn ist hasshch, well sie gelt ist.

d. Die Forscherin ist in einer Praxis. Der Zahn ist hässlich, weil sie puteen vermeidet.

e. Die Forscherin ist in einer Praxis. Der Zahn ist hăsslich, weil er gelb ist.

1. Die Forscherin ist in einer Praxis. Der Zahn ist hassilch, well putzen wemeidet. 


\section{Appendix IIII}

Material for Chapter 5 athd 6.

\section{Person ans antecedent}

$1 \mathrm{SD}$ Die Arbaterin st eingestellt; wejl sie/er zuwerlassig ist.

LD Die Atbeüterin wird wieder zingestellt und kann werdienen, weil sie/er zuverlãssig ISt.

2 SD Die Athlatin list muskulos, weil sie/er Sport treibt.

LD Die Athlethist richtig fit und ist muskulös, weil sie/er Sport treibt.

3. SD

Die Bullerima schreibt A utogranme, weil siefer berihmt ist.

LD Die Ballerina ist selw gefragt und schreibt Autogramme, weil sieler berühmt ist.

4. $\mathrm{SD}$

Die Baronin ${ }^{3}$ st glocklich, wis sie/er bald heiratet.

LD Dhe Baronin ist sehr nerwós und ist glucklich, weil sie/er bald heiratet:

$5 \mathrm{SD}$

Dike Beamtin ist gestresst, weil sterer Kinder hat.

LD Die Beamtin kann selten entspamnen und ist gestresst, weil sie/er Kinder hat.

$6 \mathrm{SD}$

Die Blondine wollte schwimmen, well sie/er Wasser mag.

L.D

Die Blondine ist gern draußen und wollte schwimmen, weil sie/er Wasser mag.

$7 \mathrm{SD}$

Die Braut ist zittrig, weil sie/er aufgeregt ist.

$8 \quad \mathrm{SD}$

Die Bratt ist heute blass und ist zittrig, weil sie/er aufgeregt ist.

8 LD

Die Chefin ist verzweifelt, weil sie/er betrogen wurde.

$9 \mathrm{SD}$

Die Chefin ist total ruiniert und ist verzweifelt, weil sie/er betrogen wurde.

Die Dame ist traurig, weil stefer einsam ist.

10 SD

Die Dame ist oft deprimient und ist traturig, well siefer einsam ist.

$11 \quad \mathrm{SD}$

Die Diva sagt nie danke und ist stur, weil sieler hochnasig ist.

Die Dozentin ist streng, weil sie/er viel fordert.

Die Dozentin kann selten werzeihen und ist streng, weil sie/er viel fordert.

LD

Die Enkelin ist riedlich, weill sie/er klein ist.

$13 \mathrm{SD}$

Die Enkelin hat rote Haare und ist niedlich, weil sie/er klein ist.

$14 \quad \mathrm{SD}$

Die Etzicherin ist mude, weil sie/er wiel arbeitet.

$14 \quad \mathrm{SD}$

Die Erzieherin geht heute einkaufen und ist nude, weil sie/er viel arbeitet.

$15 \mathrm{SD}$

Die Forscherin ist wissbegierig, weill sie/er interessient ist.

Die Forscherin liest häufig abends und ist wissbegierig, weil sie/er interessiert ist.

$16 \mathrm{SD}$

Die Frau ist beliebt, weil sie/er schön ist

$\mathrm{LD}$

Die Frau wird oft fotografiert und ist beliebt, weil sie/er schön ist.

$17 \mathrm{SD}$

Die freundin backt Kuchen, weil sie/er Geburtstag hat.

Die Freundin gibt eime Peien und backt Kuchen, weil sie/er Geburtstag hat.

L.D

(c)

$18 \mathrm{SD}$

Die Gattin muss spwrsam sein und ist ge

LDD

Die Gottin wird verehrt, weil siefer großzugig ist.

$19 \quad \mathrm{SD}$

Die Gottin ist sehr gut und wird verehrt, weil sie/er grobzagig ist.

Gouvernante katuft Klender, weil siefer eitel ist.

Die Gouvernante verschwendet wiel Geld und kauft Kleider, weil sie/er eitel ist.

Die Greisin wird noch anerkannt und ist weise, weil sie/er alt ist.

21 SD Die Heldin erlebt Abenteuter, weil sie/er mutig ist.

Die Heldin goht aut Reisen und erlebt Abenteuer, weil sie/er mutig ist.

$22 \quad$ SD

Die Hexe ist aufgeregi, weil sie/er Besuch hat.

$23 \mathrm{SD}$

Dic Hexe begint mit kochen und ist aufgeregt, weil sie/er Besuch hat.

Die rim ist spontan, weil siefer verreisen mochte.

Die frim packt schom alles und ist spontan, weil sie/er verreisen möchte.

Die Jungter wird igmoriert, weil sie/er klein ist.

Die Jungfer ist oft traurig und whd ignoriert, weill sieter klein ist.

$25 \mathrm{SD}$ Die Katserin ist ideenreich, weil ste/er kreativ ist.

Die Kaiserin hat alles verändert und is $t$ ideenreich, weil sie/er kreativ ist. 
28 SD Die Kaufrau ist verschuldet; weil siejer maufmerksan war.

LD Die Kaufran muss nun aufgeben und ist verseluldet, weil sieder unatinerksam wai.

29 SD Die Kellnerin ist unsioher, weil sie/et heute beginnt:

LD Die Kellnerin werschüttet die Geträme und ist unsichem, weil siefar lyeute beginnt.

Die Köchin ist verschwitzt, weil sie/er viel arbeitet.

LD Die Kochin nag nach Hause und ist verschwitzt, weil sie/er viel arbeitet.

31 SD Die Königin ist beliebt, weil sieler gut ist.

LD Die Konigun wird sehr verehrt und ist beliebt, weil sierer gut ist.

Die Konkurrentin ist schroft, weil sie/er benachtelligt wurde.

Die Konkurrentin kann ungemütlich sein und ist schroff, weil sie/er benachleilight wirde.

Die Kundin ist modisch, weil sie/er getallen möchte.

Die Kundin kauft neue Sachen und ist modisch, weil siefer gefallen möchte.

Die Lehrerin unterrichtet gut, weil sie/er spontan ist.

Die Lehrerin nag die Arbeit und unterichtet gut, weil sie/er spontan ist. Die Magd hat frei, weil sie/er Urlaub hat.

Die Magd packt schon zusammen und hat frei, weil sie/er Urlaub lkat.

Die Nichte ist allein, weil sie/er of umzog.

Die Nichte geht selten aus und ist allein, well sie/er of umzog.

Die Nonne ist fromm, weil sie/er gläubig ist.

Die Nonne ist sehr bescheiden und ist fromm, weil sie/er glaubig ist.

Die Oma liest gern, weil sie/er Geschichten mag.

LD Die Ona hat viele Bucher und liest gern, weil sie/er Geschichten mag.

Die Pllegerin ist geduldig, weil sie/er warten kann.

Die Pflegerin kann noch hoffen und ist geduldig, weil sieler warten kamn.

Die Polin ist verlobt, weil sie/er verliebt ist.

Die Polin schwebt anf Wolken und ist verlobt, weil siever verliebt ist.

$\mathrm{SD}$ Die Politikerin ist klug, weil sie/er gelernt that.

Die Politikerin wird sehr gefurchtet und ist klug, weil sie/er gelernt hat.

Die Prinzessin war munter; weil sie/ar Schlaf bekam.

Die Prinzessin kam bester Laune und war munter, weil sie/er Schlaf bekam.

Die Professorin ist anwesend, weil sie/er Bespreclinngen hat.

Die Professorin winkt sehr gestresst und ist anwesend, weil sie/er Besprechungen hat.

Die Rentnerin ist wach, weill sie/er Kaffee trank.

Die Rentnerin that schon aufgeräumt und ist wach, weil sie/er Kaffee trank.

Die Reporterin ist verreist, weil sie/er Entspannung sucht.

Die Reporterin hat alles aufgegeben und ist verreist, weil sie/er Entspannung sucht.

Die Schneiderin ist froh, weil sie/e Stofl kautte.

Die Schneiderin beginnt za arbeiten und ist froh, weil sie/er Stoff kathe.

Die Schulerin bat geschwanzt, weil sie/er faul war.

Die Schwedin wird altreten, weil sie/er Klavier spielt.

Die Schwester ist whtend, weil sie/er geargert wurde.

Die Schwester schreit wieder laut und ist wutend, weil sie/er geargent wurde.

Die Sekretärin ist verairgert, weil sie/er Überstunden macht.

Die Sekretarin schimpft lweute laut und ist werărgert, weil sie/er Uberstunden macht.

Die Sklavin ist hungrig, weil sie/er wenig isst.

Die Spielerin ist arm, weil sieler viel verliert.

Die Studentin witd heute befragt und ist nervôs,
Die Tante ist akkurat, weil sie/er Ordnung mag.

Die Tante ist akkurat, weil sie/er Ordnung mag.

Die Tochter ist krank, weil sie/er angesteckt wurde.
Die Tochter kauft schnell Medizin und ist krank, weil siejer angesteckt wurde. 
57 SD Die Touristin ist besorgt, weil sieter krank ist.

LD Die Touristin muss mun ruhen und ist besorgt; weil ste/er krank ist.

5. SD Die Witwe muss wernen, well sicher traurig wt:

LD Die Whwe ist sehr verzweifelt und muss weinertit weil sie/er traurig ist.

59. SD Die Zeugin ist angetlich, weil sie/er bedroht whil.

IDD Die Zeugin muss versteckt werden und ist angstlich, weil sie/er bedroht wird.

60 SD Die Zigenning ist beweglich, weil ste/er vell tarat.

LD Die Zigemern witkt sehr anmutig und ist beweglich, weil sider viel tanzt.

61 SD Der Abiturient ist sehmell, weil er/sie laufen kann.

LD Der Abiturient hat viel trainiert und ist schnel, well er/sie lawfen kann.

62 SD Der Antwalt schlan schlecht, weil er/sie beunrulugt ist.

LD Der Avwalt wurde selbst verklagt und schlaft schlecht, weil er/sie beurnhigt ist.

Der Ara ist lberwbeitet, weil errisie Patienten betreat:

LD Der Arthat keine Zeit und ist oberarbeitet, weil er/sie Patienten betrent:

$64 \mathrm{SD}$ Der Bengel ist langsan, weil eisie verletzt ist.

LD Der Bengel humpelt schon langer und ist langsam, weil er/sie verletzt ist.

65. SD Der Boxer ist stolz, weil er/sie gefeiert wird.

LD Der Boxer hat gestem gewonnen und ist stolz, weil er/sie gefeiert wird.

66 SD Der Bruder ist sportlich, weil er/sie viel trainiert.

LD Der Bnder kanh hoch springen und ist sportlich, weil er/sie viel trainiert.

$67 \mathrm{SD}$

Der Chef war spat, weil er/sie verschlafen hat.

LD Der Chef trank sehwarzen Kaffee und war spät, weil er/sie verschlafen hat.

$68 \mathrm{SD}$ Der Chinurg ist ernst, weil er/sie gerade opertent.

$\mathrm{LD}$ Der Chirurg muss Ruhe haben und ist ernst, weil er/s sie gerade operiert.

Der Clown ist bunt, weil er/sie verkleidet ist.

IDD Der Clown lacht sehr laut tond ist bunt, weil en'sie verkleiclet ist.

70 SD Der Dichter ist poetisch, well er/sie Gedichte schreibt.

LD Der Dichter trinkt sehr gen und ist poetisch, weil er/sie Gedichte schreibt.

$7 \mathrm{SD}$ Der Diener ist anerkannt, weil er/sie fleißig ist.

LD Der Dianer verdient jetzt mehr and ist anerkannt, weil er/sie fleiBig ist.

Der Direktor ist dick, weil en/sie viel isst,

LD Der Direktot mag kaum laufen und ist dick, wieil er/sie viel isst.

Der Englander ist errötet, weil er/s ie schuchtern ist.

Der Englander ist schnell beschämt und ist errotet, weil er/sie schillichtern ist.

Der Faltuer ist gefallurlich, weil er/sie getrunken hat.

Der Fahrer sitzt in Auto und ist gefahrlich, weill er/sie getrunken hat.

Der Fischer ist berwhigt, weil er/sie angeln kann.

Der Fischer hat viel freude und ist beruhigt, weil er/sie angeln kann.

Dor freund ist neugierig, weil er/sie Gertichte hörte.

Der freund schreibt alles auf und ist netgierig, weill er/sie Gerichte hörte.

Der Gatte ist schlafrig, weil er/sie nachts wacht.

Lo Der Gatte ist sehr angespannt und ist schlaftig, weil er/sie nachts wacht.

Der Genetal ist interessiert, weil er/sie Projekte hat.

LD Der General kauft viel ein und ist interessiert, weil er/sie Projekte hat.

Der Gegelle ist undchtsam, weil er/sie mide ist.

LD Der Geselle macht einen Fehller und ist machtsan, weil er/sie made ist.

80 SD Der Hattling ist pfiftug, weil er/sie fuchen konnte.

$\mathrm{ID}$ Der Hattling ist endlich frei tund ist pfiffig, weil er/sie fluchten konnte.

Der Haptling ist kriegerisoh, weil er/sie gewinnen will.

LD Der Haptling greift bald an und ist kriegerisch, well er/sie gewinnen will.

$82 \mathrm{SD}$ Der Herr ist reich, weil en/sie geizig ist.

LD Der Harr ist sehr unbeliebt und ist teich, weil er/sje geizig ist.

Der Hirte ist zuverlässig, weil er/sie Schafe hutet.

$\mathrm{LD}$ Der Hirte ist sehr tierlieb und ist zuverlässig, weil er/sie Schafe hutet.

84 SO Der Ingenieur ist unerfahren, weil er/sie jung ist.

LD Det Ingenieur kann viel anpacken und ist unerfahren, weil er/sie jung ist.

85 SD Der Jager ist ordentlich, weil er/sie suehen hasst.

LD Dor Jäger kam spät zurick und ist ondentlich, weil er/sie suchen hasst:

86 SD Der Jonglieur ist. verschmapft, weil erisie etkältet ist.

LD Der Jonglleur hat einen Husten und ist verschnuptt, weil er/sie erkaltet ist. 
87 SD Der Journalist ist gropzigig, weil er/sie fotos hat

LD Der Journalist ist sehr charmant und ist grofangig, weil er/sie Fowos hat.

88 SD

LD

89 SD

LD

$90 \quad$ SD

LD

$91 \mathrm{SD}$

LD

92 SD

LD

$93 \mathrm{SD}$

LD

$94 \quad \mathrm{SD}$

LD

$95 \quad S D$

LD

96 SD

LD

97 SD

LD

LD

99 SD

LD

$100 \mathrm{SD}$

LD

101 SD

LD

$102 \mathrm{SD}$

LD

$103 \mathrm{SD}$

LD

$104 \mathrm{SD}$

LD

$105 \mathrm{SD}$

LD

$106 \mathrm{SD}$

LD

$107 \mathrm{SD}$

LD

108 SD

LD

$109 \mathrm{SD}$

LD

$110 \mathrm{SD}$

LD

$111 \mathrm{SD}$

LD

$112 \mathrm{SD}$

\section{LD}

113 SD

LD

$114 \mathrm{SD}$

LD

$115 \mathrm{SD}$

LD

$116 \mathrm{SD}$

LD

Der Kamerad ist bescheiden, weil edsile Freunde hat.

Der Kamerad ist sehr belieb und ist bescheiden, weil arsie Freunde hat.

Der Kapitan kennt Lander, weil erisie viel segelt.

Der Kapitän weil sehr wiel und kent Lander, weil er/sie viel segelt:

Der Knecht ist dun, weil er/sie kaum isst.

Der Knecht geht jetzt schlafen und ist dunn, weil er/sie kaum isst:

Der Koch ist dickkopfig, weil er/sie egoistisch ist.

Der Koch ist schnell beleidigt und ist dickkopfig; weil er/sie egoistilisch ist.

Der Kommissar ist efizient, weil er/sie arbeiten muss.

Der Kommissar muss schnell schreiben und ist effizient, weil evisie arbeiten muss:

Dar Laborant ist schlichtern, weil er/sie fremd ist..

Der Laborant arbeitet oft allein und ist schithtern, weil er/sie frend ist.

Der Mann ist geschickt, weil er/sie viel ubt.

Der Mann hat viel Talent und ist geschickt, weil et/sie viel ubt.

Der Minister ist mächtig, weil er/sie reich ist.

Der Minister wird schnell zornig und ist matchtig, weil er/sie reich ist.

Der Monteur ist tatkräftig, weil er/sie bald verreist.

Der Monteur packt schnell zu und ist tatkraftig, weil er/sie bald verreist.

Der Narr ist verrückt, weil en/sie Unsimn treibt.

Der Narr lebt sehr gefîhrlich und ist werrickt, weil er/sie Unsinn treibt.

Der Neffe schleckt Eis, weil er/sie Süßes mag.

Der Neffe kann gut geniefen und schleckt Eis, weil er/sie SuBes mag.

Der Onkel kauft Sußes, weil er/sie Geschenke braucht.

Der Onkel ist sehr beschaftigt und kauft Sußes, weil er/sie Geschenke branclut.

Der Opa ist zerstreut, weil er/sie alt ist.

Der Opa vergisst fast alles und ist zerstreut, weil er/sie alt ist.

Der Patient hustet viel, weil er/sie zuviel raucht:

Der Patient ist noch unvernanftig und hustet whel, weit er/sie zuwiel rancht.

Der Pfarrer tragt schwarz, weil er/sie Gottesdiensit halt.

Der Pfarrer redet sehr viel und tragt schwarz, weil er/sie Gottesdienst halt.

Der Philosoph ist schlau, weil er/sile Pltine hat.

Der Philosoph kann hinterhailtig sein und ist schlau, weill er/sie Platne hat.

Der Pianist ist einsam, weil er/sie viel reist.

Der Pianist hallt wieder weg und ist einuam, weil er/sie viel reist.

Der Pirat ist betrunken, weil er/sie heute feiert.

Der Pirat torkelt heute sehr und ist betrunken, weil er/sie heute feiert.

Der Polizist wirkt autoritar, weil er/sie Unifomen tratgt.

Der Polizist ist sehr distanziert und wirkt autoritur; weil er/ste Uniformen tragt.

Der Prinz ist eitel, weil er/sie hubsch ist.

Der Prinz wirkt oft eingebildew und ist eitel, weil er/sie hubsch ist.

Der Professior ist beschattigt, weil erisie Arbeit hat.

Der Professor kauft schnell ein und ist beschafingt, weil er/sie Arbeit hat.

Der Punker ist auffallig, weil er/sie provozieren will.

Der Punker hat bunte Hare und ist auffallig, weil er/sie prowozieren will.

Der Richter ist verreist, weill er/sie Termine hat.

Der Richter nimmt selten Utlaub und ist verreist, weil er/sie Termine hat.

Der Ritter ist verletzt, well er/sie gekampft hat.

Der Ritter muss vergotgt werden und ist verletzt, weil er/sie getkalmplat hat.

Der Sänger ist bekannt, weil er/sie fantastisch singt.

Der Sanger verdient sehr viel und ist bekannt, weil er/sie fantastischi singt.

Der Schauspieler ist arfolgreich, weil er/sie wheizig ist.

Der Schauspieler artbeiter sehr lange und ist erfolgreich, weil er/sie ehrgeizig ist.

Der Schlosser ist schmutzig, weil er/sie viel arbeitet.

Der Schlosser teparient alles schnell und ist schmutzig. weil er/sie vief arbeitet.

Der Schuler lernt fleißig, weil er/sie Klausuren schrebt.

Der Schuler kann fast alles und lernt fleißig, weil er/sie Klausuren schureibt.

Der Soldat ist kraftig weil er/sie Fritness betreibt.

Der Soldat ist kräftig, weil er/sie litness betreibl.
Der Soldat joggt immer weit und ist kräftig, weil er/sie fitness betreibt. 
117 SD Der Spieler ist enthuschat, weil arsie verloren that.

LD Der spieler whide richtig abgezocktind ist enthuscht, weil er/sie vertoren hat

118 SD Der Spion ist gespame. weil er/sie Auftrige hat.

LD Deir Spion ist sehr aufgeregt und ist gespanint, weil er/sie Aufrage hat:

$119 \mathrm{SD}$ Det Vater ist cungeduldig, well er/sie Ruhe brawth.

LD Der Vater schimptit sehr laut und ist ungeduldig, weil er/sie Ruhe braucht.

$1203 \mathrm{D}$ Der Zauberer ist witzig, weil er/sie Humor hat.

L.D Der Zabuberen hat einen Trick und isit witzig, weil er/sie Humor hat:

\section{Thing as antecedent}

1 SD Die Ampen ist aus, well sic/er defekt ist.

ID Die Armpel muss reparient werden und ist aus, weil sie/er defekt ist.

2. SD Die Bandine ist matschig, weill sie/er uberreif ist.

$\mathrm{LD}$ Die Banane schmeckt sehr seltsam und ist matschig, weil sie/er überreif ist.

$3 \quad \mathrm{D} \quad$ Die Binde ist rotlich, weil sie/er blutig ist.

LD Die Binde nuss gewaschen werden und ist rôtlich, weil sie/er blutig ist.

4 SD Die Blume ist verblaint, weil sie/er vertiocknet ist.

LD Die Blume muss entfernt werden und ist verbluht, weil sie/er vertrocknet ist.

$5 \quad \mathrm{SD} \quad$ Die Bluse ist schön, weil sie/er seiclen ist.

LID Die Blase wird of getragen und ist schon, weil sie/er seiden ist.

$6 \mathrm{SD}$ Die Brille ist durchsichtig, weil siefer gläsem ist.

LD Die Brille wurde endlich geputzt und ist durchsicthig, weil sie/er gläsern ist.

Die Butter ist zerlaufen, weil sie/er erwarmt wurde.

LD Die Butter schmeckt schon ranzig und ist zerlaufen
8 SD Die Couch ist bequem, weil sie/er gepolstert ist.

LD Die Couch ist schon besetzt und ist bequen, well sie/er gepolstert ist.

9 SD Die Decke und ist unerreichbar, wil sie/er hoch ist.

LD Die Decke muss gestrichen werden und ist unerreichbar, weil sie/er hoch ist.

$10 \mathrm{SD}$ Die Fabrik ist zerstört, weil sie/er unmodern ist.

LD Die Fabrik muss restauriert werden und ist zerstört, weill sie/er umodern ist.

Die Flasche ist ausgelaufen, weil sie/er undicht war.

LD Die Flasche ist sehr klebrig und ist ausgelaufen, weil sie/er und cht war.

$12 \mathrm{SD}$ Die Frisur ist furchtbar, weil sie/er misslungen ist.

LD Die Frisur muss werhindert werden und ist furchtbar, weil sie/er misslungen ist.

Die Frucht ist gesund, weil sie/er Vitamine hat.

LD Die Frucht wird oft empfohlen und ist gesund, weil sie/er Vitamine hat.

Die Gabel hat Gewicht; weil siefer metalien ist.

LD Die Gabet ist schon gefornt and hat Gewicht, weil sie/er metallen ist.

Die Galerie ist interessant, weil sie/er Werke zeigt.

LDD Die Galerie wird viel besucly und ist interessant, weil sieler Werke zeigt.

Die Gitarre ist hamonisch, weil sie/er gestimmt wurde.

LD Die Gitarre klingt sehr schör und ist harmonisch, weil sie/er gestimmt wurde.

Die Gllocke ist laut, weil sie/er grob ist.

L.D Die Gllocke ist seht schwer und ist laut, weil siefer grofs ist.

$185 \mathrm{D}$ Die Halle ist riesig, weil sie/er vergroflert wurde.

LD Die Halle wird of genutht und ist riesig, weil sie/er vergroBert wurde.

Die Heizung ist heili, weil sieler an ist.

LD Die Hoizung warmt jetzt endlich und ist heill, weil siefer an ist.

20 SD Die Hose ist schmutzig, weil sie/ar schlanunig ist.

LD Die Hose muss gewaschen werden und ist schmutzig, weil sie/er schlammig ist.

$21 \mathrm{SD}$ Die Jacke ist warm, weil sie/er gefuttert ist.

LD Die dacke ist sehr kuschelig und ist wam, weil sie/er gefuittert ist.

22 SD Die Karte ist gezinkt, weil sie/er markiert wurde.

LD Die Karte wird oft versteckt und ist gezinkt, weil siefer markiert wurde.

$23 \mathrm{SD}$ Die Kathedrale ist kalt, weil sie/er ungeheizt ist.

LD Die Kathedrate ist riesig grol und ist kalt, weil sie/er ungeheizt ist.

24 SD Die Kerze bretunt hell, weil sie/er angezundet wurde.

LD Die Kerze ist schon klein und brennt hell, weil sie/er angezilndet wurde.

25 SD Die Kette ist tener, weil sie/er antik ist.

LD Die Kette wird jetzt ausgestelt und ist teuer, weil siefer antik ist. 
26. SD Die Klinke ist lila, well sie/er lackert wurde.

LD Die Klinke ist nun klebrig wnd ist lila, weil sie/er licklert whrde.

27 SD Die Kneipe ist woll, weil siefer bellebt ist.

LD Die Kneipe ist gut besucht und ist vol, weil siefer bellebt ist. Die Krawatte ist schick, weil sie/er modern ist.

Die Krawatte wird oft getragen und ist schick, weil sie/er modern ist.

LD Die Krone ist sehr bekannt und funkelt hell, well siever golden ist.

Die Limonade ist kalt, weil sie/er gekuhlt wurde.

Die Limonade ist schön erfrischend und ist kalt, weil steder gekthlt wurde.

Die Maschine ist latit, weil sieler wackelig steht.

Die Maschine springt ein bisschen und ist laut, weil sie/er wackelig steht.

Die Nase ist sehr verstopt und trieft stark, weil sie/er verschnupti isi.

LD Die Perle wird gut bewacht und ist wertvoll, weil sie/er selten ist.

Die Rakete fliegt los, weil sie/er gestartet wurde.

LD Die Rakete hat laut geknall und fliegt los, weil sie/er gestartet wurde.

Die Salbe ist lindernd, weil sie/er Schmerzen stillt.

LD Die Salbe ist sehr hilffeich und ist lindernd, weil ste/er Sclunerzen stillt.

Die Schachtel ist deformiert, weil sie/er zerdrückt wurde.

LD Die Schachtel war sehr schön und ist deformiert, weil sie/erzerdrtickt wurde.

$37 \mathrm{SD} \quad$ Die Scheibe ist Klar, weil sie/er geputzt wurde.

LD Die Scheibe ist richtig blank und ist klar, weil sie/er geputzt wuide.

Die Schere ist scharf, weill sie/er geschliffen wurde.

LD Die Schere ist wieder spitz und ist schanf, weil sie/er geschliffen wurde.

Die Schiene wird gewechselt, weil siejer porös ist.

Die Schene ist sehr gefahrlich tond wird gewechselt, weil sie/er porbs ist.

Die Schule ist geschlossen, weil siefer penoviert wird.

Die Schulle ist sehu alt und ist geschlossen, weil sie/er renoviert wird.

Die Schüssel wird weggeworfen, weil sieler Risse hat.

Die Schüsel ist nun aussortient und wird weggeworfen, weil sie/er Risse hat.

Die Skulptur ist anmutig, weil sie/er filighan ist.

Die Skulptur ist fein gearbeitet und ist anmutig, weil sie/er filigran ist.

Die Socke riecht schlecht, weil sie/er ungewaschen ist

Die Socke ist etwas muffig und riecht schlecht, weil sie/er ungewaschen ist.

Die Sohle ist löchrig, weil sie/er durchgelauten ist.

Dite Sohle ist sehr porös und ist löchrig, weil siefer durchgelaufen ist.

Die Sonne blendet stark, weill sie/er grell ist.

Die Sonne scheint heute kraftig und blendet stark. weil ste/er grell ist.

Die Stange ist schwer, weil sie/er bleiern ist.

Die Stange wird kaum getragen und ist sclwwer, weil sie/er blefleri ist.

Die Straße ist glatt: weil siever vereist ist.

Die Strabe ist sehr gefalntich und ist glatt, weil sieler vereist ist.

Die Stufe ist solnwierig, weil sieler hoch ist.

Die Stufe wird of gemieden und ist schwierig, weil sie/er hoch ist.

Die Suppe ist scharf, weil sie/er gewurat wurde.

Die Suppe schmeckt sehr gut und ist schart, weil sie/er gewtrat wurde.

Die Tablette ist angestind, weil sie/er giftig ist.
Die Tablette nuss gemieden werden und ist ungesund, weil sie/er giftig tost.

Die Tafel ist beschrieben, weil sieler genutz wurde.

Die Tafel muss geputzt werden und ist beschrieben, weil sie/men genuitzt wurde.

Die Tasche ist praktisch, weil sic/en ubersichtich ist.
Die Tasche ist sehr groß und ist praktisch, weil sie/er ubersichtlich ist.

Die Tasse ist leer, weil sie/er ausgetrunken wurde.

Die Tinte ist verschmiert, weil sie/er feucht war.

Die Tinte ist verschmiert, well ste/er feucht war.
Die Tinte ist schón blat the ist verschmiert, weil sie/er feucht war. 
56 SD Die Truhe lsil brawn, wen sieler holzern ist.

LD Dive Truhe sist sehr schon und ist bratn, weil sie/er holzem ist.

$\$ 7$ SD Die Tor ist offen, weil sie/er kaput ist.

LD Dhe Tor russ reparier werden und ist offen, well sieler kaputt ist

$58 \mathrm{SD}$

LD Die Uht geht ganz prazise und ist genau, weil sie/er gestellt wurde.

$59 \mathrm{SD}$ Die Vase ist kaput,, weil siever gefallen ist.

LD Die Vase muss entsorgt werden und ist kaputt, weil sireler gefallen ist.

LD Die Wunde ist leider entzundet und ist schmerzend, weil sie/er tief ist.

61 SD Der Anzug ist unbequem, weil er/sie steif ist.

LD Der Anzug kratat gehr stark tund ist unbequem, weil er/sie steif ist.

$62 \mathrm{SD}$

Der Apfell ist stin, weil er/sie reif ist.

$63 \quad \mathrm{SD}$

Der Aplel ist schön saftig und ist suB, weil er/sie relf ist.

LD

Der Am ist trige, well er/sie belastet wurde.

Der Arm ist heute kraftlos und ist trage, weil er/sie belastet wurde.

Der Bach ist geschutzt, weil er/sie rein ist.

er Bach muss behutet werden und ist geschutzt, weil er/sie rein ist.

Dei" Balkon ist verboten, weil er/sile bribchig ist.

Der Balkon muss gestuter werden und ist verboten, weil er/sie brïchig ist.

Der Ballon schwebt oben, weil er/sie leicht ist.

Der Ballon schaukelt ein wenig und schwebt oben, weil er/sie leicht ist.

Der Berg ist anstrengend, weil er/sie hoch ist.

Der Berg ist oben eisig und ist anstrengend, weil er/sie hoch ist.

Der Boden ist rutschig, weil er/sie gebohnert wurde.

Der Boden ist noch feucht und ist rutschig, weil er/sie gebohnert wurde.

Der" Braten ist schmackhaft, weil er/sie gar ist.

Dar Braten ist knusprig bram und ist schmackhaft, weil er/sie gar ist.

Del Computer ist defekt, weil er/sie vervirt ist.

Der Computer muss ausgesondert werden und ist defekt, weil er/sie vervirt ist.

Der Faden ist zerrissen, weil er/sie uberspannt wurde.

Der Faden wurde dünn gesponnen und ist zerrissen, weil er/sie überspannt wurde.

72 SD Der Fernseher ist gut, weil er/sie neu ist.

Der Fernseher ist technisch aktuell und ist gut, weil ar/sie neu ist.

Der Ftller ist pink, weil er/sie auffallen soll.

Der Fuller schreibt sehr gut und ist pink, weil er/sie auffallen soll.

Der Garten ist verwildert, weil er/sie unbewohnt ist.

Der Garten soll verkauft werden und ist verwildert, weil er/sie unbewohnt ist.

Der Gurtell ist eng, weil er/sie fest ist.

Der Gurtel sitzt sehr stramm und ist eng, weil er/sie fest ist.

Der Hals ist geschwollen, weil er/sie entzind et ist.

Der Hals schmerzt sehr stark und ist geschwollen, weil er/sie entzlindet ist.

Der Herd ist heifl, weil ar/sie an ist.

Den Herd ist sehr schmuzig und ist heib, weil er/sie an ist.

Der Kase wird flussig, weil ar/sie enwarmt wurde.

Der Käse ist schön geschmeidig und wird hüssig, weil er/sie erwärmt wurde.

Der Kaflee ist stark, weil er/sie aufwecken soll.

Der Kaffee ist endlich fertig und ist stark, weil er/sie aufwecken soll.

Der Keller ist unhemlich, weil er/sie dunkel ist.

Der Keller ist sehr kalt und ist unheimlich, weil er/sie dunkel ist.

Der Kiosk ist unbesetz, weil er/sie geschlossen ist.

Der Kiosk muss schnell weg und ist unbesetzt, weil er/sic geschlossen ist.

Der Knopf ist ab, weil ersie lose war.

Der Knopf ist sehr klein und ist ab, weil erisie lose war.

Der Korb ist biegsam, weil er/sie elastisch ist.

Der Korb wird oft genutzt und ist biegsam. weil er/sie elastisch ist.

Der Kuchen ist kalorienneich, weil er/sie sahnig ist 
86. SD Der Mond ist unsichtbar, well er/sie vendeckt ist.

LD Der Mond ist heute verschwunden und ist unsichtbar, weil er/sie verdeckt ist.

87 SD Der Mund ist rot, weill er/sie durchblutet ist.

LD Der Mund kann fantastisch lachen und ist rot, weil er/sie durdublutet ist.

88 SD Der Nagel ist rostig, weil er/sie nass war.

LD Der Nagel muss gewechselt werden und ist rostig, weil er/sie mass war.

89 SD Der Pickel ist lastig, weil er/sie schmerzhaft ist:

LD Der Pickell muss behandelt werden und ist likstig; weil er/sie schmerzhaft ist.

$90 \mathrm{SD}$ Der Pudding ist lecker, weil er/sie schokoladig ist.

LD Der Pudding muss gekulhit werden und ist lecker, weil er/sie schokoladig ist.

$91 \mathrm{SD}$ Der Rasen ist grùn, weil an/sie beregnet wurde.

LD Der Rasen wurde frisch gemaht und ist grïn, weil er/sie beregnet wurde.

$92 \mathrm{SD}$

Der Relfen ist Jufticht, weil er/sie geflickt wurde.

LD Der Reifen kann genutzt werden und ist luftdicht. weil er/sie geflickt wurde.

$93 \mathrm{SD}$

Der Ring ist unauffindbar, weil ar/sie verschwunden ist.

LD Der Ring ist seh" wertvoll und ist unauffindbar, weil er/sie verschwunden ist.

$94 \mathrm{SD}$

Der Reis ist weich, weil er/sie verkocht wurde.

LD Der Reis ist leider klebrig und ist weich, weil er/sie verkocht wurde.

$95 \mathrm{SD}$

Der Rucksack ist schwer, weil er/sie gepackt ist.

LD Der Rucksack wurde schnell getlickt und ist schwer, weil er/sie gepackt ist.

$96 \mathrm{SD}$

Der Saft ist lecken, weil er/sie frisch ist.

LD Dar Saft kostet sehr viel und ist lecker, weil er/sie frisch ist.

97 SD

LD

Der Samen keint schon, weil en'sie gesetat wurde.

Der Samen ist noch klein und keimt schon, weil er/sie gesetzt wurde.

98 SD

L.D

Der Scheinwerfer leuchtet wieder, weil er/sie repariert wurde.

$99 \mathrm{SD}$

Der Scheinwerfer wird wieder genutzt und leuchtet wieder, weil ar/sie reparient wurde.

LD

Der Schirm ist verkäuflich, weil er/sie genäht wurde.

$100 \mathrm{SD}$

Der Schirm ist wieder repariert und ist verkänflich, weil er/sie genatht wurde.

SD Der Schornstein wird abgerissen, weil er/sie störend ist.

$101 \mathrm{SD}$

Der Schornstein ist richtig hässlich und wird abgerissen, weil er/sie storrend ist.

Der Schrank ist morsch, weil er/sie Wiirner hat.

102 SD

Der Schrank quietscht immer laut und ist morsch, weil er/sie Wurmer hat.

Der Schuh ist kaputt, weil er/sie alt ist.

103 SD

$\mathrm{LD}$

Der Schuh ist nun löchrig und ist kaputt, weil erisie alt ist.

Der Sessel ist schwer, weil er/sie grol ist.

$104 \mathrm{SD}$

Der Sessel ist seh" bequem und ist schwer, weil er/sie groß ist.

$1 . \mathrm{D}$

Der Tag ist mild, weil erisie sonnig ist.

$105 \mathrm{SD}$

L.D

Der Tag karn nun beginnen and ist mild, weil er/sie sonnig ist.

Der Tank ist voll, weil er/siegefullt wurde.

$106 \mathrm{SD}$

Der Tank rostet ein wenig und ist voll, weil er/sie gefoull wurde.

LD

Der Tee ist schlecht, weil er/sie bitter ist.

$107 \mathrm{SD}$

$\mathrm{LD}$

Der Tee wird schnell kalt und ist schlecht, weil er/sie bitter ist.

Der Teller ist zerbrochen, weil er/sie runter fiel.

1.08. $\mathrm{SD}$

Der Teller ist schön bemalt und ist zerbrochen, weil er/sie runter fiel.

L.D

Der Topf ist unbrauchbar, weil er/sie Locher that.

$109 \mathrm{SD}$

Der Topf ist total leer und ist unbrauchbar, weil en/sie Locher hat.

Der Tunnel ist dunkel, weil er/sie lang ist.

LiD

Der Tunnel wird kaum belenchtet und ist dunkel, weil er/sie lang ist.

$110 \mathrm{SD}$

Der Wagen kostet wiel, weul ertsie neu ist.

$\mathrm{LD}$

$111 \mathrm{SD}$

Der Wagen ist sehr schnell und kostet viel, weil en/sie neu ist.

Der Wald ist kahl, weil er/sie gerodet wurde.

LD

112 SD

Der Wald ist praktisich tot und ist kahl, weil er/sie gerodet wurde.

Der Wasserhahn tropft laut, weil er/sie undicht ist.

113. $\mathrm{SD}$

Der Wasserhahn muss repartiert werden und tropf laut, weil er/ste undicht ist.

Der Wecker ist gestellt, weil en/sie klingeln soll.

Der Wecker tickt sehr laut und ist gestellt, weil er/sie klingeln soll

Der Wein schmeckt gut, weil er/sie trocken ist.

$\| \Perp 5 \quad \mathrm{SD}$

Der Wein wird gern getrunken und schneckt gut, weil er/sie trocken ist.

Der Whiskey ist angenehm, weil er/sie innen warmt.

Der Whiskey wird oft gekauft und ist angenehm, weil er/sie innen warmt. 
116 sD Der Zahn ist hasshich, weil er/sie gelbist.

$\mathrm{LD}$ Der Zahn muss gezogen werden und ist hadsslich, weil er/sie gelb ist.

$117 \mathrm{SD}$ Dêr Zatum ist hoch, weil er/sie schitzen soll.

LD Der Zaun wurde exira werstarkt und ist hoch, well er/sie schuticen soll.

118 SD Derzentel st verschwunden, well er/ste winzig war.

Lo Der Zettel st sehr wichtg und ist verschwtnden, weil er/sie winzig war.

$119 \mathrm{SO}$ Der Zopf ist kurz, weil er/sie abgescllmitten wurde.

LD Der 7opf ist schon blond und ust kurz, weil er/sie abgeschnitten wurde.

$120 \mathrm{SD}$ Der Zug ist spat, weil en/sie angehalten wurde.

LD Der Zug ist sehr voll und ist spat, weil er/sie angehalten wurde. 


\section{Acknowledgments}

For their ongoing support and valuable advices during my $\mathrm{PhD}$ period I want to thank my supervisors and advisors Bernadette Jansma, Rainer Goebel and Thomas Münte. I greatly appreciate their discussions and valuable criticism. It was a personal pleasure and a gain for my scientific development to work with such inspiring and supporting personalities.

More personally I would like to thank Bernadette for her friendship. Even though you had a thousand-and-one other tings to do you always found time for me: to listen, to discuss, keeping me motivated by your own enthusiasm, or just to have a coffee together. 1 enjoyed working with you and I am grateful that you gave me the freedom to do the work my way.

I thank all the colleagues and coworkers who contributed to my work and the atmosphere at the institutes in Maastricht and Magdeburg. Special thanks go to Mart Bles (for reading the thesis and discussions), Henk Jansma (for being a friend, introducing me to BrainVoyager, preparing the Dutch translation of the summary), Annemie Jeukens (who had the answer to any organizational question, who 1 kept busy with travel applications - and always a smile), Monique Lamers (for discussions and comments on the first chapter), Sonja Huntgeburth (for being a friend), Eric van Loesbroek (for being a nice office mate), Steffi Pfeifer (for her help with subjects), Bettina Sorger (remember Majellastraat?), Nienke van Attveld, Amanda Kaas, Milene Bonte, Ingrid Christoffels, Niko Kriegeskorte and Alard Robroek from Maastricht (for the discussions and the nights out in Maastricht). In Magdeburg I want to thank Michael Scholz, Claus Tempelmann, Boukje Habets, Nina Noennig, Stefan Knape, Ariel Schoenfeld, Daniel Wiswede (for being nice colleagues, discussions acquiring data and help with data analysis). In addition I thank all the subjects who took pant in my experiments.

Henk and Mart volunteered to be my paranimfen and I am already grateful for their help. Privately I would like to thank the Jansma family, Ella, Linus, Bernadette and Henk, as they gave me a pleasant home in Maastricht. I would like to thank my whole family for letting me become the person I am today and for their ongoing support in all situations. Last, but important, I want to thank Malte. 


\section{Curriculum Vitae}

1976

$1996-2001$

1997-1998

1998-2001

2001

2001-2004

2004 - present
Born in Burg, Germany

Study of Psychology, Otto-von-Guericke University, Magdeburg

Student Research Assistant, Department of Social Psychology, Otto-vonGuericke University, Magdeburg

Student Research Assistant, Department of Neuropsychology, Otto-vonGuericke University; Magdeburg.

M.A. Psychology. Thesis: Amyotrophic Lateral Sclerosis (ALS) and possible extra-motor effects investigated by ERP and neuropsychological tests Supervisors: Prof. T. F. Münte, Prof. H.J. Heinze

PhD student, Department of Neurocognition, Faculty of Psychology, Maastricht University. Semantics, syntax and working memory during pronoun resolution investigated by temporal and spatial neuroimaging. Supervisors: R. Goebel, B. M. Jansma.

Researcher, Department of Neurocognition, Faculty of Psychology Maastricht University 
